\title{
THE PATH TO HIGH Q-FACTORS IN SUPERCONDUCTING ACCELERATING CAVITIES: FLUX EXPULSION AND SURFACE RESISTANCE OPTIMIZATION
}

\section{BY}

\section{MARTINA MARTINELLO}

Submitted in partial fulfillment of the requirements for the degree of Doctor of Philosophy in Physics in the Graduate College of the Illinois Institute of Technology

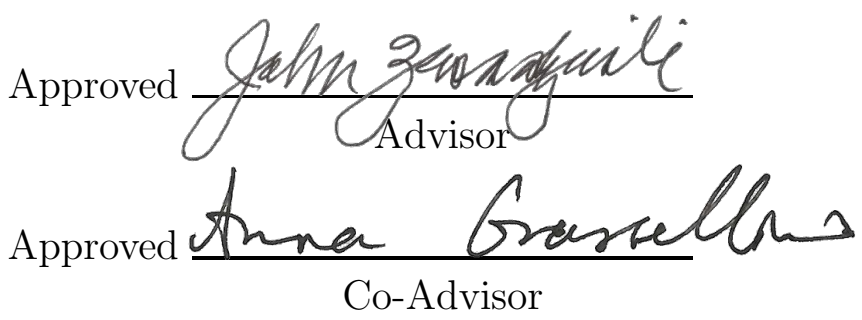

Chicago, Illinois

December 2016 
(C) Copyright by

MARTINA MARTINELLO

December 2016 


\section{ACKNOWLEDGMENT}

Firstly, I would like to express my sincere gratitude to my supervisor, Dr. Anna Grassellino, and my advisor, Prof. John Zasadzinski, because they both gave me the possibility to undertake the path toward this Ph.D degree. During my Ph.D experience I had the possibility to work with state-of-the-art facilities, in an exiting and stimulating environment which creates the perfect conditions to learn fast and with interest, not only about my specific area of research, but about the whole fascinating field of particle accelerators. Without their support this would not have been possible.

I would like to acknowledge Dr. Anna Grassellino also for her guidance, encouragements and suggestions during my Ph.D studies. Her guidance helped me in all the aspects of my research, and inspired me to conduct my work with passion and dedication. I would like to thank her for allowing me to grow as a research scientist and to have tremendously contributed to my career development.

I am very thankful to all the people from the Fermilab SRF group: Dr. Anna Grassellino, Dr. Alexander Romanenko, Dr. Olexandr Melnychuck, Dr. Dmitri Sergatskov, Dr. Sam Posen, Dr. Yulia Trenikhina, Dr. Sebastian Aderhold. A special thanks to Dr. Alex Melnychuck for his help on the RF test organizations and measurements. A special thanks also to Dr. Dmitri Sergatskov for his help on the cavity instrumentation, RF measurements and paper writings. A deep and sincere gratitude to Dr. Alex Romanenko for his help and guidance in different aspects of my Ph.D research, for the innumerable discussions that have contributed immensely to the evolution of my ideas on the Ph.D research.

I would like to acknowledge Prof. Hasan Padamsee for supporting my research work at Fermilab and for the useful discussions during the SRF meetings. I would like to acknowledge also the Fermilab SRF Department head, Dr. Vyacheslav Yakovlev, 
and the Fermilab Technical Division head, Dr. Sergey Belomestnykh, for supporting my research work at Fermilab.

I would like to acknowledge Prof. John Zasadzinski also for always being available to discuss the research work and for the precious comments and suggestions that he gave me.

I am thankful to Prof. Carlo Segre because of his huge help at the beginning of the Ph.D program.

In addition, I would like to acknowledge all my family, especially my mom Trinidad, which gave me the support and strength to begin this overseas experience.

Thanks also go to all the new friends that made me feels good and enjoy the time during these years. A special thanks to Antonio who is the first person I met at university and become from that moment on a very good friend, to Nicolo' for the good time spent together in Chicago, to Paolo for being one of those friends I can rely on.

Finally, I wish to express my unqualified thanks to my boyfriend and colleague Mattia Checchin to have shared with me this experience and to always strive towards my goal. I would not have been able to complete this thesis work without his love and encouragement. 


\section{TABLE OF CONTENTS}

Page

ACKNOWLEDGEMENT . . . . . . . . . . . . . . . . . . . . . iii

LIST OF TABLES . . . . . . . . . . . . . . . . . . . . . . v vii

LIST OF FIGURES . . . . . . . . . . . . . . . . . . . . . . . . . xiv

LIST OF SYMBOLS . . . . . . . . . . . . . . . . . . . . . . . . $\mathrm{xv}$

ABSTRACT ......................... $x i x$

CHAPTER

1. INTRODUCTION ................... . . . 1

1.1. The importance of high Q-factors for particle accelerators . 1

1.2. Thesis goals and organization . . . . . . . . . . . 3

2. RADIO-FREQUENCY ACCELERATING CAVITIES . . . . . 7

2.1. Accelerating cavities . . . . . . . . . . . . . . . . . . . 7

2.2. Figure of merits . . . . . . . . . . . . . . . . . . . . . 11

2.3. Cavities classification . . . . . . . . . . . . . . . 14

2.4. Advantages of superconductivity . . . . . . . . . . . 15

3. INTRODUCTION TO SUPERCONDUCTIVITY . . . . . . . 19

3.1. Overview .... . . . . . . . . . . . . . . . . . . 19

3.2. Two fluid model . . . . . . . . . . . . . . . . . . . . 21

3.3. From local to non-local electrodynamics . . . . . . . . . . 24

3.4. The Ginsburg and Landau theory . . . . . . . . . . . . . 26

3.5. The BCS theory . . . . . . . . . . . . . . . . . 29

4. RF MEASUREMENTS AND CAVITY PROCESSING . . . . . 38

4.1. Power balance in a resonator . . . . . . . . . . . . . . . 38

4.2. RF measurements . . . . . . . . . . . . . . . . . . . . 42

4.3. Q-factor versus accelerating field curves . . . . . . . . . . 48

4.4. Niobium cavities fabrication and preparation . . . . . . . 50

4.5. The nitrogen-doping treatment . . . . . . . . . . . . . . 55

4.6. Surface treatments of the studied cavities . . . . . . . . . 58

5. MAGNETIC FLUX EXPULSION IN HORIZONTALLY COOLED CAVITIES . . . . . . . . . . . . . . . . . 62

5.1. Introduction . . . . . . . . . . . . . . . . 62 
5.2. Experimental set-up _. . . . . . . . . . . . . . . . 64

5.3. Horizontal cooldown data analysis . . . . . . . . . . . . 66

5.4. Physics behind the flux hole scenario . . . . . . . . . . 77

5.5. Conclusions . . . . . . . . . . . . . . . . . . . . 78

6. COOLDOWN DYNAMICS: FAST VS SLOW . . . . . . 80

6.1. Introduction . . . . . . . . . . . . 80

6.2. Thermodynamic force acting on a vortex . . . . . . . 82

6.3. Statistical model of the flux expulsion . . . . . . . . . . . 86

6.4. Cooldown dynamics recorded via T-map . . . . . . . . . . 103

6.5. Conclusions . . . . . . . . . . . . . . . . . 116

7. SURFACE RESISTANCE DEPENDENCE ON THE MEAN FREE

$\mathrm{PATH} \ldots \ldots \ldots \ldots$

7.1. Introduction . . . . . . . . . . . . . . . . . 119

7.2. Experimental procedure . . . . . . . . . . . . . . 121

7.3. Mean free path calculation . . . . . . . . . . . . . 124

7.4. Trapped flux sensitivity . . . . . . . . . . . . . . . . . . . 129

7.5. Theoretical explanations of the dissipation due to trap flux . 131

7.6. BCS surface resistance analysis . . . . . . . . . . . . . 141

7.7. The best surface treatment for SRF cavities . . . . . . . . 144

7.8. Conclusions . . . . . . . . . . . . . . . . 147

8. CONCLUSIONS . . . . . . . . . . . . . . 150

BIBLIOGRAPHY . . . . . . . . . . . . . . . . . 155 


\section{LIST OF TABLES}

Table

Page

4.1 Summary of the surface treatments of the cavities studied in this thesis work. . . . . . . . . . . . . . . . . 59

5.1 Cooldowns summary and associated measured residual resistance. . $\quad 66$

6.1 Summary of the parameters of the Gaussian probability density functions used to describe flux expulsion data of AES011 and CBMM. .

6.2 Summary of the parameters of the double peaked probability density function used to describe flux expulsion data of the cavities ACC002, CBMM, AES017 and AES017b. . . . . . . . . . . . . . . .

7.1 Summary of the cavity surface treatments with the estimated values of mean free path, trapped flux sensitivity at $5 \mathrm{MV} / \mathrm{m}$ and $2 \mathrm{~K}$ BCS surface resistance at $16 \mathrm{MV} / \mathrm{m}$ (unless specified otherwise). . . . . 


\section{LIST OF FIGURES}

Figure

Page

2.1 Sketch of a pill-box cavity with radius $a$ and length $h . \quad$. . . . . 8

2.2 Axial (top) and polar (bottom) plots of the field $E_{\mathrm{z}}$ (a) and $B_{\phi}$ that resonates in the $\mathrm{TM}_{010}$ mode inside a pill-box. Both the fields are normalized for the electric field amplitude $E_{0}$. . . . . . . . . . .

2.3 Electric a) and magnetic b) field density in an elliptical $1.3 \mathrm{GHz}$ Tesla type cavity. The dark red region indicates the highest field density, while the dark blue region indicates the lowest. . . . . . .

2.4 (a) Sketch of a single-cell elliptical cavity, electric and magnetic field resonates in the cavity cell, while the beam tube is needed for the passage of the beam. (b) surface electric and magnetic field distribution as a function of the distance along the beam axis, from the center of the cavity. The peak surface magnetic field is very close to the cavity equator, while the peak surface electric field is close to the cavity irises. . . . . . . . . . . . . . . . . . . .

3.1 Critical magnetic field $H_{\mathrm{c}}$ as a function of the temperature $T$. For $H<H_{\mathrm{c}}$ the material is in the superconducting (SC) state, while for $H>H_{\mathrm{c}}$ it is in the normal-conducting (NC) state. . . . . . . . .

3.2 Variation of the penetration depth with the mean free path [1]. . .

3.3 (a) Lower $\left(H_{\mathrm{c} 1}\right)$ and upper $\left(H_{\mathrm{c} 2}\right)$ critical magnetic fields as a function of the temperature for a type II superconductor. For $H<H_{\mathrm{c} 1}$ the superconductor is in the Meissner state, while for $H_{\mathrm{c} 1}<H<H_{\mathrm{c} 2}$ it is in the mixed state. The normal-conducting state is instead defined for $H>H_{\mathrm{c} 2}$. (b) Sketch of a side view of the magnetic flux structures present in the mixed state. . . . . . . . . . . . . . .

3.4 BCS surface resistance as a function of the mean free path for different values of reduced energy gap $\Delta / k T_{\mathrm{c}}$ (a), and for different values of temperature $T$ (b). The curves are calculated using the program SRIMP [2]. . . . . . . . . . . . . . . . . . . . . . . . . . . .

3.5 Comparison between experimental and theoretical data of the surface resistance as a function of the temperature [3] . . . . . . . .

4.1 Scheme of a single-cell cavity with the two antennas, input coupler and transmitted power probe, needed for the RF measurement. . . 
4.2 Pictures of: (a) the RF measurement system, (b) the three cryostat used for the cavities vertical test and (c) a 9-cells cavity mounted on the RF stand. This instrumentation is part of the vertical test facility at Fermilab. . . . . . . . . . . . . . . . . . . . . . . 43

4.3 Scheme of the vertical test RF calibration system. . . . . . . . . 45

4.4 Reflected power response in pulse mode, as a function of time, for different coupling regime. . . . . . . . . . . . . . . . . . . .

4.5 Example of Q-factor versus accelerating field curves acquired at $2 \mathrm{~K}$ and $1.5 \mathrm{~K}$. The red points are acquired in decay mode while all the other points are acquired in $\mathrm{CW}$ mode. . . . . . . . . . . . . . 48

4.6 Q-factor degradation due to Q-disease after a slow cooldown [4]. .

4.7 Q-factor versus accelerating field curves measured at $2 \mathrm{~K}$ for an EP and a $120^{\circ} \mathrm{C}$ baked cavity. . . . . . . . . . . . . . . . . . . .

4.8 Q-factor versus accelerating field curve measured at $2 \mathrm{~K}$ for a $\mathrm{N}$ doped and a $120{ }^{\circ} \mathrm{C}$ baked cavity. . . . . . . . . . . . . . . . . 55

4.9 Temperature (black curve) and pressure (blue curve) of the chamber during the $2 / 6$ nitrogen doping treatment. . . . . . . . . . . . .

4.10 SIMS spectra of a N-doped sample and some cut-outs from the cavity ACC002. Both sample and cut-outs are measured after a 20 minutes N-doping treatment. The cavity cut-outs were also electro-polished removing $5 \mu \mathrm{m}$ from the surface. . . . . . . . . . . . . . . . . .

4.11 Q-factor versus accelerating field curves, measured at $2 \mathrm{~K}$, of some of the cavities studied. . . . . . . . . . . . . . . . . . . . .

4.12 Surface resistance decomposition in BCS and residual resistance contributions for the cavity AES021. . . . . . . . . . . . . . . . .

5.1 Residual resistance at as a function of the thermal gradient $T_{1}-T_{2}$ between equator $T_{2}$ and top iris $T_{1}$ when the equator become SC. The inset shows the corresponding $Q_{0}$ values [5]. . . . . . . . . .

5.2 Sketch of the two cooldowns orientation: a) vertical and b) horizontal

5.3 Horizontal cooldown cavity set-up. The orange squares and the green rectangle represents respectively the thermometers and the fluxgates, while a and b stand for the two Helmholtz coils. . . . .

$5.4 Q_{0}$ versus accelerating field measured at $T<1.4 \mathrm{~K} . \quad$. . . . . . . 
5.5 Residual resistance versus mid-top (a) and bot-top (b) thermal gradients [6]. . . . . . . . . . . . . . . . . . . . . . . . . . . . . 68

5.6 Evolution of the SC-NC interface during the cooldown [6]. . . . . 69

5.7 Temperature variation versus the accelerating field [6]. . . . . . . 71

$5.8 Q_{0}$ and $T_{\text {top }}$ versus accelerating field for HTS measurements done with cooldowns started at $100 \mathrm{~K}$ and $60 \mathrm{~K}[6]$. . . . . . . . . . .

5.9 Ratio between the magnetic field measured after $\left(B_{\mathrm{SC}}\right)$ and before $\left(B_{\mathrm{NC}}\right)$ the $\mathrm{SC}$ transition for the axial magnetic field series. $B_{\mathrm{SC}} / B_{\mathrm{NC}}$ measured with the fluxgate at the cavity mid position (a) and with the fluxgate at the cavity top position (b). . . . . . . . . . . . .

5.10 Flux expulsion ratio $B_{\mathrm{SC}} / B_{\mathrm{NC}}$, for the orthogonal magnetic field series, measured with the fluxgate at the cavity mid position (a) and with the fluxgate at the cavity top position (b) [6]. . . . . .

5.11 COMSOL simulation of the magnetic field redistribution outside the cavity due to the Meissner effect, considering axial magnetic field applied. . . . . . . . . . . . . . . . . . . . . . . . . . . . .

5.12 a) COMSOL simulation of the magnetic field redistribution, along the yx-plane, considering orthogonal magnetic field applied (along the z-axis). b) COMSOL simulation of the magnetic field redistribution, along the zx-plane, considering orthogonal magnetic field applied (along the z-axis). . . . . . . . . . . . . . . . . . . .

5.13 Sketch of the field redistribution in the Meissner state with magnetic field applied a) axially and b) orthogonally to the cavity axis [6]. .

6.1 Ratio between the magnetic field after $B_{\mathrm{SC}}$ and before $B_{\mathrm{NC}}$ the superconducting transition as a function of the temperature difference

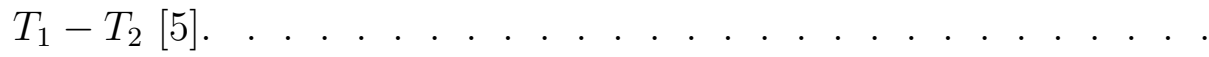

6.2 Gibbs free energy density $g$ as a function of the magnetic field $H$

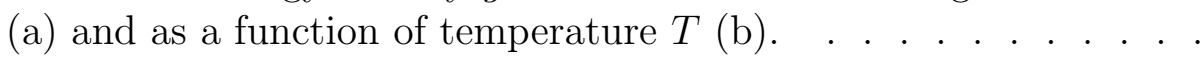

6.3 Critical current density as a function of the critical temperature difference $\Delta T_{\mathrm{c}}$. . . . . . . . . . . . . . . . . . . . . . . . . . . . . .

6.4 Gaussian probability density function as a function of the critical temperature difference $\Delta T_{\mathrm{c}}$. The orange area defines the probability that vortices are swiped out when the critical temperature difference $\Delta T_{\mathrm{c}_{\mathrm{i}}}$ is reached. . . . . . . . . . . . . . . . . . . . . . . . . .

6.5 Linear trend of the percentage of trapped field $\left(B_{\text {trap }}\right)$ versus the flux expulsion ratio $B_{\mathrm{SC}} / B_{\mathrm{NC}}$. . . . . . . . . . . . . . . . . . 
6.6 Probability density of expelling vortices as a function of the critical temperature difference for three example of probability density functions. . . . . . . . . . . . . . . . . . . . .

6.7 Flux expulsion ratio as a function of the temperature difference for the three example of probability density functions described in Figure 6.6. . . . . . . . . . . . . . . . . .

6.8 Probability density of expelling vortices as a function of the critical temperature difference along the cavity cell, for four example of probability density functions. . . . . . . . . . . . . . . . .

6.9 Flux expulsion ratio as a function of the temperature difference along the cavity cell, for the four example of probability density functions described in Figure 6.6. . . . . . . . . . . . . . . . . . . . . .

6.10 Comparison between the experimental values (yellow diamonds) and the simulated curve (blue curve) of the flux expulsion behavior of the cavity AES011. The red curve shows the probability density function used to generate the simulated curve. . . . . . . . . . .

6.11 Comparison between the experimental values (yellow dots) and the simulated curve (blue curve) of the flux expulsion behavior of the cavity CBMM. The red curve shows the probability density function used to generate the simulated curve. . . . . . . . . . . . . . .

6.12 Comparison between the experimental values (green squares and orange circles) and the simulated curves (blue curve) of the flux expulsion behavior of the cavities CBMM and ACC002. The simulation is done taking into account a double distribution of pinning centers.

6.13 Comparison between the experimental values (pink circles and yellow squares) and the simulated curves (blue curve) of the flux expulsion behavior of the cavities AES017 and AES017b. The simulation is done taking into account a double distribution of pinning centers.

6.14 Double peaked probability density functions used to generate the flux expulsion curves of the studied cavities. . . . . . . . . . .

6.15 Modeled flux expulsion behaviors of the studied cavities. . . . . . 100

6.16 Histogram representation of the pinning centers distribution (a) and probability density of expelling vortices (b) before and after the high temperature baking. . . . . . . . . . . . . . . . . .

6.17 Comparison between experimental (yellow diamonds) and simulated flux expulsion data (blue curve) of the cavity AES011 using a double peaked probability density function. . . . . . . . . . . . . . . 
6.18 Picture of: (a) the T-map system mounted on a single-cell cavity, (b) a single board of the T-map system mounted on the cavity.

6.19 (a) Sketch of a T-map image in which the temperature of the top and the bottom of the cavity equator are pointed out. The image also shows that the equator temperature is recorded with the thermometer number 8 . The white region of the T-map indicates a superconducting area with $T<T_{c}$, while the colored region indicates the normal-conducting area with $T>T_{c}$. (b) Scheme of a horizontal single-cell cavity in which the top, mid and bottom positions are indicated with the correspondent T-map board number. The scheme also shows how the 16 thermometers of one board are arranged around the cavity cell. . . . . . . . . . . . . . . . . . 105

6.20 T-map images recorded during a fast cooldown. . . . . . . . . . 106

6.21 T-map images recorded during a fast cooldown. . . . . . . . . . 107

6.22 T-map images recorded during a fast cooldown. . . . . . . . . . 108

6.23 T-map images recorded during a slow cooldown. . . . . . . . . . 110

6.24 T-map images recorded during a slow cooldown. . . . . . . . . . 111

6.25 T-map images recorded during a slow cooldown. . . . . . . . . . 112

6.26 T-map images recorded during a slow cooldown. . . . . . . . . . 113

6.27 T-map images recorded during a slow cooldown. . . . . . . . . . 114

6.28 T-map images recorded during a slow cooldown. . . . . . . . . . 115

6.29 Comparison between the T-map images acquired after fast cooldown in compensated field (no magnetic field trapped) and slow cooldown in $10 \mathrm{mG}$ (10 $\mathrm{mG}$ of magnetic field trapped). Both images are acquired on the cavity AES019 at about $27 \mathrm{MV} / \mathrm{m}$ and $1.5 \mathrm{~K}$. . .

6.30 Q-factor versus accelerating field curves acquired at $1.5 \mathrm{~K}$ for the cavity AES019 acquired after the cooldown with no field trapped (blue curve) and after that the cavity trapped about $10 \mathrm{mG}$ of magnetic field (pink curve). The T-map images of Figure 6.29 are acquired during the same measurements. . . . . . . . . . . . .

7.1 Scheme of the cavity instrumentation needed to monitor the external magnetic field and the cavity temperature during the cooldowns. The thermometers are represented as orange squares, while the green rectangles represent the fluxgates. The Helmholtz coil is indicated in the figure with the letter a. . . . . . . . . . . . . . . . . 
7.2 Interpolation using SRIMP of the penetration depth variation as a function of the temperature of the cavity AES011. . . . . . . . .

7.3 Penetration depth variation as a function of $y$ for some of the analyzed cavities, representative of different surface treatments. The connection line between the points serves just as guidance for the eyes. . . . . . . . . . . . . . . . . . . . . . . . . . .

7.4 Magnetic field penetration as a function of the depth, measured with the $\mu \mathrm{SR}$ technique for different cavity cut-outs. The measured magnetic field $B$ is normalized for the applied field $B_{\mathrm{a}}[7]$. . . . .

7.5 Comparison between cavities with same amount of trapped flux and different sensitivity values. . . . . . . . . . . . .

7.6 Trapped flux sensitivity calculated at $5 \mathrm{MV} / \mathrm{m}$ (orange diamonds) and $16 \mathrm{MV} / \mathrm{m}$ (green dots) as a function of the mean free path [8].

7.7 Sensitivity dependence on the accelerating field $[8] \ldots$. . . . . . .

7.8 Variation of the energy gap $\Delta$ as a function of the distance $r$ from the center of the vortex. The solid line shows the non-local description while the dashed line shown the local description $[9]$. . . . . . .

7.9 Sensitivity as a function of mean free path for the clean (black curve) and dirty (red curve) limits calculated considering dissipation coming from the normal-conducting core of the vortex. The yellow diamonds correspond to the experimental data at zero field. . . . . .

7.10 Normalized reduced magnetic filed $h(r) / h(0)$ and order parameter $\Psi(r)$ as a function of the radial distance from the center of vortex $r$ in the Abrikosov vortex description. . . . . . . . . . . . .

7.11 Sensitivity as a function of the mean free path calculated considering dissipation coming from the vortex oscillation due to RF field [10] (red curve) and comparison with experimental data at zero field (yellow diamonds). . . . . . . . . . . . . . . . . .

7.12 Sensitivity as a function of mean free path calculated from the Gurevich and Ciovati model [11]. The yellow diamonds correspond to the experimental data at zero field. . . . . . . . . . . . . . .

$7.132 \mathrm{~K}$ BCS surface resistance as a function of the accelerating field of some of the cavities analyzed. 
$7.142 \mathrm{~K}$ BCS surface resistance as a function of mean free path, at $5 \mathrm{MV} / \mathrm{m}$ (upper graph) and $16 \mathrm{MV} / \mathrm{m}$ (lower graph). The green diamonds represent doped cavities, while the pink circles represent non doped cavities [8]. . . . . . . . . . . . . . . . . . . . . . .

7.15 Q-factor at $2 \mathrm{~K}$ and $16 \mathrm{MV} / \mathrm{m}$ as a function of the mean free path. In order to make the graph as clear as possible, for the N-doped cavities only two points are shown. These two points represent the best and the worst case scenario among all the N-doped studied cavities [8]. . . . . . . . . . . . . . . . . . . . . . . . . . .

7.16 Q-factor at $2 \mathrm{~K}$ and $16 \mathrm{MV} / \mathrm{m}$ as a function of the trapped field for a $120^{\circ} \mathrm{C}$ bake, EP and 2/6 N-doped cavity. . . . . . . . . . .

7.17 Percentage of expelled flux needed to reach LCLS-II specification with $2 / 6 \mathrm{~N}$-doped cavities as a function of the remnant magnetic field in the cryomodule. . . . . . . . . . . . . . . . . . . . . . 


\section{LIST OF SYMBOLS}

\begin{tabular}{|c|c|}
\hline Symbol & Definition \\
\hline$c$ & Speed of light \\
\hline$e$ & Elementary charge \\
\hline$m$ & Electron mass \\
\hline$\epsilon_{0}$ & Vacuum dielectric constant \\
\hline$\mu_{0}$ & Vacuum magnetic permeability \\
\hline$E$ & Electric field \\
\hline$H$ & Magnetic field \\
\hline$B$ & Magnetic field induction \\
\hline$B_{\mathrm{e}}, H_{\mathrm{e}}$ & External magnetic field \\
\hline$v$ & Particle speed \\
\hline$\beta$ & Ratio of the particle speed divided to $c$ \\
\hline$\hat{n}$ & Unit vector perpendicular to the surface \\
\hline$\omega_{0}$ & Angular resonance frequency \\
\hline$J_{\mathrm{n}}$ & n-th Bessel function \\
\hline$\alpha_{\mathrm{nl}}$ & l-th zero of the $\mathrm{n}$-th Bessel function \\
\hline$\alpha_{\mathrm{nl}}$ & 1-th zero of the $\mathrm{n}$-th Bessel function \\
\hline$U$ & Stored energy \\
\hline$P_{\mathrm{c}}$ & Cavity dissipated power \\
\hline$Q_{0}$ & Unloaded quality factor \\
\hline$G$ & Geometry constant \\
\hline$E_{\mathrm{pk}}, B_{\mathrm{pk}}$ & Electric and magnetic field peak \\
\hline
\end{tabular}




\begin{tabular}{|c|c|}
\hline$R_{\mathrm{a}}$ & Shunt impedance \\
\hline$r_{\mathrm{a}}$ & Shunt impedance per unit of length \\
\hline$R_{\mathrm{s}}$ & Surface resistance \\
\hline$n$ & Density of charge carriers \\
\hline$n_{\mathrm{n}}$ & Density of normal-electrons \\
\hline$n_{\mathrm{s}}$ & Density of super-electrons \\
\hline$\lambda_{\mathrm{L}}$ & London penetration depth \\
\hline$J$ & Current density \\
\hline$J_{\mathrm{n}}$ & Normal-electrons current density \\
\hline$J_{\mathrm{s}}$ & Super-electrons current density \\
\hline$H_{\mathrm{c}}$ & Critical field \\
\hline$T_{\mathrm{c}}$ & Critical temperature \\
\hline$\Delta$ & Energy gap \\
\hline$\xi$ & Coherence length \\
\hline$\lambda$ & Penetration depth \\
\hline$\psi$ & Order parameter \\
\hline$\kappa$ & Ginzburg-Landau parameter \\
\hline$\Phi_{0}$ & Magnetic flux quantum \\
\hline$N(0)$ & Density of state at the Fermi level \\
\hline$\sigma_{1}$ & Real part of the conductivity \\
\hline$\sigma_{2}$ & Imaginary part of the conductivity \\
\hline$Z_{\mathrm{s}}$ & Surface impedance \\
\hline
\end{tabular}




\begin{tabular}{|c|c|}
\hline$Z_{\mathrm{n}}$ & Normal-conducting surface impedance \\
\hline$R_{\mathrm{BCS}}$ & BCS surface resistance \\
\hline$R_{\text {res }}$ & Residual resistance \\
\hline$R_{0}$ & Intrinsic residual resistance \\
\hline$R_{\mathrm{n}}$ & Normal-conducting surface resistance \\
\hline$R_{\mathrm{f}}$ & Trapped flux surface resistance \\
\hline$\eta$ & Flux trapping efficiency or Carnot efficiency \\
\hline$S$ & Trapped flux sensitivity \\
\hline$P_{\mathrm{e}}$ & Emitted power \\
\hline$P_{\mathrm{t}}$ & Transmitted power \\
\hline$P_{\text {in }}$ & Incident power \\
\hline$P_{\mathrm{f}}$ & Forward power \\
\hline$Q_{\mathrm{L}}$ & Load Q-factors \\
\hline$\beta_{1}$ & Input coupler coupling strength \\
\hline$\beta_{2}$ & Output coupler coupling strength \\
\hline$Q_{1}$ & Input coupler Q-factor \\
\hline$Q_{2}$ & Output coupler Q-factor \\
\hline$\Gamma$ & Reflection coefficient or Anisotropic parameter \\
\hline$\rho$ & Resistivity \\
\hline$R R R$ & Residual resistivity ratio \\
\hline$E_{\text {acc }}$ & Accelerating field \\
\hline$N_{\text {trap }}$ & Number of trapped vortices \\
\hline
\end{tabular}




$\begin{array}{cl}g & \text { Gibbs free energy density } \\ H_{\mathrm{c} 1} & \text { Lower critical field } \\ f_{\mathrm{p}} & \text { Pinning force } \\ \nabla T_{\mathrm{c}} & \text { Critical thermal gradient } \\ \Delta T_{\mathrm{c}} & \text { Critical temperature difference along the cavity } \\ P\left(\nabla T_{\mathrm{c}}\right) & \left.\text { Probability of expelling vortices at } \nabla T_{\mathrm{c}}\right) \\ B_{\mathrm{SC}} / B_{\mathrm{NC}} & \text { Flux expulsion ratio } \\ B_{\mathrm{SC}} & \text { Magnetic field after the SC transition } \\ B_{\mathrm{NC}} & \text { Magnetic field before the SC transition } \\ \ell & \text { Mean free path } \\ f_{\mathrm{p}} & \text { Viscosity } \\ f_{\mathrm{c}} & \text { Super-electrons critical momentum } \\ a & \text { Normal-conducting core vortex radious } \\ f_{\mathrm{L}} & \text { Lorentz force }\end{array}$




\begin{abstract}
Accelerating cavities are devices resonating in the radio-frequency $(\mathrm{RF})$ range used to accelerate charged particles in accelerators. Superconducting accelerating cavities are made out of niobium and operate at the liquid helium temperature. Even if superconducting, these resonating structures have some RF driven surface resistance that causes power dissipation. In order to decrease as much as possible the power losses, the cavity quality factor must be increased by decreasing the surface resistance.

In this dissertation, the RF surface resistance is analyzed for a large variety of cavities made with different state-of-the-art surface treatments, with the goal of finding the surface treatment capable to return the highest Q-factor values in a cryomodule-like environment. This study analyzes not only the superconducting properties described by the BCS surface resistance, which is the contribution that takes into account dissipation due to quasi-particle excitations, but also the increasing of the surface resistance due to trapped flux. When cavities are cooled down below their critical temperature inside a cryomodule, there is always some remnant magnetic field that may be trapped increasing the global RF surface resistance.
\end{abstract}

This thesis also analyzes how the fraction of external magnetic field, which is actually trapped in the cavity during the cooldown, can be minimized. This study is performed on an elliptical single-cell horizontally cooled cavity, resembling the geometry of cavities cooled in accelerator cryomodules. The horizontal cooldown study reveals that, as in case of the vertical cooldown, when the cooling is performed fast, large thermal gradients are created along the cavity helping magnetic flux expulsion. However, for this geometry the complete magnetic flux expulsion from the cavity equator is more difficult to achieve. This becomes even more challenging in presence of orthogonal magnetic field, that is easily trapped on top of the cavity equator causing temperature rising. 
The physics behind the magnetic flux expulsion is also analyzed, showing that during a fast cooldown the magnetic field structures, called vortices, tend to move in the same direction of the thermal gradient, from the Meissner state region to the mixed state region, minimizing the Gibbs free energy. On the other hand, during a slow cool down, not only the vortices movement is limited by the absence of thermal gradients, but, also, at the end of the superconducting transition, the magnetic field concentrates along randomly distributed normal-conducting region from which it cannot be expelled anymore.

The systematic study of the surface resistance components performed for the different surface treatments, reveals that the BCS surface resistance and the trapped flux surface resistance have opposite trends as a function of the surface impurity content, defined by the mean free path. At medium field value, the BCS surface resistance is minimized for nitrogen-doped cavities and significantly larger for standard niobium cavities. On the other hand, Nitrogen-doped cavities show larger dissipation due to trapped flux. This is consequence of the bell-shaped trend of the trapped flux sensitivity as a function of the mean free path. Such experimental findings allow also a better understanding of the RF dissipation due to trapped flux.

The best compromise between all the surface resistance components, taking into account the possibility of trapping some external magnetic field, is given by light nitrogen-doping treatments. However, the beneficial effects of the nitrogen-doping is completely lost when large amount of magnetic field is trapped during the cooldown, underlying the importance of both cooldown and magnetic field shielding optimization in high quality factors cryomodules. 


\section{CHAPTER 1 \\ INTRODUCTION}

Modern particle accelerators employ superconducting resonators in order to accelerate charged particle up to speed very close to the speed of light.

The recent discovery of the Higgs boson by the Large Hadron Collider (LHC) at the European Organization for Nuclear Research (CERN) [12, 13], is just the last demonstration on how important are accelerators to answer the most fundamental questions of our nature and our universe.

Accelerators are not useful only for fundamental physics research, which brought about $30 \%$ of the Nobel prizes in physics [14], but also as synchrotron radiation sources, needed for material science and biological experiment, for medial applications, providing hadron beams for radiation therapy or production of isotopes, for food sterilization, and so on $[15,16,17,18]$.

Particle accelerators are, however, really expensive machines and sometimes their high prohibitive costs prevent their realization. In order to avoid that, it is very important to improve the technology behind accelerators in order to cut down as much as possible both the capital and the operational cost of these machines.

\subsection{The importance of high Q-factors for particle accelerators}

Taking into account accelerating cavities, the employment of the superconducting technology allows to provide an affordable technology for continuous wave (CW) and high duty cycle accelerators. The decreasing of the dissipated power in superconducting cavities is given by the several order of magnitude lower surface resistance, compared with normal-conducting cavities.

The accelerating field, or accelerating gradient, is proportional to the energy 
gained by the charged particle during each passage through the cavity. The large dissipated power in normal-conducting cavities limits their usage, in CW mode, at accelerating gradient of about $1 \mathrm{MV} / \mathrm{m}$, while the current limit for the superconducting technology is about $45 \mathrm{MV} / \mathrm{m}$. Therefore, the lower dissipation of superconducting cavities is really important in order to build more efficient and powerful accelerators.

The quality factor $Q_{0}$, or Q-factor, is the parameter that indicates the power dissipation in accelerating cavities, being defined as:

$$
Q_{0}=\frac{G}{R_{\mathrm{s}}}=\frac{\omega_{0} U}{P_{\mathrm{c}}}
$$

where $G$ is a geometrical constant, $R_{\mathrm{s}}$ the surface resistance, $\omega_{0}$ the angular resonance frequency, $U$ the stored energy and $P_{\mathrm{c}}$ is the power dissipated in the cavity walls. Low values of surface resistance defines low dissipated power and high Q-factor.

When quality factors are maximized, the power consumption needed to reach a certain level of accelerating field is lowered, decreasing the operational costs. In addition, the possibility to reach larger values of accelerating field means that a smaller number of cavities may be used to make the beam reach a certain energy. This means that increasing the Q-factors the operational cost of accelerators may be reduced as well.

For the reason above, the $R \& D$ of superconducting cavities has always been focused on the improving of Q-factors and accelerating fields. Superconducting cavities operating in accelerators are usually made out of bulk niobium, and in order to improve the superconducting performance, the optimization of the first hundreds of nanometers of the cavity surface is needed. Thanks to the R\&D efforts, the superconducting cavities performance improved considerably in the last years, gaining a final boost with the discovery of the nitrogen-doping treatment in 2013 [19]. Nitrogendoped elliptical TESLA-type cavities [3] can easily reach Q-factor of $5 \cdot 10^{10}$ at 2 
$\mathrm{K}$ and $16 \mathrm{MV} / \mathrm{m}$, which is three times larger than the Q-factor achievable with the standard niobium technology [19]. For a CW machine this traduces in a minimization of the SRF cryogenic cost of more than $50 \%$ [20].

During the same period of the discovery of the nitrogen-doping, SLAC was working on the construction concept of the upgrading of its Linac Coherent Ligth Source (LCLS-II). Under suggestion of the US DOE, they were considering the construction of a CW SRF LINAC in the existing SLAC tunnel. Therefore SLAC invested in the nitrogen-doping technology with the purpose of supporting a rapid development of procedures capable of minimizing LCLS-II cryogenic heat load. Fermilab, JLAB and Cornell University joined the LCLS-II collaboration working on the R\&D to make the nitrogen-doping a reliable technology capable to systematically provide high-Q 9-cells TESLA-type cavities [20]. Part of this thesis work is performed in the framework of this collaboration.

\subsection{Thesis goals and organization}

Even though the nitrogen-doping treatments immediately shows very high values of quality factors, it also reveals larger dissipations due to trapped flux $[8,21$, $22,23]$. This dissipation is due to magnetic field that may be trapped in the cavity walls during the superconducting transition.

Purpose of this thesis work is to clarify how the high Q-factors given by the nitrogen-doping treatment can be achieved and preserved when cavities are placed inside a cryomodule and operate in the accelerator.

Together with this practical purpose, also some fundamental aspects behind the physics of the magnetic flux trapping, the nitrogen-doping superconductivity enhancement and the trapped flux dissipation are revealed.

Chapter 2 shows an overview on radio-frequency accelerating cavities, how 
they work and the figures of merit needed to understand their performance. The elliptical TESLA-type cavities are here described, clarifying the nomenclature of their different part which is used in the rest of the thesis. The Chapter ends underlying the importance of the superconducting technology, needed to provide an affordable method of acceleration especially for CW and high duty cycle accelerators.

An overview of the main theories of superconductivity is shown in Chapter 3. The definitions of the Mattis and Bardeen surface resistance, called BCS surface resistance and the residual resistance contributions are explained at the end of the chapter. These two components of the surface resistance are further analyzed in the other chapters of the thesis.

Superconducting cavities are characterized by performing RF measurements in which the power dissipated in the cavity walls is calculated from a power balance that takes into account the power forwarded, reflected and transmitted to and from the cavity, as explained in Chapter 4 . In the same chapter the main steps of cavities fabrication are explained, underlying the importance of the final surface treatment on the cavity performance. The state-of-the-art surface treatments are therefore described, from the standard niobium treatments to the recently discovered nitrogendoping treatment. A summary of the surface treatments done on the cavities studied through the thesis work is presented with a brief analysis on their surface resistance properties.

Chapters 5 and 6 are focused on the study and understanding of the magnetic flux trapping in superconducting cavities during the cooldown below their critical temperatures. When magnetic field is trapped during the superconducting transition, an additional term on the surface resistance appear, called trapped flux surface resistance $R_{f l}$. This surface resistance contribution takes into account losses due to 
trapped flux and is defined by:

$$
R_{\mathrm{fl}}=\eta \cdot B_{\mathrm{ext}} \cdot S
$$

where $\eta$ is the flux trapping efficiency that multiplied by the external magnetic field $B_{\text {ext }}$ gives the amount of magnetic field trapped in the cavity $B_{\text {trap }}$, and $S$ is the trapped flux sensitivity which indicates the resistance for unit of trapped field. Chapter 5 and 6 focus on the understanding of the flux trapping efficiency $\eta$ which depends on how the cavity cooldown is performed. In particular, in Chapter 5 the cooldown is studied for a single-cell cavity horizontally cooled, resembling the cooldown geometry of cavities in accelerators. The study is performed for both axial and orthogonal magnetic field applied, revealing that the magnetic field orientation matters and, under specific condition, magnetic field may be trapped on top of the cavity equator as a consequence of the orientation between cavity cooling and magnetic field. In Chapter 6 instead, the physics behind the magnetic flux expulsion is studied, explaining the mechanisms of efficient flux expulsion during fast cooldowns and full flux trapping during slow cooldowns.

The last experimental chapter of the thesis, Chapter 7, shows the systematic study of the surface resistance contributions of several cavities treated with standard niobium treatments and several different nitrogen-doping recipes. Both the trapped flux sensitivity $S$ and the BCS surface resistance $R_{\mathrm{BCS}}$ are studied as a function of the mean free path and of the accelerating field. This analysis allows to clarify which is the surface treatment capable to maximize the quality factor even in presence of trapped flux, therefore considering a realistic situation comparable with the cavities in the cryomodule. These results also provide new insights on the physics behind the change in the field dependence of the Mattis and Bardeen surface resistance between different surface treatments, and of the trapped magnetic vortices induced losses in superconducting niobium resonators. 
This experimental work allows to define all the ingredients needed to successfully obtain high Q-factor, in the SRF modules of accelerators, by implementing the N-doping treatment. 


\section{CHAPTER 2}

\section{RADIO-FREQUENCY ACCELERATING CAVITIES}

\subsection{Accelerating cavities}

Resonant radio-frequency $(\mathrm{RF})$ cavities are devices capable to store electromagnetic energy and are used to accelerate charged particles in accelerators. Depending on their nature, particles may reach from small fraction of the speed of light, as for heavy ions, to values very close to the speed of light in case of electrons, positrons and so on.

Accelerating cavities may have different shape and fundamental resonance frequency, depending on their application. Usual resonance frequencies for accelerating cavities are within the RF range, in particular between $100 \mathrm{MHz}$ and $10 \mathrm{GHz}$.

The simplest example of such a resonator is the pill-box cavity, which is a cylindrical wave-guide closed with metallic plates at both the two ends. Thanks to its easy shape, the spatial distribution of the electromagnetic field can be calculated analytically [24].

Considering a pill-box with length $h$ and radius $a$ (Figure 2.1), made out of a perfect conductor and filled with a lossless dielectric, the electromagnetic field distribution must satisfy both the wave equation:

$$
\left(\nabla^{2}-\frac{1}{c^{2}} \frac{\partial^{2}}{\partial t^{2}}\right)\left\{\begin{array}{c}
E \\
H
\end{array}\right\}=0
$$

and the boundary conditions:

$$
\hat{n} \times \bar{E}=0 \quad, \quad \hat{n} \cdot \bar{H}=0,
$$

where $c$ is the speed of light, $\hat{n}$ is the unit vector normal to the surface, and $E$ and $H$ the electric and magnetic field respectively. The boundary conditions assure that 


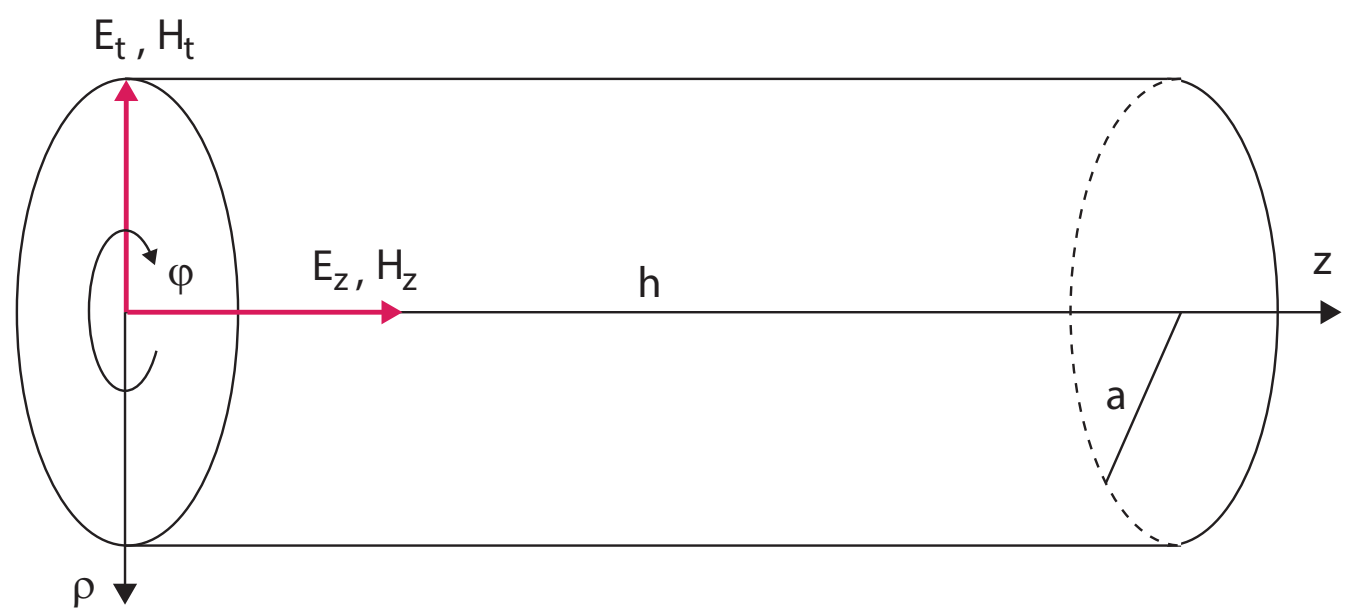

Figure 2.1. Sketch of a pill-box cavity with radius $a$ and length $h$.

the tangential component of the electric field E, and the normal component of the magnetic field $\mathrm{H}$ are continuous across the interface between the dielectric and the conductor.

Because of the cylindrical symmetry of the problem it is useful to consider a cylindrical system of coordinates in which the fields can be decomposed in their longitudinal $\left(E_{\mathrm{z}}\right.$ and $\left.H_{\mathrm{z}}\right)$ and transverse components $\left(E_{\mathrm{t}}\right.$ and $\left.H_{\mathrm{t}}\right)$ as:

$$
\begin{aligned}
& E(\rho, \phi, z, t)=E_{\mathrm{z}}(\rho, \phi, z, t)+E_{\mathrm{t}}(\rho, \phi, z, t), \\
& H(\rho, \phi, z, t)=H_{\mathrm{z}}(\rho, \phi, z, t)+H_{\mathrm{t}}(\rho, \phi, z, t),
\end{aligned}
$$

where $\rho$ is the radial distance, $\phi$ is the azimuthal angle and $z$ is the distance along the z-axis, as indicated in Figure 2.1. The wave-equation (Equation 2.1) is therefore solved for the longitudinal component of the fields $E_{\mathrm{z}}$ and $H_{\mathrm{z}}$.

Because of the different boundary conditions for $E_{z}$ and $H_{z}$ :

$$
\left.E_{\mathrm{z}}\right|_{\mathrm{S}}=0 \quad,\left.\quad \hat{\mathrm{n}} \frac{\partial H_{\mathrm{z}}}{\partial \hat{\mathrm{n}}}\right|_{\mathrm{S}}=0,
$$

where $S$ is the surface of the pill-box, the solution of Equation 2.1 defines two different types of modes. One is the transverse-electric mode $\left(T E_{\mathrm{nlm}}\right)$, in which the electromagnetic wave has only the electric field along the cavity axis, and the other is the 
transverse-magnetic mode $\left(T M_{\mathrm{nlm}}\right)$ in which the magnetic field is the only component along the cavity axis.

The indexes $n, l$, and $m$ are integers that identify the number of zeros along the $\phi, \rho$, and $z$ directions respectively.

In order to accelerate a charged particle beam, electric field along the direction of propagation of the beam is needed. For this reason the only modes useful at this purpose are the $T M_{\mathrm{nlm}}$. Considering that also the end surfaces of the pill-box, placed at $z=0$ and $z=h$, are made of perfect conductor, the complete set of $T M_{\mathrm{nlm}}$ modes is given by [24]:

$$
\begin{aligned}
& E_{\mathrm{z}}=E_{0} J_{n}\left(\frac{\alpha_{\mathrm{nl}}}{a} \rho\right) \cos \left(\frac{m \pi z}{h}\right) \cos (n \varphi) e^{ \pm i \omega_{\mathrm{nlm}} t} \\
& E_{\rho}=-\frac{m \pi a}{h \alpha_{\mathrm{nl}}} E_{0}\left[\left(\frac{n a}{\rho \alpha_{n l}}\right) J_{\mathrm{n}}\left(\frac{\alpha_{n l}}{a} \rho\right)-J_{\mathrm{n}+1}\left(\frac{\alpha_{\mathrm{nl}}}{a} \rho\right)\right] \\
& \qquad \cdot \sin \left(\frac{m \pi z}{h}\right) \cos (n \varphi) e^{ \pm i \omega_{\mathrm{nlm}} t} \\
& E_{\varphi}=\frac{m n \pi a^{2}}{\rho h \alpha_{\mathrm{nl}}^{2}} E_{0} J_{n}\left(\frac{\alpha_{\mathrm{nl}}}{a} \rho\right) \cos \left(\frac{m \pi z}{h}\right) \sin (n \varphi) e^{ \pm i \omega_{\mathrm{nlm}} t} \\
& H_{\mathrm{z}}=0 \\
& H_{\rho}=\frac{i \varepsilon_{0} \omega_{n l m} n a^{2}}{\rho \alpha_{\mathrm{nl}}^{2}} E_{0} J_{\mathrm{n}}\left(\frac{\alpha_{\mathrm{nl}}}{a} \rho\right) \cos \left(\frac{m \pi z}{h}\right) \sin (n \varphi) e^{ \pm i \omega_{\mathrm{nlm}} t} \\
& H_{\varphi}=\frac{i \varepsilon_{0} \omega_{\mathrm{nlm}} a}{\alpha_{\mathrm{nl}}} E_{0}\left[\left(\frac{n a}{\rho \alpha_{n l}}\right) J_{\mathrm{n}}\left(\frac{\alpha_{\mathrm{nl}}}{a} \rho\right)-J_{\mathrm{n}+1}\left(\frac{\alpha_{\mathrm{nl}}}{a} \rho\right)\right] \\
& \quad \cdot \cos \left(\frac{m \pi z}{h}\right) \cos (n \varphi) e^{ \pm i \omega_{\mathrm{nlm}} t},
\end{aligned}
$$

where $\varepsilon_{0}$ is the vacuum permittivity and $\omega_{\text {nlm }}$ is the angular resonance frequency:

$$
\omega_{\mathrm{nlm}}=c \sqrt{\left(\frac{\alpha_{\mathrm{nl}}}{a}\right)^{2}+\left(\frac{m \pi}{h}\right)^{2}}
$$

where $\alpha_{\mathrm{nl}}$ identifies the $l$-th zero of the $n$-th Bessel function $J_{\mathrm{n}}\left(\frac{\alpha_{\mathrm{nl}}}{a} \rho\right)$ and $n=$ $0,1,2 \ldots, l=1,2,3 \ldots, m=0,1,2 \ldots$

The lowest $T M$ mode is therefore the $T M_{010}$. Its resonance frequency is:

$$
\omega_{010}=\frac{2.405 c}{a}
$$



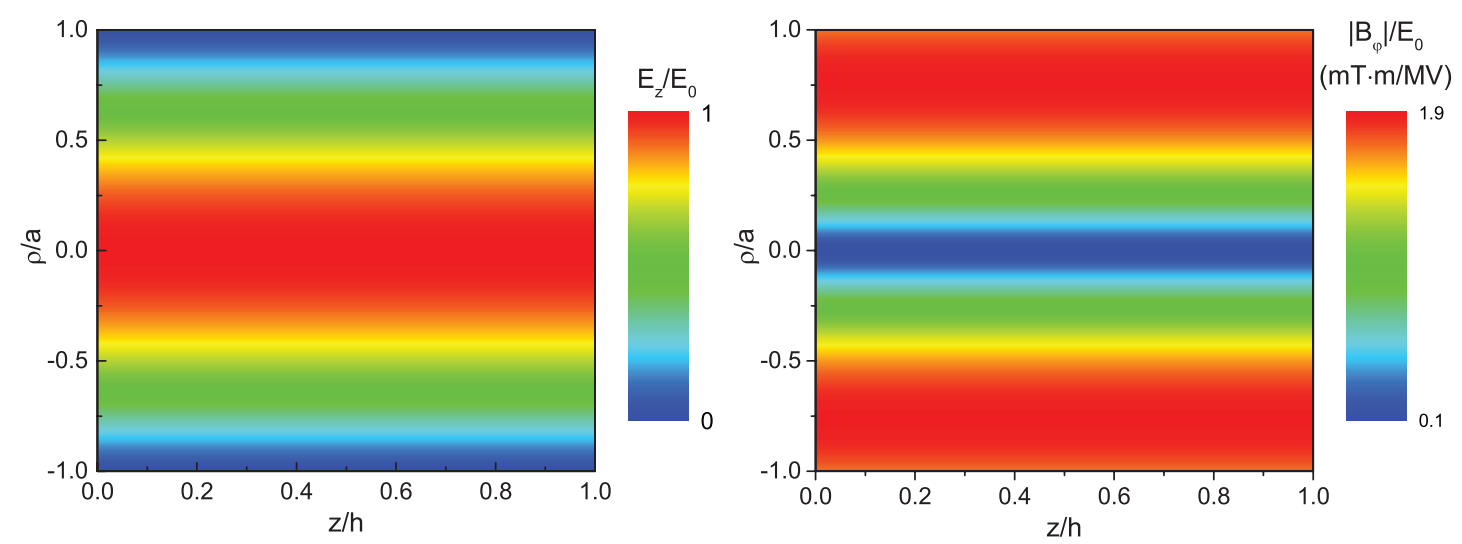

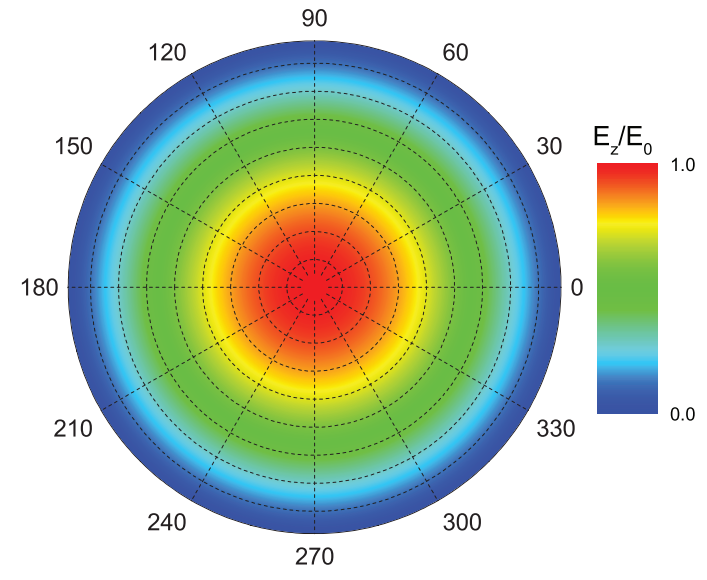

(a)

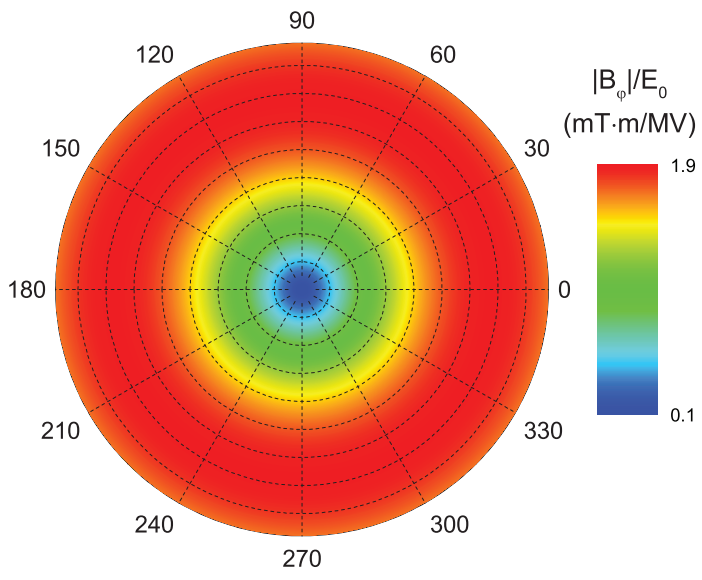

(b)

Figure 2.2. Axial (top) and polar (bottom) plots of the field $E_{\mathrm{z}}$ (a) and $B_{\phi}$ that resonates in the $\mathrm{TM}_{010}$ mode inside a pill-box. Both the fields are normalized for the electric field amplitude $E_{0}$.

and the fields are:

$$
\begin{aligned}
& E_{\mathrm{z}}=E_{0} J_{0}\left(\frac{2.405}{a} \rho\right) e^{ \pm i \omega_{010} t} \\
& H_{\varphi}=-i \varepsilon_{0} c E_{0} J_{1}\left(\frac{2.405}{a} \rho\right) e^{ \pm i \omega_{010} t}
\end{aligned}
$$

where $\alpha_{01}=2.405$ corresponds to the first zero of the zeroth Bessel function. The distribution of the fields in the $T M_{010}$ is shown in Figure 2.2. From this figure it is clear that for this mode the electric field is maximum at the center of the pill-box and decreases approaching the conducting walls at which it vanishes. On the other hand, the magnetic field is zero at the center of the pill-box and it reaches its maximum value really close to the pill-box wall, specifically at $\rho=0.77 a$. 
the set of $T M_{0 \mathrm{nl}}$ modes, called monopole modes, are the only one that can be used to accelerate particles since the electric field does not vanish along the beam axis. Indeed only the zeroth Bessel function goes to zero for $\rho=0$. Usually the $T M_{010}$ mode is preferred since it has the lowest eigenfrequency.

In order to let the beam being accelerated, holes and beam pipes have to be added at the two ends of the cylinder. Since the beam pipes act as wave-guides, their dimension has to be chosen so that their cut-off frequency is above the frequency of the mode used for acceleration. The holes in the pill-box walls act as perturbation for the electromagnetic field making difficult to solve analytically the wave equation, therefore computer codes are usually needed for this purpose [25, 26].

\subsection{Figure of merits}

2.2.1 The accelerating field. The accelerating field $E_{\text {acc }}$ is the field that provide the acceleration of charged particle and it is defined as the accelerating voltage $V_{\text {acc }}$ divided by the accelerating gap $d$ (which for the pill-box case corresponds to its length). The accelerating voltage is given by the integral of the electric field seen by the particle which is passing through the cavity. Considering the acceleration of an electron in a pill-box operating in the $T M_{010}$ mode, $V_{\text {acc }}$ is defined as:

$$
V_{\mathrm{acc}}=E_{0}\left|\int_{0}^{d} e^{i \omega_{0} z / c} d z\right|=d E_{0} \frac{\sin \left(\frac{\omega_{0} d}{2 c}\right)}{\frac{\omega_{0} d}{2 c}}=d E_{0} T,
$$

where $T$ is the transit-time factor, which is a measure of the reduction in energy gain caused by the sinusoidal time variation of the field in the accelerating gap. Therefore, in order to gain the maximum acceleration, the condition $\frac{\omega_{0} d}{2 c}=\frac{\pi}{2}$ has to be satisfy. This corresponds to:

$$
\frac{d}{v}=\frac{T_{\mathrm{RF}}}{2}
$$

where $T_{\mathrm{RF}}=\frac{2 \pi}{\omega_{0}}$ is the period of oscillation of the RF field. This condition sets that the particle with velocity $v$ has to be synchronous with the RF field in the cavity. In 
this case the accelerating field results $E_{\text {acc }}=2 E_{0} / \pi$.

2.2.2 The Quality Factor. In the previous section a perfect conducting behavior of the resonant cavity was assumed. In reality, even superconducting materials have some losses when exposed to micro-wave field, therefore the electromagnetic energy $U$ stored in the cavity exponentially decays with time, instead of remaining constant. The number of RF periods needed to consume the energy $U$ are related to the cavity quality factor $Q_{0}$ :

$$
U=U_{0} e^{-\frac{\omega_{0} t}{Q_{0}}}
$$

Since the dissipated power $P_{\mathrm{c}}$ is defined as the negative change of the stored energy $U$ with the time, $P_{\mathrm{c}}$ is inversely proportional to $Q_{0}$ :

$$
P_{\mathrm{c}}=\frac{\omega_{0} U}{Q_{0}} .
$$

Now, taking into account that the energy stored in the resonator is defined as:

$$
U=\frac{1}{2} \mu_{0} \int_{V}|\bar{H}|^{2} d v=\frac{1}{2} \varepsilon_{0} \int_{V}|\bar{E}|^{2} d v,
$$

where $V$ is the cavity volume, $d v$ is the volume element and the dissipated power is defined as:

$$
P_{\mathrm{c}}=\frac{1}{2} R_{\mathrm{s}} \int_{S}|\bar{H}|^{2} d s,
$$

where $S$ is the cavity surface and $d s$ is the surface element, the quality factor may be defined as:

$$
Q_{0}=\frac{\frac{1}{2} \mu_{0} \int_{V}|\bar{H}|^{2} d v}{\frac{1}{2} R_{\mathrm{s}} \int_{S}|\bar{H}|^{2} d s}=\frac{G}{R_{\mathrm{s}}},
$$

where $G$ is the geometrical factor, which is a constant value that depends only on the cavity geometry and does not depend on its size. In order to bring $R_{s}$ outside the integral, the surface resistance is assumed to not vary over the cavity surface.

The quality factor therefore depends on the cavity geometry and is inversely proportional to the surface resistance. For accelerating cavities $Q_{0}$ is of crucial im- 
portance since defines the power dissipated from a cavity during its operation in the accelerator.

2.2.3 Other figures of merit. Other figures of merit of accelerating cavities are useful in order to compare and optimize cavity shapes, which vary depending on the accelerator application.

The maximum accelerating field achievable at the surface of the cavity is defined by $E_{\mathrm{pk}} / E_{\mathrm{acc}}$ and $H_{\mathrm{pk}} / E_{\mathrm{acc}}$, where $E_{\mathrm{pk}}$ and $H_{\mathrm{pk}}$ are the peak surface electric and magnetic field, respectively. Taking into account the $T M_{010}$ mode resonating in a pill-box cavity, from Equation 2.8 the peak fields are:

$$
E_{\mathrm{pk}}=E_{0} \quad, \quad H_{\mathrm{pk}}=\varepsilon_{0} c E_{0} J_{1}(1.84),
$$

being $E_{\mathrm{pk}}$ on axis $(\rho=0)$ and $H_{\mathrm{pk}}$ at $\rho=0.77 a$. The ratios therefore are:

$$
\frac{E_{\mathrm{pk}}}{E_{a c c}}=\frac{\pi}{2}=1.6 \quad, \quad \frac{H_{\mathrm{pk}}}{E_{a c c}}=\frac{\pi}{2}=2430 \frac{A / m}{M V / m} .
$$

The shunt impedance $R_{a}$ is another important parameter correlated to the power dissipated in the cavity wall and it is defined in three different ways:

$$
R_{\mathrm{a}}=\frac{V_{a c c}^{2}}{P_{\mathrm{c}}} \quad, \quad R_{\mathrm{a}}^{\mathrm{c}}=\frac{V_{\mathrm{acc}}^{2}}{2 P_{\mathrm{c}}} \quad, \quad r_{\mathrm{a}}^{\mathrm{c}}=\frac{V_{\mathrm{acc}}^{2}}{P_{\mathrm{c}}^{\prime}},
$$

where $R_{\mathrm{a}}^{\mathrm{c}}$ is the definition used in circuit theory, $r_{a}^{c}$ is the definition for linear accelerators (LINACs) and $P_{\mathrm{c}}^{\prime}$ is the power dissipated per unit length. In order to minimize the dissipated power to reach a certain voltage across the cavity gap, the shunt impedance has to be maximized.

The ratio between the shunt impedance and the Q-factor, called $r$ over $q$, is defined as:

$$
\frac{R_{\mathrm{a}}}{Q}=\frac{V_{\mathrm{c}}^{2}}{\omega_{0} U}
$$

and is useful to measure how efficient is the cavity to reach a certain voltage across the cavity gap per unit of stored energy, for a given frequency $[25,27]$. 


\subsection{Cavities classification}

Accelerating cavities may have different shape, size and therefore fundamental resonance frequency. The choice of the design is mainly dictated by the type of particles that have to be accelerated, the speed they have before the acceleration and the desired speed after the acceleration. Cavities are therefore classified depending on the speed that particles reach after being accelerated, and it is possible to distinguish between low- $\beta$, medium- $\beta$ and high- $\beta$ resonators, where $\beta=v / c$.

Low- $\beta$ cavities $(\beta \simeq 0.001-0.2)$ are used to accelerate heavy-ions, while medium- $\beta$ cavities $(\beta \simeq 0.2-0.7)$ are used for protons with non relativistic energy and ions, and high- $\beta$ cavities $(\beta \simeq 1$ ) are used for electrons, positrons and high energy protons.

Material R\&D is nowadays focused principally on high- $\beta$ structure, since the most relevant for the final energy of the accelerated particle in synchrotron and electrons (or positrons) LINACs. These cavities are elliptically shaped and usually coupled in a chain of multi-cell in order to maximize the accelerating gradient per unit of length.

During the last decade the R\&D has been particularly focused on TESLAtype elliptical cavities [3], which are 9-cells $1.3 \mathrm{GHz}$ cavities that operates in the $T M_{010}$ mode. The intensive $\mathrm{R} \& \mathrm{D}$ had the main purpose of delivering an affordable technology capable to fulfill the requirements for the International Linear Collider (ILC) cryomodules. While this project has bot been fully financed yet, the SRF technology originated from it was implemented in the cryomodule production of the Free Electron LaSer (FLASH), the European X-ray Free Electron Laser (XFEL) both in Hamburg and the Light Coherent Luminosity Source superconducting linac (LCLSII) at SLAC. 


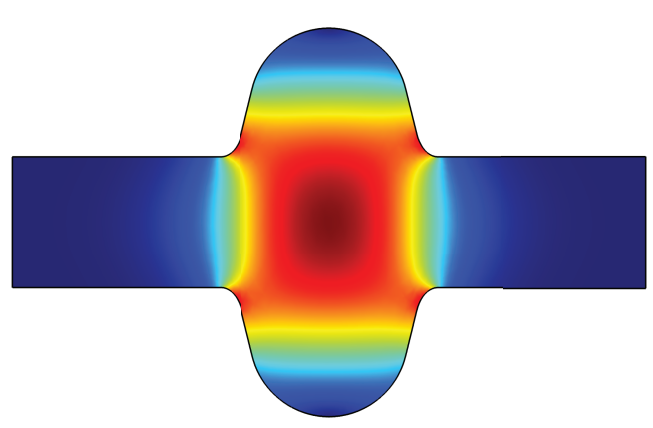

(a)

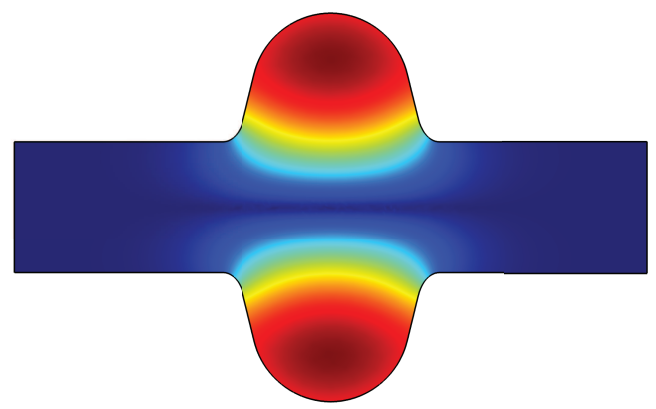

(b)

Figure 2.3. Electric a) and magnetic b) field density in an elliptical $1.3 \mathrm{GHz}$ Tesla type cavity. The dark red region indicates the highest field density, while the dark blue region indicates the lowest.

Because of the large cost of a 9-cells cavity, the R\&D is usually initially focused on single-cell cavities.

The electromagnetic field distribution of a TESLA-type cavity is similar to the one described previously for the pill-box cavity, having the maximum of the electric field along the beam axis, where the magnetic field is minimum, and the maximum of the magnetic field close the the equatorial region (see Figure 2.3).

In Figure 2.4, it is possible to better see the magnetic field distribution along the surface of the cavity cell. The distribution of the surface magnetic field is particularly important since the dissipation in superconductors are concentrated in the high magnetic field region. Therefore, for elliptical cavities losses are concentrated in the equatorial region.

\subsection{Advantages of superconductivity}

Superconducting cavities offer many advantages compare to the normal-conducting technology. The main advantage is given by the low RF surface resistance of superconductors, which translates in Q-factors of five order of magnitude higher than the highest reachable with copper cavities. 

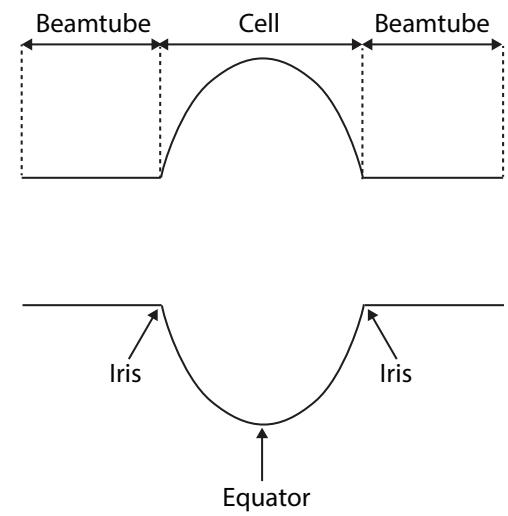

(a)

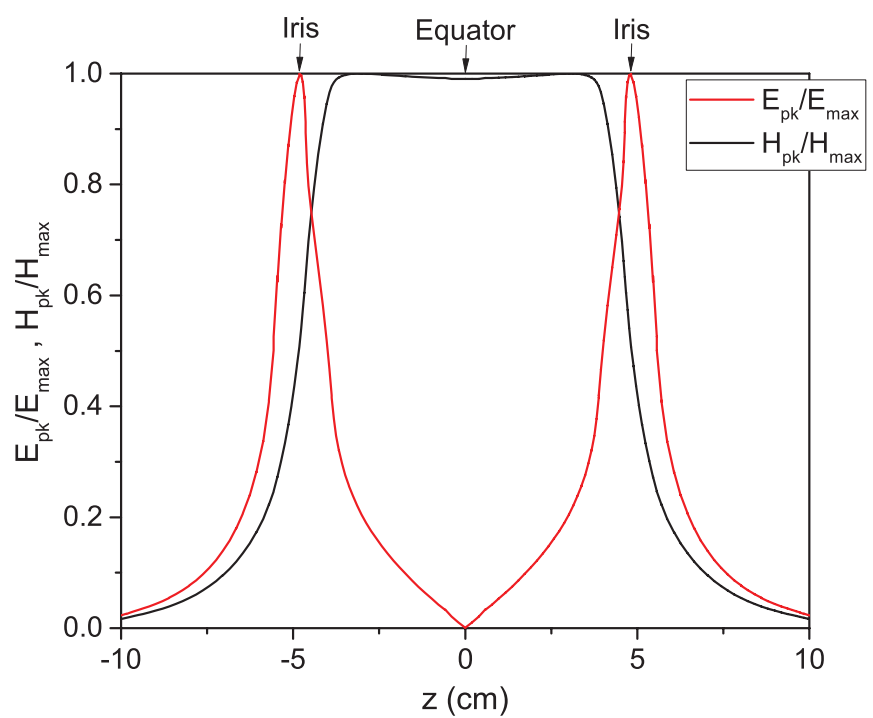

(b)

Figure 2.4. (a) Sketch of a single-cell elliptical cavity, electric and magnetic field resonates in the cavity cell, while the beam tube is needed for the passage of the beam. (b) surface electric and magnetic field distribution as a function of the distance along the beam axis, from the center of the cavity. The peak surface magnetic field is very close to the cavity equator, while the peak surface electric field is close to the cavity irises.

For continuous wave $(\mathrm{CW})$ or high duty factor accelerator the quality factor is really important in order to decrease as much as possible the power dissipated during the cavity operation. In particular, considering that the dissipated power scales with the square of the operational field, the advantage of low surface resistance value becomes of crucial importance in order to operate at large value of accelerating field.

Nowadays, typical Q-factor values of niobium $1.3 \mathrm{GHz}$ cavities at $2 \mathrm{~K}$ are larger than $10^{10}$, while copper cavities with same shape, frequency and temperature are limited to $10^{5}$. Surface resistance of normal-conductors exposed to RF field is indeed limited even at very low temperature because of the anomalous skin effect.

However, even though the Q-factors of superconducting cavities is four order of magnitude larger than the one of normal conducting cavities, the real gain in terms 
of dissipated power is lower, since the elevated power consumption due to the helium refrigeration.

In order to calculate the real gain given by the superconducting technology, a normal-conducting cavity is compared with a superconducting one which operate at the same frequency $(f=500 \mathrm{MHz})$ and accelerating field $\left(E_{a c c}=3 \mathrm{MV} / \mathrm{m}\right)$. The temperature of operation of the superconducting cavity is set at $4.2 \mathrm{~K}$, while the one of the normal-conducting cavity is $300 \mathrm{~K}$. From the definition of the shunt impedance (Equation 2.18), the dissipated power per unit of length can be defined as:

$$
\frac{P}{L}=\frac{E_{a c c}^{2}}{\frac{r_{\mathrm{a}}}{Q_{0}} Q_{0}},
$$

therefore, considering reasonable values of $r_{a} / Q$ and $Q_{0}$ (for the NC cavity: $r_{\mathrm{a}} / Q=$ $900 \Omega / \mathrm{m}, Q_{0}=2 \cdot 10^{4}$, while for the SC cavity: $\left.r_{\mathrm{a}} / Q=330 \Omega / \mathrm{m}, Q_{0}=2 \cdot 10^{9}[25]\right)$, the dissipated power per unit of length for the two case is:

$$
\frac{P}{L}=500000 \quad \mathrm{~W} / \mathrm{m} \quad, \quad \frac{P}{L}=13.6 \quad \mathrm{~W} / \mathrm{m},
$$

Now, for the normal-conducting case, it is necessary to take into account the efficiency of the RF power source which is usually around $50 \%$, increasing the required power to $1000 \mathrm{~kW} / \mathrm{m}$. For the superconducting case instead, it is necessary to take into account that also a static heat dissipation contributes to the helium bath consumption [28]. A reasonable power consumption due to static dissipation is around $1-2 \mathrm{~W} / \mathrm{m}[29,3]$. The required power also depends on the efficiency of the helium refrigeration, which is given by both the Carnot efficiency and the technical efficiency. The Carnot efficiency is given by:

$$
\eta=\frac{4.2 K}{300 K-4.2 K}=0.014,
$$

while the technical efficiency is around $0.2-0.3$ depending on the system [25]. Taking into account both the RF and the static dissipation, and considering the global refrigeration efficiency of about 0.0035 , the total required power results of about $40 \mathrm{~kW} / \mathrm{m}$. 
Therefore, the superconducting technology allows a reduction of the required power of operation of a factor of 200 when compared to the normal-conducting technology.

Since the dissipated power increases with the square of the accelerating field, it is clear that the superconducting technology becomes necessary for accelerators that operates in $\mathrm{CW}$, or with high duty factor, and that require high accelerating field. The needed of the superconducting technology is not only given by the reduction of the power consumption, but also to overcome the practical limitation for which the power dissipated in a copper cavities operating in CW can not be more than $100 \mathrm{~kW}$. This limit is imposed in order to avoid excessive temperature rising that may cause vacuum degradation, thermal expansion and so on. Because of this power limitation, copper cavities cannot operate in $\mathrm{CW}$ at accelerating field grater than $1 \mathrm{MV} / \mathrm{m}$.

In addition, for normal-conducting cavities usually the parameter $r_{a} / Q$ is maximized, decreasing the dimension of the beam hole, in order to decrease the power consumption. On the other hand, using superconducting cavities the limited power consumption open the possibility to make the beam hole much larger, increasing the stability and the quality of the beam [25]. 


\section{CHAPTER 3}

\section{INTRODUCTION TO SUPERCONDUCTIVITY}

\subsection{Overview}

In 1911 Kamerlingh Onnes observed for the first time that the electrical resistance of Mercury drops to zero when cooled to the liquid Helium temperature (4.2 $\mathrm{K})$. This phenomenon in which the material shows zero resistivity if cooled below its critical temperature $\left(T_{c}\right)$ is called superconductivity and the material superconductor.

Other than the zero resistance when DC current is applied, superconductors show also another peculiar characteristic: when they are exposed to external magnetic field, the field is completely expelled from the interior and the magnetic induction is equal to zero inside the superconductor. This perfect screening from the external field is given by super-currents that circulate in a tiny layer at the surface of the material. This behavior is called Meissner-Ochsenfeld effect [30].

The superconducting transition is defined, for type I superconductor, by three critical parameters: the critical temperature $T_{\mathrm{c}}$, the critical magnetic field $H_{\mathrm{c}}$ and the critical current density $J_{c}$. These three parameters define the critical surface which divides the super-conducting and the normal-conducting phases. A material can show superconducting properties only below the critical surface $T<T_{\mathrm{c}}, H<H_{\mathrm{c}}$ and $J<J_{\mathrm{c}}$, therefore the superconducting state can be destroyed not only increasing the temperature but also increasing the magnetic field or the current density. The temperature dependence of the critical magnetic field follows the empirical relation:

$$
H_{\mathrm{c}}(T)=H_{\mathrm{c}}(0)\left[1-\left(\frac{T}{T_{\mathrm{c}}}\right)^{2}\right] \text {. }
$$

$H_{\mathrm{c}}$ as a function of the temperature is shown in Figure 3.1. From this graph it is possible to notice that the transition actually appears at $T=T_{\mathrm{c}}$ only in absence of 


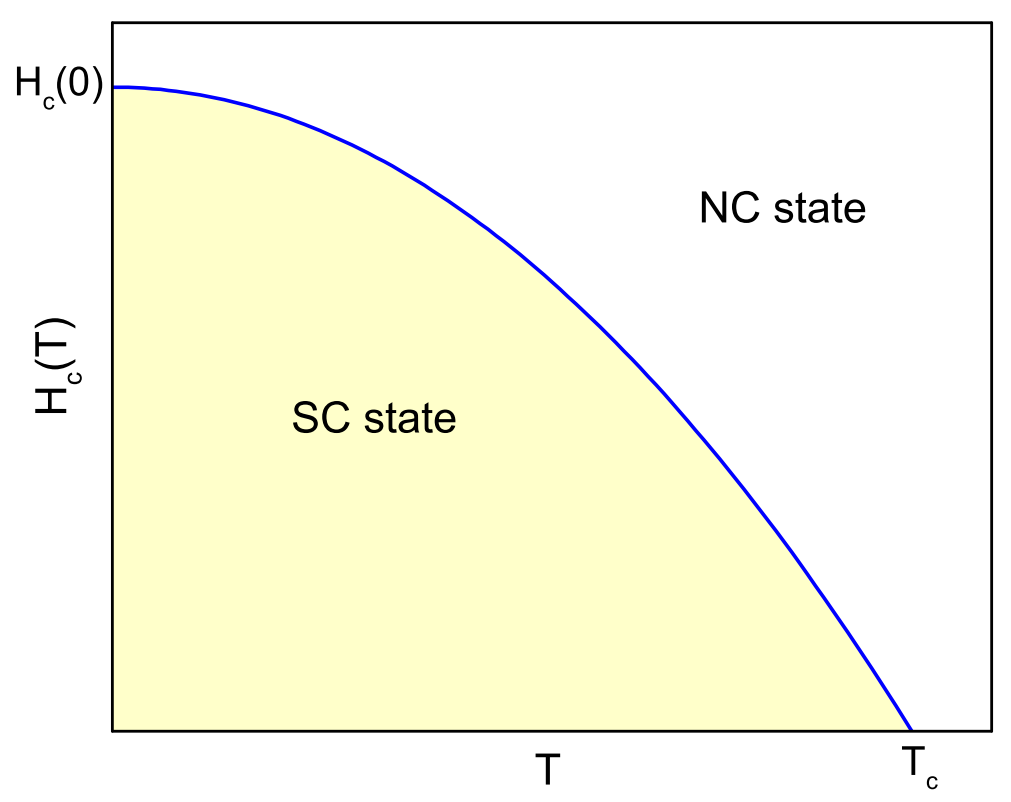

Figure 3.1. Critical magnetic field $H_{\mathrm{c}}$ as a function of the temperature $T$. For $H<H_{\mathrm{c}}$ the material is in the superconducting (SC) state, while for $H>H_{\mathrm{c}}$ it is in the normal-conducting (NC) state.

external magnetic field. If $H \neq 0$, the temperature of the superconducting transition is lowered.

The first explanation of these hallmarks of superconductivity comes from the empirical macroscopic theory of the brothers Heinz and Fritz London developed in 1935 [31]. Their theory is based on the two fluid model, developed by Gorter and Casimir [32] for which the charge carrier in the superconductor can be divided in normal-fluid and super-fluid components. The two London equations are capable to easily explain the perfect conduction and the Meissner effect, but are not capable to explain the quantum behavior of superconductors.

The first microscopic theory capable to explain the superconductivity phenomenon was developed by John Bardeen, Leon Cooper, and Robert Schrieffer in 1957 and called BCS theory. This theory is based on the formation of electron pairs, called Cooper pairs, due to the electron-phonon interaction. All the electron pairs 
must obey at the Bose statistic, so they condense in a common superconducting ground state with lower energy than the Fermi level of the normal state, creating an energy gap of size $2 \Delta$. The gap $\Delta$ depends on the electron-phonon interaction, so it is different for each materials, and depends on the temperature. As the temperature increases from 0 to $T_{\mathrm{c}}, \Delta$ slowly decrease, and it drops to zero for $T \rightarrow T_{\mathrm{c}}$. The unpaired electrons behave as normal electrons, while the paired electrons are superconducting electrons which have a long-range order defined by the coherence length $\xi_{0}[25,33]$.

Absorption of photons with energy grater than $2 \Delta$ or thermal excitations cause the depairing of the Cooper pairs, leading to a non-zero RF surface resistance, as widely studied by D. C. Mattis and J. Bardeen in 1958. This surface resistance term is called BCS surface resistance and nowadays used to analyze properties of superconductors exposed to RF field.

\subsection{Two fluid model}

In the two fluid model, the charge carriers of a superconductor can be divided in two components called normal- and super-fluid. The super-fluid component is a Bose condensate which do not carry entropy, indeed for $\mathrm{T}=0 \mathrm{~K}$ all the electrons are part of the super-fluid. The normal-fluid is instead responsible for energy losses via Joule effect, and for $T>T_{\mathrm{c}}$ all the electrons are normal-fluid. The total density of charge carries is therefore the sum between these two components, $n=n_{\mathrm{n}}+n_{\mathrm{s}}$, where $n_{\mathrm{n}}$ and $n_{\mathrm{s}}$ are the density of the normal- and the super-fluid respectively. The same is for the total current density $J=J_{\mathrm{n}}+J_{\mathrm{s}}$.

The first London equation:

$$
\frac{\partial \bar{J}_{\mathrm{s}}}{\partial t}=\frac{1}{\mu_{0} \lambda_{\mathrm{L}}^{2}} \bar{E}
$$

may be derived considering the equation of motion of the charge carriers of an ideal 
perfect conductor in which the electrons are freely accelerated. The super-current density is defined as $\bar{J}_{\mathrm{s}}=-n_{\mathrm{s}} e \bar{v}_{\mathrm{s}}$, where $\bar{v}_{\mathrm{s}}$ is the velocity of super-electrons and $e$ is the elementary charge. The London penetration depth $\lambda_{\mathrm{L}}$ results:

$$
\lambda_{\mathrm{L}}=\sqrt{\frac{m}{\mu_{0} e^{2} n_{s}}} .
$$

The first London equation is capable to explain the zero resistivity observed in superconductors exposed to direct current (DC). Indeed from Equation 3.2 it is possible to infer that when the current is constant in time, as in the case of DC, there is no electric field inside the superconductor and the conduction takes place without losses. On the other hand, in alternate current $(\mathrm{AC})$, the variation in time of the super-current density generates an electric field inside the material, involving a non-zero surface resistance.

The second London equation can be obtained by applying the Farady-Lenz equation $\bar{\nabla} \times \bar{E}=-\frac{\partial \bar{B}}{\partial t}$ to the first London equation:

$$
\bar{\nabla} \times \bar{J}=-\frac{\bar{H}}{\lambda_{\mathrm{L}}^{2}} .
$$

Applying the Ampere's Law to the second London equation, the latter may be rewritten as:

$$
\bar{\nabla}^{2} \bar{H}=\frac{1}{\lambda_{\mathrm{L}}^{2}} \bar{H} .
$$

Considering that the superconductor extends in the half-space $x>0$, and the field is applied parallel to its surface, along the z-direction, solution of this equation is:

$$
H_{z}=H_{0} e^{x / \lambda_{\mathrm{L}}}
$$

from which is clear that the magnetic field decays exponentially going deeper in the material. The London penetration depth $\lambda_{\mathrm{L}}$ is the decay constant that defines the distance at which the magnetic field reaches $1 / e$ of its value at the surface, where $e$ is the Euler's number. The penetration of the magnetic field at the surface of 
the superconductor is consequence of the presence of super-currents, that screen the superconductor from the external magnetic field.

The temperature dependence of $\lambda_{\mathrm{L}}$ is usually indicated as:

$$
\lambda_{\mathrm{L}}=\frac{\lambda_{0}}{\sqrt{1-\left(\frac{T}{T_{\mathrm{c}}}\right)^{4}}},
$$

which is an empirical formulation that was extrapolated by fitting experimental data of penetration depth as a function of temperature measured for mercury [1]. Comparing Equation 3.7 with 3.3 it is possible to notice that the temperature dependence of $\lambda_{\mathrm{L}}$ is given by the temperature variation of the density of super-electrons $n_{\mathrm{s}}$, being $m, \mu_{0}$ and $e$ constants.

The two fluid model allows to analyze the surface impedance of a superconductor. For the normal-fluid component the Drude formulation is valid, therefore $J_{\mathrm{n}}=\sigma_{\mathrm{n}} E$ and the conductivity $\sigma_{\mathrm{n}}$ is:

$$
\sigma_{\mathrm{n}}=\frac{n_{\mathrm{n}} e^{2} \tau}{m}
$$

where $n_{\mathrm{n}}$ is the density of the normal-electrons, $m$ the electron mass, $\tau$ the electron relaxation time.

The conductivity of the super-fluid can be instead calculated using the first London equation, assuming that in case of an RF current, $J_{\mathrm{s}}=J_{\mathrm{s} 0} e^{i \omega t}$. In this case the current density is a complex number:

$$
J_{\mathrm{s}}=-i \frac{1}{\omega \mu_{0} \lambda_{L}^{2}} E=-i \sigma_{\mathrm{s}} E,
$$

from which it is possible to define:

$$
\sigma_{\mathrm{s}}=\frac{n_{\mathrm{s}} e^{2}}{m \omega}
$$

The conductivity of a superconductor is therefore a complex quantity:

$$
J=\left(\sigma_{\mathrm{n}}-i \sigma_{\mathrm{s}}\right) E
$$


and the surface impedance may be defined as:

$$
Z_{\mathrm{s}}=R_{\mathrm{s}}+i X_{\mathrm{s}}=\sqrt{\frac{i \omega \mu_{0}}{\sigma_{\mathrm{n}}-i \sigma_{\mathrm{s}}}} .
$$

In the approximation $\sigma_{\mathrm{n}}<<\sigma_{\mathrm{s}}$, the real and the imaginary part of the impedance in the two fluid model are:

$$
\begin{array}{r}
R_{s}=\frac{1}{2} \sigma_{\mathrm{n}} \omega^{2} \mu_{0}^{2} \lambda_{\mathrm{L}}^{3} \\
X_{s}=\omega \mu_{0} \lambda_{\mathrm{L}},
\end{array}
$$

Where the temperature dependence of the surface resistance $R_{\mathrm{s}}$ is given by the temperature dependence of $\lambda_{\mathrm{L}}$. It is also possible to notice that $R_{\mathrm{S}}$ is proportional to the normal-state conductivity $\sigma_{\mathrm{n}}$, meaning that a good superconductor has poor normal-conducting properties. Since $\sigma_{\mathrm{n}}$ is directly proportional to the electronic mean free path $\ell$, this implies that $R_{\mathrm{s}}$ increases with $\ell$, so dirty superconductors (small $\ell$ ) should show lower RF dissipation. Important for the choosing of the cavity frequency and design is that the surface resistance has a square dependence with the frequency.

\subsection{From local to non-local electrodynamics}

In 1950 it was experimentally observed that the penetration depth of superconductors is affected by the introduction of impurities, as shown in Figure 3.2. Since this fact can not be explained using the phenomenological approach of the London theory, Pippard proposed a new non-local description capable to explain the variation of the penetration depth with the introduction of impurities. This non-local description relates the super-currents with an average of the vector potential calculated over a region around the considered point. The size of this region is defined by the coherent length $\xi$ which depends on the mean free path $\ell$. The Pippard's current density is therefore:

$$
\bar{J}(\bar{r})=-\frac{3}{4 \pi \mu_{0} \lambda^{2} \xi_{0}} \int \frac{\bar{R}\left[\bar{R} \cdot \bar{A}\left(\bar{r}^{\prime}\right)\right] e^{-R / \xi}}{R^{4}} d \bar{r}^{\prime}
$$




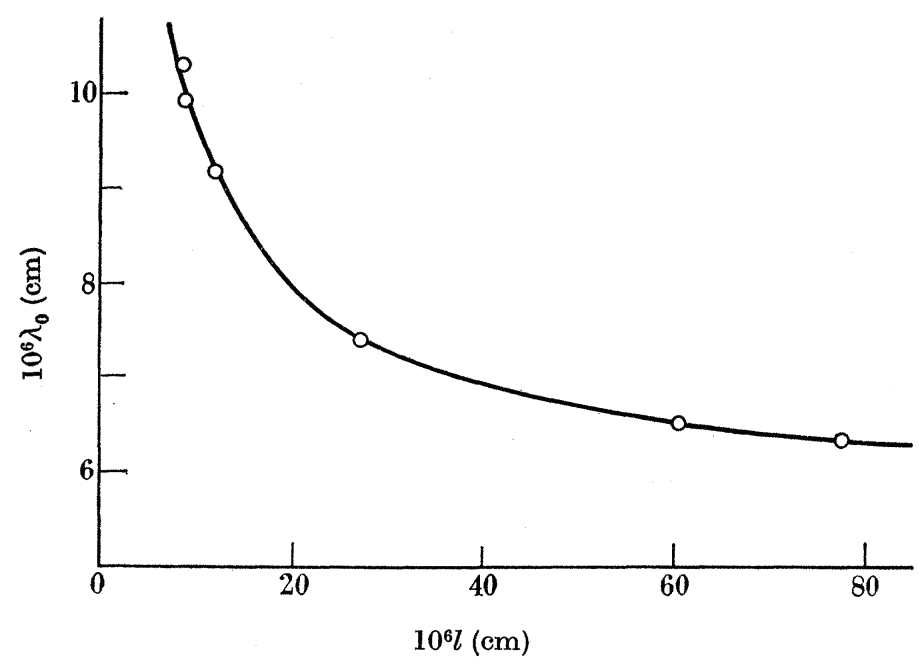

Figure 3.2. Variation of the penetration depth with the mean free path [1].

where $\bar{R}=\bar{r}-\bar{r}^{\prime}$, taking into account that the current at a point $\bar{r}$ depends on the vector potential at a point $\bar{r}^{\prime}$ within a distance $\xi_{0}$, called coherence length. Equation 3.14 is analogous to the non-local relation between current density and electric field found by Reuter and Sondheimer [34], capable to explain the anomalous skin effect in normal-metal. In Equation 3.14, $\xi$ is the parameter which depends on the purity of the material, while $\xi_{0}$ is a constant with dimension of a length. These parameters are related by the equation:

$$
\frac{1}{\xi}=\frac{1}{\xi_{0}}+\frac{1}{\ell}
$$

where can be noticed that in case of a very clean metal: $\ell \rightarrow \infty$ and $\xi \rightarrow \xi_{0}$. Therefore $\xi_{0}$ can be considered as the coherence length of the pure metal.

Using the Maxwell equation $\nabla^{2} \bar{A}=-\mu_{0} \bar{J}$, Equation 3.14 can be solved for the penetration depth $\lambda$, which is defined as:

$$
\lambda=\frac{1}{H_{\mathrm{y}}(0)} \int_{0}^{\infty} H_{\mathrm{y}} d z
$$

The result can be simplified by looking at the two limits: 
- $\xi<<\lambda$ :

$$
\lambda=\lambda_{\mathrm{L}} \sqrt{\frac{\xi_{0}}{\xi}}=\lambda_{\mathrm{L}} \sqrt{1+\frac{\xi_{0}}{\ell}},
$$

- $\xi>>\lambda$ :

$$
\lambda_{\infty}=\frac{3^{1 / 6}\left(4 \pi \lambda_{\mathrm{L}}^{2} \xi_{0}\right)^{1 / 3}}{2 \pi^{2 / 3}} .
$$

From these equations it is possible to notice that for $\xi<<\lambda, \lambda$ depends on $\xi^{-1 / 2}$, meaning that the penetration depth increases as the mean free path decreases, while for $\xi>>\lambda, \lambda$ is independent on $\xi$, in agreement with the experimental result shown in Figure 3.2. These values of $\lambda$ represent the real penetration depth that defines the magnetic field decay inside the superconductor. The Pippard's $\lambda$ is therefore sometimes called effective penetration depth in order to distinguish it from the London's definition.

\subsection{The Ginsburg and Landau theory}

During approximately the same period, in 1950, Vitaly Lazarevich Ginzburg and Lev Landau proposed a theory capable to explain the superconducting transition phase, called Ginzburg-Landau (GL) theory [35]. The GL theory is based on Landau's theory of second-order phase transition in which a complex pseudo-wavefunction $\psi$ was introduced as order parameter. This wavefunction describes the local density of super-electrons: $n_{\mathrm{s}}=|\psi(r)|^{2}$.

The theory is then based on the expansion in series of the free energy density $f$ for small values of $\psi$, i.e. for $T \rightarrow T_{\mathrm{c}}$ :

$$
f=f_{n}+\alpha|\psi|^{2}+\frac{\beta}{2}|\psi|^{4}+\frac{1}{2 m^{*}}\left|\left(\frac{\hbar}{i} \nabla-\frac{e^{*}}{c} \bar{A}\right) \psi\right|^{2}+\frac{h^{2}}{8 \pi},
$$

where $f_{\mathrm{n}}$ is the free energy density in the normal-conducting phase, $\alpha$ and $\beta$ are the expansion coefficients, $\hbar$ is the reduced Planck constant and the quantities $e^{*}$ and 
$m^{*}$ are the effective charge and mass of the current carriers respectively and $\bar{A}$ is the vector potential.

The constant $\alpha$ changes sign at $T=T_{\mathrm{c}}$. For $\alpha>0$ the minimum of the free energy density occurs at $|\psi|^{2}=0$ which correspond to the normal state with $T>T_{\mathrm{c}}$. On the other hand, for $\alpha<0$, the minimum occurs at $|\psi|^{2}>0$, defying the superconducting state for $T<T_{\mathrm{c}}$. At transition $\alpha=0$ therefore $\beta>0$ is a necessary condition in order to define a minimum of the free energy density [36].

Using a variation principle, the minimum of Equation 3.19 was calculated with respect to the spatial variations of $\psi(r)$ and $A(r)$, finding the two GL equations. From these equations two important parameters are defined: the temperature-dependent coherence length $\xi_{\mathrm{GL}}$, and the penetration depth $\lambda_{\mathrm{GL}}$. The GL coherent length $\xi_{\mathrm{GL}}$ represent the distance over which the order parameter can naturally vary. Even though in pure superconductors at $T<<T_{\mathrm{c}}, \xi_{\mathrm{GL}}$ approaches the Pippard coherent length $\xi_{0}$, the two parameters are distinct quantities. The GL penetration depth $\lambda_{\mathrm{GL}}$ has instead the same meaning of the London penetration depth [33].

Since $\xi_{\mathrm{GL}}(T)$ and $\lambda_{\mathrm{GL}}(T)$ have the same temperature dependence, their ratio define a useful dimensionless parameter, called GL parameter:

$$
\kappa=\frac{\lambda}{\xi}
$$

This parameter is particularly useful to distinguish between different classes of superconductors: Type I $(\kappa<1 / \sqrt{2})$ and Type II $(\kappa>1 / \sqrt{2})$, depending on the sign of their surface energy.

In presence of external field $H_{\mathrm{e}}$, the boundary energy between the superconducting and the normal-conducting phase is:

$$
\frac{\mu_{0}}{2}\left(\xi_{0} H_{c}^{2}-\lambda_{\mathrm{L}} H_{\mathrm{e}}^{2}\right)
$$


Therefore $\xi_{0}$ and $\lambda_{\mathrm{L}}$ determines the response of a superconductor exposed to external magnetic field. When $\xi_{0}>\lambda_{\mathrm{L}}$ the surface energy is positive, while for $\xi_{0}<\lambda_{\mathrm{L}}$ the surface energy is negative. This means that, when $\lambda>\xi$, the total energy is minimized in presence of superconducting/normal-conducting interfaces [25].

As shown in Figure 3.1, Type-I superconductors show a discontinuous breakdown of the superconducting state, when the external magnetic field is increased above the critical field $H_{\mathrm{c}}$.

Abrikosov in 1957 showed that for type II superconductors instead, it is necessary to defined two different critical fields: the lower critical field $H_{\mathrm{c} 1}$ and the upper critical field $H_{\mathrm{c} 2}$. For $H<H_{\mathrm{c} 1}$ the material is completely in the superconducting state, which is called now Meissner state, while for $H>H_{\mathrm{c} 2}$ the material is completely in the normal-conducting state (see Figure 3.3a). When the external field is in between these two critical fields, the material is in the so-called mixed state, in which flux can penetrate in the superconductor forming a regular array of flux tubes. Each flux tube carries a magnetic flux quantum, called fluxoid:

$$
\Phi_{0}=\frac{h}{2 e}=2.07 \cdot 10^{-15} W b
$$

and a vortex of supercurrent concentrates the flux toward its center, screening the superconductor from the flux tube (see Figure 3.3b). Such magnetic flux structures are called vortices.

When vortices are present in the superconductor, and a static transport current $\bar{J}$ is established, the vortices are moved by the Lorentz force $\bar{J} \times \bar{\phi}_{0}$ causing dissipation inside the material. This situation can be controlled by the presence of defects in the material that can pin the flux, minimizing its free energy. So the static current $\bar{J}$ can flow without dissipation as long as the Lorentz force is lower than the pinning force $[33]$. 


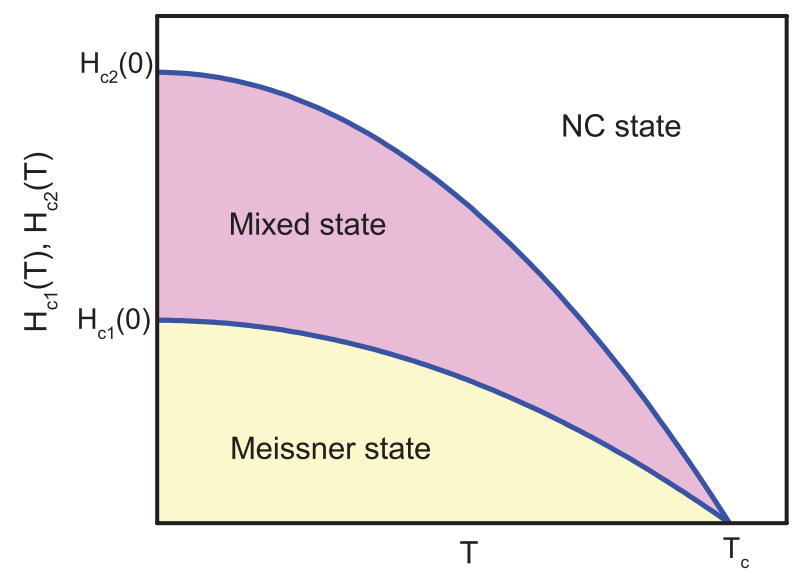

(a)

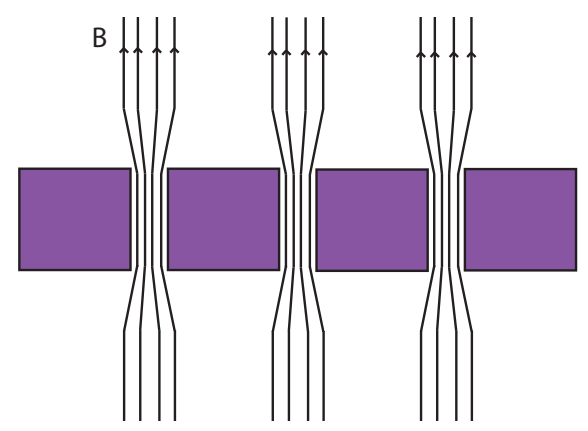

(b)

Figure 3.3. (a) Lower $\left(H_{\mathrm{c} 1}\right)$ and upper $\left(H_{\mathrm{c} 2}\right)$ critical magnetic fields as a function of the temperature for a type II superconductor. For $H<H_{\mathrm{c} 1}$ the superconductor is in the Meissner state, while for $H_{\mathrm{c} 1}<H<H_{\mathrm{c} 2}$ it is in the mixed state. The normal-conducting state is instead defined for $H>H_{\mathrm{c} 2}$. (b) Sketch of a side view of the magnetic flux structures present in the mixed state.

\subsection{The BCS theory}

In 1957 Bardeen, Cooper and Schrieffer published a microscopic theory of superconductivity [37]. They demonstrated that superconductivity is consequence of an attractive interaction existing between electrons that allows the formation of electron pairs called Cooper pairs. This attractive interaction is due to exchange of a lattice phonon between electrons. A bound state forms as a result of this interaction, with energy lower than the Fermi energy of the normal-conductor.

The idea of an electron-phonon interaction was suggested by Fröhlich in 1950 and experimentally confirmed with the discovery of the isotope effect, i.e. the dependence of the critical temperature of a superconductor on the mass of the isotope composing the material.

This interaction may be thought as due to an electron that passing through the lattice, polarizes the medium by attracting positive ions. In turn, the excess 
of positive charge attracts a second electron, resulting in an attractive interaction between electrons. If this interaction is greater than the repulsive Coulomb interaction a Cooper pair is formed. Important is also that, in order to form Cooper pairs, electrons must have both opposite momentum and spin.

When Cooper pairs are formed they condense in a common superconducting ground state with lower energy than the Fermi level, creating an energy gap of size $2 \Delta$.

The Cooper pairs have the following characteristics:

- The size of a Cooper pair is $\sim \xi_{0}=\hbar v_{\mathrm{F}} / k T_{\mathrm{c}}$, which is much larger than the interparticle distance, meaning that Cooper pairs overlap with each other.

- Cooper pairs represent a Bose condensate, so they all have same quantum state and same energy.

- When current flows in the superconductor, each Cooper pair acquires a momentum which is the same for all the pairs.

- The total momentum of a Cooper pair is constant.

- The minimum energy needed to broke a Cooper pair is equal to the energy gap $E_{\mathrm{g}}=2 \Delta$. The breaking of a pair generates two quasi-particle excitations.

The formation of an energy gap between the superconducting ground state and the normal-conducting ground state, was already proven experimentally by Corak et al. [38], from measurements of specific heat of superconductors. Such measurements showed that at $T<<T_{\mathrm{c}}$ the specific heat has an exponential dependence with the temperature, implying the presence of an energy gap [33]. 
The energy gap at $T=0 \mathrm{~K}, \Delta(0)$, is defined as:

$$
\Delta(0) \simeq 2 \hbar \omega_{\mathrm{c}} e^{-1 / N(0) V}
$$

where $\omega_{\mathrm{c}}$ is the cut-off frequency of pair attraction and is of the order of magnitude of the Debay frequency $\omega_{\mathrm{D}}, N(0)$ is the density of states at the Fermi level for electrons of one spin orientation and $V$ in the potential of the attractive interaction. Since $N(0)$ and $V$ are material dependent parameters, the energy gap $\Delta$ is a characteristic parameter that varies between different materials. Important to take into account is that Equation 3.23 is an approximate results obtained with the weak-coupling approximation $N(0) V<<1$.

The energy gap depends also on the temperature: as the temperature increases from 0 to $T_{\mathrm{c}}, \Delta$ slowly decrease, and it drops rapidly to zero for $T \rightarrow T_{\mathrm{c}}$. A useful approximated formula capable to describe this behavior is:

$$
\frac{\Delta(T)}{\Delta_{0}}=\sqrt{\cos \left(\frac{\pi t^{2}}{2}\right)} .
$$

In the framework of the BCS theory, the critical temperature is defined as the temperature at which $\Delta(T) \rightarrow 0$ and results to be:

$$
k T_{c}=1.13 \hbar \omega_{\mathrm{c}} e^{-1 / N(0) V}
$$

Comparing this with Equation 3.23, it is possible to notice that the energy gap $\Delta(0)$ is of the same order of magnitude of $k T_{\mathrm{c}}$ :

$$
\frac{\Delta(0)}{k T_{\mathrm{c}}} \simeq 1.764
$$

this value was found to be reasonable from different experiments, however it varies from superconductor to superconductor and it is usually between 1.5 and 2.2 [33].

As previously mentioned, Cooper pairs can be broken with energy grater than $2 \Delta$, generating quasi-particle excitations and, therefore, dissipation in the superconductor. The electrodynamic properties of superconductors were studied by Bardeen, 
Cooper and Schriefeer using a similar non-local approach of Pippard, by defining that the current density is a function of the vector potential $\bar{A}$ :

$$
\bar{J}(\bar{r})=-\frac{3}{4 \pi \mu_{0} \lambda^{2} \xi_{0}} \int \frac{\bar{R}[\bar{R} \cdot \bar{A}(\bar{r})] J(R, T)}{R^{4}} d \bar{r},
$$

where $R$ is the distance between scattering centers and the kernel $J(R, T)$, at $T=0$ $\mathrm{K}$, approaches at the Pippard coefficient $e^{-R / \xi_{0}}$. A more convenient expression is given by the Fourier transform of $\bar{J}(\bar{r})$ :

$$
\bar{J}(\bar{q})=-\frac{c}{4 \pi} K(q) \bar{a}(\bar{q})
$$

where $K(q)$ is a function that in the limit $\bar{q}=0$ approaches to the inverse of the square of the London penetration depth: $K(0, T)=1 / \lambda^{2}(T)$. The penetration depth, defined as in Equation 3.16, can be calculated from the current density [37]. In case of specular reflection, the penetration depth results:

$$
\lambda=\frac{2}{\pi} \int_{0}^{\infty} \frac{1}{q^{2}+K(q)} d q
$$

while in case of random scattering:

$$
\lambda=\frac{\pi}{\int_{0}^{\infty} \ln \left[1+\frac{K(q)}{q^{2}}\right] d q}
$$

In the approximation of $\bar{q}=0$, which is the case of $\xi_{0}<<\lambda$, and for $T / T_{\mathrm{c}}>0.8$, the temperature dependence of the penetration depth approaches at the empirical formula of Equation 3.7, while for $T / T_{\mathrm{c}}<0.5$ [39]:

$$
\frac{\lambda(T)-\lambda(0)}{\lambda(0)}=\sqrt{\frac{\pi \Delta}{2 k T}} e^{-\frac{\Delta}{k T}} .
$$

In 1958, Mattis and Bardeen refines the BCS treatment of the electrodynamic properties of superconductors, deriving a general quanto-mechanic expression for the kernel $I(\omega, R, T)$ in the BCS expression for the current density [40]:

$$
\bar{J}(\bar{r}, t)=\sum_{\omega} \frac{e^{2} N(0) v_{0}}{2 \pi^{2} \hbar c} \int \frac{\bar{R}\left[\bar{R} \cdot \overline{A_{\omega}}(\bar{r})\right] I(\omega, R, T) e^{-R / \ell}}{R^{4}} d \bar{r} .
$$


This extension is valid for field of arbitrary frequencies, while the BCS current density of Equation 3.27 is valid only for low frequency.

In order to derive an expression for the conductivity of superconductors, Mattis and Bardeen introduced a complex conductivity for the superconducting state, $\sigma_{\mathrm{s}}=$ $\sigma_{1}-i \sigma_{2}$, as previously defined by Glover and Tinkham [41]. This conductivity depends on the kernel $I(\omega, R, T)$ and, in extreme anomalous limit, $\lambda<<\xi_{0}$, for which it is possible to set $R=0$, it is defined as:

$$
\sigma_{\mathrm{s}}=\sigma_{\mathrm{n}} \frac{I(\omega, 0, T)}{-\pi i \hbar \omega}
$$

From this equation it is possible to define $\sigma_{1}$ and $\sigma_{2}$ :

$$
\begin{gathered}
\frac{\sigma_{1}}{\sigma_{\mathrm{n}}}=\frac{2}{\hbar \omega} \int_{\Delta}^{\infty}[f(E)-f(E+\hbar \omega)] g(E) d E \\
+\frac{1}{\hbar \omega} \int_{\Delta-\hbar \omega}^{-\Delta}[1-2 f(E+\hbar \omega)] g(E) d E, \\
\frac{\sigma_{2}}{\sigma_{\mathrm{n}}}=\frac{1}{\hbar \omega} \int_{\Delta-\hbar \omega,-\Delta}^{\Delta} \frac{[1-2 f(E+\hbar \omega)]\left(E^{2}+\Delta^{2}+\hbar \omega E\right)}{\left(\Delta-E^{2}\right)^{1 / 2}\left[(E+\hbar \omega)^{2}-\Delta^{2}\right]^{1 / 2}} d E,
\end{gathered}
$$

these two expressions are usually called Mattis and Bardeen integrals and they have to be resolved numerically. The term $g(E)$ is defined as:

$$
g(E)=\frac{\left(E^{2}+\Delta^{2}+\hbar \omega E\right)}{\left(E^{2}-\Delta^{2}\right)^{1 / 2}\left[(E+\hbar \omega)^{2}-\Delta^{2}\right]^{1 / 2}},
$$

and $f$ is the Fermi-Dirac distribution function.

The real part of the conductivity $\sigma_{1}$ is the term from which dissipation arises in the superconductor, and from Equation 3.34 it is possible to distinguish that there are two mechanisms that can cause dissipation. The first integral of $\sigma_{1}$ takes into account thermal excitation of quasi-particle, while the second integral takes into account absorption of photons with energy greater than the energy gap, indeed this term does not appear unless $\hbar \omega>2 \Delta$. The imaginary part of the conductivity $\sigma_{1}$ takes instead account generation of Cooper pairs. 
Knowing the two contributions at the conductivity $\sigma_{1}$ and $\sigma_{2}$, it is possible to calculate the surface impedance which, in the extreme anomalous limit, is defined as:

$$
Z_{\mathrm{s}}=Z_{\mathrm{n}}\left(\frac{\sigma_{1}-i \sigma_{2}}{\sigma_{\mathrm{n}}}\right)^{-1 / 3}
$$

The real part of the surface impedance defines the surface resistance $R_{\mathrm{s}}$.

Considering the case of superconducting cavities, dissipations are only due to thermal excitation of quasi-particle, since $\hbar \omega<2 \Delta$. Therefore only the first integral of Equation 3.34 has to be taken into account. This integral can be solved analitically in the approximation $T<<T_{\mathrm{c}}$. Using this approximation and $T>>\hbar \omega / 2 k T$, the surface resistance results [42]:

$$
R_{\mathrm{BCS}}=\frac{\mu_{0}^{2} \omega^{2} \lambda^{3} \sigma_{\mathrm{n}} \Delta}{k T} \ln \left(\frac{2.246 k T}{\hbar \omega}\right) e^{-\frac{\Delta}{k T}} .
$$

This equation shows that the surface resistance:

- exponentially decreases with the temperature,

- depends on the square of the angular frequency $\omega$,

- is directly proportional to the conductivity of the normal-conducting state $\sigma_{\mathrm{n}}$,

- depends on the material mean free path by the dependence with $\lambda^{3} \sigma_{\mathrm{n}}$, in agreement with the two fluid model result (Equation 3.13).

The dependence of $\lambda$ on the mean free path cannot be easily seen from Equations 3.29 and 3.30, therefore in order to look at the dependence of $R_{\mathrm{BCS}}$ with the mean free path it is convenient to take into account the Pippard's definition shown in Equation 3.17. The conductivity $\sigma_{\mathrm{n}}$ is instead defined by the Drude formula (Equation 3.8). Therefore:

$$
R_{\mathrm{BCS}} \propto \lambda^{3} \sigma_{\mathrm{n}} \propto \ell\left(1+\frac{\xi_{0}}{\ell}\right)^{3 / 2}
$$



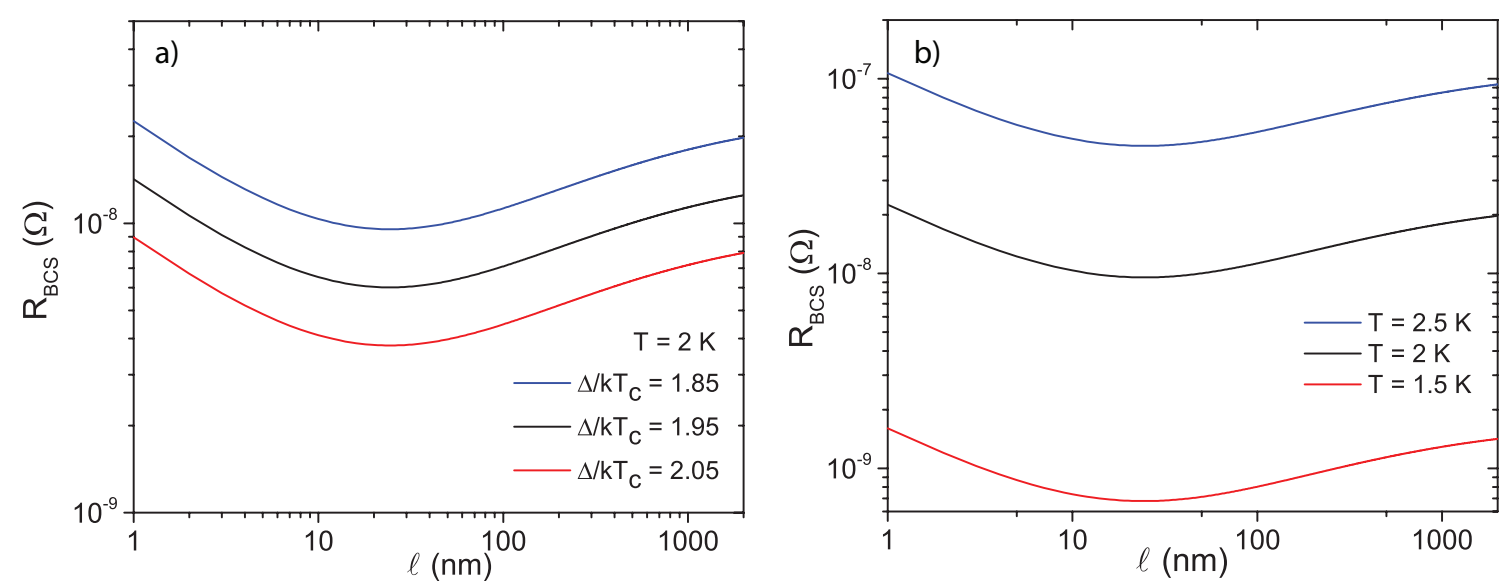

Figure 3.4. BCS surface resistance as a function of the mean free path for different values of reduced energy gap $\Delta / k T_{\mathrm{c}}(\mathrm{a})$, and for different values of temperature $T$ (b). The curves are calculated using the program SRIMP [2].

this relation has a minimum at $\ell=\xi / 2$, meaning that the surface resistance is minimized when the mean free path is around $\xi / 2$.

The surface resistance as a function of mean free path calculated using the Mattis and Bardeen theory is shown in Figure 3.4. The graphs are generated using the Halbritter code called SRIMP [2]. In Figure 3.4 (a), curves of $R_{\mathrm{BCS}}$ as a function of $\ell$ are shown for different values of the reduced energy gap $\Delta / k T_{\mathrm{c}}$, keeping all the other parameters fixed. It is clear that increasing $\Delta / k T_{\mathrm{c}}$ the resistance decreases. In Figure 3.4 (b), curves of $R_{\mathrm{BCS}}$ versus $\ell$ are shown for different temperatures. The dependence of the BCS surface resistance with the mean free path was first verified by Flécher [43].

Also the exponential dependence of the surface resistance on the temperature was experimentally verified. However, comparing the theoretical behaviour with experimental data, see Figure 3.5, it is possible to notice that the experimental data can be fitted by the BCS theory up to a certain temperature, below that temperature the surface resistance assets to a constant value.

Therefore, the complete expression of the surface resistance must take into ac- 


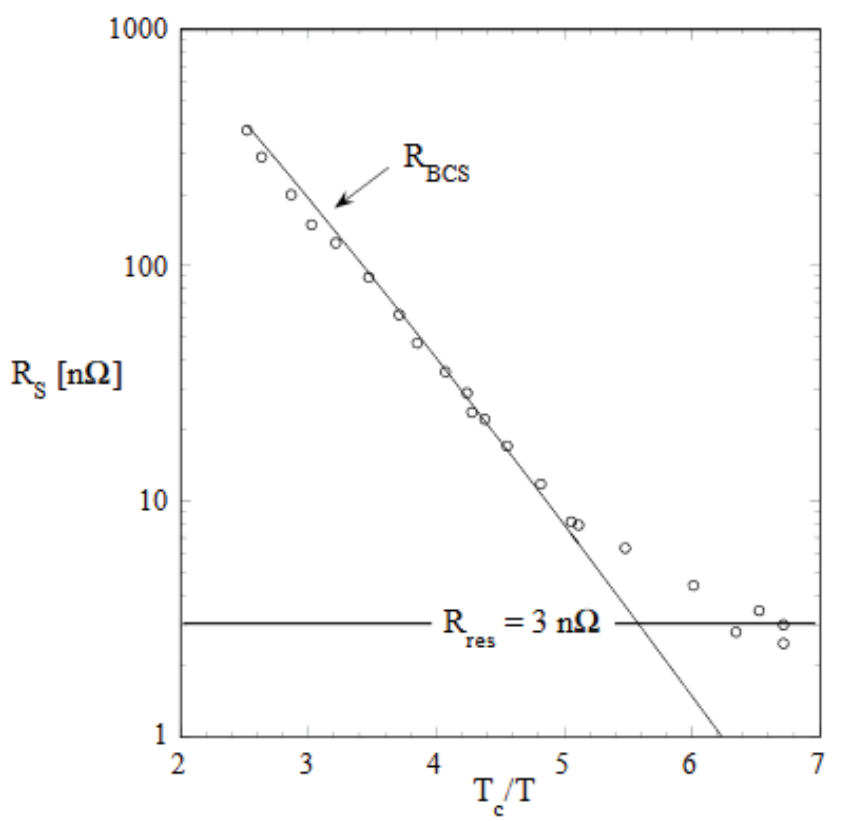

Figure 3.5. Comparison between experimental and theoretical data of the surface resistance as a function of the temperature [3].

count not only the temperature-dependent term derived using the Mattis and Bardeen theory, $R_{\mathrm{BCS}}$, but also a temperature-independent term which is called residual resistance, $R_{0}$ :

$$
R_{\mathrm{s}}=R_{\mathrm{BCS}}+R_{0}
$$

The residual resistance term is not fully understood yet, possible causes may be: presence of defects within the superconductor penetration depth region, non-ideality of the surface, magnetic flux trapped in the superconductor, presence of hydrides or oxidized layer on the surface or within grain boundaries, residuals from chemical or mechanical surface treatment, welding imperfections, and so on [25].

The BCS surface resistance, Equation 3.38, is usually approximate using the following relation:

$$
R_{\mathrm{BCS}}=\frac{A}{T} \omega^{2} e^{-\Delta / k T},
$$

where $A$ is a constant which depends on the following material parameters: London 
penetration depth $\lambda_{\mathrm{L}}$, coherence length $\xi_{0}$, Fermi velocity $v_{F}$, mean free path $l$. This relation is valid for $T<T_{\mathrm{c}} / 2$ [25]. 


\section{CHAPTER 4 \\ RF MEASUREMENTS AND CAVITY PROCESSING}

\subsection{Power balance in a resonator}

Because of the very narrow resonant peak, characteristic of superconducting cavities, the quality factor cannot be measured directly with a network analyzed but has to be measured through a power balance between the forwarded, reflected, transmitted and dissipated powers. This kind on RF measurement is described in the following section.

The fundamental resonant mode is excited in the cavity by using a RF source. The power from the RF source is carried inside the cavity using an input probe called input coupler, while the transmitted power probe picks up the power transmitted through the cavity. A typical configuration is shown in Figure 4.1.

There are different methods used to couple the field in or out of the cavity, the most common are: the electric coupling using a coaxial antenna, the magneticcoupling using a loop at the end of a coaxial transmission line, or a wave-guide connected through a hole in the cavity. The configuration used for the experiment described in this thesis work, consists on electric coupling with two antennas acting as input and output couplers.

These antennas are cylindrical conductors that penetrate the cavity generating a coaxial cable configuration, in which the antenna is the inner conductor and the cavity is the external one. The overlap between the cavity field and the field propagating in the cut-off region from the input coupler defines the coupling strength. The coupling strength increases exponentially by inserting the coupler in the cavity. Usually the coupling strength is minimized for the transmitted probe.

In order to understand the power dissipation of the whole system, it is useful 


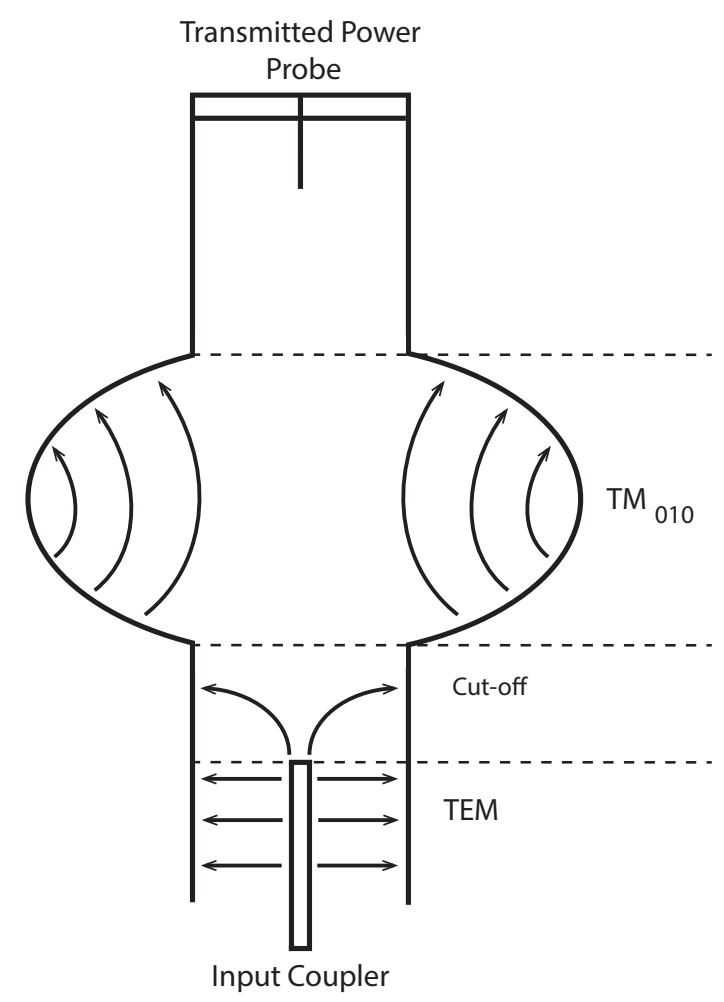

Figure 4.1. Scheme of a single-cell cavity with the two antennas, input coupler and transmitted power probe, needed for the RF measurement.

to begin analyzing the situation just after that the RF source is switched off. A fraction of power is dissipated in the cavity walls, while another fraction leaks out from both input and output couplers. The total dissipated power $P_{\text {tot }}$ is given by the sum of all these contribution:

$$
P_{\mathrm{tot}}=P_{\mathrm{c}}+P_{\mathrm{e}}+P_{\mathrm{t}}
$$

where $P_{\mathrm{c}}$ is the power dissipated in the cavity walls, $P_{\mathrm{e}}$ is the power leaking back through the input coupler and $P_{\mathrm{t}}$ is the power leaking forward to the transmitted probe. The total dissipated power is related to the unloaded quality factor $Q_{\mathrm{L}}$, which is the quality factor of the entire system composed by cavity, input coupler and output coupler:

$$
Q_{\mathrm{L}}=\frac{\omega_{0} U}{P_{\mathrm{tot}}}
$$

As seen in previous section, the energy stored in the cavity $U$ exponentially decay 
with a time constant $\tau=Q_{\mathrm{L}} / \omega_{0}$ :

$$
U=U_{0} e^{-\frac{\omega_{0} t}{Q_{\mathrm{L}}}}
$$

meaning that the loaded quality factor can be calculated by measuring the time decay of the stored energy and knowing the cavity resonance frequency.

The intrinsic quality factor of the cavity is now indicated with $Q_{0}$ and takes into account only dissipation coming from the cavity walls. The external quality factors $Q_{1}$ and $Q_{2}$ take instead into account the power dissipated in the two antennas, $P_{\mathrm{e}}$ and $P_{\mathrm{t}}$ respectively:

$$
Q_{1}=\frac{\omega_{0} U}{P_{\mathrm{e}}} \quad, \quad Q_{2}=\frac{\omega_{0} U}{P_{\mathrm{t}}}
$$

The coupling strength between the cavity and the couplers can be quantified as:

$$
\beta_{1}=\frac{Q_{0}}{Q_{\mathrm{e}}}=\frac{P_{\mathrm{e}}}{P_{\mathrm{c}}} \quad, \quad \beta_{2}=\frac{Q_{0}}{Q_{\mathrm{t}}}=\frac{P_{\mathrm{t}}}{P_{\mathrm{c}}}
$$

therefore, the stronger is the coupling strength, the larger is the power dissipated in the coupler compared to the power dissipated in the cavity walls. Note that the external quality factors depend only on the geometry of both cavity and coupler, while the coupling strengths depend also on the intrinsic quality factor.

The intrinsic quality factor $Q_{0}$ can be calculated knowing the loaded quality factor and the coupling strengths:

$$
\frac{1}{Q_{\mathrm{L}}}=\frac{1}{Q_{0}}+\frac{1}{Q_{1}}+\frac{1}{Q_{2}}=\frac{1}{Q_{0}}\left(1+\beta_{1}+\beta_{2}\right)
$$

Usually the transmitted probe is weakly coupled with the cavity, $\beta_{2}<<1$, so that the dissipation in that coupler are negligible and the Q-loaded may be easily defined as:

$$
\frac{1}{Q_{\mathrm{L}}}=\frac{1}{Q_{0}}\left(1+\beta_{1}\right)
$$


Starting from an empty cavity, when the RF power reaches the cavity at first there is a transient behavior in which the stored energy increases with the time until it reaches its equilibrium value $U$. Let us now take into account a situation in which the cavity is in steady state, so it had already reached the equilibrium, and is driven exactly on resonance.

At this point it is necessary to take into account also the forward power $P_{\mathrm{f}}$, which is the power forwarded to the cavity from the RF generator, and the reflected power $P_{\mathrm{r}}$, which is the subtraction between the forward power and the power that leaks in the cavity. Under the previous assumption, $P_{\mathrm{r}}$ is defined as:

$$
P_{\mathrm{r}}=P_{\mathrm{f}}|\Gamma|^{2}=P_{\mathrm{f}}\left|\frac{\beta_{1}-1}{\beta_{1}+1}\right|^{2},
$$

where $\Gamma$ is the reflection coefficient which depends strongly on $\beta_{1}$.

In particular, if $\beta_{1}=1$, then $\Gamma=0$ and there is no reflected power, meaning that all the forwarded power enters in the cavity. This situation in which cavity and coupler are perfectly matched is called critical coupling. Sometimes the input coupler is a movable antenna so that it can be adjusted to reach this condition. The system is instead undercoupled when $\beta_{1}<1$, and overcoupled when $\beta_{1}>1$.

From the conservation of energy, the power that actually flows in the cavity results:

$$
P_{\text {in }}=P_{\mathrm{f}}\left(1-|\Gamma|^{2}\right)
$$

therefore, when the system is critically coupled $P_{\text {in }}$ is maximum and equal to the forward power.

Knowing $P_{\mathrm{f}}$ and $P_{\mathrm{r}}$ it is possible to calculate the coupling strength $\beta_{1}$ using the following equation:

$$
\beta_{1}=\frac{1 \pm \sqrt{P_{\mathrm{r}} / P_{\mathrm{f}}}}{1 \mp \sqrt{P_{\mathrm{r}} / P_{\mathrm{f}}}}
$$


which is calculated by solving Equation 4.8 for $\beta_{1}$. The upper sign is used when the system is overcoupled, while the lower sign when it is undercoupled.

\subsection{RF measurements}

Once the cavity has been properly treated, it has to be RF tested in order to verify its performance. The cavity is therefore assembled with two fixed antenna, the input and output couplers, in a clean room in order to avoid the introduction of contaminants inside the cavity. Once the cavity is vacuum sealed and evacuated, it is mounted on a RF stand that holds the cavity inside the cryostat. The cryostat can be at this point filled with liquid helium. Once the cavity is soaked liquid helium $(4.2 \mathrm{~K})$, the temperature is decreased reaching, usually, 2 or $1.5 \mathrm{~K}$ by pumping on the helium bath.

Once the cavity reached the desired temperature, the RF test can start.

Because of the narrow resonant peak of superconducting cavities, the cavity is kept on resonance by means of a phase-looked-loop. The phase-looked-loop is composed of a voltage-controlled-oscillator (VCO), a mixer and a phase shifter. The VCO generates the initial RF signal at the cavity resonance frequency, which is amplified and fed into the cavity. Using a bidirectional coupler, the forward signal can be measured with a power meter before entering in the cavity, and another power meter measure the reflected signal. Similarly, also the transmitted signal is measured with a power meter. Part of the transmitted signal is then fed into the RF mixer, together with part of the signal generated by the VCO. The mixer then generates a voltage that is proportional to the phase difference between the two signals and this voltage is used to correct the frequency of the signal generated by the VCO.

A picture of part of the RF system present at the vertical test facility at

Fermilab is shown in Figure 4.2 (a). In Figure 4.2 (b) is shown a picture of the three 


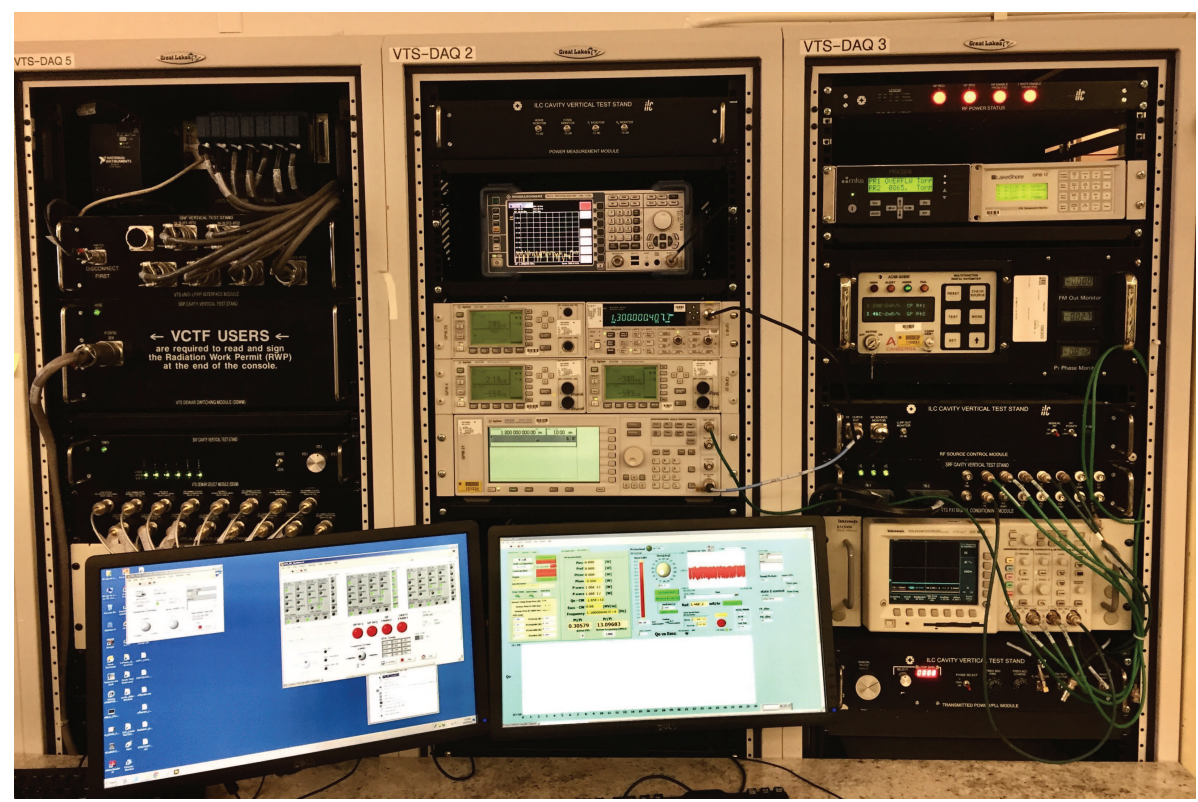

(a)

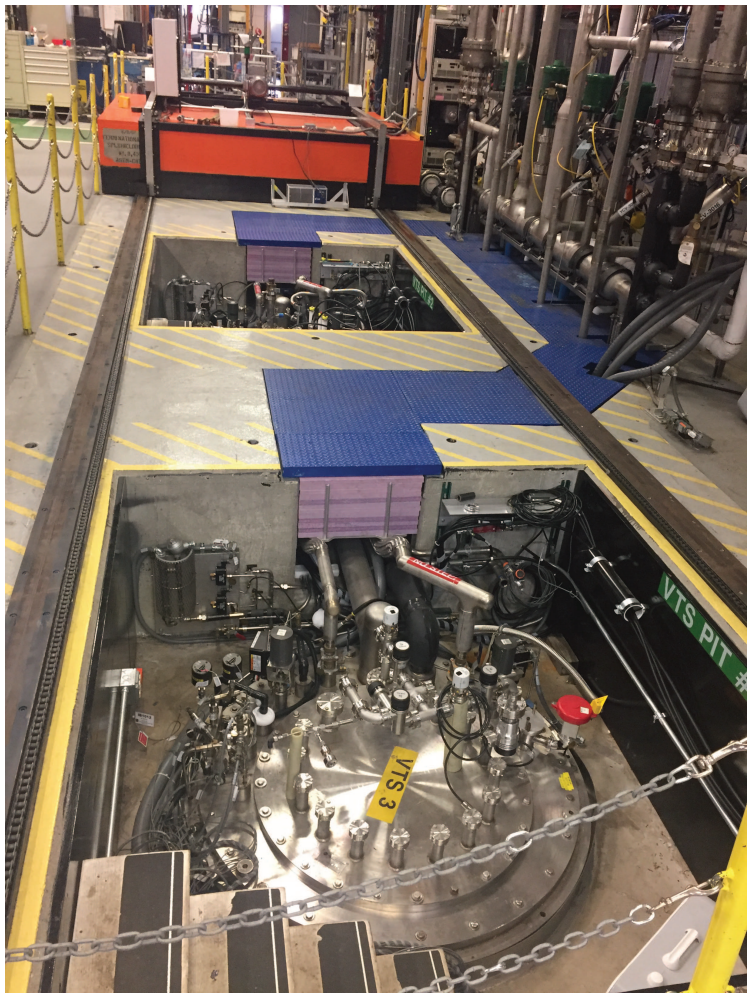

(b)

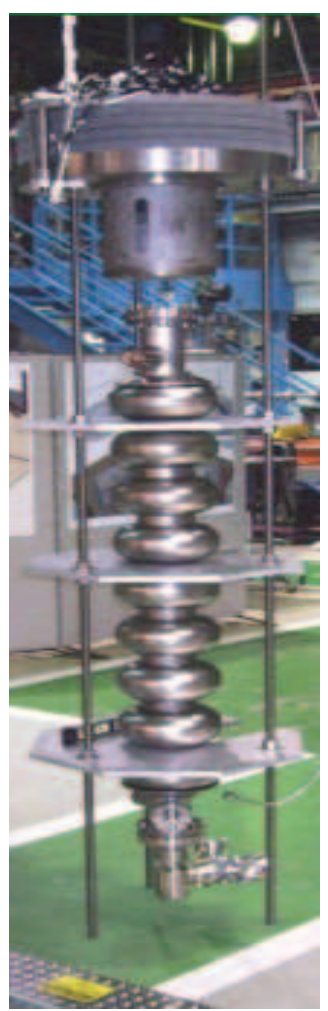

(c)

Figure 4.2. Pictures of: (a) the RF measurement system, (b) the three cryostat used for the cavities vertical test and (c) a 9-cells cavity mounted on the RF stand. This instrumentation is part of the vertical test facility at Fermilab. 
cryostat of the facility, while in (c) is shown a picture of a 9-cells cavity hanged on the RF stand.

The RF measurement can be divided in three main step: RF cable calibration, decay measurement and CW measurement.

4.2.1 Cable calibration. Since the forward, reflected and transmitted powers are not directly measured at the input and transmitted couplers, the RF cable calibration is needed in order to take into account dissipations due to all the RF components that are in between couplers and power meters, such as: cables, directional couplers, attenuators and so on.

In Figure 4.3, a scheme of the circuit of the RF system, useful to understand the different steps of the cable calibration is shown.

The labels A, B, C, D, E, F, G indicates the points at which the power is measured during the calibration process. The purpose of this calibration step is to determine the power attenuation between:

- DF: in order to estimate what is the real power forwarded in the cavity compared to the power level read by the incident power meter

- DG: in order to estimate what is the real power reflected back from the cavity compared to the power level read by the reflected power meter

- $\mathrm{AC}$ : in order to estimate what is the real power transmitted by the cavity compared to the power level read by the transmitted power meter

The power attenuation measured between AC, DF and DG defines the respective coefficient $C_{\mathrm{f}}, C_{\mathrm{r}}$ and $C_{\mathrm{t}}$ needed to convert the power measured by the power 


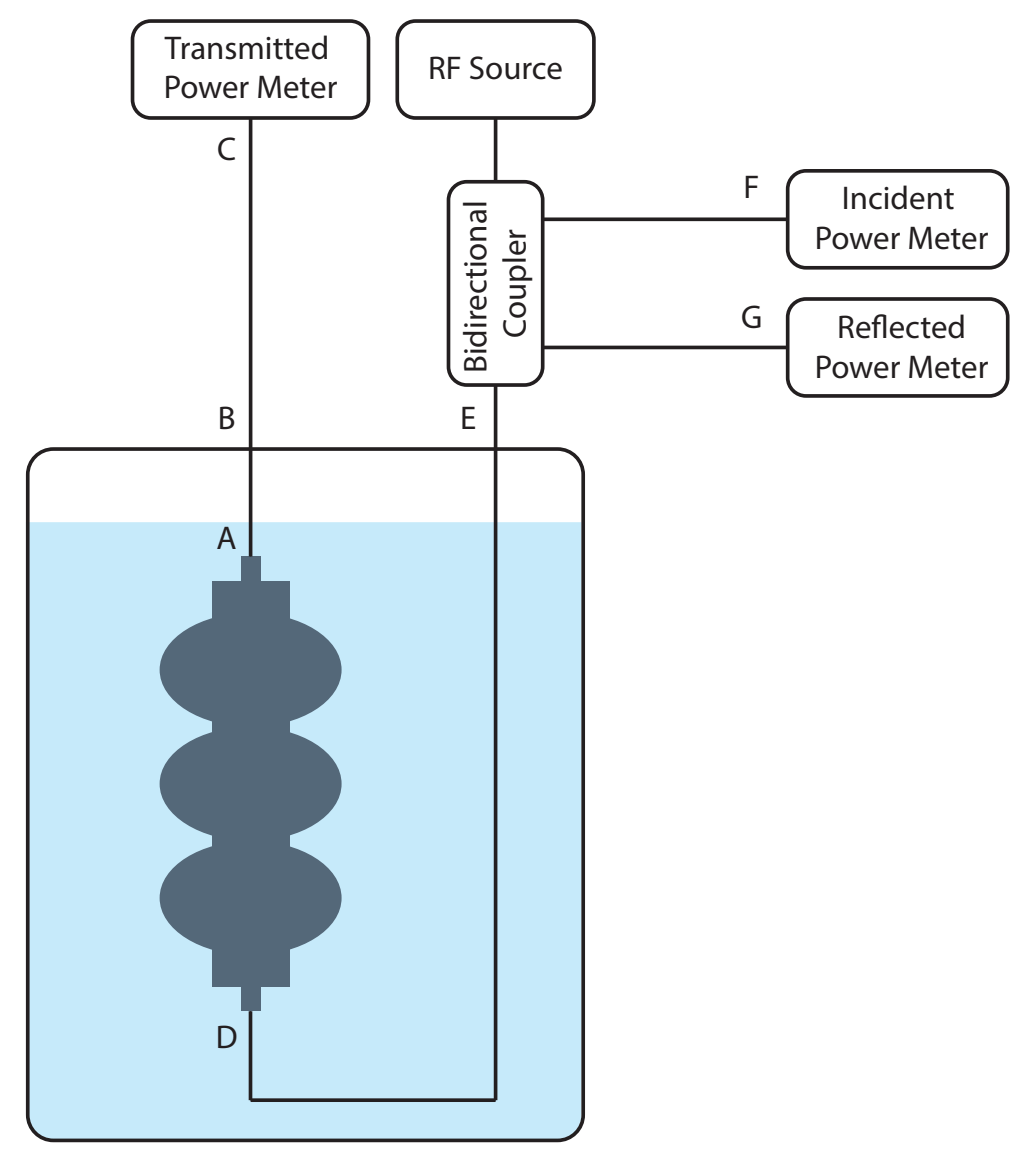

Figure 4.3. Scheme of the vertical test RF calibration system.

meters in the real forward, reflected and transmitted powers:

$$
P_{\mathrm{f}, \mathrm{r}, \mathrm{t}}^{\text {cavity }}=C_{\mathrm{f}, \mathrm{r}, \mathrm{t}} \cdot P_{\mathrm{f}, \mathrm{r}, \mathrm{t}}^{\text {measured }}
$$

where the coefficients $C_{\mathrm{f}}, C_{\mathrm{r}}$ and $C_{\mathrm{t}}$ are measured as follow:

$$
\begin{gathered}
C_{\mathrm{f}}=10^{\left(-C_{\mathrm{FE}}-C_{\mathrm{ED}}\right)}, \\
C_{\mathrm{r}}=10^{\left(C_{\mathrm{GE}}+C_{\mathrm{ED}}\right)}, \\
C_{\mathrm{t}}=10^{\left(C_{\mathrm{CB}}+C_{\mathrm{BA}}\right)},
\end{gathered}
$$

where $C_{\mathrm{FE}}$ is the power attenuation between the incident power meter and the forward connection at the top plate of the cryostat, $C_{\mathrm{GE}}$ and $C_{\mathrm{CB}}$ are the analogous for the reflected and transmitted line respectively, $C_{\mathrm{ED}}$ is the power attenuation of the cold part of both the forward and reflected line, from the connection at the cryostat flange 


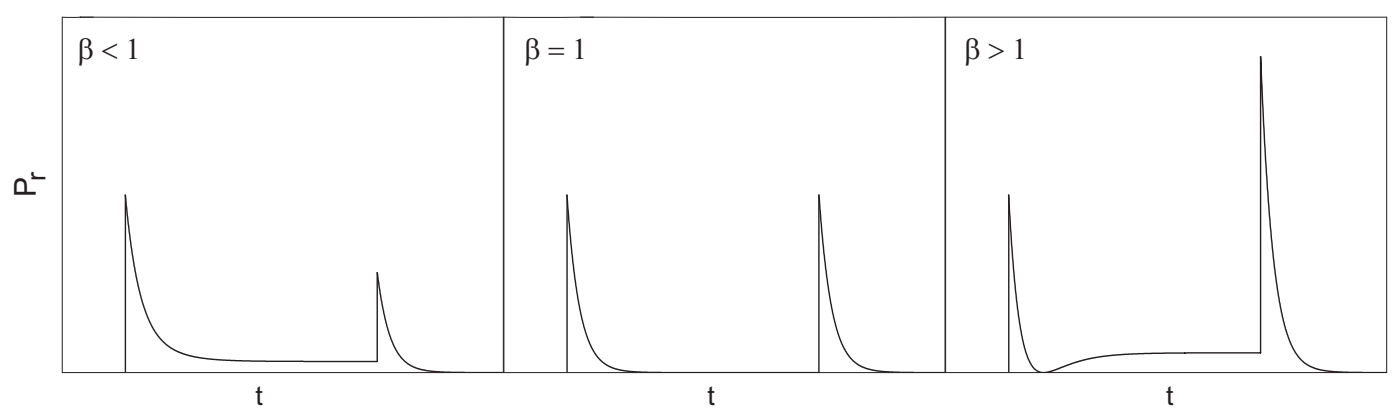

Figure 4.4. Reflected power response in pulse mode, as a function of time, for different coupling regime.

to the forward coupler, and $C_{\mathrm{ED}}$ is the power attenuation of the cold part of the transmitted line, as shown in Figure 4.3.

4.2.2 Decay measurement. The decay measurement step is needed in order to measure the external quality factor of the transmitted power probe $Q_{2}$.

Once the cavity is excited at its resonance frequency $f_{0}$, the forward, reflected and transmitted powers are recorded. From the power balance it is possible to calculated the power dissipated in the cavity walls:

$$
P_{\mathrm{c}}=P_{\mathrm{f}}-P_{\mathrm{r}}-P_{\mathrm{t}}
$$

Knowing the forward and the reflected powers it is possible to calculate the coupling strength neglecting the presence of the output coupler using Equation 4.10, where the different signs have to be chosen depending on whether the system is over- or under-coupled, as explained in the previous paragraph. An easy way to understand that consists on looking at the shape of the reflected power when a rectangular drive pulse is fed into the cavity, as shown in Figure 4.4 .

Once $\beta_{1}$ is known, it is possible to calculate the real input coupling strength $\beta$ as [27]:

$$
\beta=\beta_{1}\left(1+\beta_{2}\right)
$$

where $\beta_{2}$ is easily calculated as $\beta_{2}=P_{\mathrm{t}} / P_{\mathrm{c}}$. 
At this point the power is turned off. In this way it is possible to calculate the decay constant $\tau_{\mathrm{L}}$ by looking at the decay profile of the transmitted or reflected power level. The loaded quality factor is therefore estimated as $Q_{\mathrm{L}}=2 \pi f_{0} \tau_{\mathrm{L}}$. The loaded quality factor is then converted in intrinsic quality factor:

$$
Q_{0}=Q_{\mathrm{L}}\left(1+\beta_{1}+\beta_{2}\right)
$$

and the transmitted probe quality factor calculated as:

$$
Q_{2}=\frac{Q_{0}}{\beta_{2}}
$$

After this calibration step, the first point of the curve is measured. $Q_{0}$ is obtained from Equation 4.15, while the accelerating field is calculated as:

$$
E_{\mathrm{acc}}=\sqrt{\frac{U}{k}},
$$

where $U=Q_{0} P_{\mathrm{c}} / \omega_{0}$ and $k$ is a constant parameter that depends on the cavity geometry and is usually calculated using simulation codes.

The knowledge of the value of $Q_{2}$ allows to measure the other points of the curve with an easier CW measurement.

4.2.3 CW measurement. The CW measurement is the real measurement of the intrinsic Q-factor versus accelerating field $E_{\text {acc }}$ and it is performed from CW measurements of the power levels, using the parameter $Q_{2}$ previously determined.

The intrinsic Q-factor is now calculated as:

$$
Q_{0}=\frac{Q_{2} P_{\mathrm{t}}}{P_{\mathrm{c}}}
$$

and the accelerating field as:

$$
E_{\mathrm{acc}}=\sqrt{Q_{2} P_{\mathrm{t}}\left(\frac{r / Q}{L}\right)},
$$




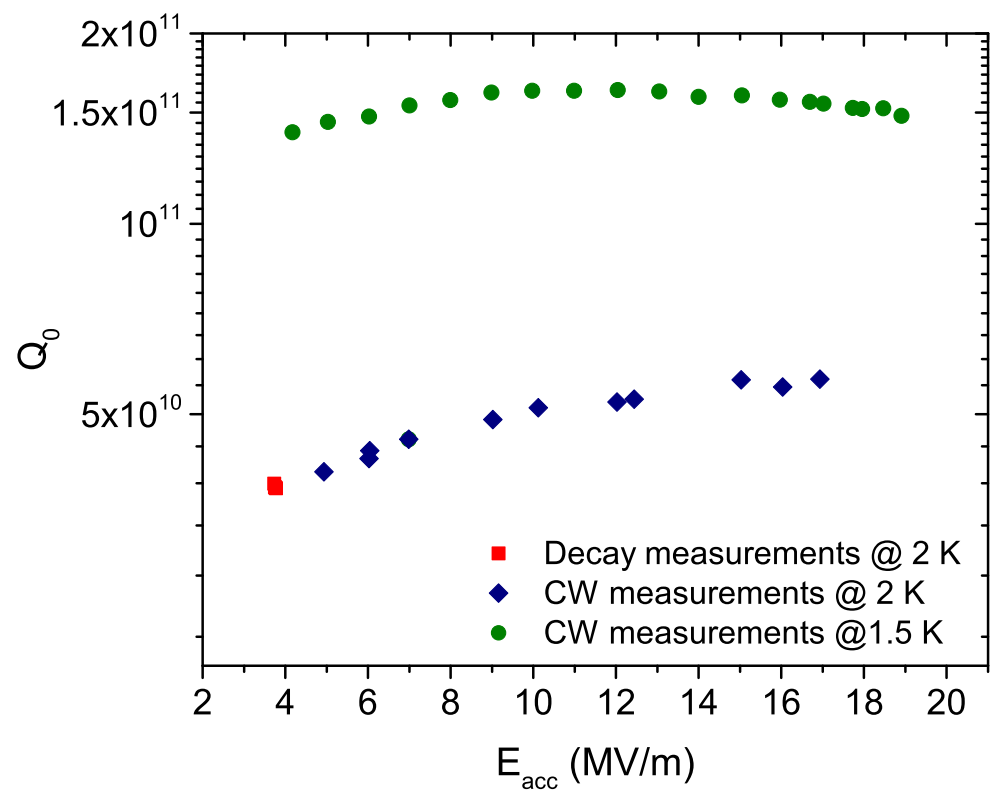

Figure 4.5. Example of Q-factor versus accelerating field curves acquired at $2 \mathrm{~K}$ and $1.5 \mathrm{~K}$. The red points are acquired in decay mode while all the other points are acquired in $\mathrm{CW}$ mode.

where $r / Q$ is the geometrical shunt impedance per unith of length and $L$ the effective length of the cavity.

\subsection{Q-factor versus accelerating field curves}

Example of two $Q_{0}$ versus $E_{\text {acc }}$ curves acquired at $2 \mathrm{~K}$ and at $1.5 \mathrm{~K}$ are shown in Figure 4.5. The points acquired during the transmitted power calibration step are highlighted in red.

This step is usually done at $2 \mathrm{~K}$ because the input coupler is a fixed antenna designed in order to be as close as possible to to the critically coupled condition at 2 K. In this way the error of both the calculation of $Q_{2}$ and all the RF measurement is minimized at $2 \mathrm{~K}$ [44]. Since $Q_{2}$ depends only on the coupler and cavity geometry, its value does not change with the temperature, therefore the transmitted power calibration does not need to be repeated again after changing the temperature.

The measurement errors on the Q-factors and accelerating field values depend 
on the coupling strength with the input antenna. The more the measurement is done in a critically coupled condition, the lower is the measurement uncertainty. The antennas adopted in the RF measurements at Fermilab usually introduce a relative error of about $10 \%$ [44].

The maximum accelerating field shown in the graph usually represents the maximum accelerating field reachable before the quench of the superconducting state.

The quench is due to a point on the cavity surface that suddenly turns normalconducting, causing a dramatic increasing of the surface resistance and of the dissipation. This, in turn, causes the spreading of the normal-conducting area that increases consuming all the stored energy in the resonator.

Quench in superconducting cavities can be due to several reasons. The most common quench scenarios are the thermal breakdown and the magnetic enhancement. The thermal breakdown appears if defects, in which RF losses are higher than the other area of the cavity, are present. In this case the anomalous temperature increasing as a function of the accelerating field at the defect can be detected until the temperature around it becomes larger than $T_{\mathrm{c}}$. At this point the whole area turns normal-conducting causing the quench. Quench via magnetic enhancement happens instead if the RF magnetic field at the cavity surface exceeds the local critical field $H_{\mathrm{c} 1}$. This situation may be facilitated by the presence of irregularity and asperities at the cavity surface, from which the local magnetic field is enhanced $[25,45]$.

Sometimes quench is instead due to different phenomena such as multipacting or field emission. In these peculiar case the quench is not a direct consequence but it can happen in the most severe cases [25].

After the quench, the cavity may shows higher surface resistance values. During the opening of the normal-conducting area some flux can be trapped locally, 
causing additional dissipation [46]. For this reason sometimes RF measurements are interrupted at a certain $E_{\text {acc }}$ value, in order to not quench the cavity. This administrative limit is applied in particular when the curve $Q$ versus $E_{\text {acc }}$ has to be acquired at different temperature. In this case the cavity is not quenched until the last curve is acquired, so that the measurement is not affected by additional dissipation.

\subsection{Niobium cavities fabrication and preparation}

The performance of niobium accelerating cavities may be really different depending on the surface treatment. In particular, since at $2 \mathrm{~K}$ the RF field penetrates for just some tens of nanometers, the properties of the material in this thin surface layer determines all the RF superconducting properties of the cavity.

During the years, the fabrication process of niobium cavities has been optimized in order to obtain very clean niobium cavities with very smooth surface. The main common steps of the fabrication are here reported.

After the extraction the niobium ore is purified in an electron-beam melting furnace. During this process impurities evaporates out the niobium and are pumped away. Then a final annealing step is performed for recristallization. Depending on the vacuum level of the furnace, temperature and time of the recristallization process, the niobium reaches very high level of purity, measured in Residual Resistivity Ratio (RRR). The RRR is the ratio between the resistivity measured at room temperature $\rho(300 \mathrm{~K})$, and the residual resistivity $\rho_{\mathrm{i}}$, usually measured just before the superconducting transition:

$$
R R R=\frac{\rho(300 K)}{\rho_{\mathrm{i}}} .
$$

Since at very low temperature the phonons contribution at the resistivity becomes negligible, $\rho_{\mathrm{i}}$ is only given by the scattering with the impurities. The RRR indicates indeed the purity level of the material, and it is therefore directly propor- 
tional to the mean free path. Typical values of RRR of niobium sheets used for cavities are around $R R R=300-500$.

In order to turn the niobium sheets in the elliptical shape typical of high- $\beta$ niobium cavities, deep drawing is usually used to form half-cells. The half-cells are then electron beam welded together in a vacuum chamber to create the elliptical cell. The beam tubes are electron beam welded at the irises of the cell.

After the fabrication process, the inner surface of the cavity is electro-polished (EP) in order to remove the first $100-150 \mu m$ from the surface, that may have been contaminated during previous processes. The EP treatment is done using the niobium cavity as the positive electrode (anode) while an alluminum cage inserted inside the cavity is the cathode. The electrolyte is made with about 9 volumes of sulphuric acid $\left(\mathrm{H}_{2} \mathrm{SO}_{4}\right)$ and 1 volume of hydrofluoric acid $(\mathrm{HF})$. The EP is capable to give a very smooth niobium surface and it is not sensitive to defects, grains orientations and so on $[47]$.

Cavities are then usually further processed in order to improve performance. Typically cavities are baked at $800{ }^{\circ} \mathrm{C}$ in a ultra high vacuum (UHV) furnace for 3 hours. This baking process is needed to degas the hydrogen that may have been adsorbed in the niobium during the EP step. Cavities measured without this kind of baking may show a peculiar Q-factor decreasing as a function of the accelerating field, that starts from low field level, as shown in Figure 4.6 [4]. This behavior is called Qdisease and appears when cavities are parked for long time at temperatures between 100 and $150 \mathrm{~K}$ during the cooldown. At these temperatures, if the concentration of hydrogen is large enough (greater than $\simeq 100-200 \mathrm{ppm}$ ), niobium hydrides can nucleate and grow. Such $N b_{x} H_{y}$ compounds are superconducting only at very low fields, at $5 \mathrm{MV} / \mathrm{m}$ losses are already very large [45]. 


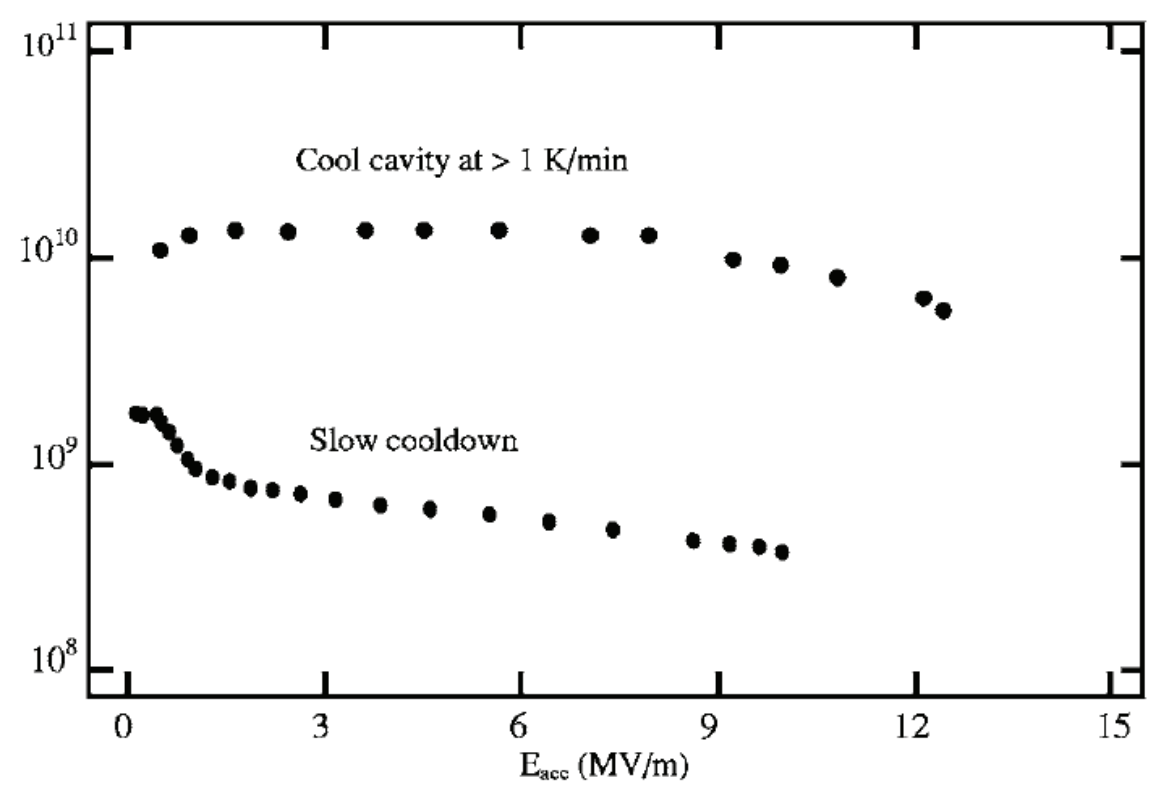

Figure 4.6. Q-factor degradation due to Q-disease after a slow cooldown [4].

In order to avoid that contaminants from the furnace jeopardize the cavity performance, after the $800{ }^{\circ} \mathrm{C}$ baking a further EP can be done to remove the first $\simeq$ $50 \mu \mathrm{m}$ from the inner surface of the cavity. Alternatively, niobium caps are used to cover the cavity beam tubes and avoid that contaminants from the furnace reach the inner surface of the cavity [48].

Cavities prepared in this way are called EP cavities and a typical $Q$ versus $E_{a c c}$ curve at $2 \mathrm{~K}$ is shown in Figure 4.7. Characteristic feature of these cavities is the presence of high-field Q-slope (HFQS) above $25 \mathrm{MV} / \mathrm{m}$. The HFQS is a really important issue for superconducting cavities since limits the usable accelerating field at around $25 \mathrm{MV} / \mathrm{m}$.

The presence of niobium hydrides is considered to be a possible mechanism responsible for the HFQS. Differently from the hydrides responsible for the Q-disease, this time this compounds must be very small. Under this hypothesis, these nanohydrides are superconducting via proximity effect, until the surface magnetic field 


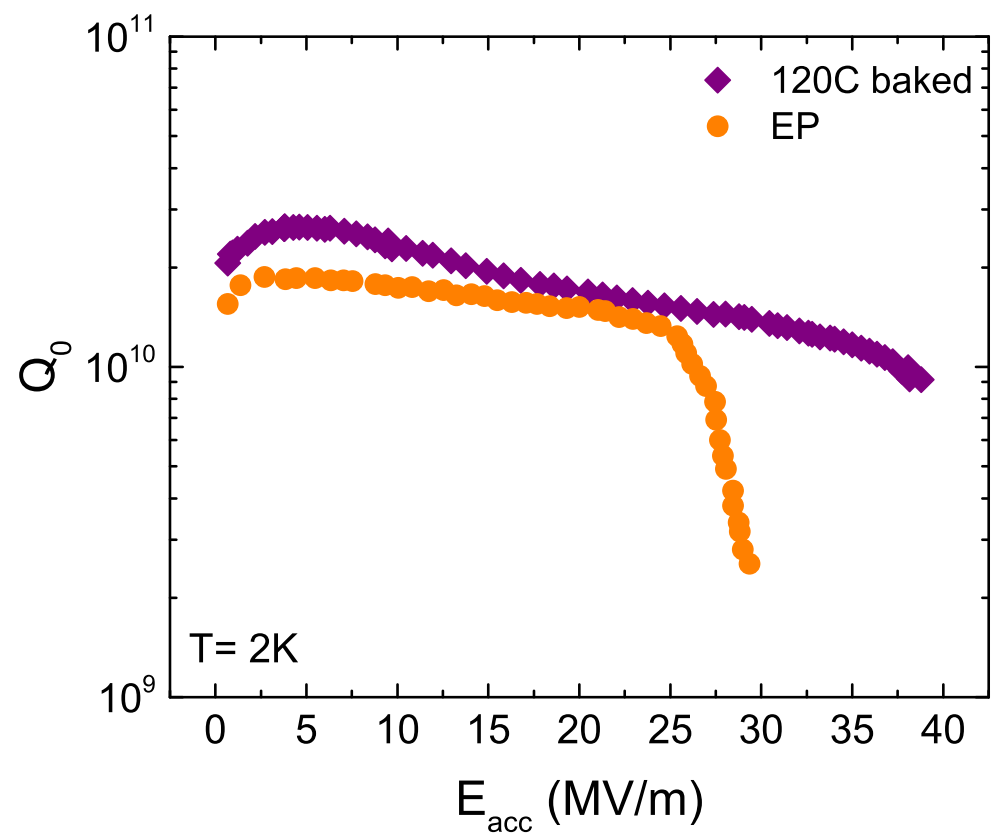

Figure 4.7. Q-factor versus accelerating field curves measured at $2 \mathrm{~K}$ for an EP and a $120^{\circ} \mathrm{C}$ baked cavity.

reaches their breakdown field $H_{\mathrm{b}}$. When $H \geq H_{\mathrm{b}}$ they start to behave as normalconducting defects, increasing the dissipation [49].

Another chemical treatment that can be used as an alternative to the EP, or as an additional chemical treatment, is the buffered chemical polishing (BCP) which is a chemical etching made with 1 volume of hydrofluoric acid (HF), 1 volume of nitric acid $\left(\mathrm{HNO}_{3}\right)$ and 2 parts of phosphoric acid $\left(\mathrm{H}_{3} \mathrm{PO}_{4}\right)$. This chemical treatment is really easy to perform and the etching rate is fast, however $\mathrm{BCP}$ tends to etch preferentially grain boundaries and the etch rate depends on the grain orientation. The surface after the BCP is therefore more rough than after EP [47]. Because of this, EP is usually preferred as final polishing treatment.

After the chemical treatments the cavity is further cleaned with the highpressure rinsing (HPR), in which ultra-pure water is sprayed at high pressure (about 100 bar) inside the cavity. The water jet strikes all the inner surface of the cavity, 
removing microscopic contaminants from it. After the cavity is completely dried inside a Class 10 or 100 clean room, the cavity is assembled with the antennas needed for the RF test and then is evacuated in order to reach a good vacuum level $(p \simeq$ $10^{-7}$ mbar $)$. The evacuation is done slowly in order to avoid turbulent flow that may bring contaminants from the vacuum system to the cavity. At this point the cavity is ready to be connected to the RF stand and inserted in the cryostat for the RF measurement.

It was found that the HFQS may be cured by further baking the EP, or BCP, cavity at $120{ }^{\circ} \mathrm{C}$ for 48 hours (see Figure 4.7). Such baking treatment is done after that the cavity has already been assembled with the antennas for the RF test.

There are experimental evidences that show that during the $120{ }^{\circ} \mathrm{C}$ baking process some oxygen from the oxidized surface layer diffuses inside the cavity for a depth not larger than $\simeq 100 \mathrm{~nm}[50]$. However, the mechanism that seems to prevent the formation of nano-hydrides is the diffusion of vacancies that are favorable to bound the hydrogen preventing the nano-hydrides precipitation [51]. In terms of Qfactors at low and medium field, the introduction of these impurities on the surface decreases a little bit the BCS surface resistance but, on the other hand, increases the values of residual resistance of a couple of nanohoms $[50,52]$.

The discovery of the $120^{\circ} \mathrm{C}$ baking treatment represented a large improvement for the SRF field, since $120{ }^{\circ} \mathrm{C}$ baked cavities are able to reach accelerating fields larger than $35 \mathrm{MV} / \mathrm{m}$. Such achievement is particularly important for accelerators that need high field level such as the International Linear Collider (ILC). The $120^{\circ} \mathrm{C}$ baking represents indeed the standard treatment of niobium cavities for the ILC. Since considered a reliable SRF technology, the $120{ }^{\circ} \mathrm{C}$ baking has also been implemented as the cavities treatment for the cryomodule production of the European X-ray Free Electron Laser (XFEL). 


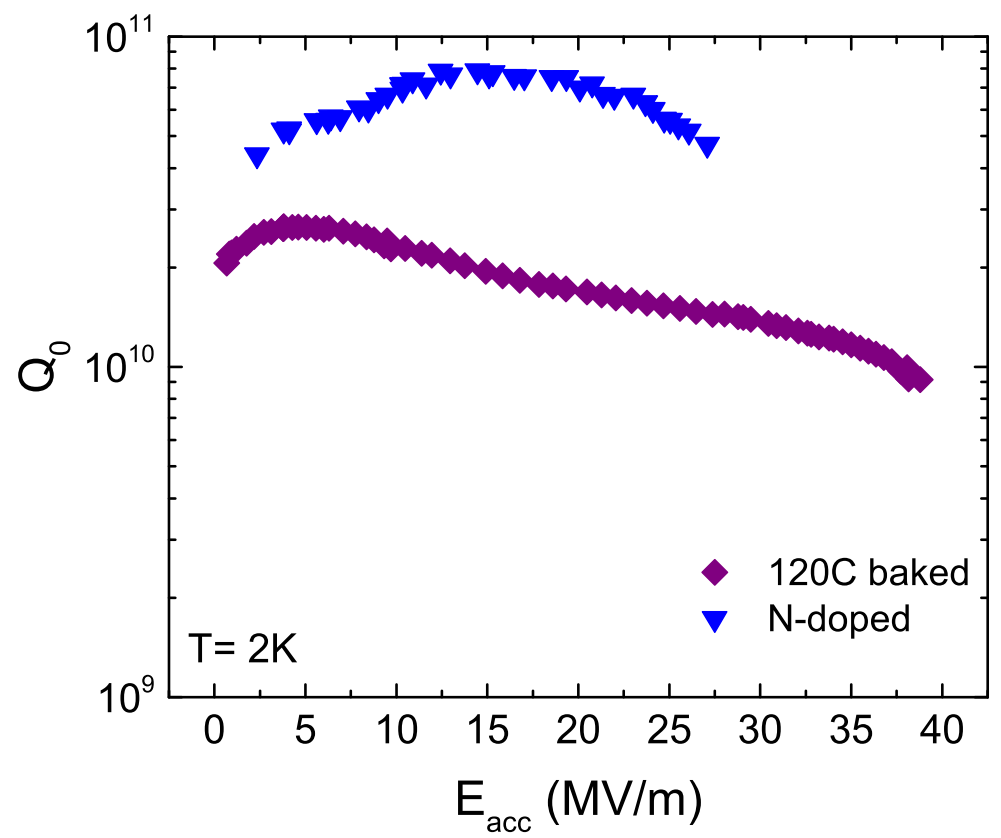

Figure 4.8. Q-factor versus accelerating field curve measured at $2 \mathrm{~K}$ for a N-doped and a $120{ }^{\circ} \mathrm{C}$ baked cavity.

\subsection{The nitrogen-doping treatment}

An even larger improvement in the SRF field was given by the discovery of the nitrogen doping, or N-doping, treatment. This surface treatment is capable to give ultra high value of Q-factors at medium field level. An example of Q-factor versus accelerating field curve measured at $2 \mathrm{~K}$ is shown in Figure 4.8, where it is compared with a curve of a standard $120^{\circ} \mathrm{C}$ baked cavity. The quality factor after the N-doping actually increases as a function of the accelerating field, until it reaches its maximum value around accelerating field of $15-20 \mathrm{MV} / \mathrm{m}$. Such peculiar behavior is called anti-Q-slope and is the typical signature of optimal N-doped cavities [19].

The nitrogen doping treatment is performed just after the $800{ }^{\circ} \mathrm{C}$ baking, when the cavity is still inside the UHV furnace. After this step nitrogen is injected with a partial pressure of about 25 mTorr and it is kept inside the furnace for some minutes at $800{ }^{\circ} \mathrm{C}$. During this step nitrogen reaches the cavity surface and reacts 


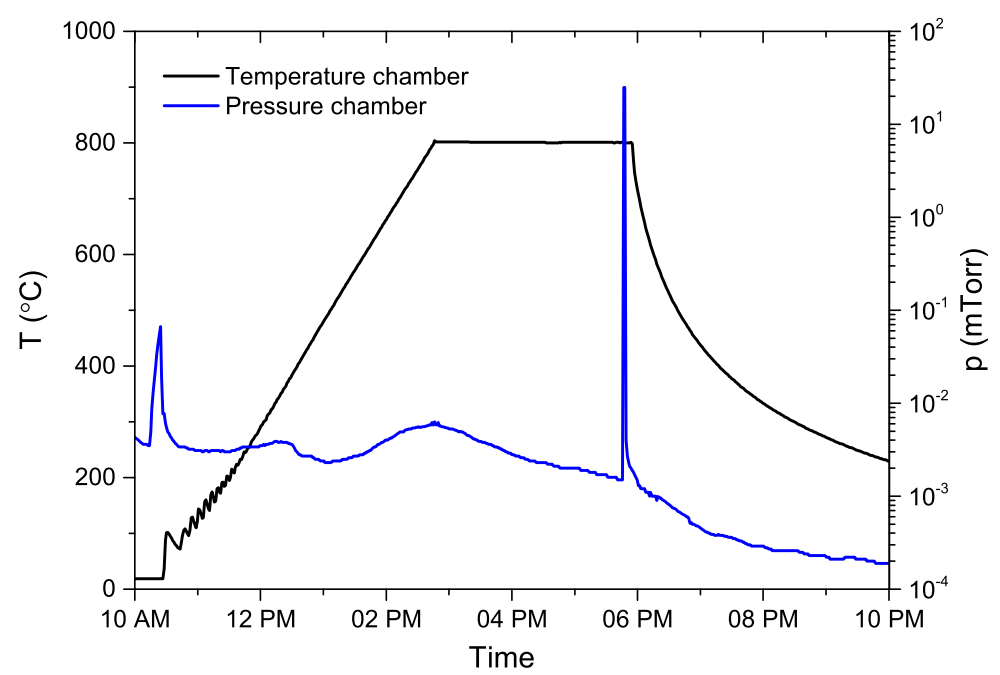

Figure 4.9. Temperature (black curve) and pressure (blue curve) of the chamber during the $2 / 6$ nitrogen doping treatment.

with niobium. This reaction causes the dissociation of nitrogen molecules in nitrogen atoms, that can be adsorbed from the niobium surface. Once nitrogen is absorbed diffuses deeper inside the niobium lattice.

The parameter of the process are: temperature, nitrogen partial pressure and time of nitrogen exposure. These parameters may be changed to modify the concentration and the depth profile of the nitrogen in the niobium cavity.

A second step may be added in order to extend the diffusion process. During this second step the nitrogen flow is shut off and the cavity stays at the same temperature, say $800{ }^{\circ} \mathrm{C}$, for some minutes in an UHV environment.

The temperature and pressure of the chamber recorded during a nitrogen doping treatment are shown in Figure 4.9. The N-doping treatment shown in the figure is called 2/6 N-doping, indicating that the nitrogen is kept inside the furnace for 2 minutes (first step), and then the nitrogen is shut off and the cavity is left for other 6 minutes at the same temperature but without nitrogen (second step).

In Figure 4.10, some Secondary Ion Mass Spectrometry (SIMS) spectra are 


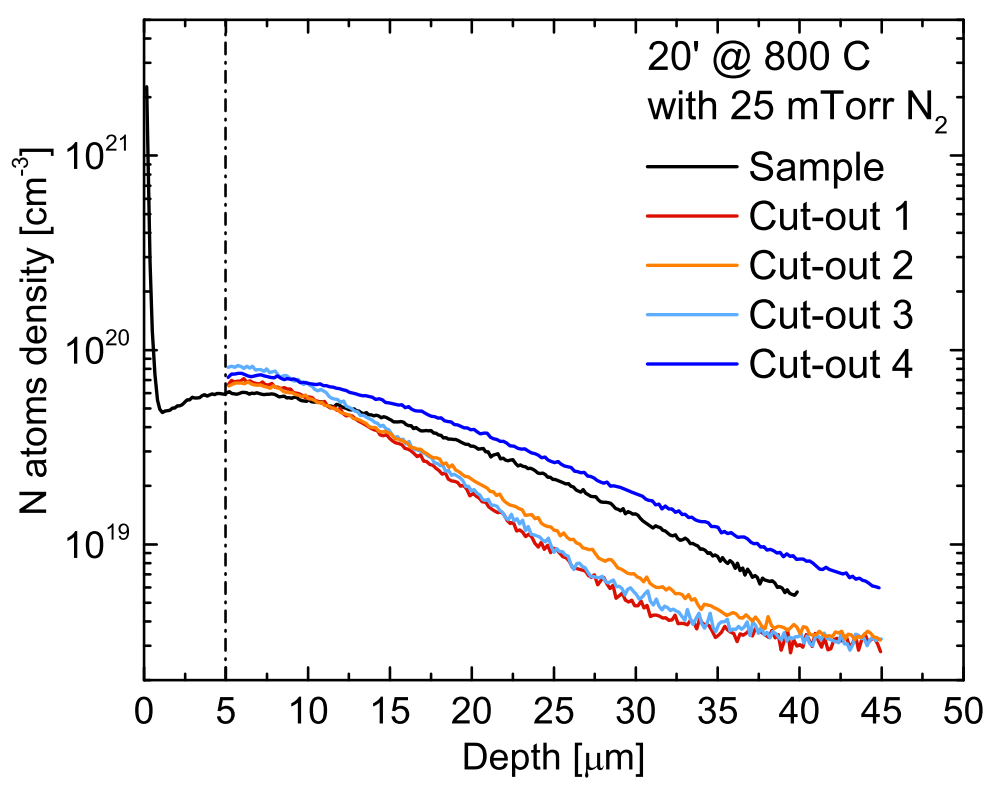

Figure 4.10. SIMS spectra of a N-doped sample and some cut-outs from the cavity ACC002. Both sample and cut-outs are measured after a 20 minutes N-doping treatment. The cavity cut-outs were also electro-polished removing $5 \mu \mathrm{m}$ from the surface.

shown. These were acquired for a sample and some cavity cut-outs (from the cavity ACC002). The sample was N-doped in the same way of the cavity ACC002, with a 20 minutes N-doping treatment. After the doping treatment, the cavity ACC002 has been electro-polished, removing the first $5 \mu \mathrm{m}$ from the surface. From the graph it is possible to notice that the sample show very high concentration of nitrogen within the first $1-2 \mu m$ of depth from the surface. This high concentration of nitrogen is attributed to non-stechiometric niobium nitrides phases that forms during the nitrogen treatment [53]. As it can be seen, the $5 \mu \mathrm{m}$ EP removal is effective in removing these niobium nitrides, that are bad superconductor and, if present, cause the increasing of the residual resistance [19]. Because of that, the EP removal of 5 $\mu \mathrm{m}$ from the surface is always done after the N-doping treatment.

In Reference [19], the surface resistance of N-doped cavities was decomposed in the two component BCS and residual resistance, in order to understand what is the origin of the anti-Q-slope. It was found that, surprisingly, the BCS surface resistance 
of N-doped cavities decreases as a function of the accelerating field, while for standard EP and $120{ }^{\circ} \mathrm{C}$ baked cavities $R_{\mathrm{BCS}}$ increases as a function of the accelerating field. This aspect is further studied in Chapter 7 of this thesis.

\subsection{Surface treatments of the studied cavities}

The 1.3 GHz single cell cavities studied during this thesis work were processed with different surface treatments, a summary is shown in Table 4.1. During all the treatments with nitrogen, or other gases, the partial pressure was wept at about 25 mTorr.

In case of N-doped cavities, in the surface treatment column is indicated the duration, in minutes, and temperature of the treatment. If also the second step of the N-doping treatment is performed, the surface treatment column indicates the duration of the first step / the duration of the second step, in minutes. When more treatments were done subsequently in the same cavity, a progressive number is added in the name, as: cavity name_treatment number.

The cavity AES018 was treated with helium but the final performance remained the same of a typical EP cavity, proving that helium does not diffuse in the niobium lattice for that range of pressure and temperature. Another peculiar treatment was done on cavity AES021 that was treated with air instead of pure nitrogen.

In Figure 4.11, the Q-factor versus accelerating field curves at $2 \mathrm{~K}$, of some of the studied cavities, are shown. Since at $2 \mathrm{~K}$ for most of the cavities the administrative limit of $17 \mathrm{MV} / \mathrm{m}$ was imposed in order to not quench the cavity, all the curves are shown till about the same level of accelerating field.

In Chapter 7, a full analysis of the RF surface resistance versus the mean free path will be shown for all these cavities. Important is to point out that from Figure 
Table 4.1. Summary of the surface treatments of the cavities studied in this thesis work.

\begin{tabular}{cc}
\hline \hline Cavity Name & Surface Treatment \\
\hline AES018 & 30 min He $\left(\right.$ at $\left.800{ }^{\circ} \mathrm{C}\right)$ \\
ACC005 & $120{ }^{\circ} \mathrm{C}$ bake \\
AES014 & $120 \mu \mathrm{m} \mathrm{EP}$ \\
AES014_2 & Additional $120{ }^{\circ} \mathrm{C}$ bake \\
AES005 & 1 hour $N_{2}\left(\right.$ at $\left.1000{ }^{\circ} \mathrm{C}\right)+60 \mu \mathrm{m} \mathrm{EP}$ \\
ACC002 & 20 min $N_{2}\left(\right.$ at $\left.800{ }^{\circ} \mathrm{C}\right)+5 \mu \mathrm{m} \mathrm{EP}$ \\
CBMM & $2 / 6$ min $N_{2}\left(\right.$ at $\left.800{ }^{\circ} \mathrm{C}\right)+5 \mu \mathrm{m} \mathrm{EP}$ \\
PAV009 & 20 min $N_{2}\left(\right.$ at $\left.900{ }^{\circ} \mathrm{C}\right)+5 \mu \mathrm{m} \mathrm{EP}$ \\
AES011 & $2 / 6$ min $N_{2}\left(\right.$ at $\left.800{ }^{\circ} \mathrm{C}\right)+5 \mu \mathrm{m} \mathrm{EP}$ \\
AES009 & $2 / 6$ min $N_{2}\left(\right.$ at $\left.800{ }^{\circ} \mathrm{C}\right)+5 \mu \mathrm{m} \mathrm{EP}$ \\
AES017 & $2 / 6$ min $N_{2}\left(\right.$ at $\left.800{ }^{\circ} \mathrm{C}\right)+5 \mu \mathrm{m} \mathrm{EP}$ \\
AES017_2 & Additional $2 / 6$ min $N_{2}\left(\right.$ at $\left.800{ }^{\circ} \mathrm{C}\right)+5 \mu \mathrm{m} \mathrm{EP}$ \\
AES019 & 10 min $N_{2}\left(\right.$ at $\left.800{ }^{\circ} \mathrm{C}\right)+5 \mu \mathrm{m} \mathrm{EP}$ \\
AES019_2 & Additional $50 \mu \mathrm{m} \mathrm{BCP}$ \\
AES019_3 & Additional 30 min $N_{2}\left(\right.$ at $\left.800{ }^{\circ} \mathrm{C}\right)+5 \mu \mathrm{m} \mathrm{EP}$ \\
AES021 & Air 30 min $\left(\right.$ at $\left.800{ }^{\circ} \mathrm{C}\right)+3 \mu \mathrm{m} \mathrm{EP}$ \\
AES021_2 & Additional $5 \mu \mathrm{m} \mathrm{EP}$ \\
\hline
\end{tabular}




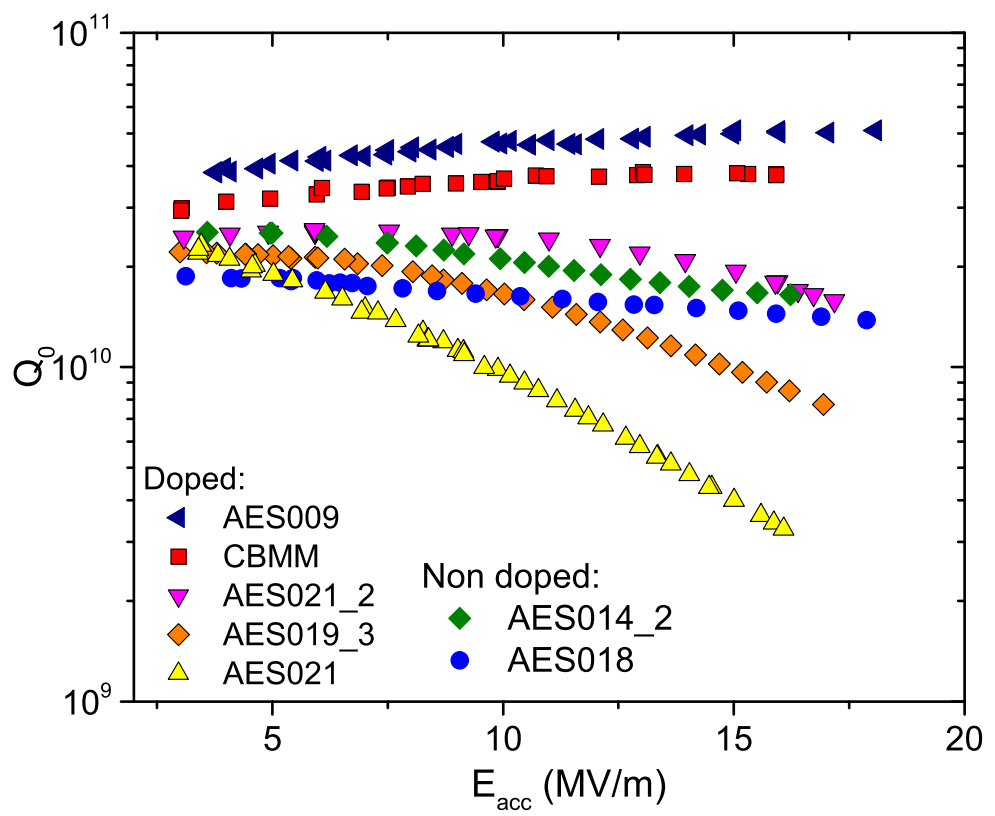

Figure 4.11. Q-factor versus accelerating field curves, measured at $2 \mathrm{~K}$, of some of the cavities studied.

4.11 it is possible to notice that some N-doping treatments are not optimal. For example, cavities AES021 and AES019_3 show an important Q-slope starting from low field level. Both these cavities are heavily doped as can be seen from Table 4.1. These kind of heavily doped treatments are called over-doped, and a possible explanation of their bad performance may be the non-optimal concentration of dopant in the lattice, or the presence of non-stechiometric nitrides, or other bad superconducting phases at the surface, that may have not be fully removed with the EP treatment. Indeed, cavity AES021 showed better performance after an additional $5 \mu \mathrm{m}$ EP removal (AES021_2 in Figure 4.11), likely because spurious non-superconducting phases have been removed from the RF surface, as suggested previously.

Measuring the cavity Q-factor at both $2 \mathrm{~K}$ and $1.5 \mathrm{~K}$, it is possible to decompose the BCS surface resistance from the residual resistance contribution, indeed:

$$
R_{\mathrm{s}}(2 K)=R_{\mathrm{BCS}}(2 K)+R_{\mathrm{res}} \quad, \quad R_{\mathrm{s}}(1.5 K) \simeq R_{\mathrm{res}}
$$

therefore: $R_{\mathrm{BCS}}(2 K)=R_{\mathrm{s}}(2 K)-R_{\mathrm{s}}(1.5 K)$ and $R_{\text {res }} \simeq R_{\mathrm{s}}(1.5 K)$. The result of this 


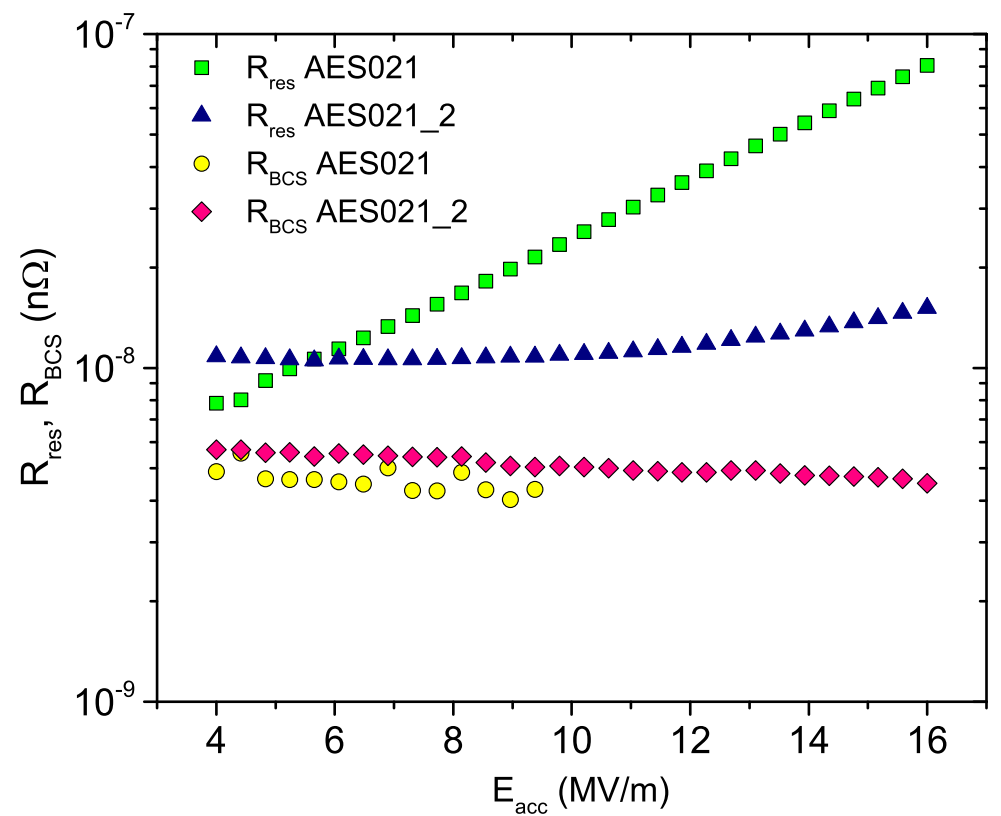

Figure 4.12. Surface resistance decomposition in BCS and residual resistance contributions for the cavity AES021.

decomposition for cavity AES021 is shown in Figure 4.12. AES021_2 indicates the result from the same cavity after $5 \mu \mathrm{m}$ of EP removal. It is possible to notice that the Q-slope of Figure 4.11 is due to the increasing of the residual resistance contribution with the field. After the $5 \mu \mathrm{m}$ of EP removal the residual resistance contribution becomes almost constant as a function of the accelerating field. The BCS surface resistance contribution is instead really low and it slightly decreases as a function of the accelerating field, as expected from N-doped cavities. 


\section{CHAPTER 5}

\section{MAGNETIC FLUX EXPULSION IN HORIZONTALLY COOLED CAVITIES}

\subsection{Introduction}

Trapped magnetic flux in superconducting resonators contributes to radiofrequency $(\mathrm{RF})$ surface resistance $\left(R_{\mathrm{s}}\right)$, in the form of the temperature-independent resistance called residual resistance $R_{0}[25]$.

The residual resistance due to trapped flux plays an important role in superconducting radio-frequency $(\mathrm{SRF})$ cavity performance, potentially degrading the efficiency of the cavity. Recent studies $[54,5]$ have shown that performing fast cooldowns, with large thermal gradients along the cavity, is vital to obtain efficient magnetic flux expulsion, and that slow and homogeneous cooling through transition leads to full flux trapping.

The speed of the cooldown determines the thermal gradient along the cavity length during the superconducting transition, which seems to be the key parameter that establishes the final amount of trapped flux.

In Reference [5] the residual resistance of the same cavity was acquired under different cooldown condition, but comparable external magnetic field. The graph of residual resistance as function of the thermal gradient $T_{1}-T_{2}$ (Figure 5.1) highlights how the residual resistance strongly depends on the cooldown detail. Here $T_{1}$ is the temperature at the top cavity position and $T_{2}$ is the temperature at the equator, and their difference is measured when $T_{1}=9.2 \mathrm{~K}$, i.e. when the equator becomes superconducting. During the experiment described in Reference [5], the cavity is vertically placed with respect to the cryostat axis and the helium flow. The vertical

cooldown is the one generally used to test SRF cavities performance, however, cavities in accelerators are horizontally placed with respect to the helium flow. 


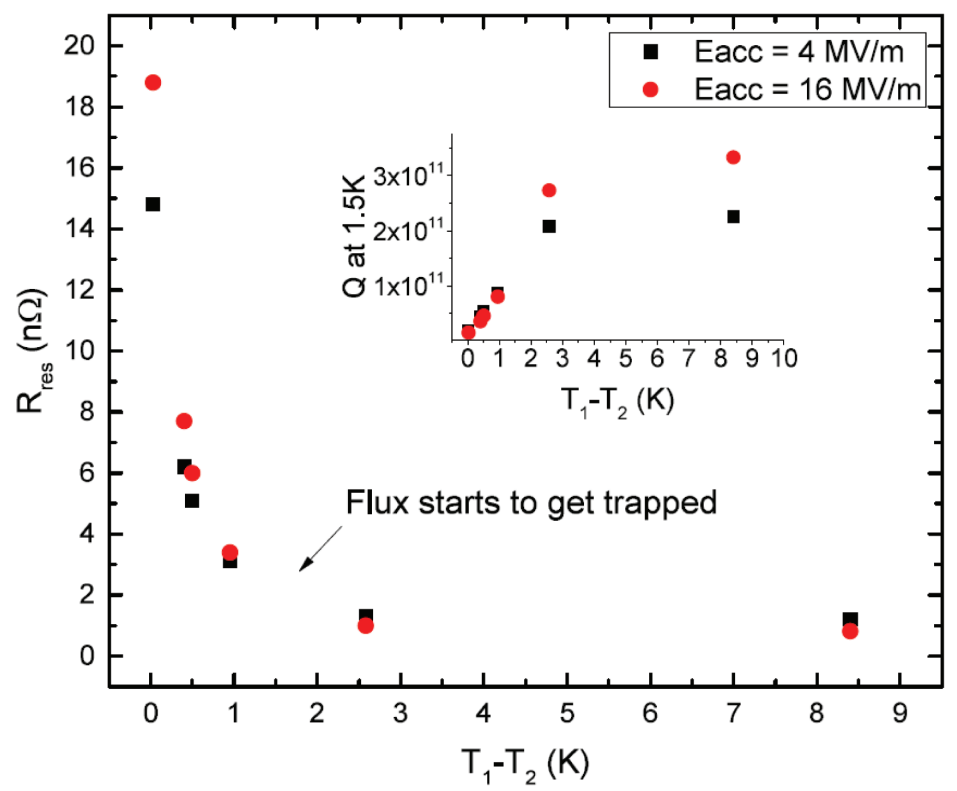

Figure 5.1. Residual resistance at as a function of the thermal gradient $T_{1}-T_{2}$ between equator $T_{2}$ and top iris $T_{1}$ when the equator become SC. The inset shows the corresponding $Q_{0}$ values [5].

For this reason in this chapter the cooldown is studied placing the cavity horizontally with respect to the cryostat axis. The geometry of the cavity cooling in real accelerator is in this way resembled.

During a fast cooldown the liquid helium is injected from the bottom of the cryostat, which is then filled fast until all the thermal sensor attached to the cavity indicates temperature well below $T_{\mathrm{c}}$.

When the fast cooldown is performed with the cavity oriented horizontally with respect to the cryostat axis, the boundary between the superconducting (SC) and the normal conducting (NC) phases will move from the very bottom to the very top point of the cell equator, rather than from beam tube to beam tube as in vertical orientation studies. A scheme of such configurations is shown in Figure 5.2.

Conversely, during a slow cooldown, the gaseous helium is injected from both bottom and top of the cryostat in order to make the cavity temperature as more 


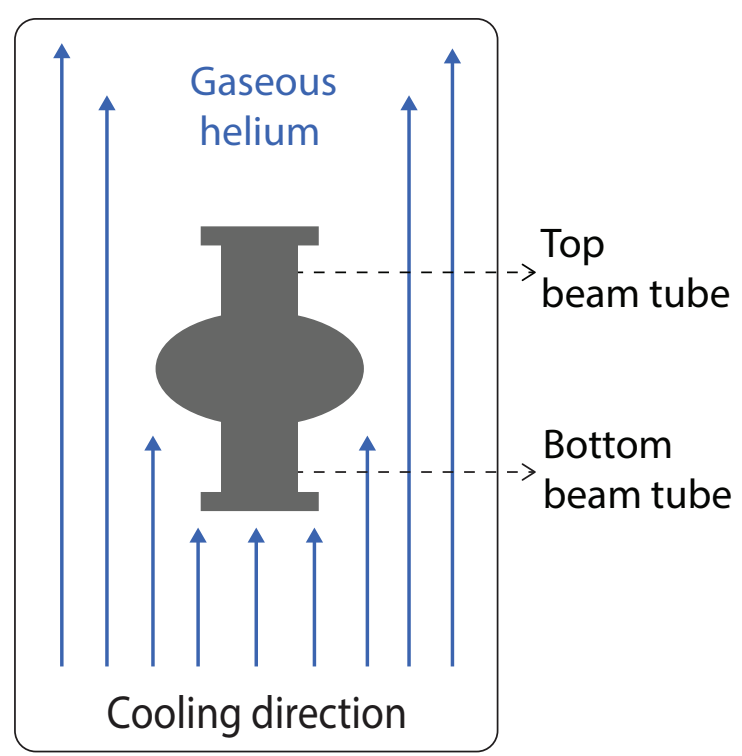

(a)

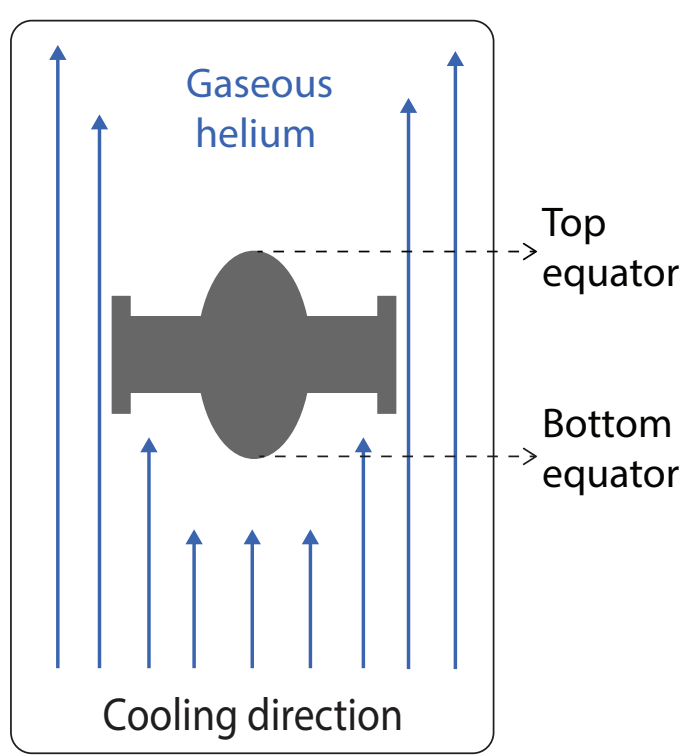

(b)

Figure 5.2. Sketch of the two cooldowns orientation: a) vertical and b) horizontal.

uniform as possible during the SC transition.

Several continuous wave (CW) accelerators currently being built worldwide (for example x-ray FELs such as LCLS-II at SLAC $[55,56]$ ) require very high Qfactors, highly efficient SRF cavities, to reduce cryogenic costs. Therefore, the understanding of the cooldown dynamics in a configuration that resembles the cavity in an accelerator, in the presence of magnetic field levels comparable to those present in shielded cavities placed in a cryomodule, is crucial in order to investigate how to minimize the surface losses due to trapped flux.

\subsection{Experimental set-up}

A single cell $1.3 \mathrm{GHz}$ TESLA type nitrogen doped bulk niobium cavity was used for this study, which is the same cavity used in Reference [5]. It is worth mentioning that this cavity has achieved world record quality factors $Q_{0}>1 \cdot 10^{11} \mathrm{up}$ to the highest fields $30 \mathrm{MV} / \mathrm{m}$ at $1.5 \mathrm{~K}$ and with a $Q_{0}>5 \cdot 10^{10}$ up to $30 \mathrm{MV} / \mathrm{m}$ at 


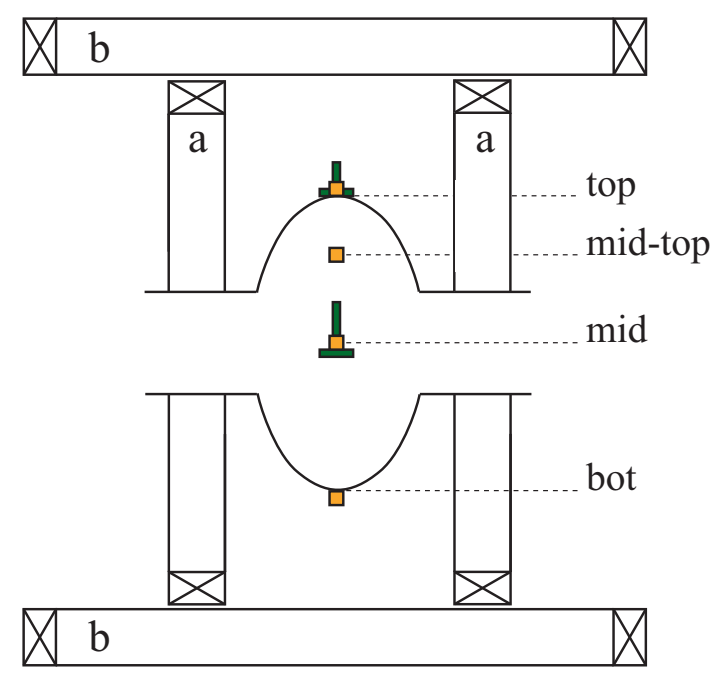

Figure 5.3. Horizontal cooldown cavity set-up. The orange squares and the green rectangle represents respectively the thermometers and the fluxgates, while a and b stand for the two Helmholtz coils.

$2 \mathrm{~K}$.

The set-up and the instrumentation of this experiment are shown in Figure 5.3. Two pairs of Helmholtz coils were placed orthogonally one to each other (Figure 5.3), one parallel to the cavity axis (a) and the other perpendicular to it (b). In addition, four Cernox thermometers were placed at the cavity equator (orange squares in Figure $5.3)$ in the following position: bottom of the cell, middle, top, and one half way in between the top and the middle ones.

The external magnetic field applied to the cavity was measured by means of four single-axis Bartington Mag-01H cryogenic fluxgate magnetometers (green rectangles in Figure 5.3). Two of them were placed perpendicularly to the cavity axis, one at the very top of the cell and one at the middle, while the other two were placed at the same positions but axially to the cavity axis.

Several fast cooldowns were performed under different magnetic field orientations (orthogonal or axial) with the same magnitude, about $10 \mathrm{mG}$. In order to obtain different thermal gradients across the cavity, different starting temperatures 
were chosen for these fast cooldowns.

\subsection{Horizontal cooldown data analysis}

5.3.1 RF measurements analysis. RF measurements were performed at the Fermilab SRF cavity vertical test facility. The unloaded Q-factor $\left(Q_{0}\right)$ versus accelerating field $\left(E_{\text {acc }}\right)$ curves were acquired at $2 \mathrm{~K}$ and at the lowest temperature achievable with the pumping system, which is slightly lower than the calibration range of the thermometers $(T<1.4 \mathrm{~K})$. At such low temperatures, the surface resistance is dominated by the temperature-independent part (residual resistance, $R_{0}$ ). Therefore, at this temperature the differences in terms of losses due to trap flux, which affects only $R_{0}$, are well visible.

The $Q_{0}$ versus accelerating field curves acquired at $T<1.4 \mathrm{~K}$ are shown in Figure 5.4, while the cooldown conditions of the data series are summarized in Table 5.1. As studied in previous work [44], the uncertainty of the measurement of $Q_{0}$ does not exceed $10 \%$.

The cooldowns of the data series named nAx (axial) were performed applying $10 \mathrm{mG}$ of external axial magnetic field. For curves $1 \mathrm{Ax}$ and $2 \mathrm{Ax}$ an administrative limit of $16 \mathrm{MV} / \mathrm{m}$ was set for the accelerating field to avoid quenching the cavity.

Table 5.1. Cooldowns summary and associated measured residual resistance.

\begin{tabular}{cccc}
\hline \hline Name & Field Orientation & Starting $\mathrm{T}(\mathrm{K})$ & $R_{0}(\mathrm{n} \Omega)$ \\
\hline $1 \mathrm{Ax}$ & Axial & 300 & 2.1 \\
$2 \mathrm{Ax}$ & Axial & 50 & 5.3 \\
$3 \mathrm{Ax}$ & Axial & 300 & 2.9 \\
\hline 1 Ort & Orthogonal & 300 & 6.3 \\
2 Ort & Orthogonal & 260 & 6.1 \\
3 Ort & Orthogonal & 170 & 7.7 \\
4 Ort & Orthogonal & 25 & 13.9 \\
\hline
\end{tabular}




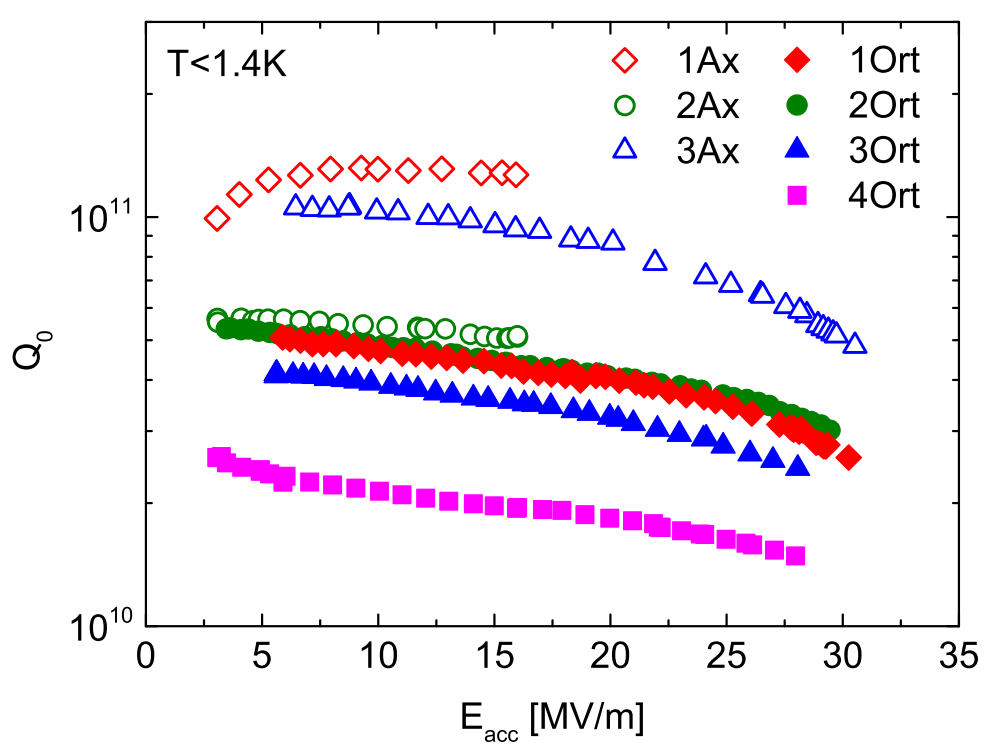

Figure 5.4. $Q_{0}$ versus accelerating field measured at $T<1.4 \mathrm{~K}$.

The highest quality factor was reached with 1Ax: the Q-factor increases slightly at low field and it reaches $1.3 \cdot 10^{11}$ at $16 \mathrm{MV} / \mathrm{m}$. The $3 \mathrm{Ax}$ data set reveals a reduced performance. $Q_{0}$ decreases considerably with the accelerating field, showing the typical slope due to trapped magnetic flux $[57,5]$. The worst performance for the axial series is found for the $2 \mathrm{Ax}$ data set, in which the Q-factor reaches only $5.1 \cdot 10^{10}$ at $16 \mathrm{MV} / \mathrm{m}$.

The cooldowns of the nOrt (orthogonal) series were performed applying $10 \mathrm{mG}$ orthogonally to the cavity axis. In general, all the curves of the orthogonal series show reduced performance compared to the axial series, and they all show field dependent Q-slopes characteristic of trapped flux. The best performance for the orthogonal series is shown by 2 Ort with $Q_{0}=4.3 \cdot 10^{10}$ at $16 \mathrm{MV} / \mathrm{m}$, while the lowest Q-factor values are given by the 4 Ort data series, and in this case the Q-factor is $1.9 \cdot 10^{10}$ at $16 \mathrm{MV} / \mathrm{m}$.

The residual resistance at $16 \mathrm{MV} / \mathrm{m}$ was calculated directly as $R_{\text {res }}=G / Q_{0}$ (with $G=270 \Omega$ ), since, as already mentioned, the surface resistance at $T<1.4 \mathrm{~K}$ 


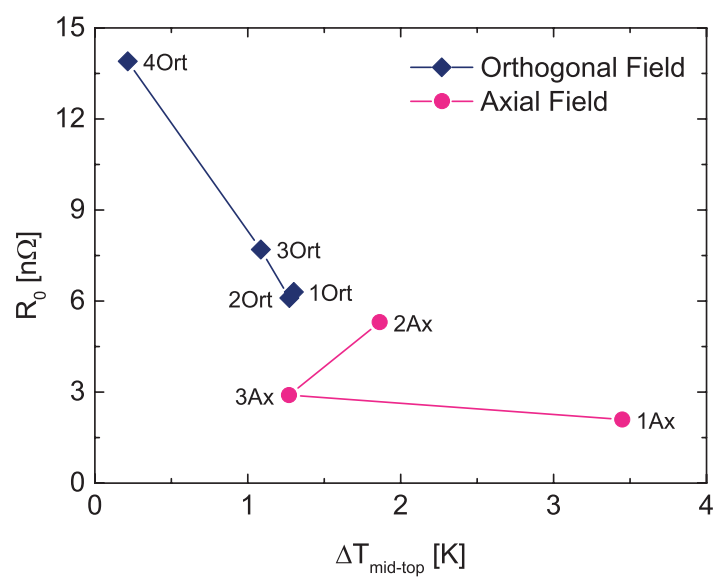

a)

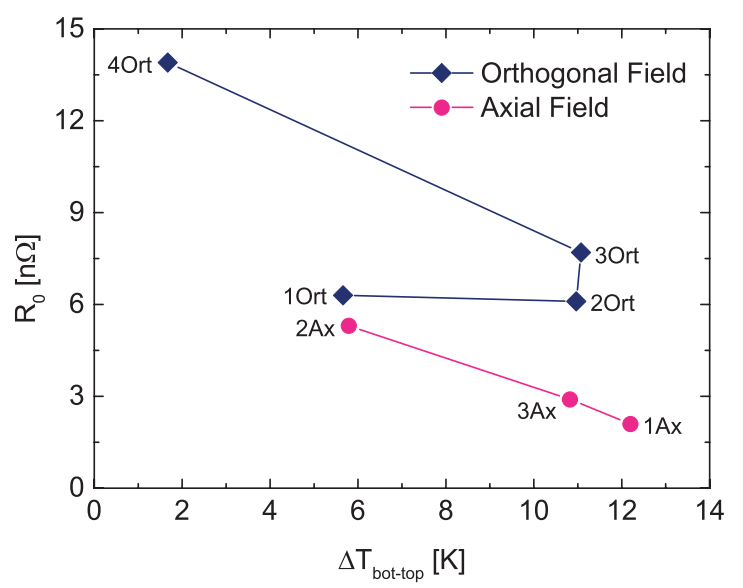

b)

Figure 5.5. Residual resistance versus mid-top (a) and bot-top (b) thermal gradients [6].

is affected only by the residual part. These values of residual resistance, reported in Table 5.1, allows to compare the global cavity losses of each series.

5.3.2 Cooldown dynamics analysis. Examining the data series axial and orthogonal separately, different cooldowns lead to different residual resistances, as reported previously for the usual vertical configuration [5].

Two parameters seem useful to describe the dynamics of the cavity cooldown: the temperature differences $\Delta T_{\text {bot-top }}$ and $\Delta T_{\text {mid-top }}$. The first one corresponds to the temperature difference between the top and the bottom of the cell, when the bottom reaches $T_{c}$. The second one is the temperature difference between the top and the mid positions of the cell when the middle position passes through the SC transition.

The residual resistance as a function of the thermal gradients $\Delta T_{\text {mid-top }}$ and $\Delta T_{\text {bot-top }}$ is displayed in Figure 5.5. Looking at Figure 5.5 (a), the residual resistance, for the orthogonal series, seems to follow a trend with the thermal gradient $\Delta T_{\text {mid-top }}$. No particular trend appears for the axial series. On the other hand, the linear trend of the residual resistance as a function of the thermal gradient $\Delta T_{\text {bot-top }}$ appears only for the axial series (Figure $5.5(\mathrm{~b})$ ). 


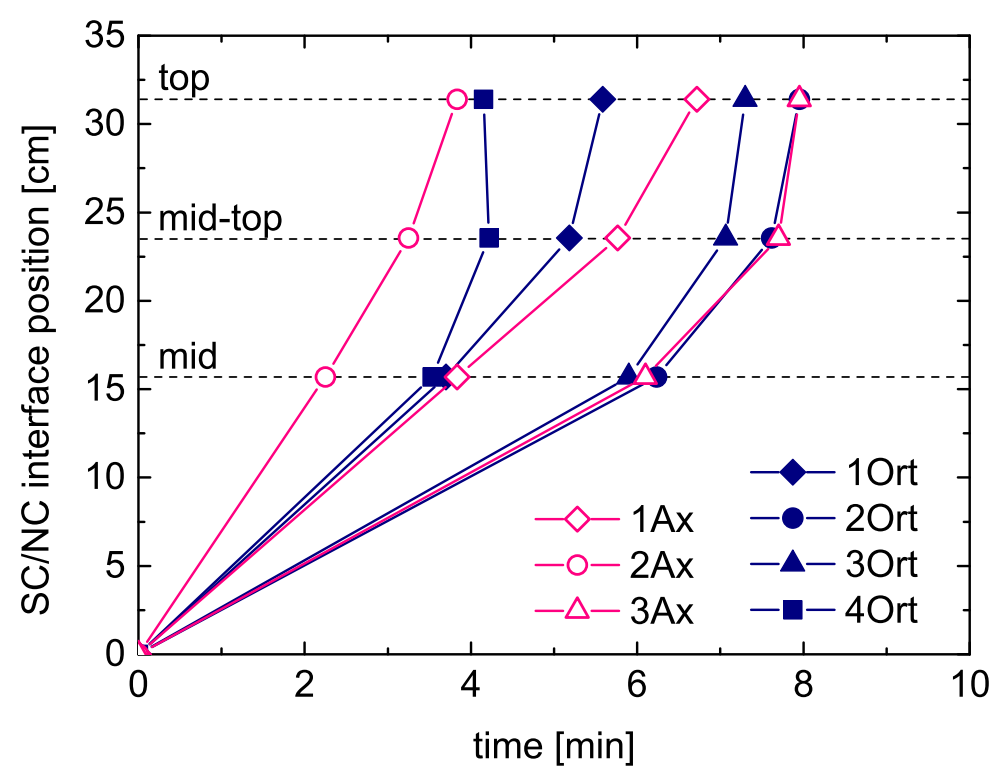

Figure 5.6. Evolution of the SC-NC interface during the cooldown [6].

Comparing 2Ort and 3Ort, they show the same $\Delta T_{\text {bot-top }}$ thermal gradients, but lower $R_{0}$ value is measured for 3Ort, which instead has a higher $\Delta T_{\text {mid-top }}$.

The data suggests that both mid-top and bottom-top thermal gradients may play an important role to determine the residual losses. As one could intuitively expect: cooling details may vary as the SC-NC boundary progresses along the cavity profile, but what matters for efficient flux expulsion are thermal gradients at the SCNC phase front present during the whole period of time when the transition front progresses through the cavity.

In order to better understand the global behavior of the cavity during the cooldown, the SC-NC interface evolution has been investigated. Setting to zero the time at which the cavity bottom position passes through transition, the position of the SC-NC interface can be plotted against the time it takes moving from one thermometer position to another, as shown in Figure 5.6.

It is important to underline that the slope of the segment connecting two points 
corresponds to the average speed $(\mathrm{cm} / \mathrm{min})$ of the SC-NC interface along the cell, that should not be confused with the cooling speed $(\mathrm{K} / \mathrm{min})$ which, on the contrary, does not seem to be a key parameter for cavity losses.

From Figure 5.6 it is possible to conclude that thermal gradients per unit length are not constant throughout the movement of the SC-NC interface, but they decrease as the boundary moves towards the top. This is perhaps an effect of the cavity starting out warm but then rapidly cooling by conduction. This could potentially cause more flux to get trapped at the top, which in the horizontal cavity case corresponds to the equator, causing a greater performance degradation compared to the vertical cavity orientation.

As an extreme case, it can be noticed that for the series 4Ort the top of the cavity passes through transition before the mid-top position. The last point which becomes SC is not the very top as in all the other cases, therefore the trapped flux is not concentrated at the equator but rather is redistributed in the nearby zone. The fact that the mid-top position becomes superconducting after the very top of the cavity suggests that the SC transition dynamic does not follow a sharp SC-NC interface movement across the cavity, but is rather described by random nucleation of SC island. In this nucleation scenario, the incomplete Meissner effect is enhanced by the presence of normal conducting islands surrounded by SC material, leading to a reduced efficiency of flux expulsion and large residual losses, as hypothesized in previous work [54] and demonstrated in Chapet 6.

Looking carefully at the series $3 \mathrm{Ax}$ and 2Ort, they show the same $\Delta T_{\text {bot-top }}$ and $\Delta T_{\text {mid-top }}$ thermal gradients (Figure 5.5), and also the same SC-NC transition dynamics (Figure 5.6). This implies that the two cooldowns can be considered comparable, and the difference by a factor of 2 in the residual resistance between them can be more likely attributed to some effects introduced by the different orientation 


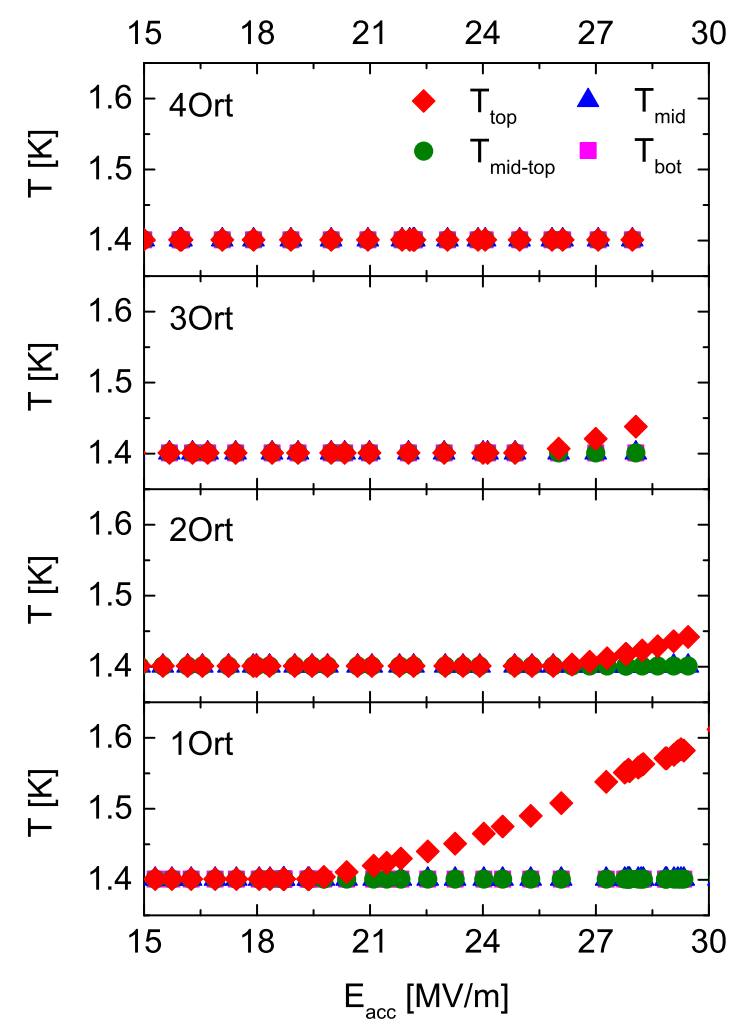

Figure 5.7. Temperature variation versus the accelerating field [6].

of the magnetic field.

It is important to point out that the magnetic field just before the transition was slightly higher in case of the 2 Ort (about $12 \mathrm{mG}$ ) than in the case of $3 \mathrm{Ax}$ (about $9 \mathrm{mG}$ ), yet this difference does not seem sufficient to account for a factor of two difference in residual resistance per same cooling regime. In general, looking at Figure 5.4 and at the residual resistances listed in Table 5.1, seems that the magnetic field applied orthogonally to the cavity axis may have a larger effect in deteriorating the cavity performance and increasing the residual losses than the axial magnetic field.

5.3.3 Flux hole scenario. In Figure 5.7 is shown the temperature acquired at the bottom, mid, mid-top and top positions of the cavity equator, as a function of the accelerating field. The figure shows that the orthogonal magnetic field can 


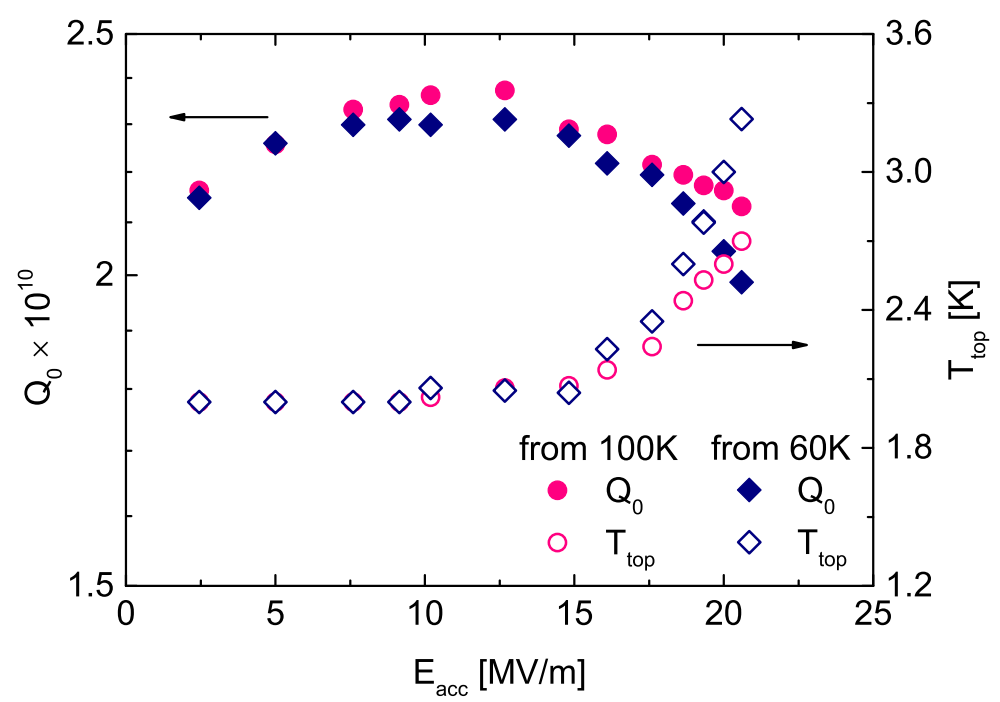

Figure 5.8. $Q_{0}$ and $T_{\text {top }}$ versus accelerating field for HTS measurements done with cooldowns started at $100 \mathrm{~K}$ and $60 \mathrm{~K}$ [6].

lead to local heating on top of the cavity equator, meaning that the magnetic field concentrates in this area after the SC transition. Indeed, during the acquisition of the series 1Ort, 2Ort and 3Ort the thermometers at the top position warmed up at high field, as shown in Figure 5.7. This temperature rising is prominent in the 1Ort series, where the temperature starts to exceed $1.4 \mathrm{~K}$ at about $20 \mathrm{MV} / \mathrm{m}$, and it reaches 1.6 $\mathrm{K}$ at about $30 \mathrm{MV} / \mathrm{m}$. The warming up is lower for the 2 Ort and 3Ort series where it starts from about $27 \mathrm{MV} / \mathrm{m}$ reaching just $1.45 \mathrm{~K}$. The absence of heating of 4 Ort may be due to the different cooldown dynamics, as discussed previously, which does not involve the concentration of magnetic flux at the very top of the cavity, but rather flux being homogeneously trapped because of lack of cooling thermal gradients.

In the other cases instead, the heating on the very top position of the cavity is a newly described phenomenon for SRF cavities, and it can be considered a proof of the local dissipation due to concentrated trapped flux on top of the cavity equator that appears when cavities are cooled in horizontal configuration, and in presence of orthogonal magnetic field. 
Interestingly, the same effect was repeatedly observed during test of the 9-cell nitrogen-doped niobium cavity TB9AES021 dressed with the LCLS-II vessel at the FNAL horizontal test facility (HTS). This cavity was instrumented with flux gates and thermometers inside the helium vessel, and as shown in Figure 5.8, the thermometer located on top of cell 1 of the cavity (input coupler side) showed significant heating starting at medium field (about $10 \mathrm{MV} / \mathrm{m}$ ) and the temperature reached values larger than $3 \mathrm{~K}$ at above $20 \mathrm{MV} / \mathrm{m}$. This heating strongly affected cavity performance, causing $Q_{0}$ degradation.

As can be seen in Figure 5.8, increasing the cooldown starting temperature pushes the onset of the heating and correspondingly improves cavity performance. Note that the increasing of the starting temperature usually traduces in larger thermal gradients along the cavity. This nine cell data, together with the previously presented single cell data suggests a scenario where an orthogonal magnetic field component might be present close to cell 1 during the SC-NC transition, causing a "flux hole" hot spot to appear on top of cell 1, and suggesting that this is an important performance limiting mechanism for superconducting cavities placed in accelerators.

5.3.4 Flux expulsion analysis. In order to further analyze this phenomenon, the magnetic field data acquired during the single-cell cavity horizontal cooldown are analyzed.

The magnetic field data acquired during the cooldowns are shown in Figure 5.9 (axial magnetic field series) and 5.10 (orthogonal magnetic field series). In order to properly interpret these data it is necessary to take into account that, because of the Meissner effect, after an efficient flux expulsion the magnetic field redistributes outside the superconductor depending on its geometry.

In case of an elliptical single-cell cavity with field parallel to its beam axis, the 

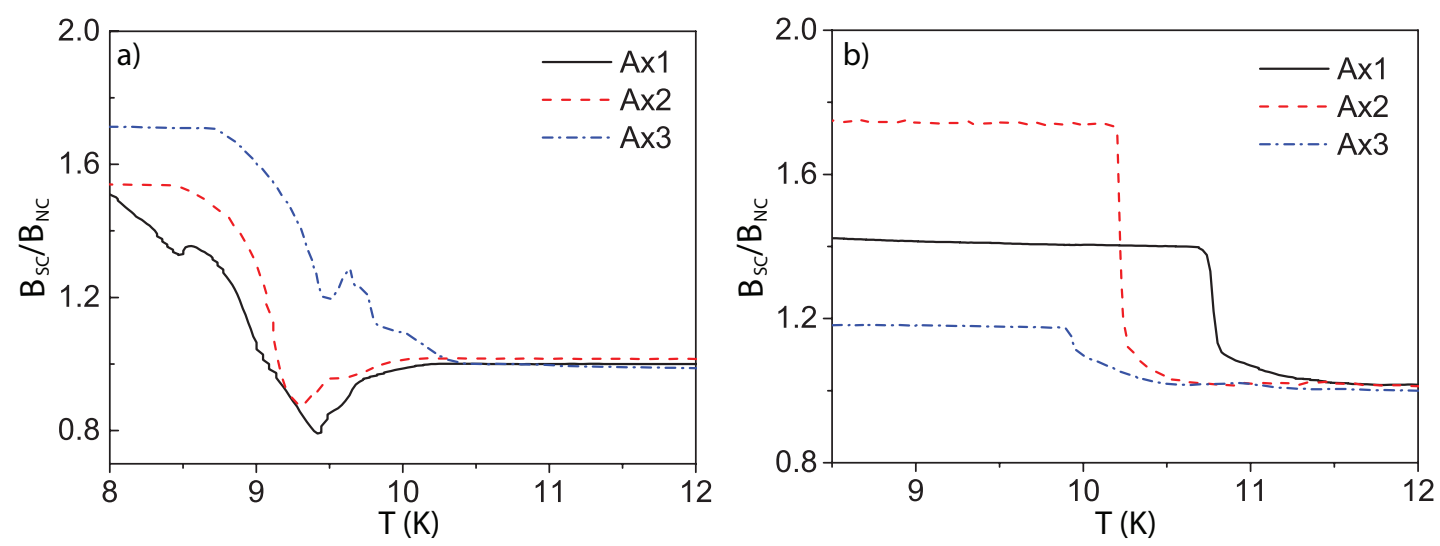

Figure 5.9. Ratio between the magnetic field measured after $\left(B_{\mathrm{SC}}\right)$ and before $\left(B_{\mathrm{NC}}\right)$ the SC transition for the axial magnetic field series. $B_{\mathrm{SC}} / B_{\mathrm{NC}}$ measured with the fluxgate at the cavity mid position (a) and with the fluxgate at the cavity top position (b).
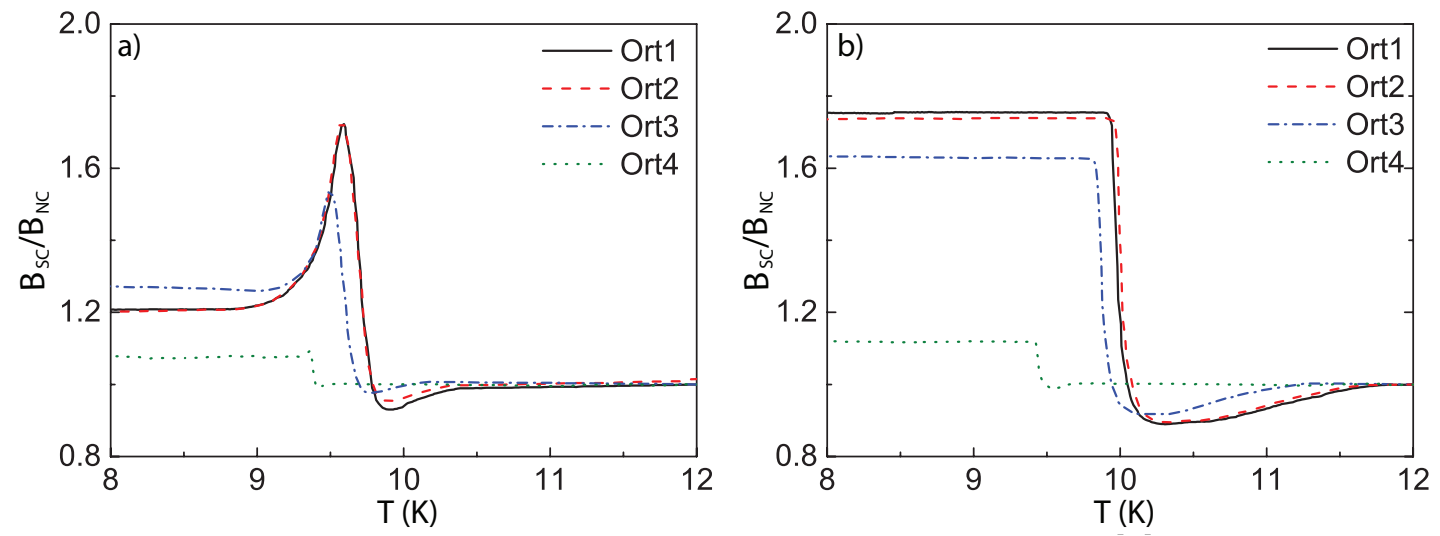

Figure 5.10. Flux expulsion ratio $B_{\mathrm{SC}} / B_{\mathrm{NC}}$, for the orthogonal magnetic field series, measured with the fluxgate at the cavity mid position (a) and with the fluxgate at the cavity top position (b) [6].

magnetic field lines thickened at the equator. In the ideal case, when the magnetic field is completely expelled from the cavity walls, the magnetic field at the cavity equator increases by approximately a factor of 1.74 when passing from the NC to the SC state (Figure 5.11). The cylindrical symmetry of the system implies that the magnitude of the field after the SC transition is the same all around the equator.

This means that when the cavity expels some magnetic field, a step should appear in the magnetic field data at the moment of the SC transition. On the other 


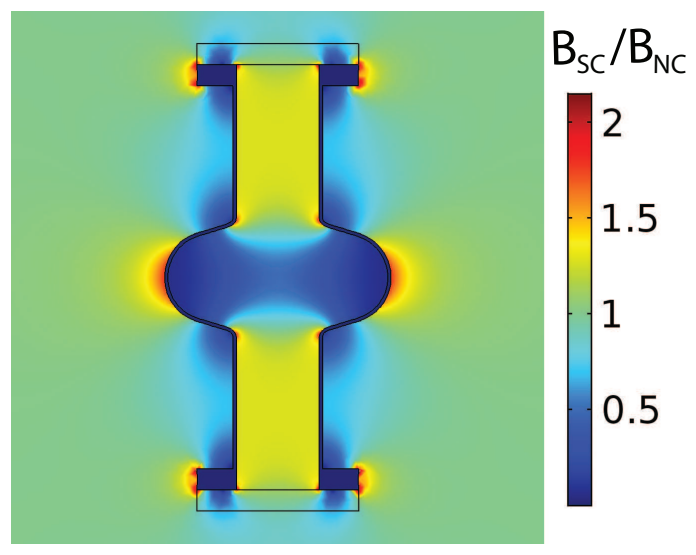

Figure 5.11. COMSOL simulation of the magnetic field redistribution outside the cavity due to the Meissner effect, considering axial magnetic field applied.

hand, when no step appears during the transition means that all the magnetic field get trapped in the cavity during the cooldown.

The magnetic field step occurs always at the $\mathrm{SC}$ transition temperature $T_{c}$, but from Figure 5.9 it seems to be different from one series to another, this is possibly due to the imperfect thermal equilibrium between cavity and thermometers, especially for fast cooldowns with large starting temperatures.

In Figure 5.9 the ratio between the axial magnetic field after $\left(B_{\mathrm{SC}}\right)$ and before $\left(B_{\mathrm{NC}}\right)$ the $\mathrm{SC}$ transition is shown as a function of temperature, for the mid (a) and top positions (b). When the mid position pass through the SC transition, good magnetic flux explosion is recorder for all the three cooldowns. At the top position instead only Ax2 fully expelled the magnetic flux and for Ax3 the expulsion was instead poor. This flux expulsion data are not fully in agreement with residual resistance values. This is another proof that during the horizontal cooldown the magnetic flux expulsion is driven by the local thermal gradient and therefore in order to obtain a clear picture of the amount of flux expelled it would be necessary to have more flux gates along the cavity equator.

In case of orthogonal field applied, the magnetic field redistributes differently 


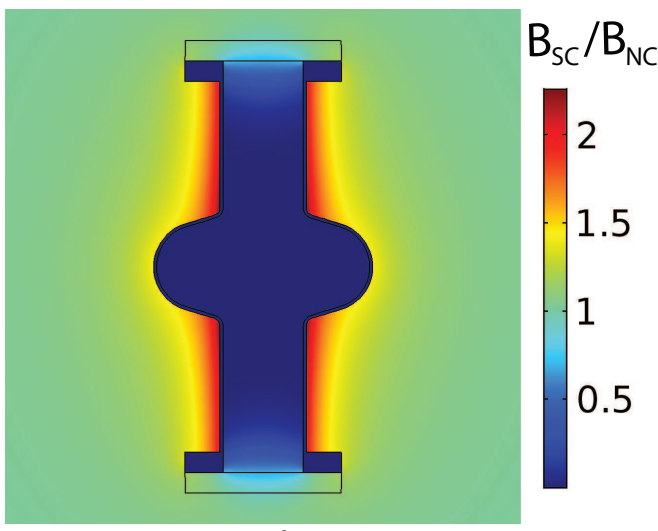

a)

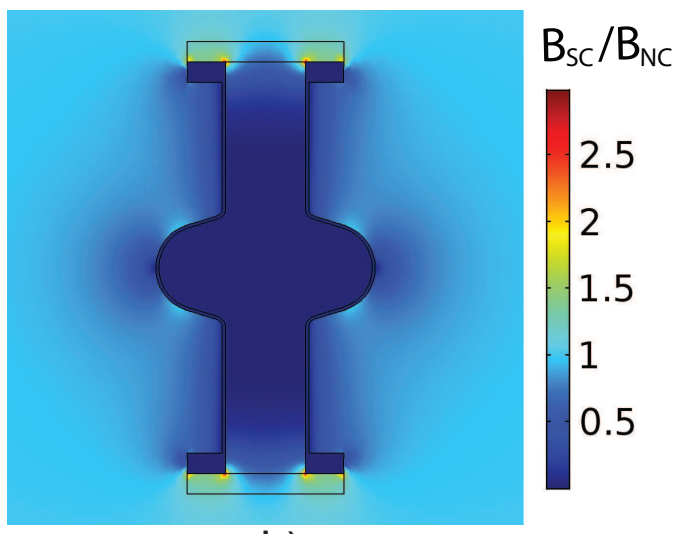

b)

Figure 5.12. a) COMSOL simulation of the magnetic field redistribution, along the yxplane, considering orthogonal magnetic field applied (along the z-axis). b) COMSOL simulation of the magnetic field redistribution, along the zx-plane, considering orthogonal magnetic field applied (along the z-axis).

than in the previous case. In addition, in this case the cylindrical symmetry is broken. Assuming that the cavity axis is along the $\mathrm{x}$-axis and the orthogonal magnetic field is applied along the z-direction, it is necessary to analyze the redistribution along both the xy and xz planes in order to understand the magnetic field value reads from the orthogonal mid and top fluxgates. When the magnetic flux is completely expelled from the cavity walls, the magnetic field at the cavity mid position increases by approximately a factor of 1.4 (Figure 5.12 (a)), while it tends to zero at the top position (Figure $5.12(\mathrm{~b})$ ).

In Figure 5.10 (a) the ratio $B_{\mathrm{SC}} / B_{\mathrm{NC}}$, acquired with the orthogonal fluxgate at the mid position of the cavity, is shown as a function of temperature. The series Ort1, Ort2, and Ort3 show good flux expulsion while 4Ort shows poor flux expulsion. In Figure 5.10 (b) the ratio $B_{\mathrm{SC}} / B_{\mathrm{NC}}$, acquired with the orthogonal fluxgate on top, as a function of temperature, gives instead information regarding the field trapped on this position. This data suggest that Ort1, Ort2, and Ort3 trapped a considerably larger amount of magnetic field on top of the cavity equator with respect to 4 Ort. 


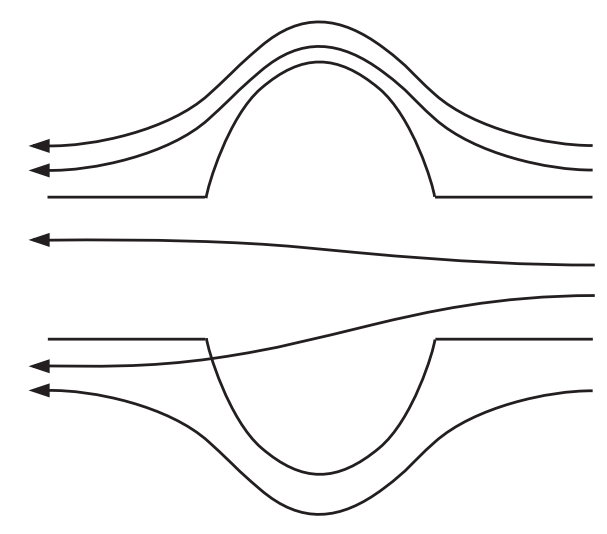

a)

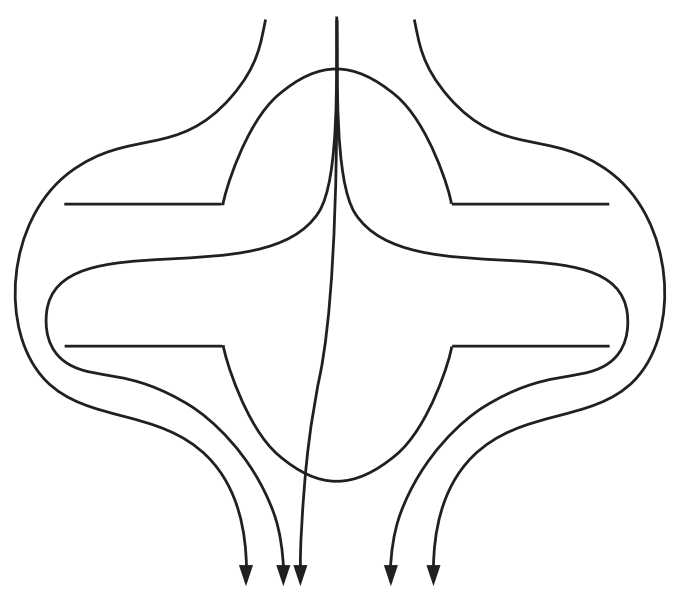

b)

Figure 5.13. Sketch of the field redistribution in the Meissner state with magnetic field applied a) axially and b) orthogonally to the cavity axis [6].

The magnetic field data are in agreement with what it was previously inferred in this section, i.e. during a fast cooldown thermal gradients decrease as the SC transition moves towards the top of the cavity, causing flux to get trapped in this position. On the other hand, if the cooldown happens slowly the flux is homogeneously trapped around the cavity wall.

\subsection{Physics behind the flux hole scenario}

After the SC transition the magnetic field lines can redistribute in three possible ways: i) completely outside the cavity, ii) escaping through the beam pipes, or iii) escaping across the cavity wall if pinning centers are present, when a non-efficient expulsion occurs.

When the magnetic field is applied axially to the cavity (Figure 5.13 (a)) the expulsion can be efficient whenever thermal gradients are large enough to allow so, because the flux lines that cross the cavity walls always have an easy path to follow when they are expelled during the SC transition.

Whereas, when the applied field is perpendicular to the cavity axis (Figure 
$5.13(\mathrm{~b}))$, the magnetic flux lines redistributed with the ii) and iii) mechanisms do not have any possibility to escape from the cavity inner volume except crossing the cavity walls. Assuming a sharp SC-NC interface, the magnetic flux lines concentrate in the normal-conducting region, at the top of the cavity. This region becomes smaller and smaller as the transition boundary advances, until the magnetic field is completely squeezed at the very top of the cavity equator. The top point of the cell is the last to be cooled below the transition temperature $T_{c}$, becoming a "flux hole" in the superconductor, from which the flux is not energetically favorable to escape, as the only way out would be via crossing the already superconducting regions.

The situation depicted implies that the geometry of the system could lead to incomplete Meissner effect, even though the thermal gradient across the cavity is high enough to provide efficient flux expulsion.

This may explain why the orthogonal magnetic field series always lead to poorer cavity performance than the axial ones. As verified also with magnetic field data, the orthogonal magnetic field is trapped on top of the cavity equator during fast cooldowns even when large thermal gradients are present, leading to temperature rising starting from medium RF field values.

\subsection{Conclusions}

This chapter presents the study of a superconducting single cell elliptical cavity that was horizontally cooled in presence of different magnetic field orientations.

The first important conclusion is that cooling cavities in the horizontal orientation results in the thermal gradient at the SC-NC phase front, which - differently from the vertical cavity orientation cooling - significantly decreases at the later stages of the cavity transition when the top of the cavity is approached. This reduce thermal gradient at the top leads to more trapped flux in that region, and therefore an increase 
in $\mathrm{RF}$ losses as it resides in the high surface magnetic field area. Cooldown procedure should therefore be sought in accelerators to ensure that a sufficient thermal gradient is maintained throughout the full cell profile, or that the final resting place of flux is not at a cavity equator.

The second important conclusion is that different field orientations may have a different impact on final performance; in particular, an orthogonal magnetic field may have a larger degrading impact for RF losses than an axial component for the same efficient cooldown procedure. Finally, an important new phenomena of heating at the top of the cavity has been observed in both the single cell and dressed nine cell studies, compatible with the "flux hole" scenario, where vertical field lines become encircled by superconducting regions and highly concentrated at the very top of the cavity. This can be harmful for cavity performance in an accelerator and could lead to both Q-factor and quench degradation. 


\section{CHAPTER 6 \\ COOLDOWN DYNAMICS: FAST VS SLOW}

\subsection{Introduction}

As mentioned in Chapter 5, fast cooldowns magnetic flux expulsion when large thermal gradients are generated along the cavity during the superconducting transition. On the other hand, slow cooldowns lead to fully trap the external magnetic field causing the increasing of losses.

Looking at the flux expulsion behavior of a cavity vertically cooled, the correlation between the flux trapping and the thermal gradient along the cavity appears clear looking at the graph of flux expulsion ratio $\left(B_{\mathrm{SC}} / B_{\mathrm{NC}}\right)$ versus the temperature difference $T_{1}-T_{2}$ (see Figure 6.1 ), where $B_{\mathrm{SC}}$ and $B_{\mathrm{NC}}$ are respectively the magnetic field after and before the superconducting transition, and $T_{1}$ is the temperature of the top iris of the cavity, measured when the temperature at the equator $\left(T_{2}\right)$ is equal to the critical temperature $T_{c}$. As discussed in the previous chapter, considering the cavity cooled in presence of axial magnetic field, $B_{\mathrm{SC}} / B_{\mathrm{NC}} \simeq 1.74$ in case of perfect Meissner effect and $B_{\mathrm{SC}} / B_{\mathrm{NC}} \simeq 1$ in case of complete flux trapping.

This phenomenon was first studied in Reference [54], in which the authors hypothesized two possible mechanisms that may explain the difference between fast and slow cooldown. The first mechanism takes into account that thermal gradient may generate a thermal force capable to depin trapped flux from the superconductor, allowing efficient magnetic flux expulsion. The second mechanism instead takes into account the possibility that during the fast cooldown the superconducting/normalconducting (SC-NC) transition progress sharply during all the process, while during the slow cooldown islands of normal-conducting material surrounded by superconducting material are generated. Therefore, during the slow-cooldown the magnetic 


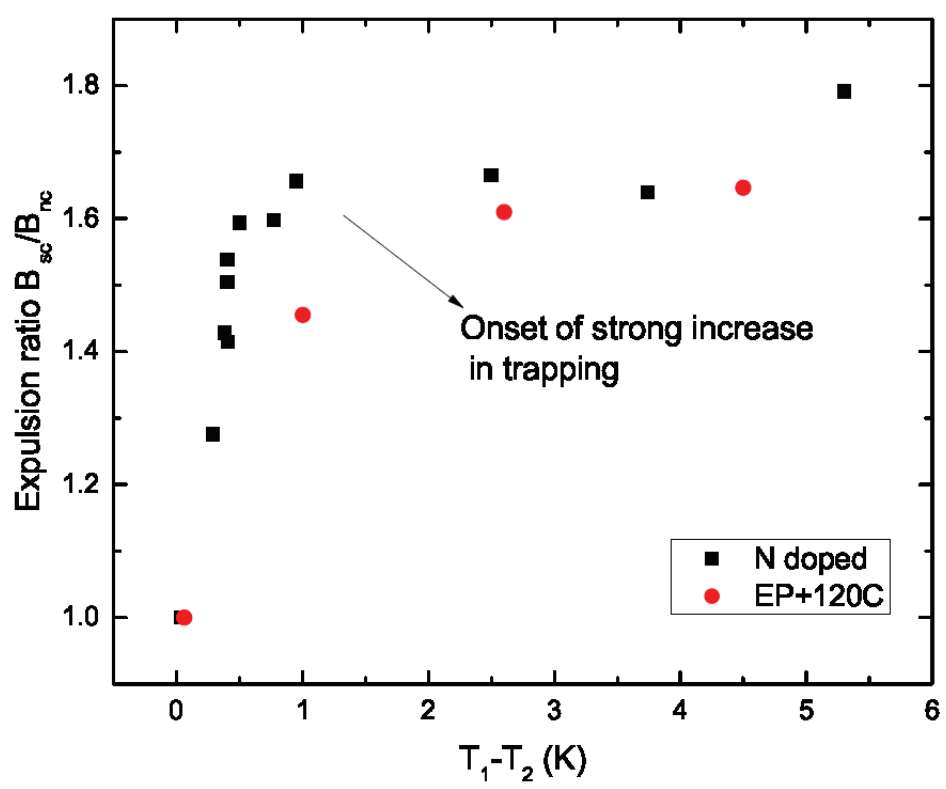

Figure 6.1. Ratio between the magnetic field after $B_{\mathrm{SC}}$ and before $B_{\mathrm{NC}}$ the superconducting transition as a function of the temperature difference $T_{1}-T_{2}$ [5].

field in the NC region cannot be expelled even after the complete SC transition of the cavity, since an energetically favorable path does not exists for the field to be expelled.

Recently, a theoretical model was proposed by T. Kubo [58] in order to explain the flux expulsion behavior as a function of the thermal gradient. This model considers that the mixed state covers a wider spatial range along the cavity during the cooldown when poor thermal gradients are present, compared to a scenario where thermal gradients are large. The presence of this wider range increases the probability of flux trapping. Under this assumption the number of trapped vortices are equal to:

$$
N_{\text {trap }}=A_{\mathrm{M}} B_{\text {ext }}\left(\frac{1}{\tilde{T}^{\prime}}+D_{\mathrm{M}}\right),
$$

where $A_{\mathrm{M}}$ is a material dependent parameter proportional to the number of pinning center, $B_{\text {ext }}$ is the external field, $\tilde{T}^{\prime}=T^{\prime} / T_{\mathrm{c}}$ is the thermal gradient $T^{\prime}$ normalized for the critical temperature $T_{\mathrm{c}}$ and $D_{\mathrm{M}}$ is another material dependent contribution.

As shown in [58] this model is capable of interpolating the data of Romanenko 
et al. [5] using $A_{M}$ and $D_{M}$ as free parameters of the interpolation.

The major limitation of this model is that for $\tilde{T} \rightarrow 0$, i.e for low values of thermal gradients, $N_{\text {trap }}$ diverges, implying that the amount of trapped field tends to infinity.

In this chapter a simple model is described in order to explain magnetic flux expulsion as a consequence of the thermodynamic force winning against the pinning force. In this model a Gaussian distribution of defects with different pinning forces is assumed in order to explain recent flux expulsion data, in which the level of flux expulsion is affected not only by the thermal gradient, but also by the micro-structure of the cavity material $[59,60]$.

In the second part of this chapter, the dynamics of fast and slow cooldown are studied by means of a T-map system capable to map the temperature around the cavity during the cooldown. This experiment pointed out that the complete flux trapping observed with slow cooldown may be due to normal-conducting islands from which the magnetic flux cannot be expelled even once the whole cavity is cooled below the critical temperature [61].

\subsection{Thermodynamic force acting on a vortex}

In order to understand the mechanism behind magnetic flux expulsion, a simple model that describes the vortex dynamics during the superconducting transition, governed by a thermodynamic force, is developed.

The Gibbs free energy density of a material in presence of magnetic field is defined as $g=f+B M$, where $f$ is the free energy density, $B$ the magnetic field induction and $M$ the magnetization. The magnetization of a superconductor in the Meissner state is defined by setting at zero the magnetic induction $B=\mu_{0}(H+M)=$ 0 , giving $M=-H$. Therefore, the Gibbs free energy density of a superconductor is 


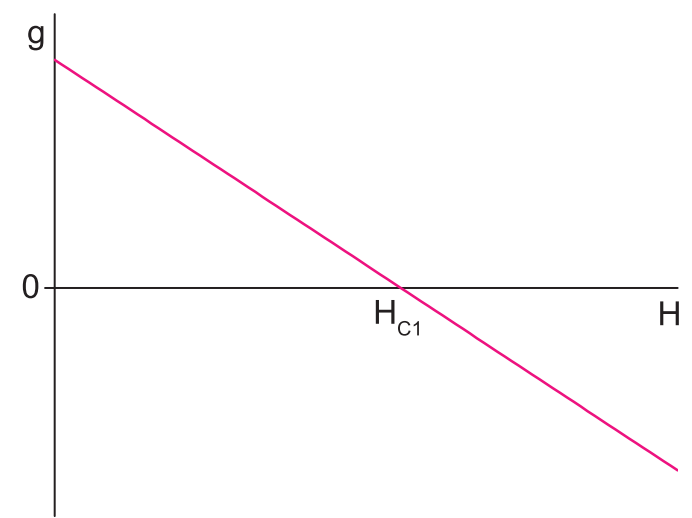

(a)

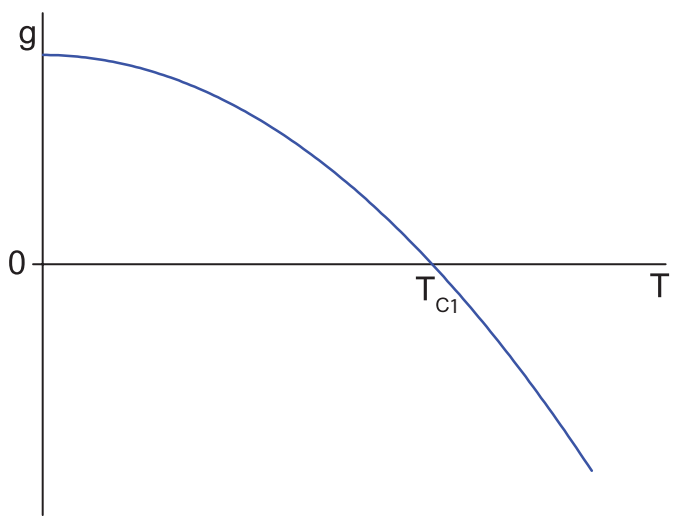

(b)

Figure 6.2. Gibbs free energy density $g$ as a function of the magnetic field $H$ (a) and as a function of temperature $T(b)$.

defined as:

$$
g=f-B H,
$$

where $f$ is the Helmholtz free energy density, defined as the areal vortex density $n$ times the energy per unit of length of a single vortex line $E$ : $f=n E$. Similarly, the magnetic induction can be defined as $B=n \Phi_{0}$, where $\Phi_{0}$ is the magnetic flux quanta. The Gibbs free energy can be rewritten as:

$$
g=n E-B H=B\left(H_{\mathrm{c} 1}-H\right)
$$

where $H_{\mathrm{c} 1}=E / \Phi_{0}$ is the lower critical field. In Figure 6.2 the Gibbs free energy density $g$ is plotted as a function of the field $H$. The field $H=H_{\mathrm{c} 1}$ represents the transition point from the Meissner $\left(H<H_{\mathrm{c} 1}\right)$ to the mixed $\left(H>H_{\mathrm{c} 1}\right)$ state. From Equation 6.3 , it is possible to notice that $g=0$ at the transition between the two states, $g>0$ in the Meissner state, and $g<0$ in the mixed state. This means that the Gibbs free energy is minimized when vortices are in the mixed state [36].

The temperature dependence in the Gibbs free energy is given by the temperature dependence of $H_{\mathrm{c} 1}$, resulting in:

$$
g=B\left[H_{\mathrm{c} 1}(0)\left(1-\left(\frac{T}{T_{\mathrm{c} 1}}\right)^{2}\right)-H\right],
$$


where $T_{\mathrm{c} 1}$ is the lower critical temperature, associated with the transition from the Meissner to the mixed state. In absence of magnetic field $T_{\mathrm{c} 1}=T_{\mathrm{c} 2}=T_{\mathrm{c}}$. Since during the cooldown of superconducting cavities the external magnetic field is actually very small, of the order of some milliGauss, it is possible to assume that $T_{\mathrm{c} 1} \simeq T_{\mathrm{c}}$. Considering an uni-dimensional thermal gradient, the force that drives the vortex movement can be defined as:

$$
f=-\frac{\partial g}{\partial x}=-\frac{\partial g}{\partial T} \frac{\partial T}{\partial x}
$$

where $\nabla T=\partial T / \partial x$ is the thermal gradient along the $x$ direction. From the derivative of Equation 6.4, the force per unit of volume results:

$$
f=\frac{2 B H_{\mathrm{c} 1}(0) T}{T_{\mathrm{c}}^{2}} \nabla T
$$

which defines that during the superconducting transition vortices move toward the direction of the thermal gradient, minimizing the Gibbs free energy.

During the superconducting transition, this thermodynamic force moves free vortices from the Meissner to the mixed state. Considering a fast cooldown of a cavity, the superconducting transition starts from the bottom and propagates through the top of the cavity. The same happens for the vortices which are continuously pushed up, toward the warm area of the cavity.

However, since some vortices may be pinned in defects in the material, the thermodynamic force has to be greater than the pinning force $f_{p}$, in order to be able to move the pinned vortices. The pinning force acts against the flux motion and it is generated by the interaction between flux lines and inhomogeneities or defects in the material. Historically, the pinning force was defined in terms of the minimum current density needed to depin vortices, i.e. the critical current density $J_{c}$. In this scenario the pinning force compensates the Lorentz force acting on a vortex and the pinning 
force per unit of volume can be defined as:

$$
f_{p}=\left|\bar{J}_{\mathrm{c}} \times n \bar{\Phi}_{0}\right|=J_{\mathrm{c}} B
$$

where $n=B / \Phi_{0}$ is the vortex density and $J_{\mathrm{c}}$ is the critical current density.

By equating Equation 6.6 to 6.7, it is possible to define the thermal gradient for which the two forces compensate each other, the so-called critical thermal gradient:

$$
\nabla T_{\mathrm{c}}=\frac{J_{\mathrm{c}} T_{\mathrm{c}}^{2}}{2 H_{\mathrm{c} 1}(0) T},
$$

which defines the minimal thermal gradient needed to depin a vortex subjected to pinning. For a certain superconductor, with $T_{\mathrm{c}}$ and $H_{\mathrm{c} 1}(0)$ fixed, the critical thermal gradient depends on the temperature and on the critical current density $J_{\mathrm{c}}$. Where $J_{\mathrm{c}}$ is an average value that takes into account the different nature and concentration of the defects present in the material.

Note also that to compare the model results with the experimental data, the thermal gradient $\nabla T_{\mathrm{c}}$ has to be convert in the temperature difference $\Delta T$ measured during the superconducting transition, which is the temperature difference along the cavity cell, from bottom iris to top iris (or along half cavity cell, from equator to upper iris). Therefore:

$$
\Delta T_{\mathrm{c}} \simeq \nabla T_{\mathrm{c}} \cdot l_{1 / 2 \mathrm{cell}} \quad \Delta T_{\mathrm{c}} \simeq \nabla T_{\mathrm{c}} \cdot l_{\text {cell }}
$$

where $l_{\text {cell }} \simeq 32 \mathrm{~cm}$ is the length of the cavity cell and $l_{1 / 2 \text { cell }} \simeq 16 \mathrm{~cm}$ is the length of half cell. Depending on how data are shown, it is necessary to use one or the other definition.

The critical current density is shown as a function of the critical temperature difference $\Delta T_{\mathrm{c}}$ in Figure 6.3, considering $T=T_{\mathrm{c}}=9.2 \mathrm{~K}$ and $B_{\mathrm{c} 1}(0)=\mu_{0} H_{\mathrm{c} 1}(0)=180$ $\mathrm{mT}$. From this figure it is possible to notice that, if complete flux expulsion is reached 


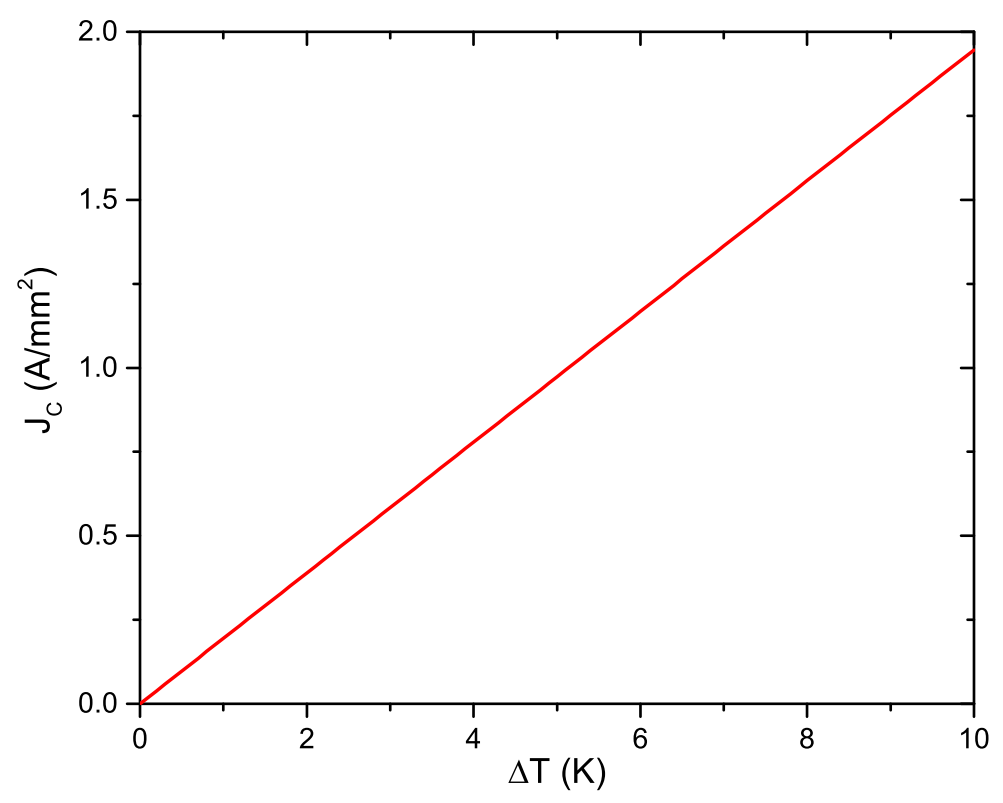

Figure 6.3. Critical current density as a function of the critical temperature difference $\Delta T_{\mathrm{c}}$.

with a temperature difference of $\Delta T=5 \mathrm{~K}$ along the cavity cell, the average critical current density has to be of the order of $J_{\mathrm{c}} \sim 1 \mathrm{~A} / \mathrm{mm}^{2}$, which seems to be a reasonable value for niobium near $T_{\mathrm{c}}[62]$.

\subsection{Statistical model of the flux expulsion}

It is reasonable to assume that in the superconducting material there is a statistical distribution of defects, where each defect is characterized by a certain pinning force. Therefore, this model assumes that the distribution of pinning centers can be described with a probability density function that defines the probability of expelling a vortex that has a pinning force $f_{\mathrm{p}, \mathrm{i}}$. The probability density function $(\mathrm{PDF})$ is centered around a most probable value of pinning force $f_{\mathrm{p}_{0}}$. Since the pinning force can be directly converted in critical current density, the probability of expelling vortices can also be described by the critical current $J_{\mathrm{c}_{\mathrm{i}}}$ associated with the pinning force $f_{\mathrm{p}, \mathrm{i}}$.

The critical thermal gradient is, in turn, directly proportional to $J_{\mathrm{c}}$ at a specific 


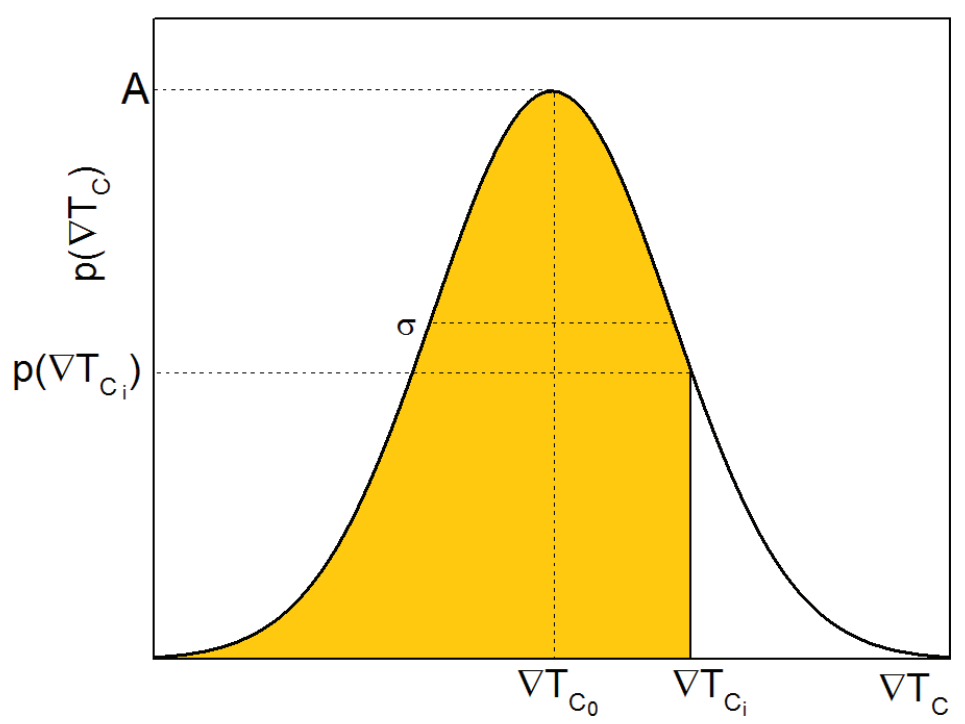

Figure 6.4. Gaussian probability density function as a function of the critical temperature difference $\Delta T_{\mathrm{c}}$. The orange area defines the probability that vortices are swiped out when the critical temperature difference $\Delta T_{\mathrm{C}_{\mathrm{i}}}$ is reached.

temperature, therefore the statistical distribution of $J_{\mathrm{c}}$ may be converted in $\nabla T_{\mathrm{c}}$ and then in $\Delta T_{\mathrm{c}}$. Assuming that the distribution of defects have a random nature, the probability that a vortex is pinned with a force proportional to the critical thermal gradient $\nabla T_{\mathrm{c}}$ may be described with a Gaussian probability density function. The Gaussian distribution's probability is defined as:

$$
P\left(\nabla T_{\mathrm{c}}\right)=\int_{0}^{\nabla T_{\mathrm{c}}} A e^{-\frac{\left(\nabla T_{\mathrm{c}}-\nabla T_{\mathrm{c}_{0}}\right)^{2}}{2 \sigma^{2}}} d \nabla T_{\mathrm{c}}
$$

where $A$ is the normalization constant, $\nabla T_{\mathrm{c}_{0}}$ is the centroid of the PDF and $\sigma^{2}$ is its variance. The normalization constant $A$ can be calculated by solving the following integral:

$$
P\left(\nabla T_{\mathrm{c}}\right)=\int_{0}^{\nabla T_{\mathrm{c}_{\max }}} A e^{-\frac{\left(\nabla T_{\mathrm{c}_{\mathrm{i}}}-\nabla T_{\mathrm{c}_{0}}\right)^{2}}{2 \sigma^{2}}} d \nabla T_{\mathrm{c}}=1,
$$

this integral assures that the area of the Guassian distribution is equal to 1 . This implies that, considering all the possible values of critical thermal gradient $\nabla T_{\mathrm{c}_{\mathrm{i}}}$, the probability of expelling vortices is maximum and equal to 1 . In order to solve thin 
integral numerically, the upper limit can not be set to infinite, so it is set at a value $\nabla T_{\mathrm{c}_{\max }}>>\nabla T_{\mathrm{c}_{0}}$

The normalization constant $A$ is therefore equal to:

$$
A=\frac{\sqrt{\frac{2}{\pi}}}{\sigma\left[\operatorname{erf}\left(\frac{\nabla T_{\mathrm{c}_{\max }}-\nabla T_{\mathrm{c}_{0}}}{\sqrt{2} \sigma}\right)+\operatorname{erf}\left(\frac{\nabla T_{\mathrm{c}_{0}}}{\sqrt{2} \sigma}\right)\right]} .
$$

In Figure 6.4 the probability density $p\left(\nabla T_{\mathrm{c}}\right)$ is shown as a function of the critical thermal gradient $\nabla T_{\mathrm{c}}$. The orange area under the curve defines the probability $P\left(\nabla T_{\mathrm{c}_{\mathrm{i}}}\right)$ that vortices are swiped out with a thermal gradient $\nabla T_{\mathrm{c}_{\mathrm{i}}}$. On the other hand, the white area defines the probability $1-P\left(\nabla T_{\mathrm{c}_{\mathrm{i}}}\right)$ of trapping vortices at the thermal gradient $\nabla T_{\mathrm{c}_{\mathrm{i}}}$.

It is possible now to define the density of trapped vortices, $n_{\text {trap }}$, as the probability to trap vortices, times the vortex density $n=B_{\mathrm{e}} / \Phi_{0}$ generated by applying an external magnetic field $B_{\mathrm{e}}$ :

$$
n_{\text {trap }}=\left[1-P\left(\nabla T_{\mathrm{c}_{\mathrm{i}}}\right)\right] n
$$

Knowing $n_{\text {trap }}$, the trapped magnetic field may be easily calculated as: $B_{\text {trap }}=$ $n_{\text {trap }} \Phi_{0}$

Therefore, using the definition of $P\left(\nabla T_{\mathrm{c}_{\mathrm{i}}}\right)$ in Equation 6.11, the total magnetic field trapped in the cavity results:

$$
B_{\text {trap }}=B_{\mathrm{e}}\left[1-\int_{0}^{\nabla T_{\mathrm{c}_{\mathrm{i}}}} A e^{-\frac{\left(\nabla T_{\mathrm{c}}-\nabla T_{\mathrm{c}_{0}}\right)^{2}}{2 \sigma^{2}}} d \nabla T_{\mathrm{c}}\right]
$$

where $\nabla T_{\mathrm{c}}$ is defined by Equation 6.8.

Experimental data of flux expulsion are usually represented as flux expulsion ratio $\left(B_{\mathrm{SC}} / B_{\mathrm{NC}}\right)$ as a function of the thermal gradient along the cavity, from equator to upper iris, or from bottom iris to upper iris. 
Therefore, in order to compare the statistical model with the experimental data, it is useful to convert the $B_{\text {trap }}$ in $B_{\mathrm{SC}} / B_{\mathrm{NC}}$. In order to perform this conversion, it is necessary to take into account that when $B_{\mathrm{SC}} / B_{\mathrm{NC}} \simeq 1.74$ the flux is completely expelled, while when $B_{\mathrm{SC}} / B_{\mathrm{NC}} \simeq 1$ the flux is completely trapped in the cavity walls (as defined in Chapter 5). Considering then a linear trend of the flux expulsion within $1<B_{\mathrm{SC}} / B_{\mathrm{NC}}<1.74$, the trapped flux can be defined as:

$$
B_{\text {trap }}=B_{\mathrm{NC}}\left[1-\frac{\frac{B_{\mathrm{SC}}}{B_{\mathrm{NC}}}-1}{0.74}\right],
$$

and solving this formula for $B_{\mathrm{SC}} / B_{\mathrm{NC}}$, the flux expulsion ratio can be calculated knowing the trapped field $B_{\text {trap }}$ and the external magnetic field when the cavity is still normal-conducting $B_{\mathrm{e}}=B_{\mathrm{NC}}$. In Figure 6.5, the percentage of trapped flux $\% B_{\text {trap }}$ as a function of the ratio $B_{\mathrm{SC}} / B_{\mathrm{NC}}$ is shown. The red line is calculated using Equation 6.15, while the light blue points are experimental data that confirms the linear trend. The $\% B_{\text {trap }}$ of the experimental data is calculated as:

$$
\% B_{\text {trap }}=\frac{R_{\mathrm{ff}}}{S} \cdot 100
$$

where $R_{\mathrm{f}}$ is the trapped flux surface resistance and $S$ the trapped flux sensitivity. The meaning of these parameters are well discussed in Chapter 7.

6.3.1 Single distribution of pinning centers. In Figure 6.6, the probability density $p\left(\nabla T_{\mathrm{c}}\right)$ is shown as a function of the critical thermal gradient, for three examples of PDF. The PDF shown with the red curve (a) has the same centroid $\nabla T_{\mathrm{c}_{0}}$ of the blue curve (b) but double standard deviation $\sigma$. The PDF shown with the green curve instead (c) has same $\sigma$ of the red curve but double value of $\Delta T_{\mathrm{c}_{0}}$. The legend shows the values of the centroids of the PDF converted in $J_{\text {c }}$.

Interesting is to see what is the effect of the different parameters of the PDF, on the flux expulsion behavior. In Figure 6.7, the flux expulsion ratio as a function of the temperature difference $\Delta T$ is shown for the three PDF shown in Figure 6.6. 


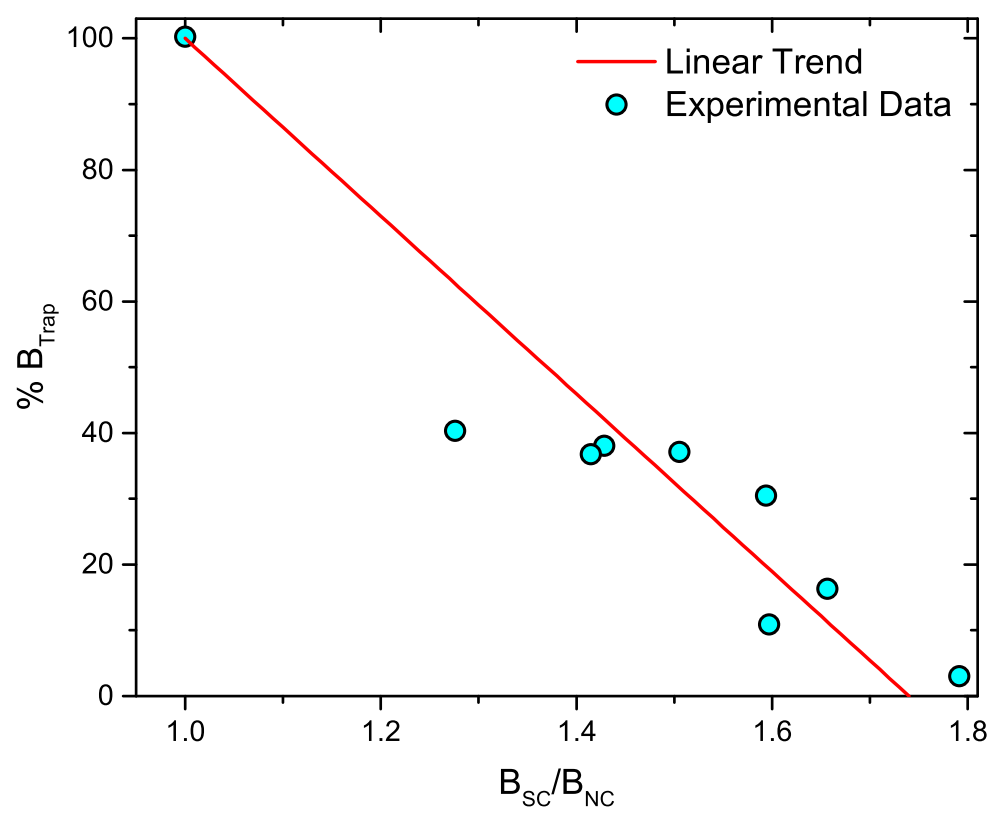

Figure 6.5. Linear trend of the percentage of trapped field $\left(B_{\text {trap }}\right)$ versus the flux expulsion ratio $B_{\mathrm{SC}} / B_{\mathrm{NC}}$.

It is possible to notice that when the standard deviation of the PDF is doubled (curve a vs b in Figure 6.7), this has a considerably large effect on the flux expulsion behavior, increasing the thermal gradient needed to reach complete flux expulsion. This effect seems to be less pronounced when the center value of the PDF is doubled (curve a vs c in Figure 6.7). In this case indeed the thermal gradient needed to reach complete flux expulsion remains unchanged. The slope of the flux expulsion ratio is instead lowered, indicating poorer flux expulsion behavior for low values of thermal gradients.

6.3.2 Double distribution of pinning centers. Depending on the material, more than one distribution of pinning centers may be present. Each group of defects as dislocations, grain boundaries, precipitates, etc., defines a certain probability to expel vortices with a pinning force proportional to a specific $J_{\mathrm{c}_{\mathrm{i}}}$ and $\nabla T_{\mathrm{c}_{\mathrm{i}}}$.

Therefore, the model take into account the presence of two distributions of pinning centers through a double peaked probability density function. This time the 


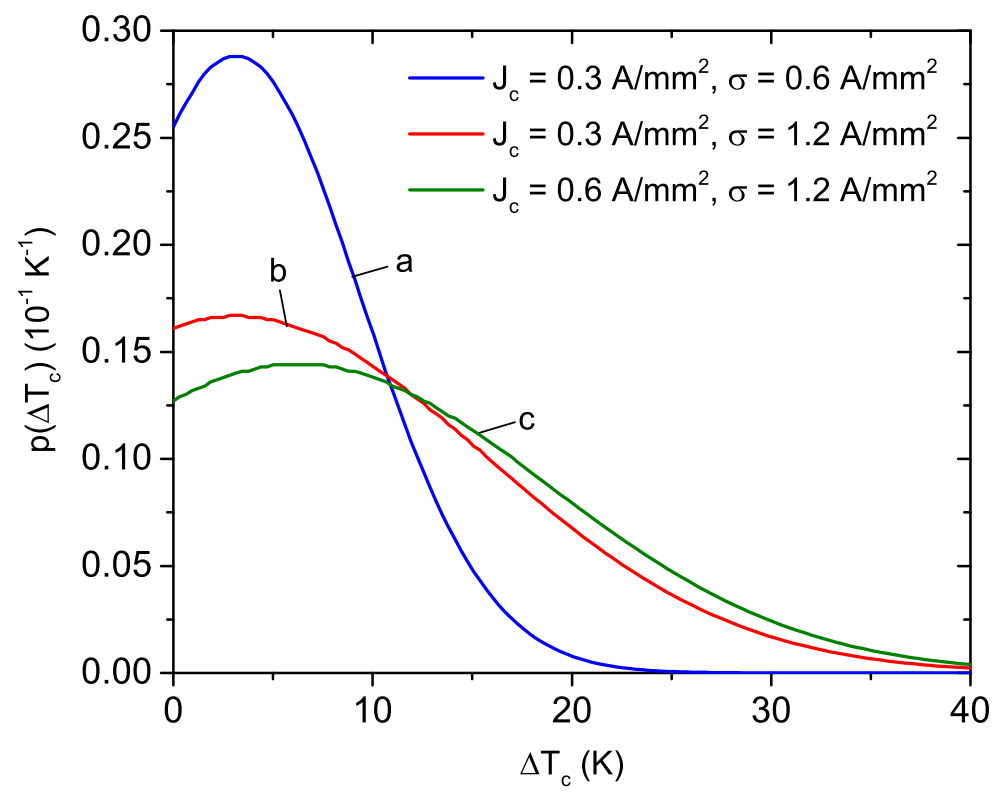

Figure 6.6. Probability density of expelling vortices as a function of the critical temperature difference for three example of probability density functions.

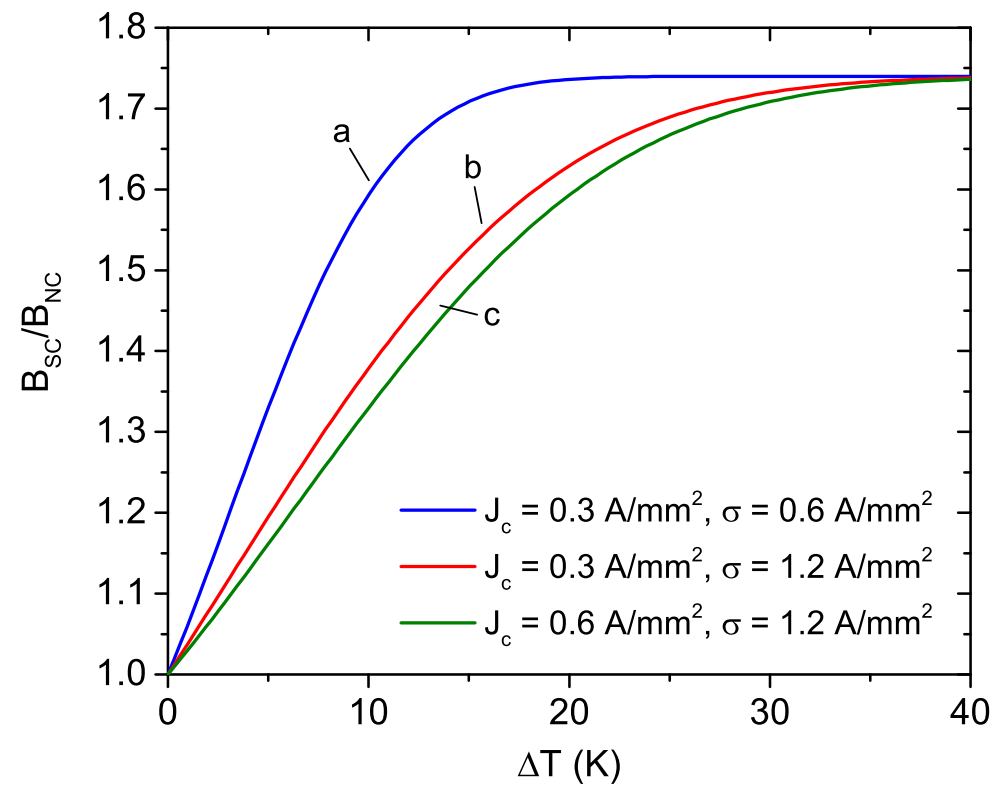

Figure 6.7. Flux expulsion ratio as a function of the temperature difference for the three example of probability density functions described in Figure 6.6. 
normalization is made taking into account both the distributions in order to consider that the complete flux expulsion is achieved only when vortices are flushed out from both distributions of pinning centers.

In order to understand how the presence of the second distribution modifies the flux expulsion property, in Figure 6.8 the probability density function is shown, for four example of pinning centers distributions, as a function of the temperature difference $\Delta T_{\mathrm{c}}$. In Figure 6.9, are shown the correspondent curves of flux expulsion ratio as a function of the temperature difference.

The four examples have the same parameters for the first peak of the PDF and different parameters for the second peak. Looking at the red curve (b), its second peak has double standard deviation $\sigma$ than the one of the blue curve (a). The green curve (c) has instead same $\sigma$ but double $\Delta T_{\mathrm{c}_{0}}$ compared to the red curve (b). The orange curve $(\mathrm{d})$ has the same center value $\Delta T_{\mathrm{c}_{0}}$ but double $\sigma$ compared to the green curve (c).

From Figure 6.9 it is possible to see that, when $\sigma$ of the second peak is doubled, once $\Delta T$ reaches the value of the maximum critical thermal gradient of the first peak, the first plateau of the flux expulsion ratio appears at lower values of $B_{\mathrm{SC}} / B_{\mathrm{NC}}$ (comparing the red curve with the blue curve and the orange curve with the green curve of Figure 6.9). This happens because the percentage of vortices flushed out from the first distribution of pinning centers is lowered, since a bigger fraction is now part of the second distribution. When instead the centroid of the peak is doubled, the first plateau of the flux expulsion ratio last for higher values of $\Delta T$. It is indeed necessary to reach larger thermal gradients in order to start to expel vortices pinned to the second distribution of pinning centers.

6.3.3 Comparison with experimental data. In order to verify this model, the 


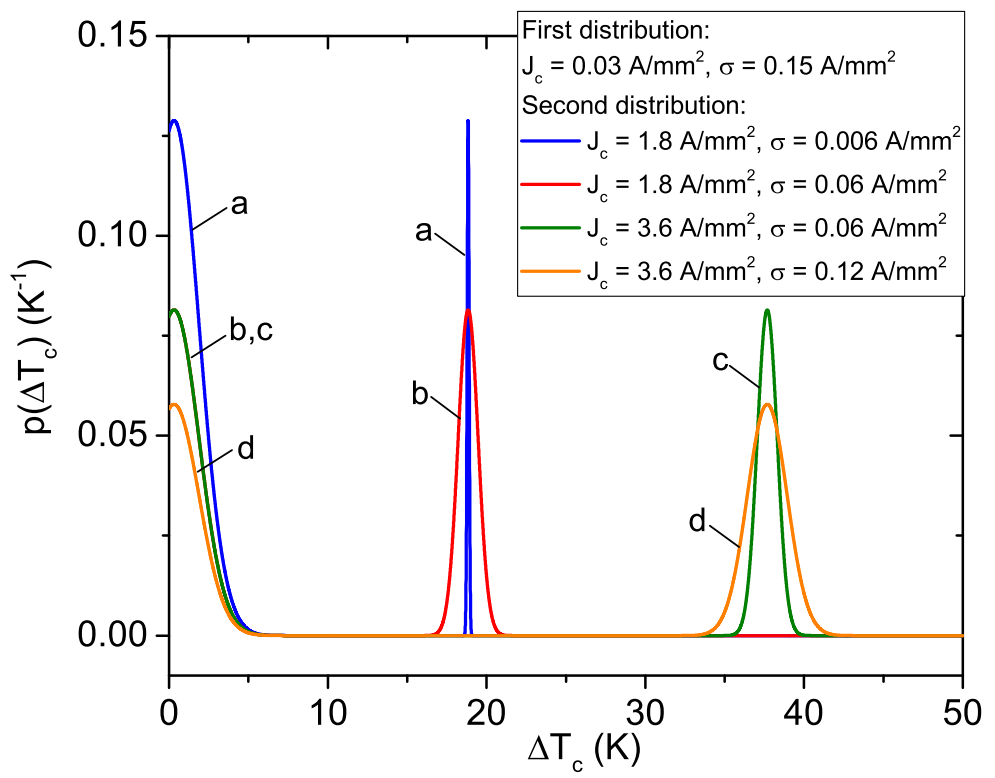

Figure 6.8. Probability density of expelling vortices as a function of the critical temperature difference along the cavity cell, for four example of probability density functions.

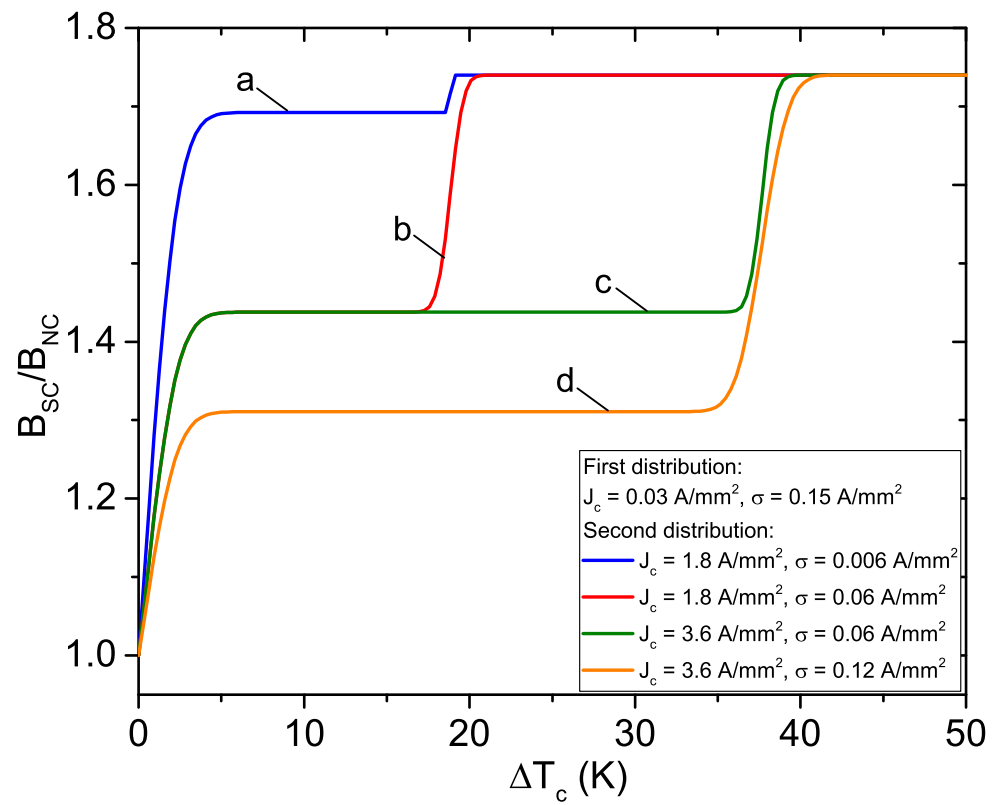

Figure 6.9. Flux expulsion ratio as a function of the temperature difference along the cavity cell, for the four example of probability density functions described in Figure 6.6. 
experimental data show in the References $[59,5]$ are interpolated.

In Figure 6.10, the flux expulsion data of the cavity AES011 studied in Reference [5] is shown and interpolated with the statistical model. In Figure 6.11, the same is shown for the cavity CBMM studied in Reference [59]. The blue curves are the results of the statistical model, in which the parameters $\nabla T_{0}$ and $\sigma$ are optimized in order to make the model adhere to the experimental points. The red curve show instead the respective probability density function.

Both these cavities show good flux expulsion behavior, and the cavity AES011 is capable to reach the ideal value of $B_{\mathrm{SC}} / B_{\mathrm{NC}}=1.74$. For the cavity CBMM the flux expulsion ratio saturates around $B_{\mathrm{SC}} / B_{\mathrm{NC}} \simeq 1.7$, therefore for this cavity the ration $B_{\mathrm{SC}} / B_{\mathrm{NC}}=1.7$ was considered as indicating complete flux expulsion instead of $B_{\mathrm{SC}} / B_{\mathrm{NC}}=1.74$.

The parameters of the Gaussian probability density function are summarized in Table 6.1. The PDF of CBMM has larger values of both $\Delta T_{0}$ and $\sigma_{\Delta \mathrm{T}_{0}}$ than AES011, in agreement with the fact that larger thermal gradients are needed to flush out vortices. Note that the temperature difference $\Delta T$ of AES011 in Figure 6.10 is defined along half cell, while the one of CBMM in Figure 6.11 is defined along the entire cell (as all the other cavities).

As can be seen in Figures 6.10 and 6.11, the Gaussian PDF start with a finite value for $\Delta T=0$. This is necessary in order to take into account the finite probability that some vortices may not be pinned.

Using this single distribution of pinning centers, it is not possible to describe a scenario where the cavity is capable to expel flux only until a certain level. This is the case of some of the cavities studied in Reference [59] for which flux expulsion is not anymore improved by increasing the temperature difference along the cavity cell 


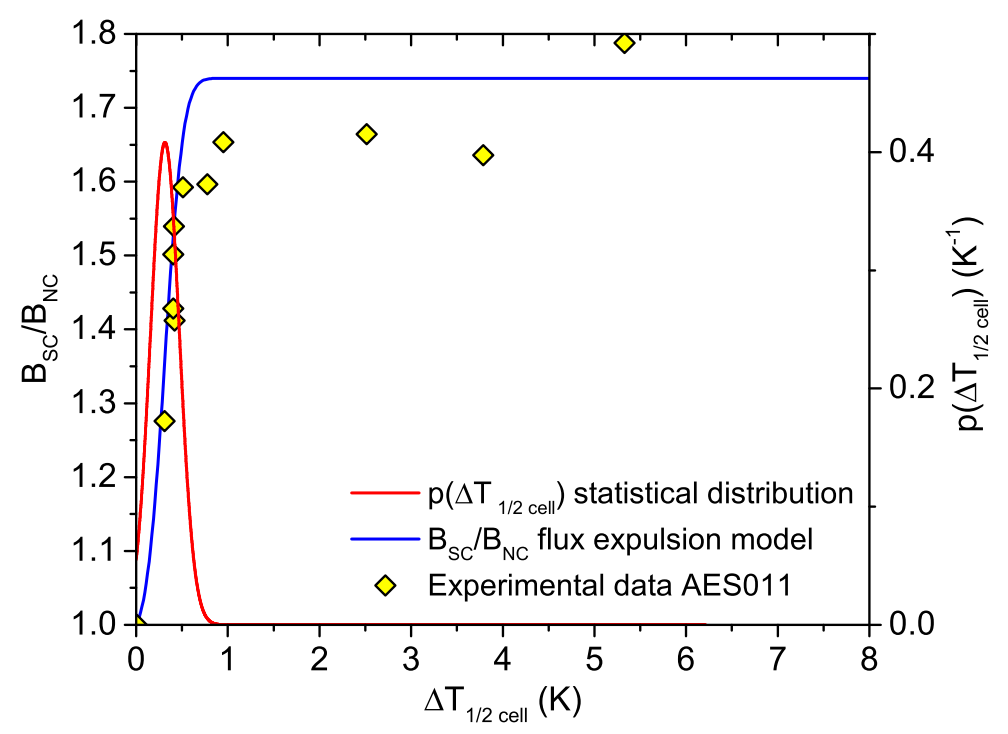

Figure 6.10. Comparison between the experimental values (yellow diamonds) and the simulated curve (blue curve) of the flux expulsion behavior of the cavity AES011. The red curve shows the probability density function used to generate the simulated curve.

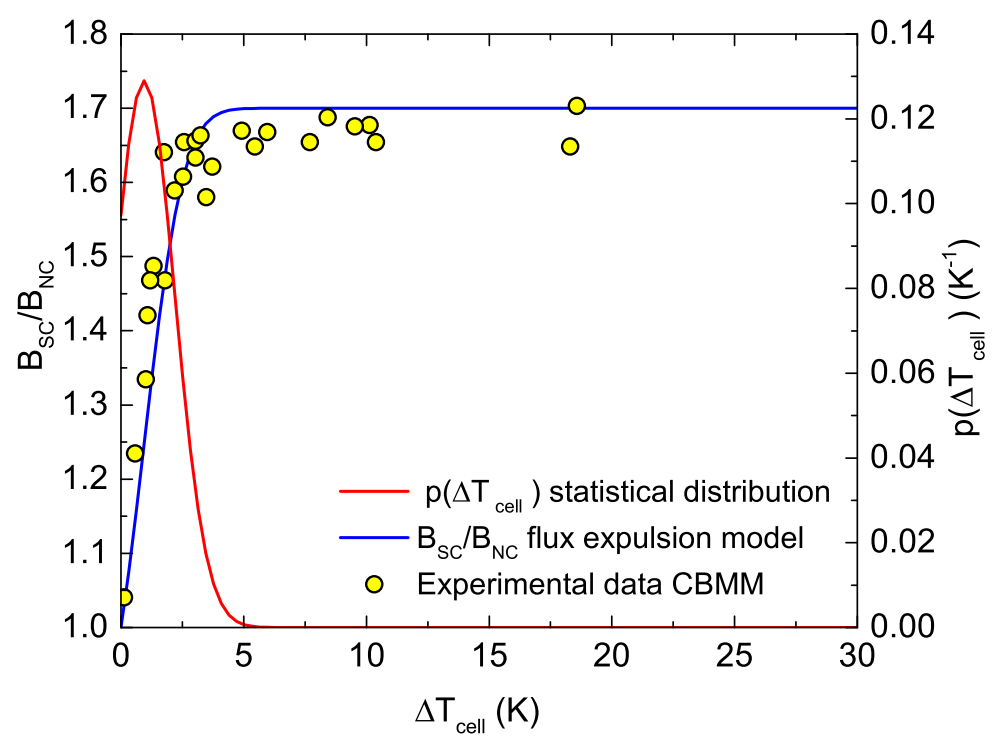

Figure 6.11. Comparison between the experimental values (yellow dots) and the simulated curve (blue curve) of the flux expulsion behavior of the cavity CBMM. The red curve shows the probability density function used to generate the simulated curve. 
Table 6.1. Summary of the parameters of the Gaussian probability density functions used to describe flux expulsion data of AES011 and CBMM.

\begin{tabular}{ccccc}
\hline \hline Cavity Name & $\Delta T_{0}(\mathrm{~K} / \mathrm{m})$ & $\sigma_{\Delta T_{0}}(\mathrm{~K} / \mathrm{m})$ & $J_{\mathrm{c}}\left(\mathrm{A} / \mathrm{mm}^{2}\right)$ & $\sigma_{\mathrm{J}_{\mathrm{c}}}\left(\mathrm{A} / \mathrm{mm}^{2}\right)$ \\
\hline AES011 & 2 & 1 & 0.06 & 0.03 \\
CBMM & 3 & 4 & 0.09 & 0.12 \\
\hline
\end{tabular}

during the cooldown.

This behavior may be interpreted as consequence of the presence of different distributions of pinning centers with larger average pinning force and therefore larger $\Delta T_{0}$. In this case, in order to achieve the complete flux expulsion, it is necessary to reach the critical thermal gradient of all the distributions.

A double peaked PDF is taken into account in order to describe the experimental data. The first peak is defined as the one with the lowest $\Delta T_{\mathrm{c}_{0}}$, which is indicated as $\Delta T_{\mathrm{c}_{0}}^{1} . \Delta T_{\mathrm{c}_{0}}$ of the second peak is indicated as $\Delta T_{\mathrm{c}_{0}}^{2}$ instead. The same notation is used to indicate the different values of $J_{\mathrm{c}_{0}}$ and $\sigma$.

In Figure 6.12 the simulations are compared with the flux expulsion data of the cavities CBMM and ACC002 show in Reference [59]. It is clear that in both cases, the model well interpolates the experimental data. The parameters set in the simulation are shown in Table 6.2.

Interpolating the data of the cavity CBMM using the double peaked PDF, it is clear that this cavity reached only $B_{\mathrm{SC}} / B_{\mathrm{NC}}=1.7$, because the thermal gradients reached during the cooldowns were actually lower than the critical thermal gradients needed to depin vortices from the second distribution of defects.

Comparing the parameters needed to simulate the flux expulsion behavior of CBMM with $\mathrm{ACC002}$, it is possible to notice that ACC002 needs larger thermal gradient than CBMM in order to reach the first plateau. This is due to both larger 


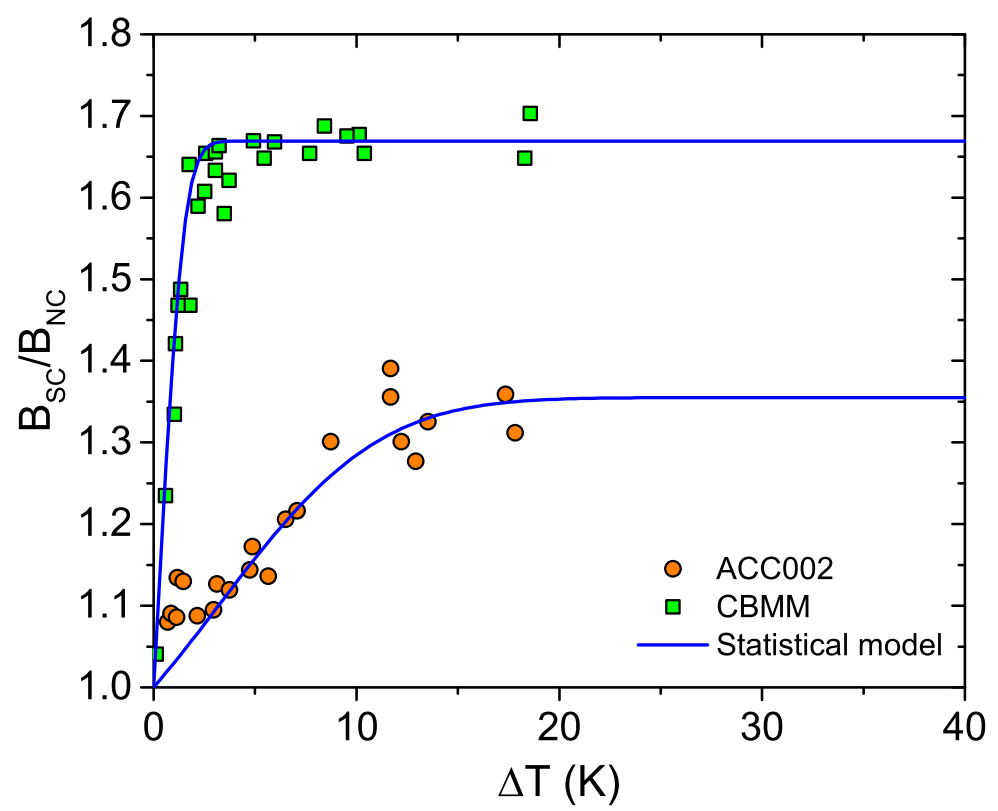

Figure 6.12. Comparison between the experimental values (green squares and orange circles) and the simulated curves (blue curve) of the flux expulsion behavior of the cavities CBMM and ACC002. The simulation is done taking into account a double distribution of pinning centers.

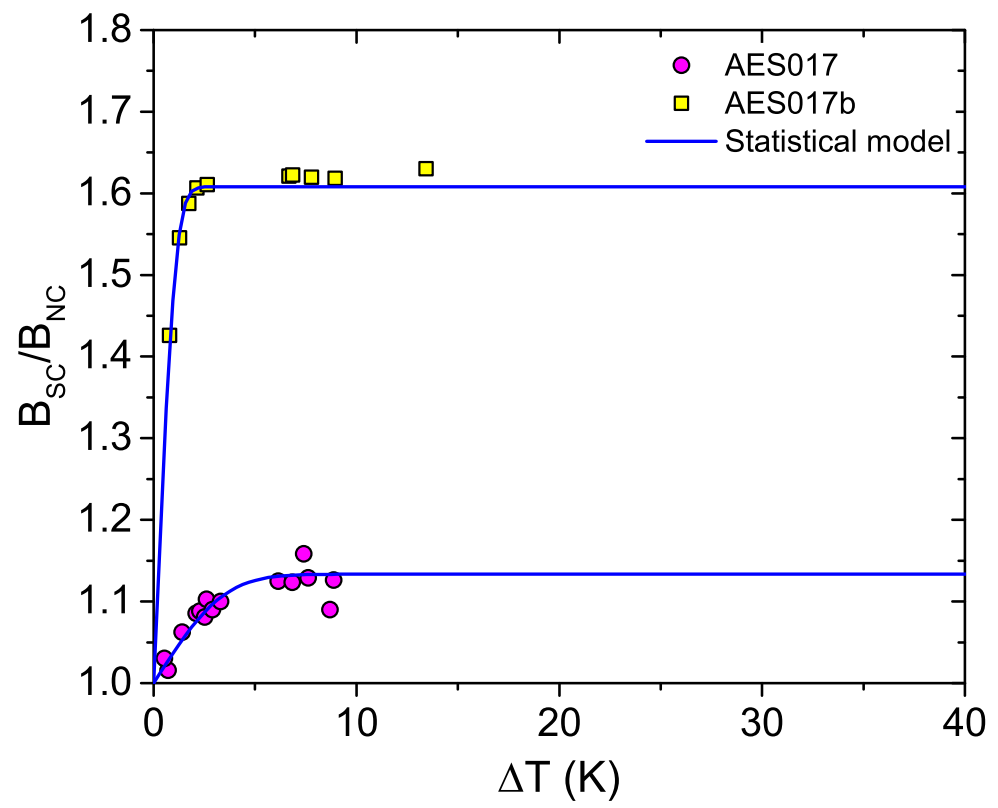

Figure 6.13. Comparison between the experimental values (pink circles and yellow squares) and the simulated curves (blue curve) of the flux expulsion behavior of the cavities AES017 and AES017b. The simulation is done taking into account a double distribution of pinning centers. 
Table 6.2. Summary of the parameters of the double peaked probability density function used to describe flux expulsion data of the cavities ACC002, CBMM, AES017 and AES017b.

\begin{tabular}{|c|c|c|c|c|c|c|c|c|}
\hline \multirow{2}{*}{ Cavity Name } & \multicolumn{4}{|c|}{ I distribution } & \multicolumn{4}{|c|}{ II distribution } \\
\hline & $\Delta T_{\mathrm{c}_{0}}^{1}(\mathrm{~K} / \mathrm{m})$ & $\sigma_{\Delta T_{\mathrm{c}_{0}}}^{1}(\mathrm{~K} / \mathrm{m})$ & $J_{\mathrm{c}_{0}}^{1}\left(\mathrm{~A} / \mathrm{mm}^{2}\right)$ & $\sigma_{J_{c_{0}}}^{1}\left(\mathrm{~A} / \mathrm{mm}^{2}\right)$ & $\Delta T_{\mathrm{c}_{0}}^{2}(\mathrm{~K} / \mathrm{m})$ & $\sigma_{\Delta T_{\mathrm{c}_{0}}}^{2}(\mathrm{~K} / \mathrm{m})$ & $J_{\mathrm{c}_{0}}^{2}\left(\mathrm{~A} / \mathrm{mm}^{2}\right)$ & $\sigma_{\mathrm{J}_{\mathrm{c}_{0}}}^{2}\left(\mathrm{~A} / \mathrm{mm}^{2}\right)$ \\
\hline ACC002 & 12 & 13 & 0.36 & 0.39 & 80 & 13 & 2.4 & 0.39 \\
\hline CBMM & 1 & 3 & 0.03 & 0.09 & 75 & 0.2 & 2.26 & 0.006 \\
\hline AES017 & 1 & 6 & 0.03 & 0.18 & 75 & 17 & 2.26 & 0.5 \\
\hline AES017b & 1 & 2 & 0.03 & 0.06 & 75 & 0.28 & 2.26 & 0.008 \\
\hline
\end{tabular}


$\Delta T_{\mathrm{c}_{0}}^{1}$ and $\sigma_{\Delta T_{\mathrm{c}_{0}}}$. The ratio between the two peaks area plays instead a central role in determining the value of $B_{\mathrm{SC}} / B_{\mathrm{NC}}$ of the plateau. The smaller value of ACC002 is given by the larger area of the second peak compared to the first one.

In Reference [59], it is also shown a case in which a cavity with poor flux expulsion property, become capable to efficiently expel magnetic flux after a heat treatment of one hour at $1000{ }^{\circ} \mathrm{C}$.

In Figure 6.13 the simulations are compared with the flux expulsion data of such cavity before (AES017) and after the high temperature baking (AES017b). Also in this case the simulations are capable to well describe the flux expulsion behaviors. The simulations are done considering the same $\Delta T_{\mathrm{c}_{0}}^{1}$ and $\Delta T_{\mathrm{c}_{0}}^{2}$ for both the double peaked PDF of AES017 and AES017b (see Table 6.2). The only parameters that are different between the two double peaked PDF are the two standard deviations $\sigma^{1}$ and $\sigma^{2}$. The $\sigma$ of both PDF are considerably narrowed after the baking treatment. The differences between the simulated distributions can be better seen in Figure 6.14.

It is possible to think that the high temperature baking randomly dissolve defects, without changing their own pinning force. This results in a narrower PDF, which is centered around the same peak centroid as before the baking treatment.

In order to better visualize the problem, let us assume an arbitrary initial number of defects equal to $N=20 \times 10^{7}$, distributed over a double distribution of pinning center with pinning force proportional to the critical thermal difference along the cavity $\Delta T_{\mathrm{c}}$, as shown in Figure 6.16 (a) by the blue histogram. From this distribution it is possible to calculate the probability density $p\left(\Delta T_{\mathrm{i}}\right)$ of swiping vortices out of the superconductor at $\Delta T_{\mathrm{c}_{\mathrm{i}}}$ :

$$
p\left(\Delta T_{\mathrm{c}_{\mathrm{i}}}\right)=\frac{n\left(\Delta T_{\mathrm{C}_{\mathrm{i}}}\right) / N}{w},
$$

where $w$ is the histogram's bin width $(w=2 \mathrm{~K})$. The probability density function 


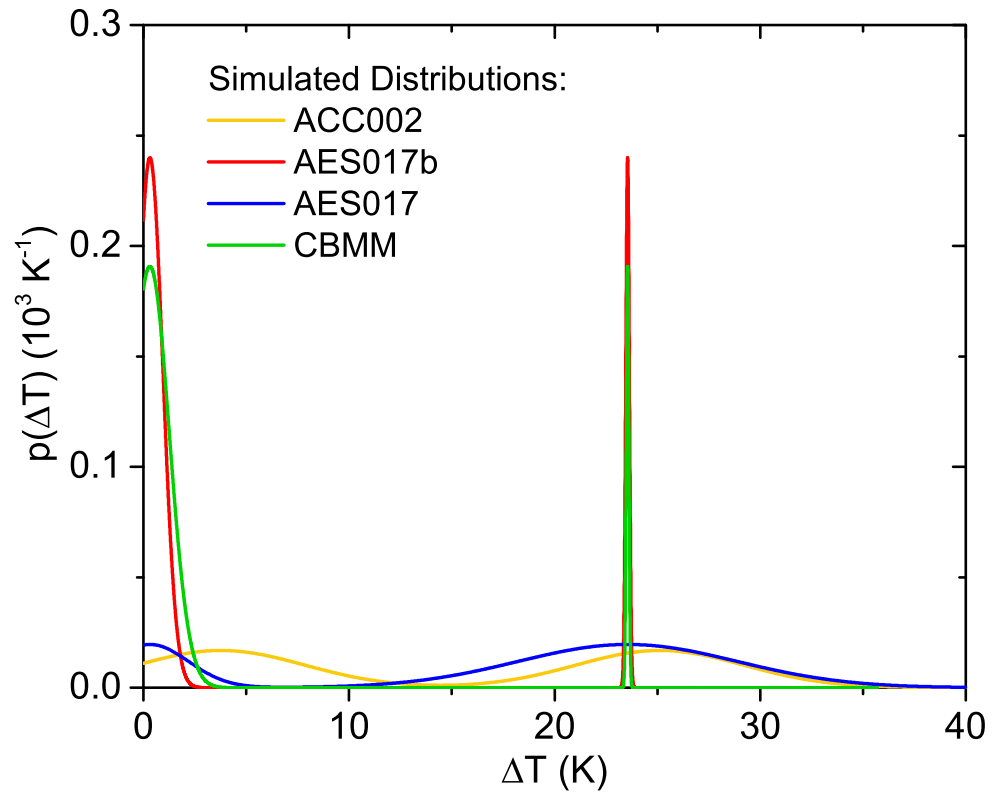

Figure 6.14. Double peaked probability density functions used to generate the flux expulsion curves of the studied cavities.

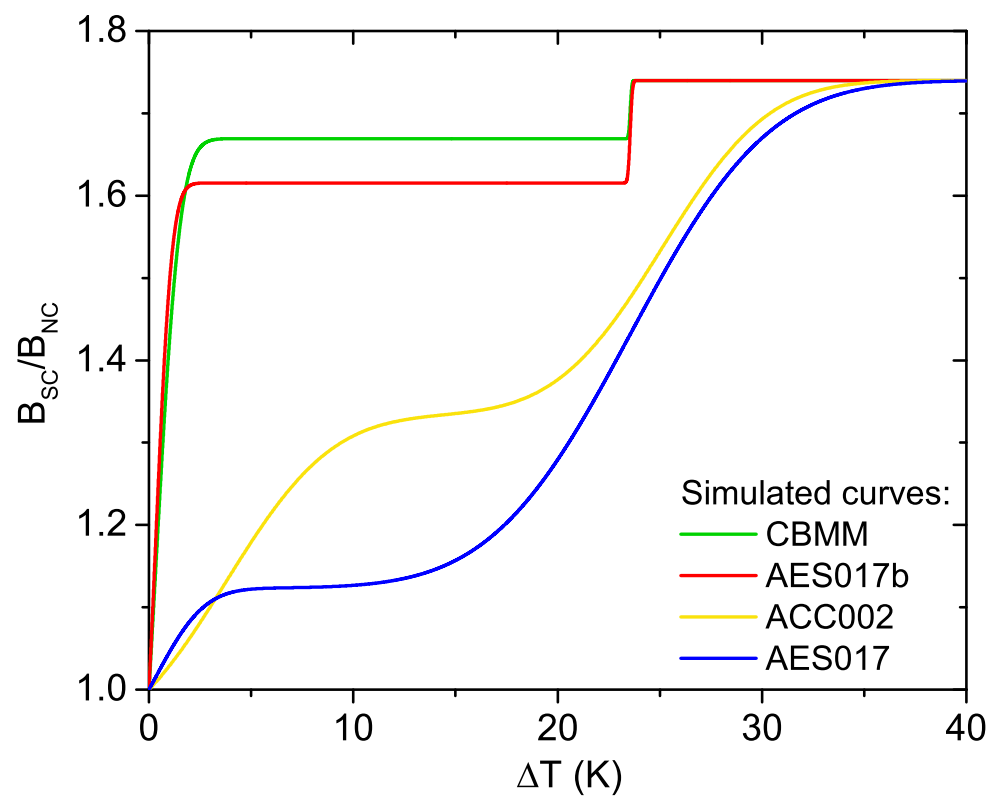

Figure 6.15. Modeled flux expulsion behaviors of the studied cavities. 


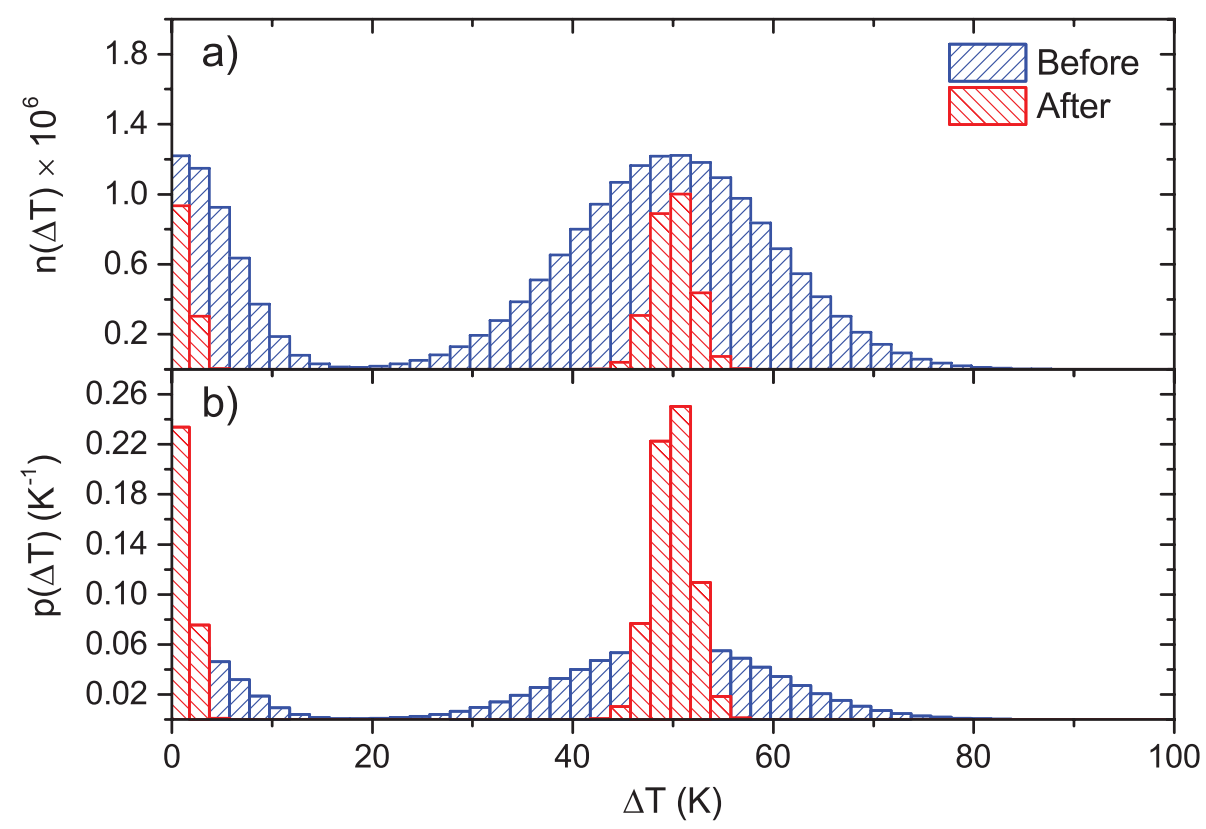

Figure 6.16. Histogram representation of the pinning centers distribution (a) and probability density of expelling vortices (b) before and after the high temperature baking.

calculated using Equation 6.17 is reported with the blue histogram in Figure 6.16 (b).

Now, assuming that after the high temperature baking, the total number of pinning centers $N$ decreased to one fifth of the initial value $\left(4 \times 10^{7}\right)$, the height and standard deviation of the distributions change accordingly. The distribution centroids remain instead fixed since not affected by the thermal treatment. In Figure 6.16 (a), the red histogram shows how the distribution of of pinning centers change after the baking under these assumptions.

In Figure 6.16 (b), the red histogram shown how the probability density $p(\Delta T)$ calculated with Equation 6.17, change after the baking treatment. Since the pinning center distributions are now narrower, the probability density function narrows accordingly and increases also its height in order to maintain the total probability equal to 1 . 


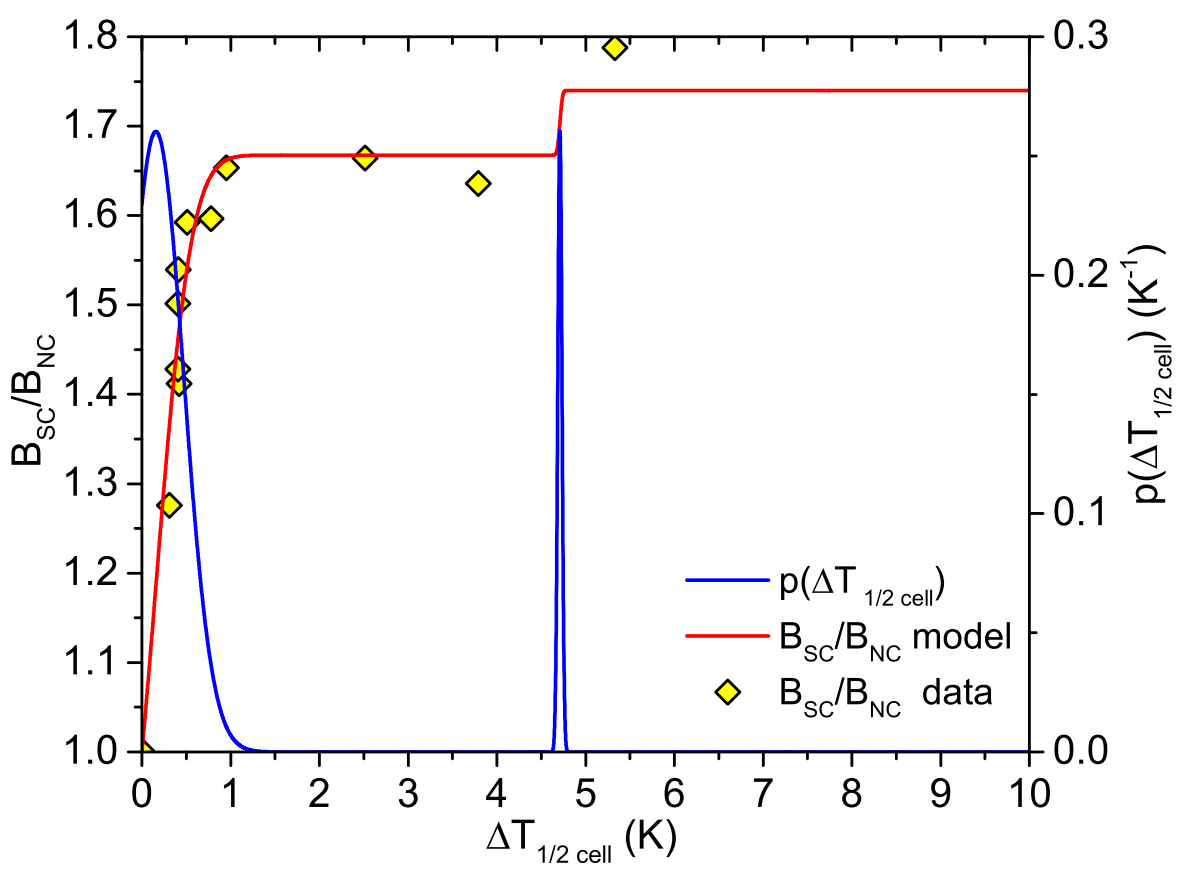

Figure 6.17. Comparison between experimental (yellow diamonds) and simulated flux expulsion data (blue curve) of the cavity AES011 using a double peaked probability density function.

In Figure 6.15 are show the complete flux expulsion curves simulated for the cavities CBMM, ACC002, AES017 and AES017b. As expected the thermal gradient needed in order to reach the complete flux expulsion are too large to be achieved during the cooldown. From the simulation, for all the four cavities, in order to reach $B_{\mathrm{SC}} / B_{\mathrm{NC}}=1.74, \Delta T$ of about $90 \mathrm{~K}$ are needed along the cavity cell.

Considering again cavity AES011, this cavity is the only one capable to reach $B_{\mathrm{SC}} / B_{\mathrm{NC}}=1.74$, as can be seen from Figure 6.10. However, from this graph it is possible to notice that only the point with $\Delta T_{1 / 2 \text { cell }}=5.5 \mathrm{~K}$ reaches this situation. Therefore, it may be possible that even this cavity has to be described by a double distribution of defects. The result obtained using a double distribution in shown in Figure 6.17. The model is capable to well simulate the flux expulsion behavior by using the following parameters: $J_{\mathrm{c}_{0}}^{1}=0.03 \mathrm{~A} / \mathrm{mm}^{2}, \sigma^{1}=0.06 \mathrm{~A} / \mathrm{mm}^{2}, J_{\mathrm{c}_{0}}^{2}=0.9$ $\mathrm{A} / \mathrm{mm}^{2}, \sigma^{2}=0.005 \mathrm{~A} / \mathrm{mm}^{2}$. 


\subsection{Cooldown dynamics recorded via T-map}

Flushing out of vortices due to the thermodynamic force is a possible mechanism that may explain both the intermediate and the complete magnetic flux expulsion behavior of superconducting cavities during their cooldown. However, in the rest part of this chapter the flux expulsion dynamics during fast and slow cooldown are analyzed in order to understand if their differences may play an important role too in determining the magnetic flux expulsion behavior.

When a slow cooldown is performed, the nucleation of the superconducting phase may be different compared to the one during the fast cooldown.

In order to verify whether the nucleation is really different in these two cases, the NC-SC transition dynamics is recorder by mapping the temperature all around the cavity during both a fast and a slow cooldown.

6.4.1 Experimental set-up. The cavity was instrumented with a T-map (Figure 6.18), an advanced diagnostic technique which allows to measure and map the temperature all around the cavity [63]. The FNAL T-map system consist on 570 thermometers installed on 36 boards that are assembled around the cavity every 10 degrees each. Every board counts 16 thermometers. In the experiments discuss in this chapter the T-map system is used for two different reasons: 1) to detect the temperature all around the cavity during the cooldown below its critical temperature and 2) to measure the temperature around the cavity during the RF measurement.

6.4.2 Data analysis and discussion. In order to clarify the difference between the dynamic of slow and fast cooldown, the T-map system is used to detect the temperature all around the cavity during both types of cooling.

In Figure 6.19 (a) is shown an example of a T-map image. The image illustrates that the white area corresponds to the superconducting region, with $T<9.25 \mathrm{~K}$, 


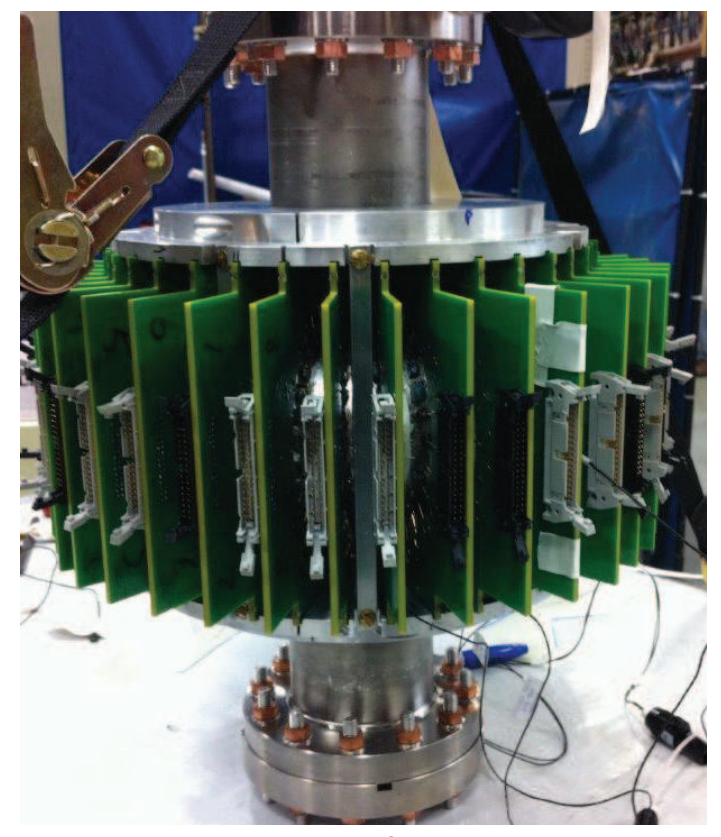

a)

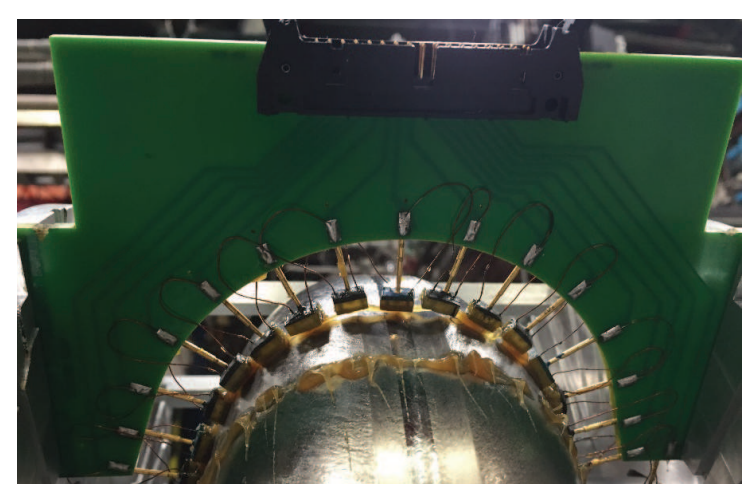

b)

Figure 6.18. Picture of: (a) the T-map system mounted on a single-cell cavity, (b) a single board of the T-map system mounted on the cavity.

while the colorful area corresponds to the normal-conducting region with temperature $T>9.25 \mathrm{~K}$ indicated by the color legend. In Figure 6.19 are also indicated the positions of both the bottom and the top of the cavity equator, which corresponds to the board number 13 and 31, respectively. Moreover, the temperature of the equatorial region of the cavity is given by the thermometer number 8 . This can be better seen from the sketch in Figure 6.19 (b) in which a scheme of the cavity is shown with one line of thermometers (board number 13).

The T-maps acquired during the fast cooldown are reported in Figures 6.20, 6.21 and 6.22. The Figures $6.20(\mathrm{a}),(\mathrm{b})$ and (c) shows the moments just before the starting of the superconducting transition. Figure 6.20 (d) capture the beginning of the $\mathrm{SC}$ transition, that starts from the bottom of the cavity equator, when the top is instead still at $T \sim 15 \mathrm{~K}$. From the successive images it is possible to notice than the interface between the normal-conducting and the superconducting propagates really 


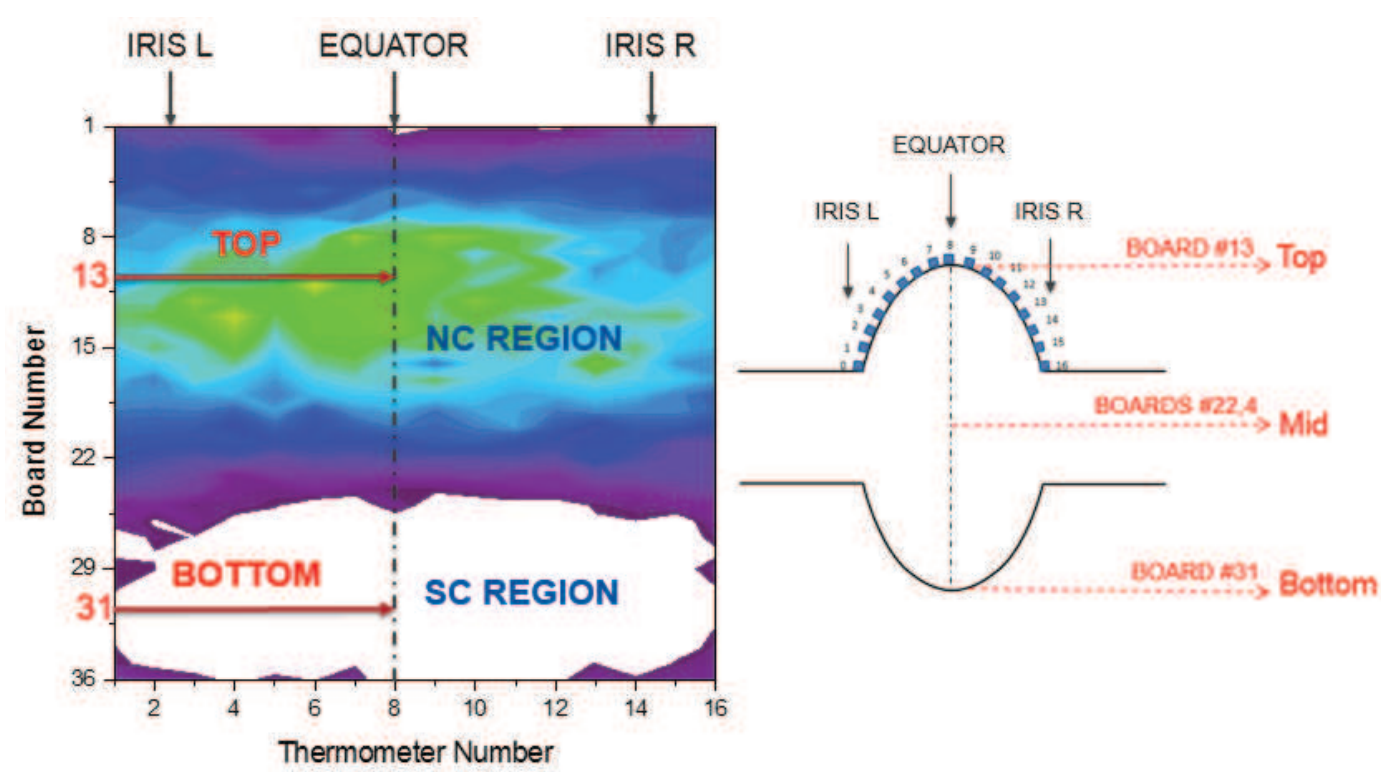

(a)

(b)

Figure 6.19. (a) Sketch of a T-map image in which the temperature of the top and the bottom of the cavity equator are pointed out. The image also shows that the equator temperature is recorded with the thermometer number 8 . The white region of the T-map indicates a superconducting area with $T<T_{c}$, while the colored region indicates the normal-conducting area with $T>T_{c}$. (b) Scheme of a horizontal single-cell cavity in which the top, mid and bottom positions are indicated with the correspondent T-map board number. The scheme also shows how the 16 thermometers of one board are arranged around the cavity cell.

sharply from the bottom to the top of the cavity (Figures 6.21 (c)-(f) and Figures 6.22 (a) and (b)). Only at the end of the cooling the interface becomes diffuse and some islands of normal-conducting phase appears at the top of the cavity (Figures $6.22(\mathrm{c})$ and $(\mathrm{d}))$.

The dynamic of the slow cooldown is shown in Figures 6.23, 6.24, 6.25, 6.26, 6.27 and 6.28. It appears from the beginning very different than the fast cooldown. Figure 6.23 (a) show the moment before the superconducting transition really starts. It is possible to notice how the temperature is now rather uniform around the cavity. In Figure $6.23(\mathrm{~b})$, is shown the beginning of the transition which starts from the top of the cavity equator. The warmest area of the cavity shows $T \sim 9.8 \mathrm{~K}$, indicating 


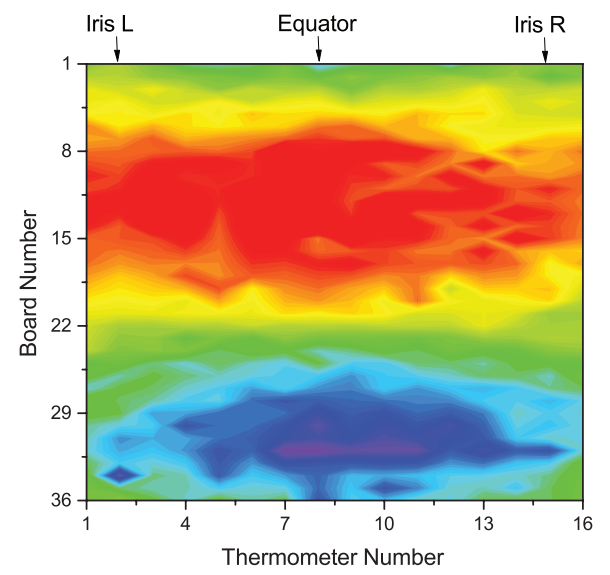

(a)

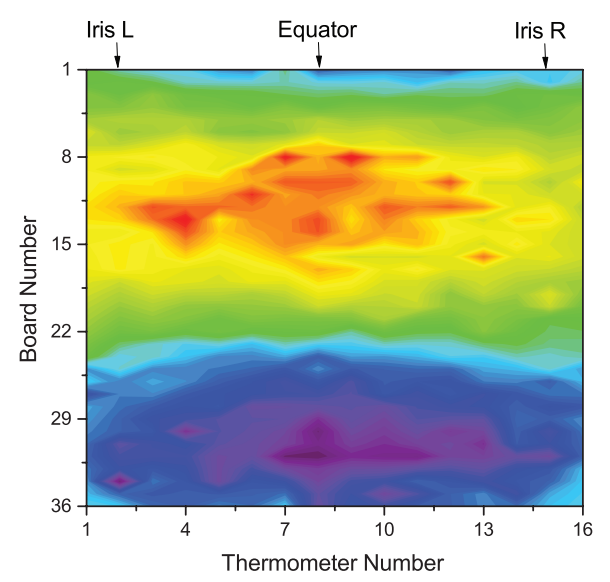

(c)

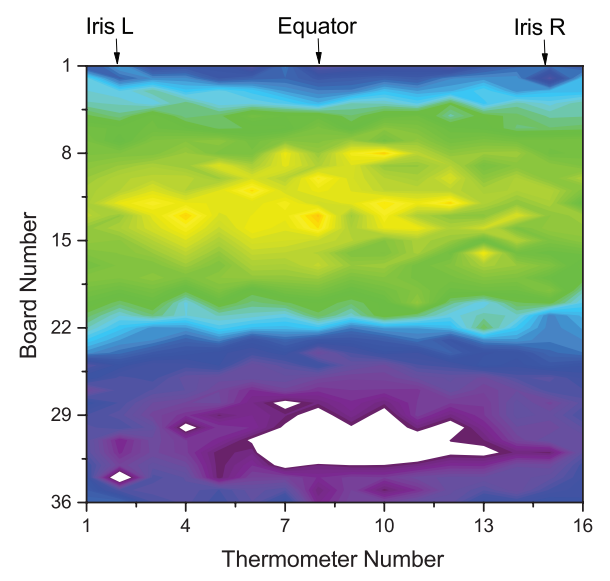

(e)
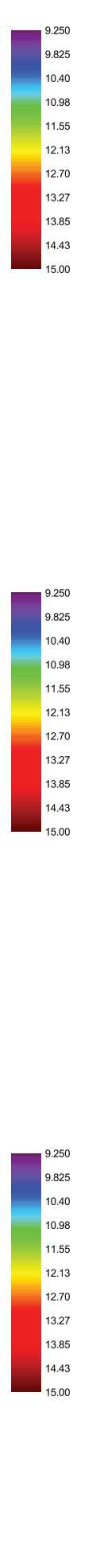

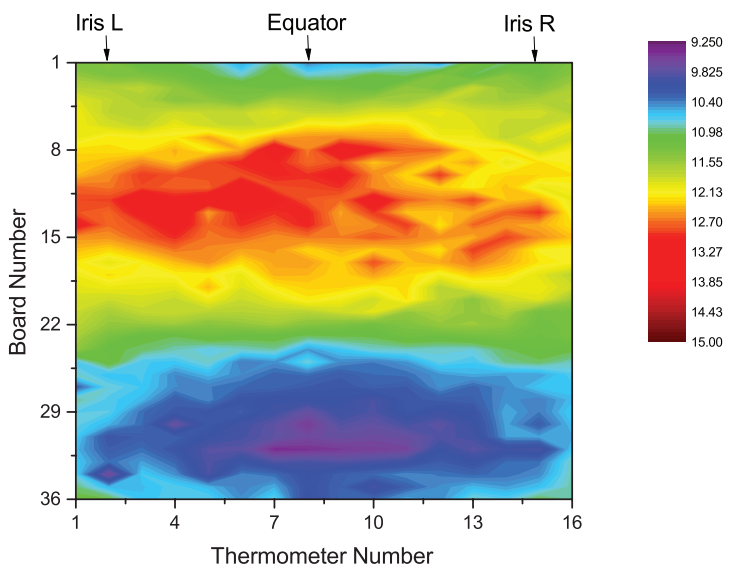

(b)

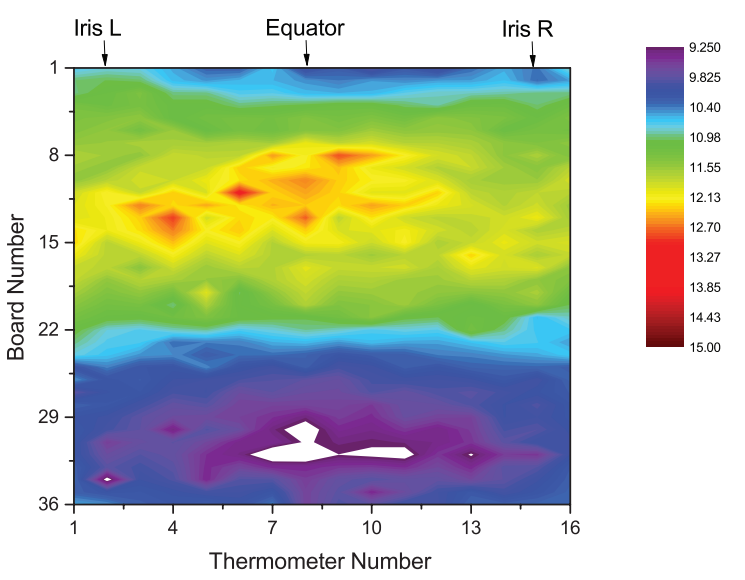

(d)

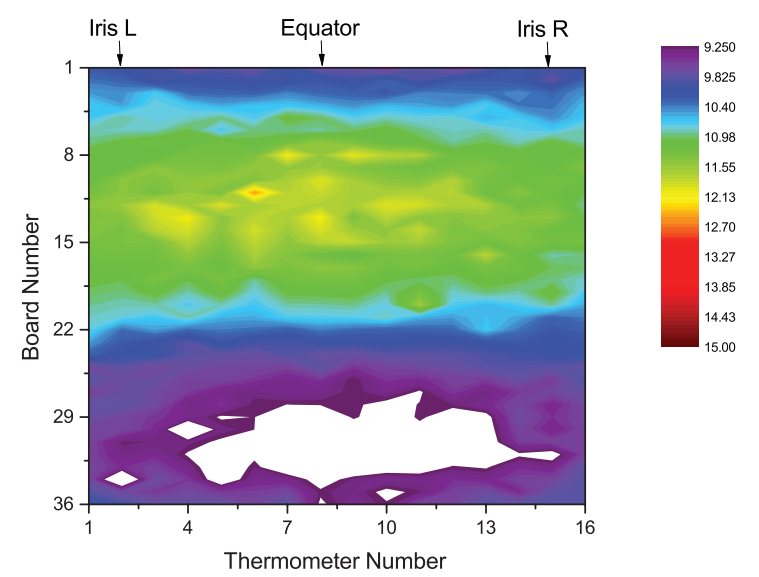

(f)

Figure 6.20. T-map images recorded during a fast cooldown. 


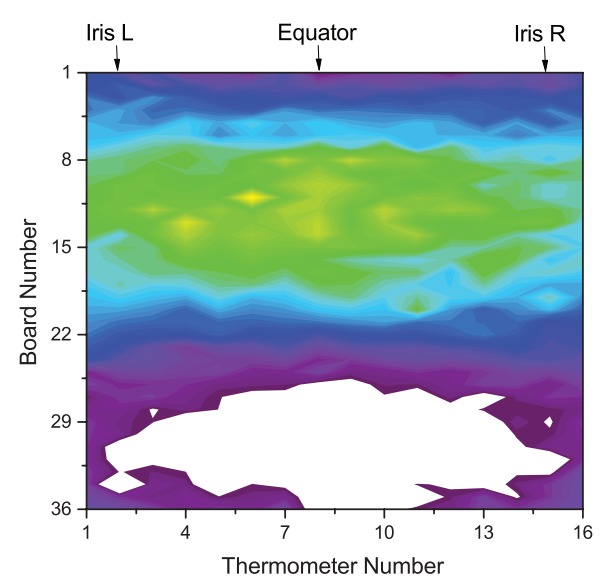

(a)

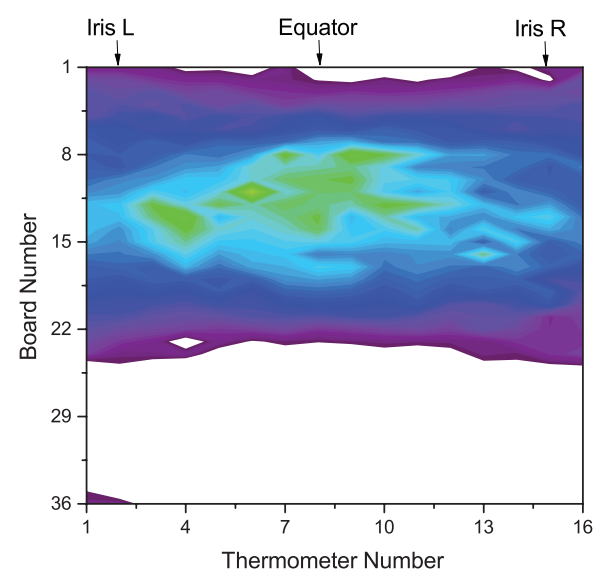

(c)

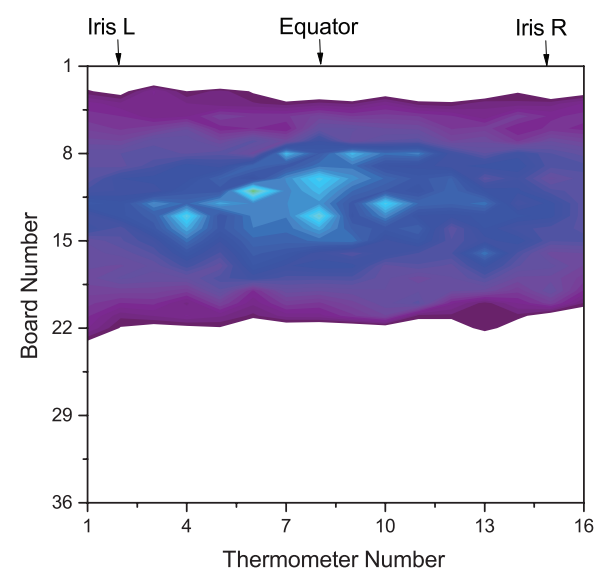

(e)
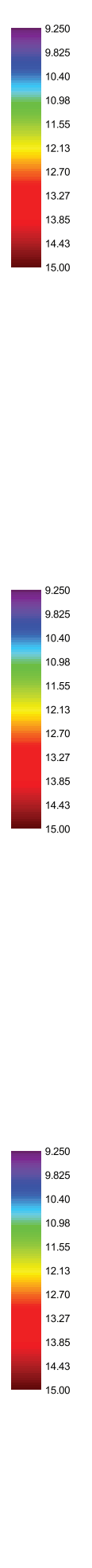
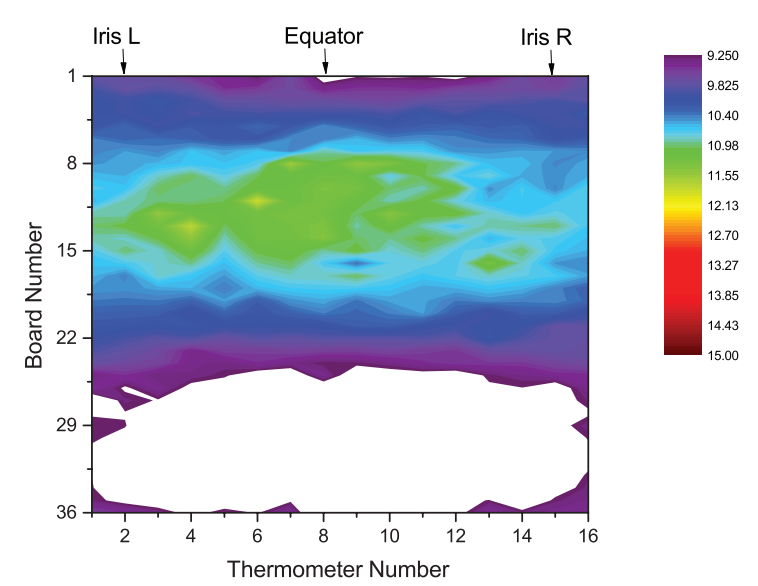

(b)

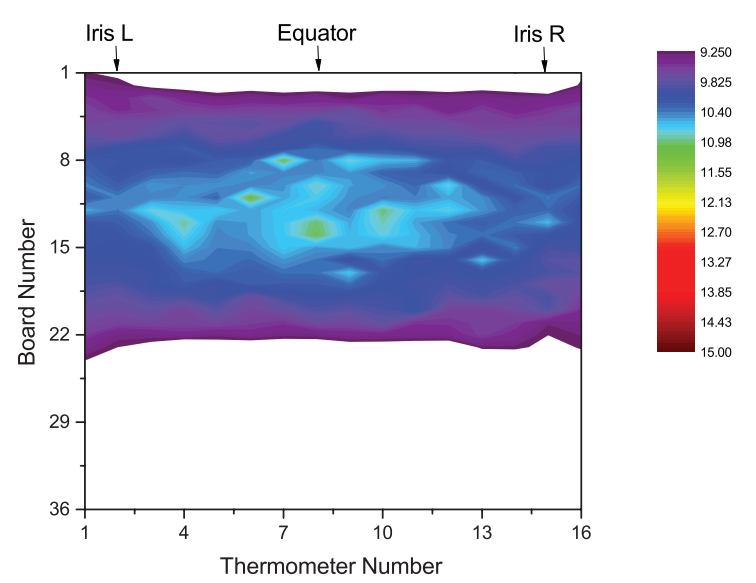

(d)

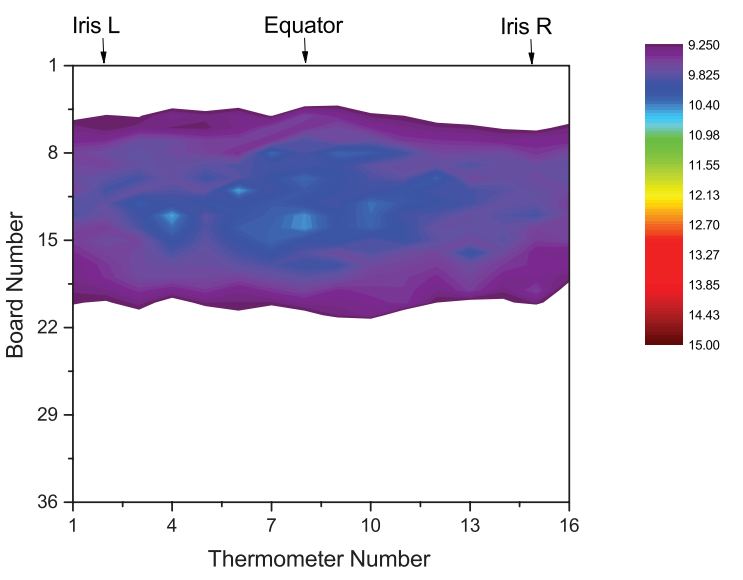

(f)

Figure 6.21. T-map images recorded during a fast cooldown. 


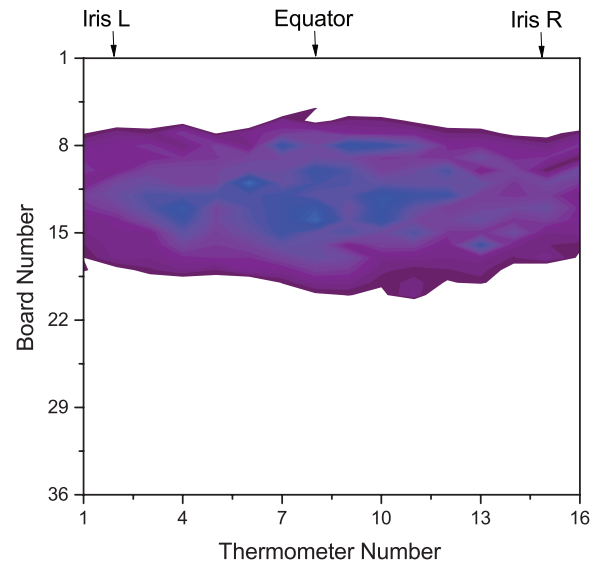

(a)

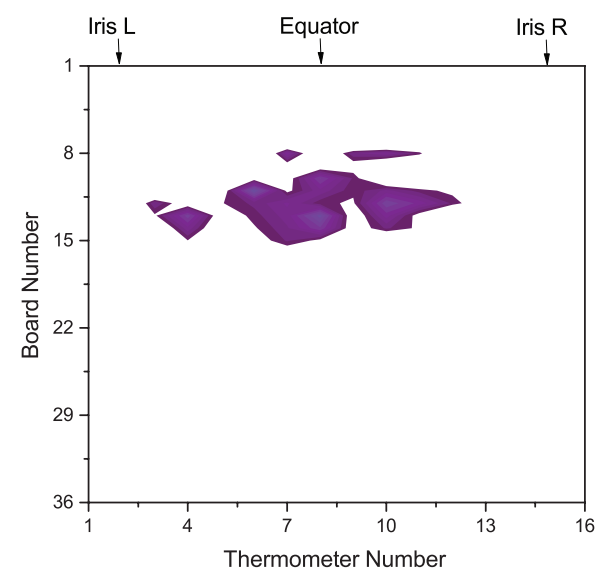

(c)

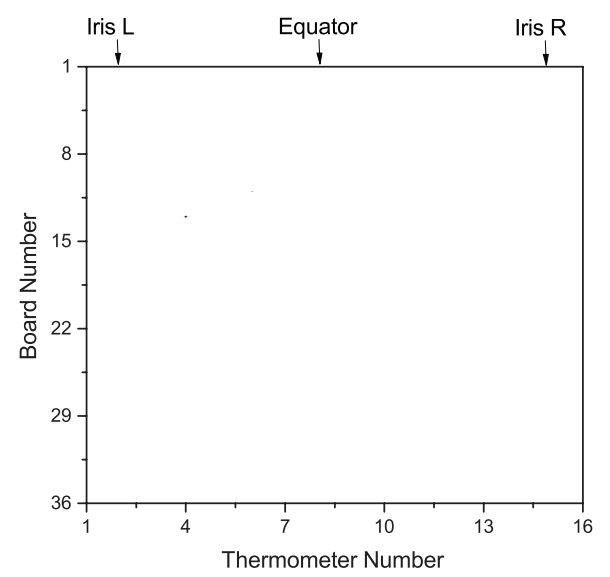

(e)
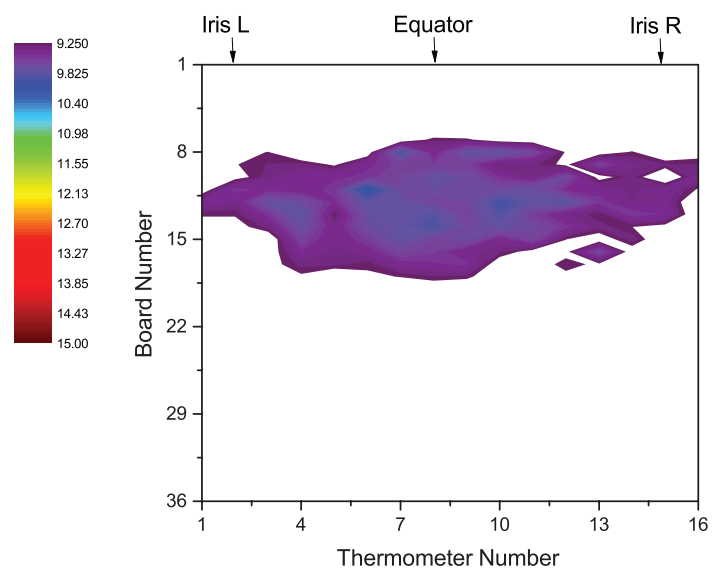

(b)
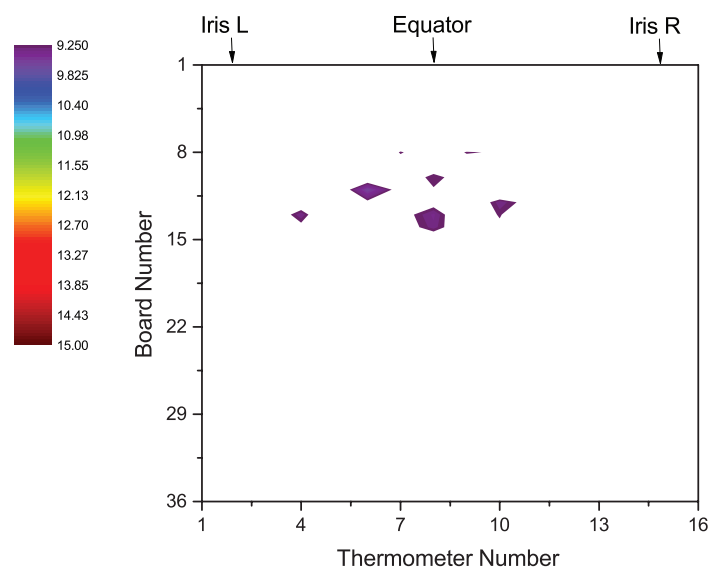

(d)
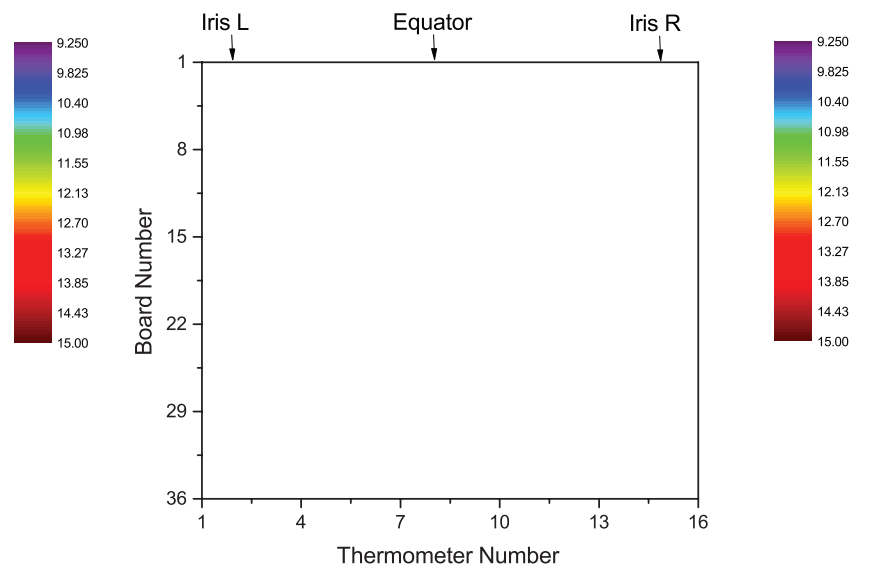

(f)

Figure 6.22. T-map images recorded during a fast cooldown. 
that there are no important thermal gradient along the cavity during this type of cooldown. Moreover these warmer zones are randomly distributes around the cavity. The same is true for the cold zones, indeed from Figures 6.23 (c)-(f) and Figures 6.24 (a)-(f) it is possible to notice how the SC regions nucleate in random spot of the cavity. The further images show the growth of the superconducting areas and also nucleation of new SC regions. Toward the end of the cavity transition, Figures 6.26 (d)-(f) and Figures 6.27 (a)-(f) show the presence of several normal-conducting regions encircle by superconducting material.

These macroscopic islands of NC phase might be responsible of the complete magnetic flux trapping observed during the slow cooldown. The magnetic field concentrates along these $\mathrm{NC}$ regions during the cavity transition and then, even once the superconducting transition is complete, it is not allowed to escape anymore from the material. Indeed, being surrounded by SC phases, the field does not have any energetically favorable path to follow to be expelled from the superconductor.

Since these NC regions are randomly distributed all over the surface, the magnetic field is expected to be trapped rather uniformly on the cavity surface during the slow cooldown.

In order to see the distribution of the magnetic field trapped in the cavity, the T-map is used to record the temperature of the cavity during the RF measurement. Such acquisition is done after that the magnetic field trapped in the cavity was minimized (after fast cooldown using the Helmontz coil to compensate for the external magnetic field), and after that the cavity trapped $10 \mathrm{mG}$ during a slow cooldown. The images acquired at $1.5 \mathrm{~K}$ and $27.5 \mathrm{MV} / \mathrm{m}$ are shown in Figure 6.29. In order to better seen the difference between these two scenarios, the measurements are done after a vertical cooldown of the cavity. After the horizontal cooldown indeed the measurements are affected by the concentration of the field on top of the cavity as 


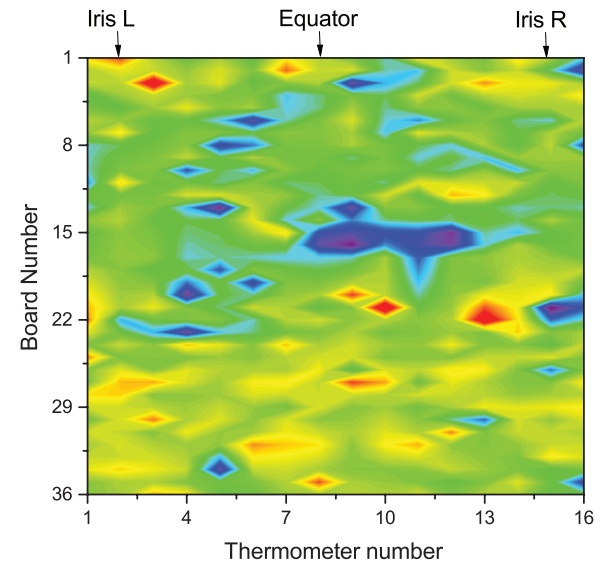

(a)

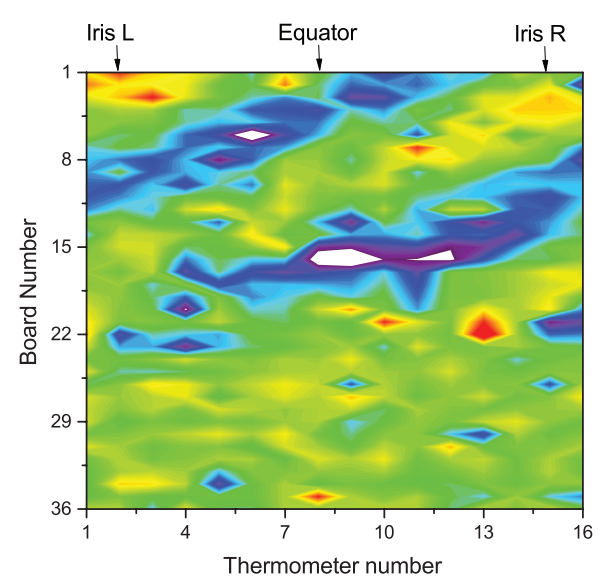

(c)

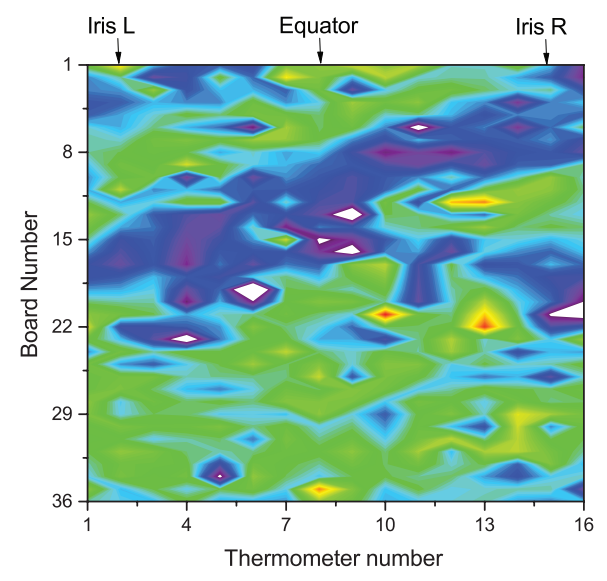

(e)
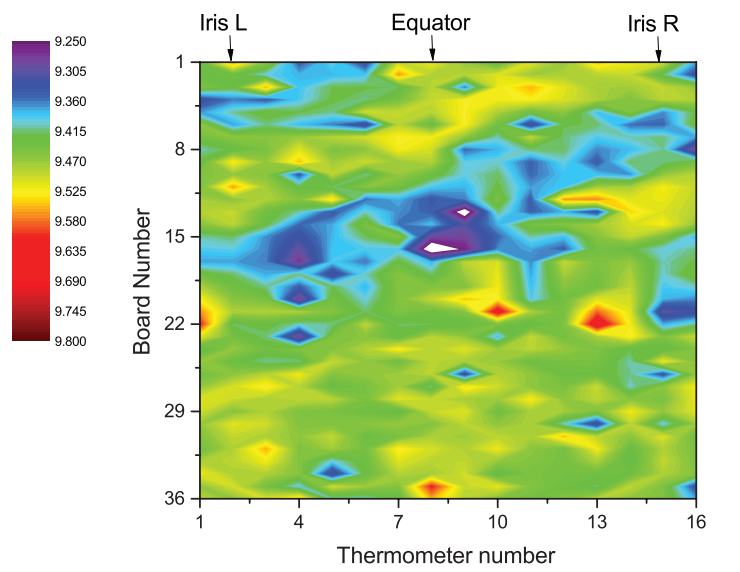

(b)
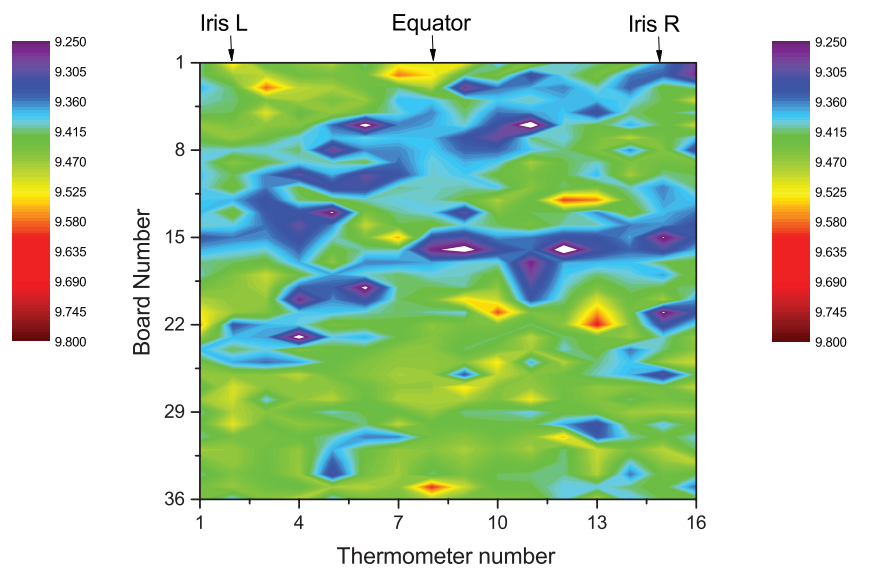

(d)
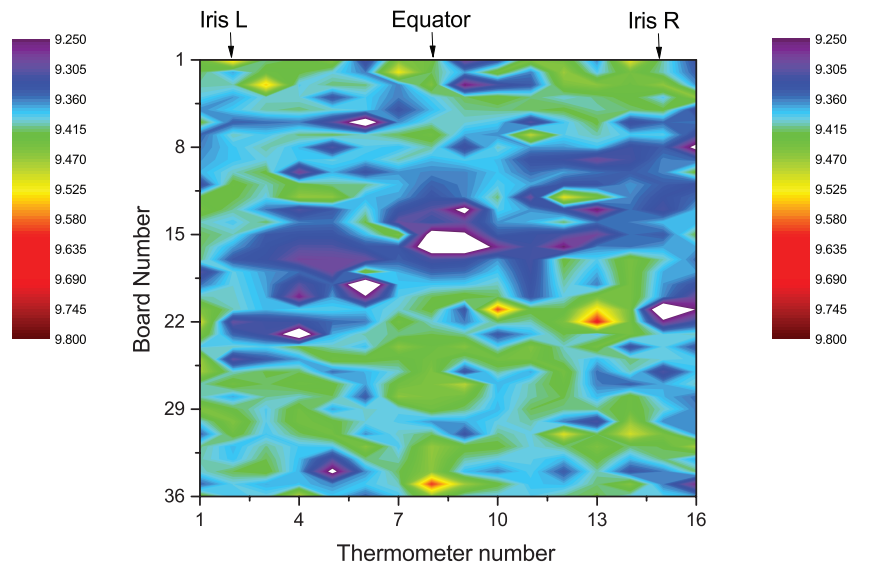

(f)

Figure 6.23. T-map images recorded during a slow cooldown. 


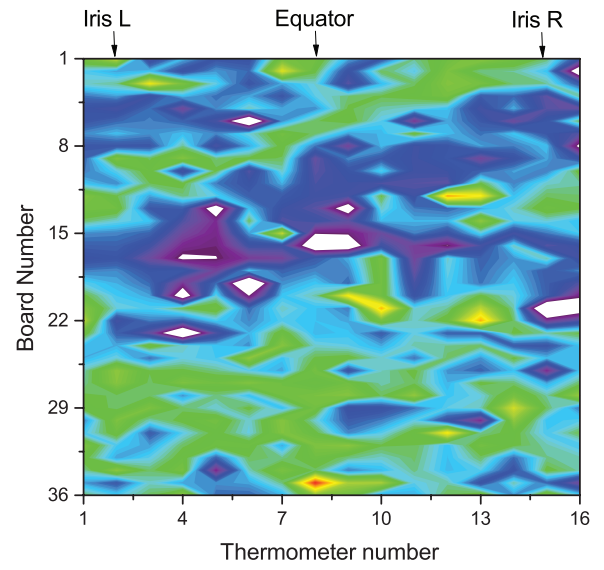

(a)

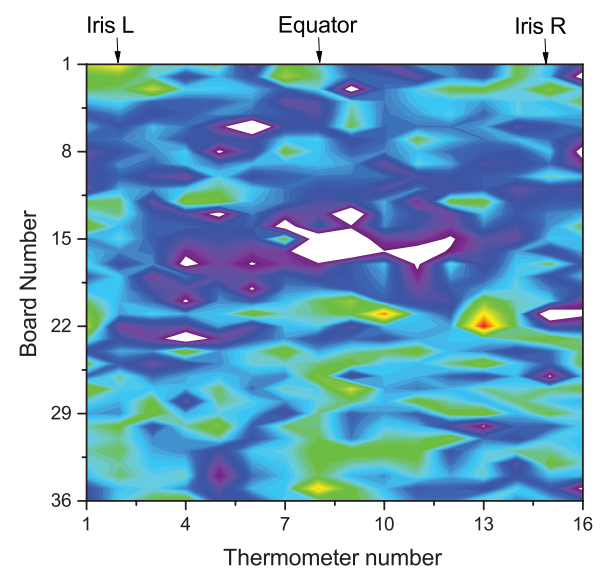

(c)

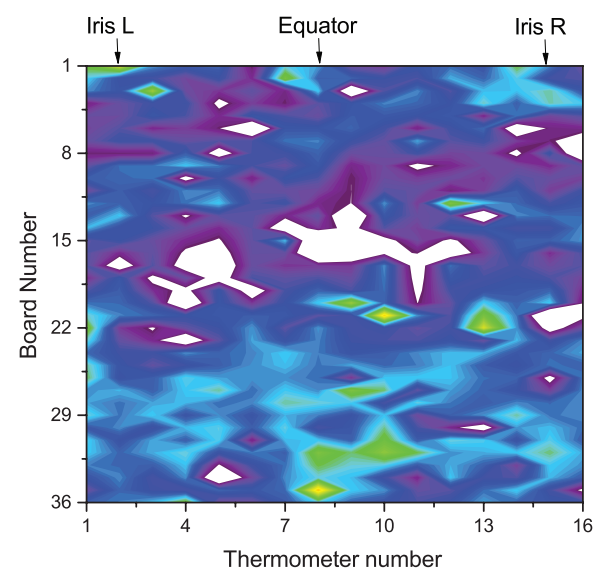

(e)
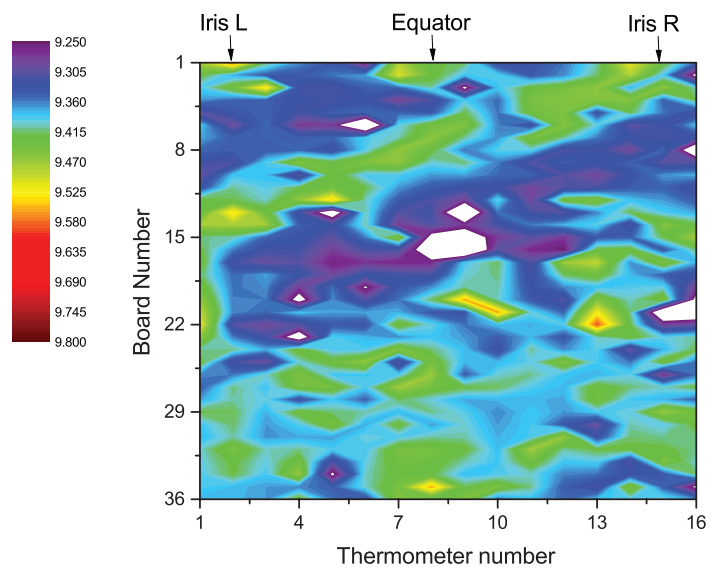

(b)
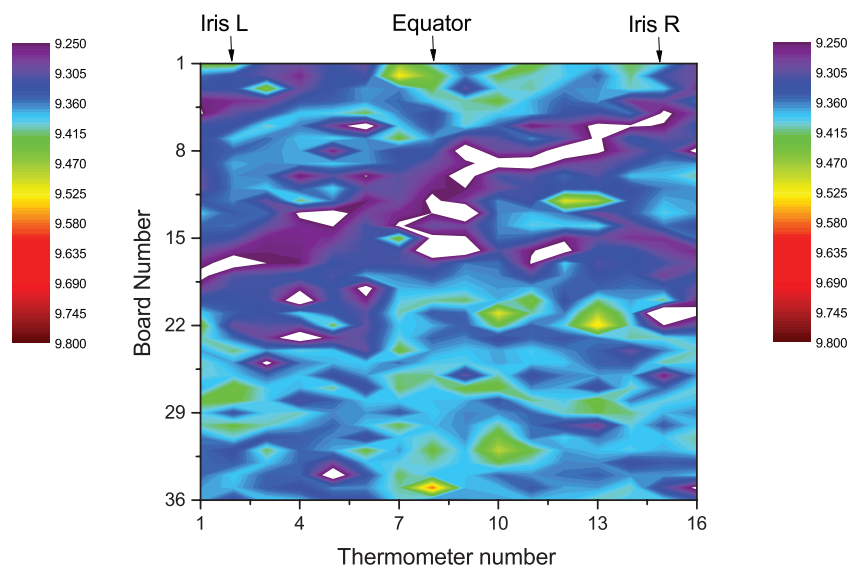

(d)
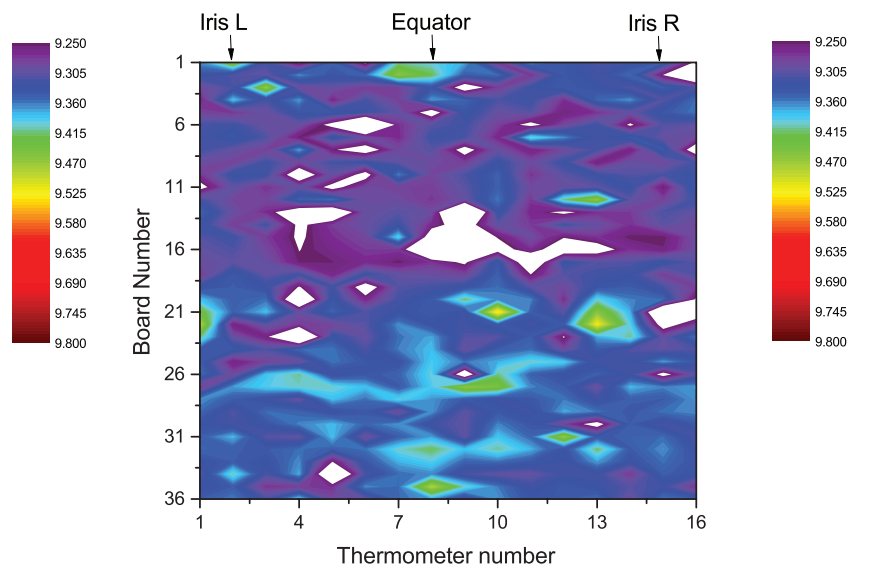

(f)

Figure 6.24. T-map images recorded during a slow cooldown. 


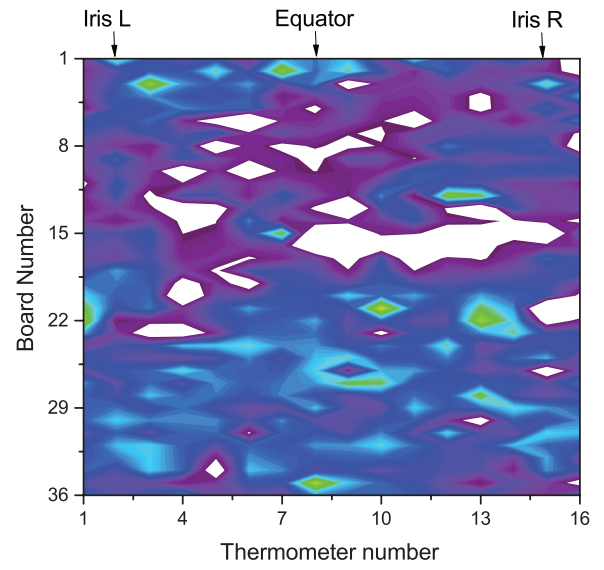

(a)

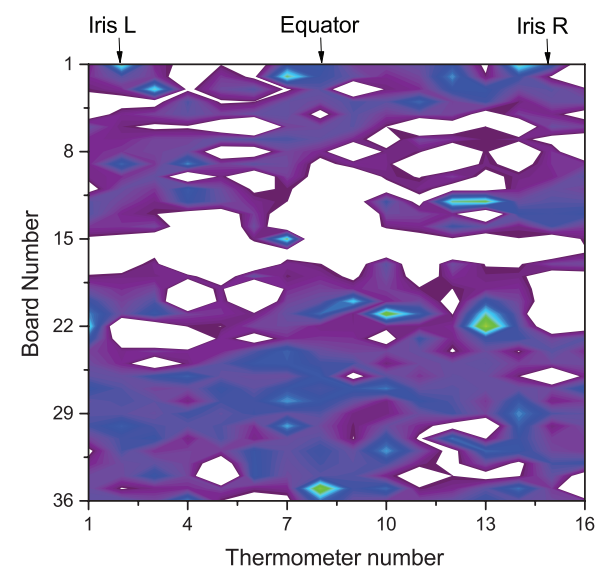

(c)

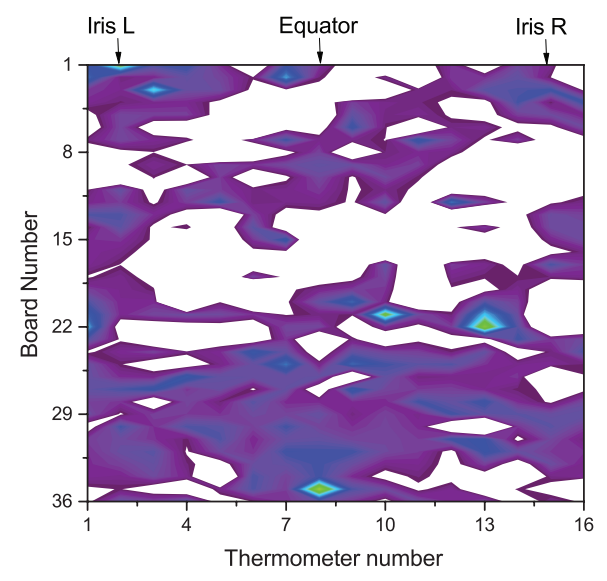

(e)
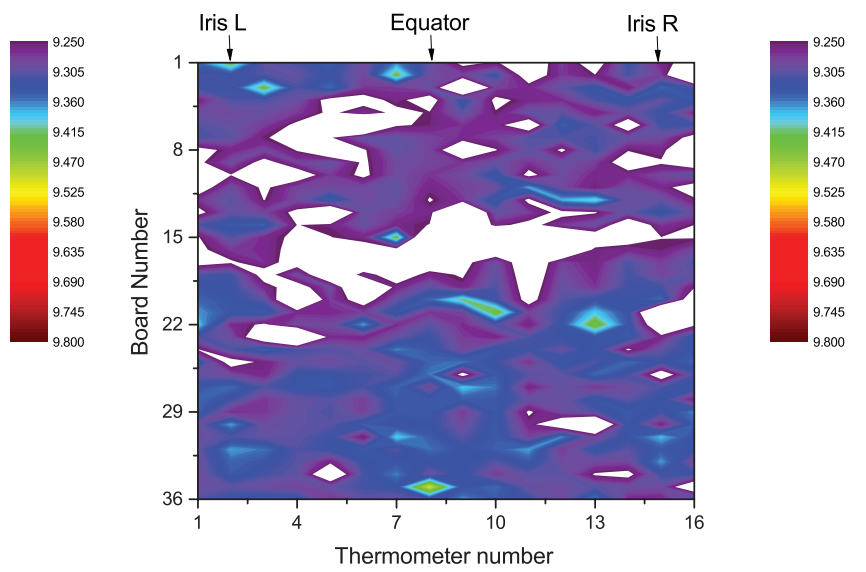

(b)

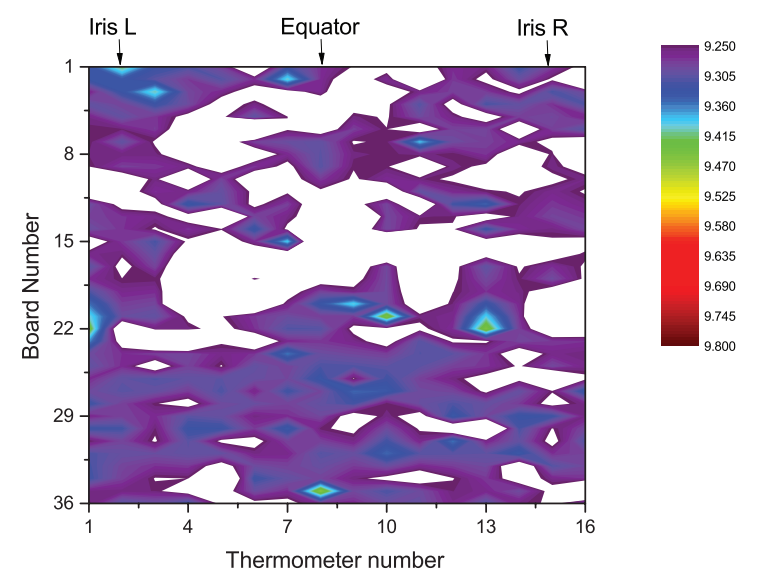

(d)
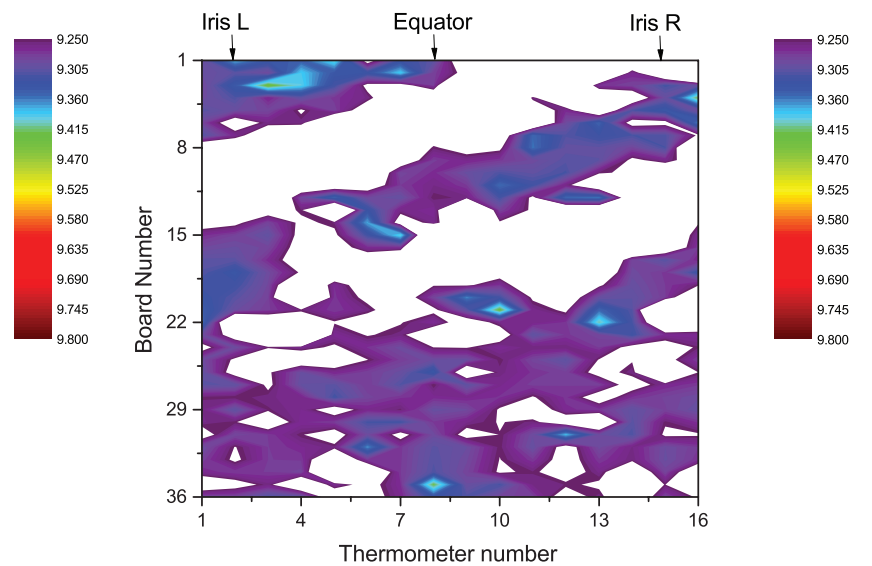

(f)

Figure 6.25. T-map images recorded during a slow cooldown. 


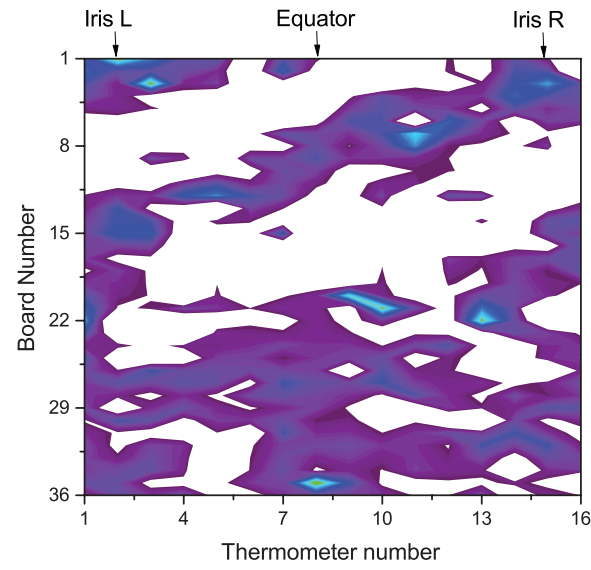

(a)

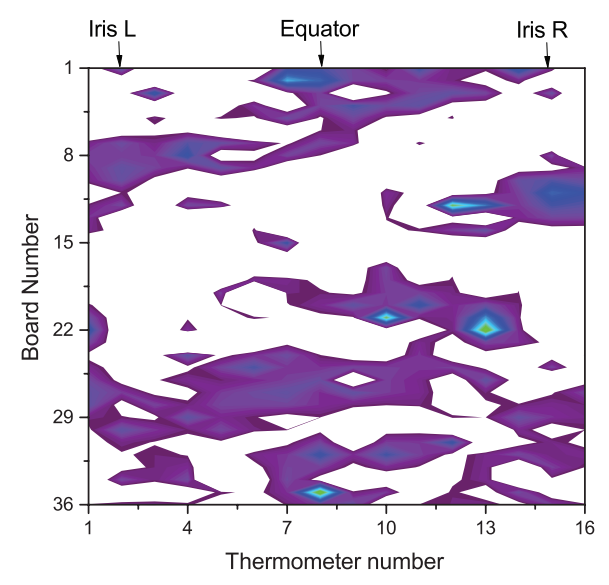

(c)

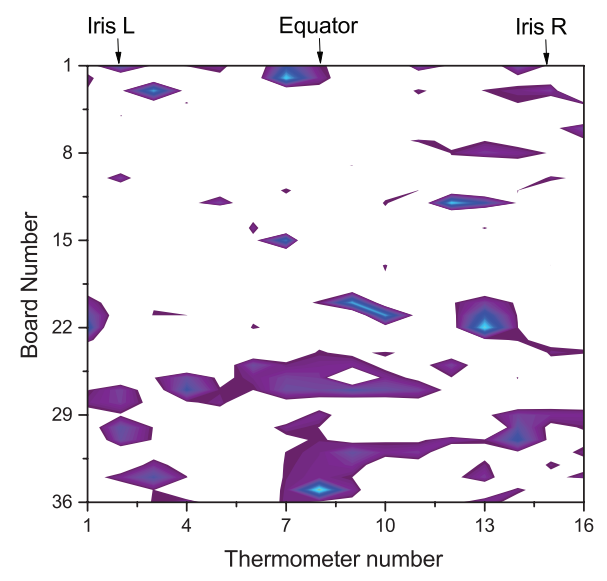

(e)
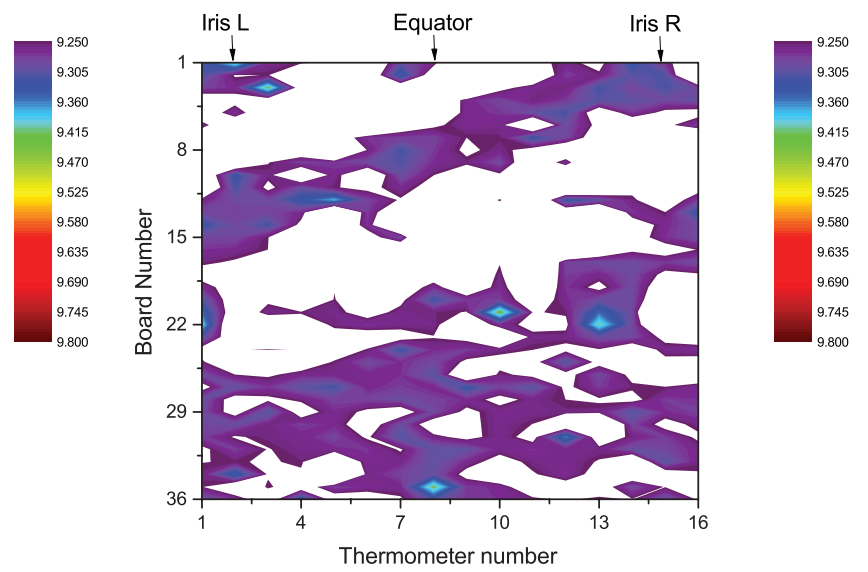

(b)
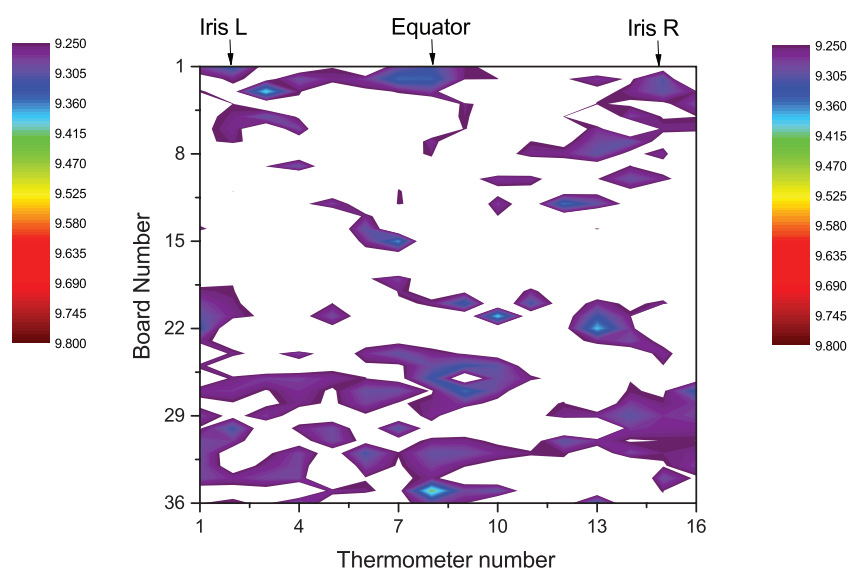

(d)
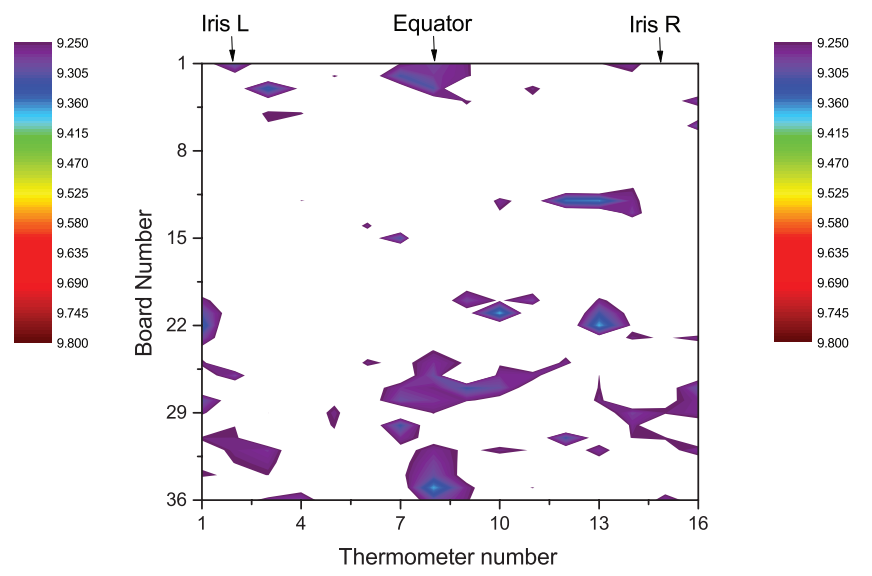

(f)

Figure 6.26. T-map images recorded during a slow cooldown. 


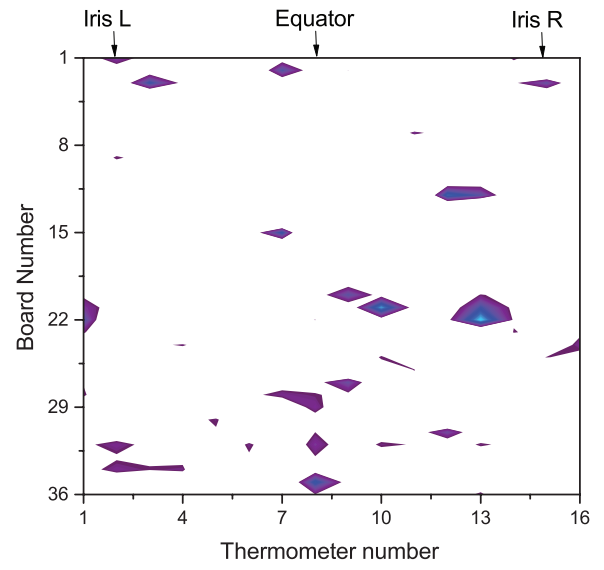

(a)

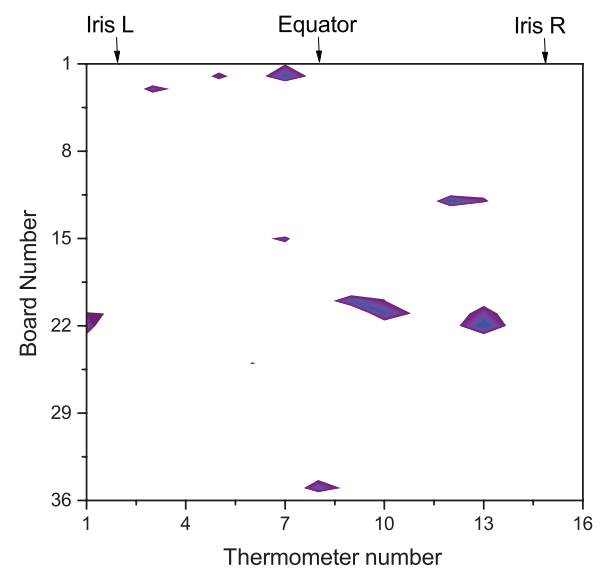

(c)

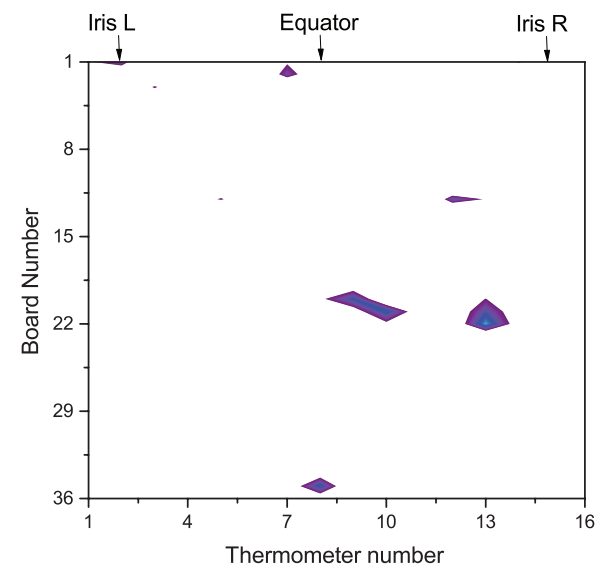

(e)
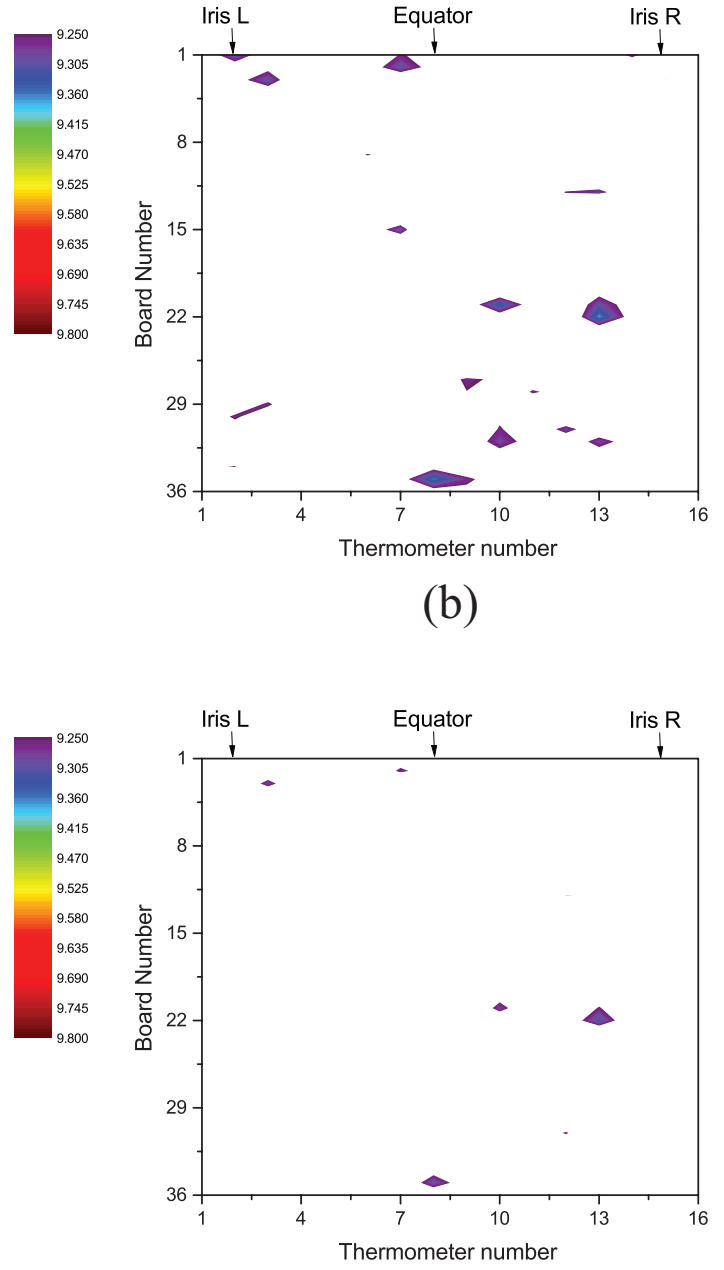

(d)
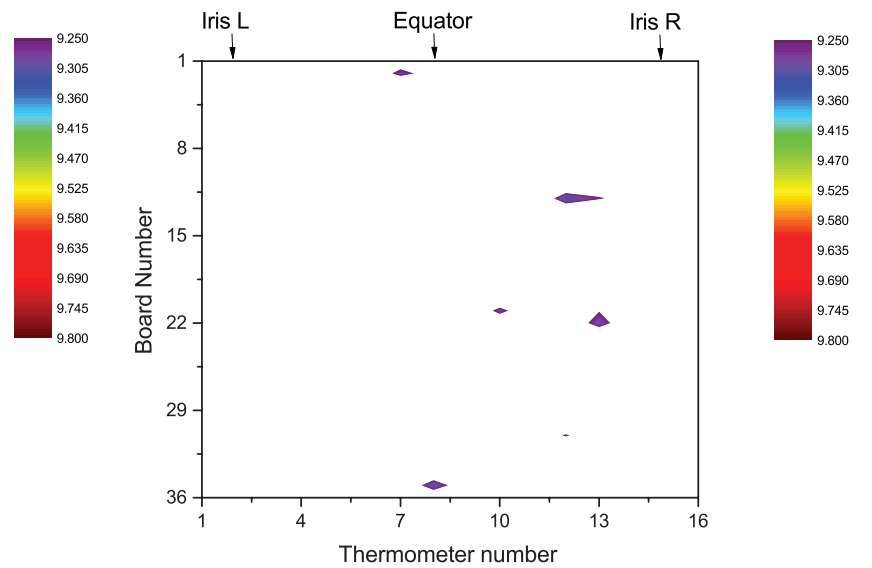

(f)

Figure 6.27. T-map images recorded during a slow cooldown. 


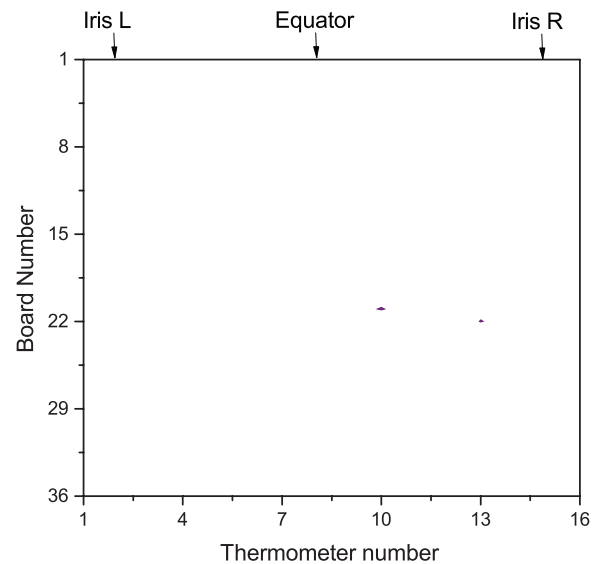

(a)

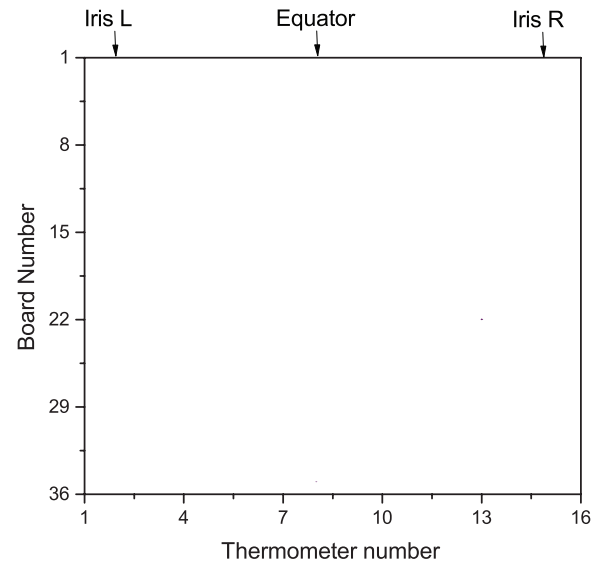

(c)
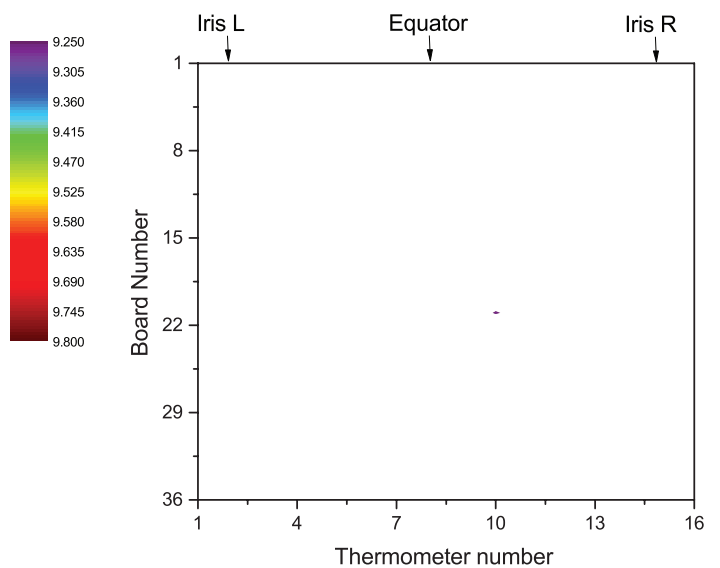

(b)
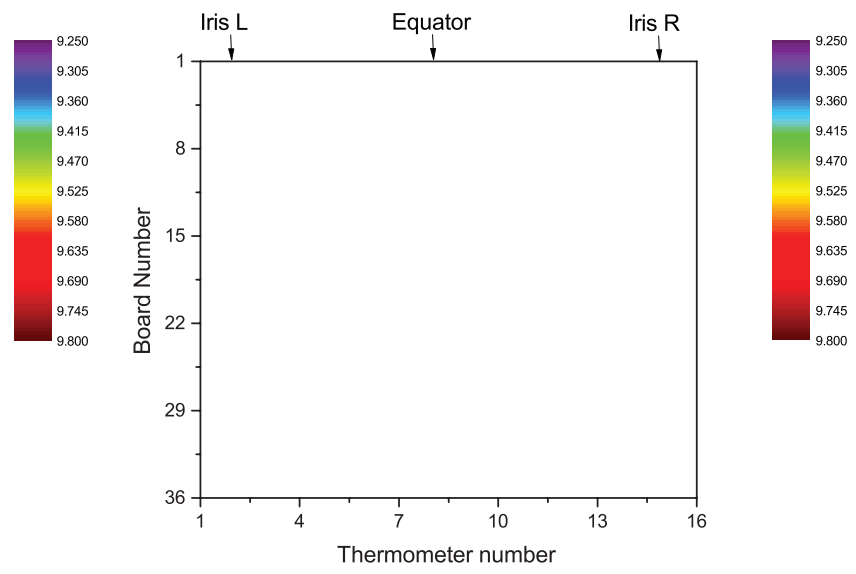

(d)

Figure 6.28. T-map images recorded during a slow cooldown. 


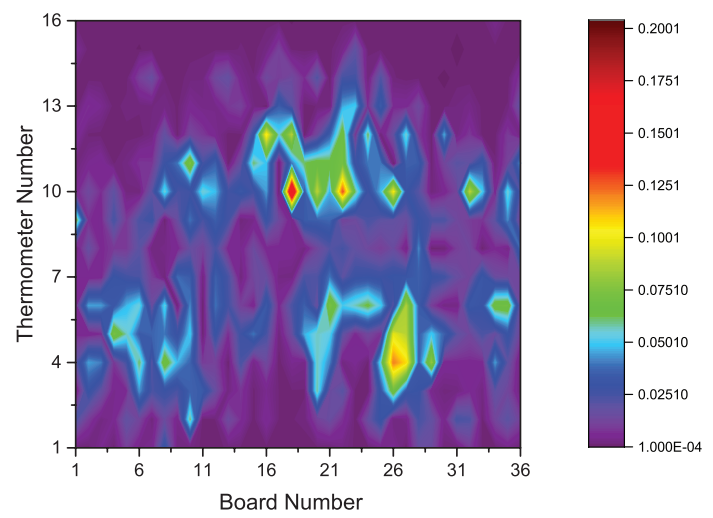

(a)

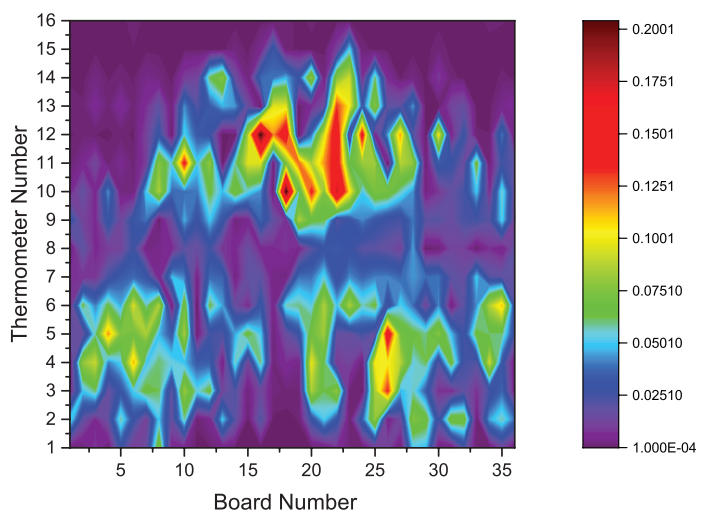

(b)

Figure 6.29. Comparison between the T-map images acquired after fast cooldown in compensated field (no magnetic field trapped) and slow cooldown in $10 \mathrm{mG}$ (10 $\mathrm{mG}$ of magnetic field trapped). Both images are acquired on the cavity AES019 at about $27 \mathrm{MV} / \mathrm{m}$ and $1.5 \mathrm{~K}$.

discussed in Chapter 5. From Figure 6.29 it is possible to see that after the slow cooldown the cavity shows larger losses, distributed all around the cavity. These losses are due to the magnetic field uniformly trapped around the cavity. Such losses are of course confirmed also by the lowered Q-factor measured after this cooldown (see Figure 6.30).

\subsection{Conclusions}

In this chapter the physics behind the magnetic flux expulsion was studied. A simple model based on the thermodynamic force of the vortex was discussed as possible mechanism capable of promoting magnetic flux expulsion. It was shown that vortices tend to move from Meissner state to mixed state regions in order to minimize their Gibbs free energy. This traduces in a movement of the vortices from the bottom to the top of the cavity during the superconducting transition.

Taking into account that vortices may also be pinned in the material, the thermodynamic force has to be greater than the pinning force in order to flushed out also pinned vortices. Including a Gaussian probability density function that 


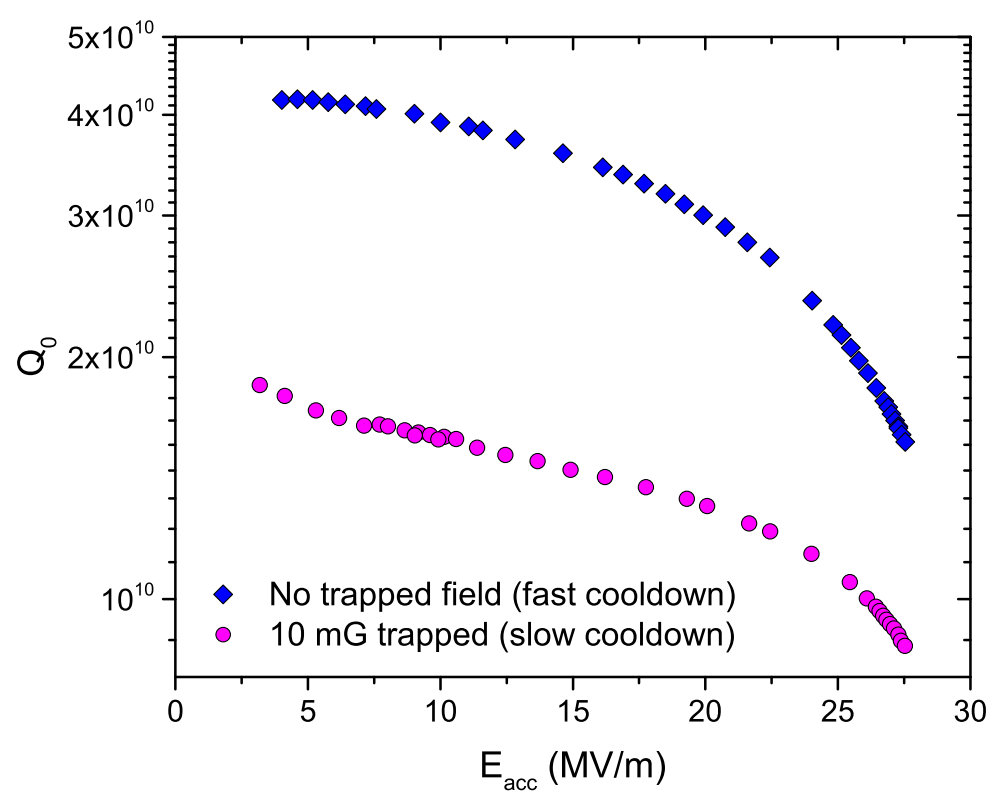

Figure 6.30. Q-factor versus accelerating field curves acquired at $1.5 \mathrm{~K}$ for the cavity AES019 acquired after the cooldown with no field trapped (blue curve) and after that the cavity trapped about $10 \mathrm{mG}$ of magnetic field (pink curve). The T-map images of Figure 6.29 are acquired during the same measurements.

takes into account the probability of expelling vortices and that is representative of the distribution of the defects in the material, the magnetic flux expulsion data of cavities with have been successfully described with the model. Most of the cavity analyzed are described by a double distribution of defects, which sometimes traduces in poor flux expulsion behavior. Indeed, in order to achieve complete flux expulsion, it is necessary to reach the maximum critical thermal gradient of the second peak of the probability density function. This may indeed explain why it is very rare to measure flux expulsion ratios equal to the ideal value of 1.74 .

Using a T-map system the dynamic of both slow and fast cooldown were recorder during the superconducting transition. It was shown that during a fast cooldown the SC transition moves sharply from the bottom to the top of the cavity. This sharp interface promotes the vortex movement due to thermodynamic force, from the bottom to the top of the cavity. 
As discussed in Chapter 5, when the cavity is horizontally cooled and the thermal gradient are large enough, this vortex movement results in magnetic flux expulsion for the magnetic component parallel to the cavity axis and flux trapping at the top of the cavity equator for the perpendicular magnetic field component. When the cavity is vertically cooled instead this situation results in magnetic flux expulsion in both cases.

The T-map images also show that during the slow cooldown, at the end of the cavity SC transition, several normal-conducting islands surrounded by the superconducting phase appear around the cavity. Therefore vortices may be concentrated in these regions. Once also these areas become superconducting, vortices can not escape, since surrounded by superconducting material. This may explain why during the slow cooldown all the magnetic field is trapped in the cavity. 


\section{CHAPTER 7 \\ SURFACE RESISTANCE DEPENDENCE ON THE MEAN FREE PATH}

Previous work has demonstrated that the radio frequency surface resistance of niobium resonators is dramatically reduced when nitrogen impurities are dissolved as interstitial in the material [19]. This effect is attributed to the lowering of the MattisBardeen surface resistance with increasing accelerating field, however the microscopic origin of this phenomenon is poorly understood. Meanwhile, an enhancement of the sensitivity to trapped magnetic field is typically observed for such cavities $[21,22,8$, $23]$.

In this chapter a systematic study on these different components contributing to the total surface resistance as a function of different levels of dissolved nitrogen, in comparison with standard surface treatments for niobium resonators is conducted. At the end of the chapter these results are added together in order to define which is the optimum surface treatment that maximizes the Q-factor of superconducting niobium resonators as a function of reasonable values of trapped magnetic field in the cavity walls. These results also provide insights on the physics behind the change in the field dependence of the Mattis-Bardeen surface resistance, and of the trapped magnetic vortex induced losses in superconducting niobium resonators.

\subsection{Introduction}

Nitrogen-doping is a surface treatment which allows nitrogen atoms to be absorbed as interstitial impurities in the niobium lattice. This treatment has shown a dramatic improvement of superconducting radio-frequency (SRF) properties. In particular, as shown in Chapter 4, the cavity quality factor, which is inversely proportional to the power dissipated on the cavity walls, can increase by a factor of three at medium values of accelerating field $\left(E_{\text {acc }}=16 M V / m\right)[19]$. 
The RF surface resistance $R_{s}$ is the material parameter that determines the Q-factor values: $Q_{0}=G / R_{\mathrm{s}}$, where $G=270 \Omega[3]$ is the geometrical factor which is independent on material properties. It is important to take into account that, as explained in Chapter 3, the RF surface resistance can be decomposed in two contributions, one temperature dependent called BCS surface resistance $\left(R_{\mathrm{BCS}}\right)$, and one temperature independent called residual resistance $\left(R_{\text {res }}\right)$.

In this chapter these two surface resistance contributions are analyzed for bulk niobium resonators, looking at both the mean free path and the RF field dependencies. The findings here reported allow a much better understanding of which surface treatment is required to maximize the Q-factor for a certain RF field, taking into account the external DC magnetic field trapped during the cooldown through the superconducting (SC) transition.

Q-factor maximization is extremely beneficial in order to decrease the cryogenic cost of continuous-wave accelerators. For this reason the Linear Coherent Light Source (LCLS-II) at SLAC embraced the nitrogen-doping technology as treatment for the SRF cavities of the superconducting upgrading of their linear accelerator [20].

As seen in Chapter 3, the BCS surface resistance was defined by Mattis and Bardeen [40]. Based on the Bardeen-Cooper-Schrieffer theory of superconductivity [37], $R_{\mathrm{BCS}}$ decays exponentially with the temperature and depends on several material parameters, such as: London penetration depth $\lambda_{\mathrm{L}}$, coherence length $\xi_{0}$, energy gap $\Delta$, critical temperature $T_{\mathrm{c}}$ and mean free path $\ell$. Of most interest here is that from the Mattis-Bardeen calculation, $R_{\mathrm{BCS}}$ as a function of the mean free path shows a minimum around $\xi_{0} / 2[64]$.

It is well known that nitrogen-doping affects the BCS contribution [19] which, in contrary of what happens with standard treatments, decreases with the accelerating 
field. This results in an increasing of Q-factor with accelerating field called anti-Qslope. The mechanisms that govern the anti-Q-slope are not well understood yet, even though some theories have been proposed $[65,66]$.

This study adds important insight on the BCS surface resistance field dependence, suggesting that the decreasing of $R_{\mathrm{BCS}}$ may be due to an increasing of the energy gap $\Delta$ with the RF field.

The introduction of interstitial impurities and the subsequent change in mean free path affects also $R_{\text {res }}$. Principal sources of residual losses are: condensed gasses, material inclusions, hydrides and trapped magnetic flux [25]. This last contribution defines the trapped flux sensitivity which in turns depends strongly on the mean free path [21]. Here a complete and detailed study which gives a clear picture of trapped flux dissipation in SRF niobium cavities is shown, from its dependence on the mean free path to its dependence on the RF field. This part of the study is of crucial importance in order to understand both the surface treatment of SRF cavities and the level of magnetic field shielding needed in cryomodules.

As seen in Chapters 5 and 6, this additional residual resistance contribution is due to ambient magnetic field that may be trapped during the SC transition. The amount of trapped flux depends on both the amount of external magnetic field which surrounds the cavity during the SC transition, and on the cooldown details, which affects the magnetic flux trapping efficiency. In particular, fast cooldowns with large thermal gradients along the cavity length help to obtain efficient magnetic flux expulsion, while slow and homogeneous cooling through transition leads to full flux trapping $[54,67,68,6]$.

\subsection{Experimental procedure}

The cavities analyzed are single cell 1.3 GHz TESLA-type bulk niobium cav- 


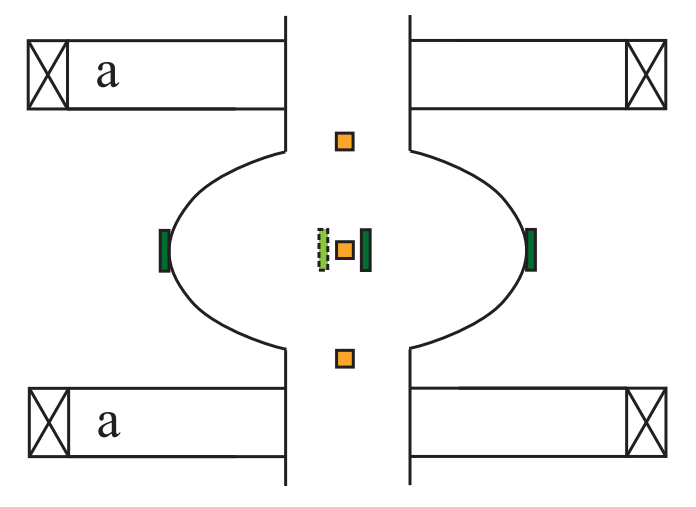

Figure 7.1. Scheme of the cavity instrumentation needed to monitor the external magnetic field and the cavity temperature during the cooldowns. The thermometers are represented as orange squares, while the green rectangles represent the fluxgates. The Helmholtz coil is indicated in the figure with the letter a.

ities [3], processed with different surface treatment: N-doping, EP, BCP, $120{ }^{\circ} \mathrm{C}$ baking (see Chapter 4 for more detail of these treatments). The surface treatment of the analyzed cavities are summarized in Table 7.1 .

The values of mean free path were estimated by interpolating with SRIMP [2] the variation of the penetration depth as a function of the temperature during the cavities warm up, unless otherwise specified in Table 7.1. The mean free path uncertainty reported is the one directly estimated by the interpolation with SRIMP.

The uncertainties on the sensitivity and BCS surface resistance values are extrapolated via the uncertainty propagation method, setting a priory errors for the magnetic field (10\%) and Q-factors (depending on the coupling parameter during the RF test, as explained in Reference [44]) measurements. A schematic of the instrumentation used to characterize the trapped flux surface resistance is shown in Figure 7.1. Helmholtz coils adjust the magnetic field around the cavity, three Bartington single axis fluxgate magnetometers monitor the external magnetic field at the cavity equator and thermometers monitor the cooldown details.

In order to estimate the trapped flux surface resistance, each cavity is measured 
Table 7.1. Summary of the cavity surface treatments with the estimated values of mean free path, trapped flux sensitivity at 5 $\mathrm{MV} / \mathrm{m}$ and $2 \mathrm{~K}$ BCS surface resistance at $16 \mathrm{MV} / \mathrm{m}$ (unless specified otherwise).

\begin{tabular}{|c|c|c|c|c|}
\hline Cavity Name & Surface Treatment & $\ell(\mathrm{nm})$ & Sensitivity $(\mathrm{n} \Omega / \mathrm{mG})$ & $R_{\mathrm{BCS}}(n \Omega)$ \\
\hline AES014_2 & Additional $120{ }^{\circ} \mathrm{C}$ bake & $16 \pm 1.6^{a}$ & $0.39 \pm 0.05$ & $12 \pm 2$ \\
\hline $\mathrm{ACC} 005$ & $120^{\circ} \mathrm{C}$ bake & $16 \pm 1.6^{a}$ & - & $10.2 \pm 0.7$ \\
\hline AES021 & Air $30 \min \left(\right.$ at $\left.800{ }^{\circ} \mathrm{C}\right)+3 \mu \mathrm{m} \mathrm{EP}$ & $30 \pm 20$ & $0.58 \pm 0.08$ & $4.6 \pm 0.8^{b}$ \\
\hline AES005 & 1 hour $N_{2}\left(\right.$ at $\left.1000^{\circ} \mathrm{C}\right)+60 \mu \mathrm{m} \mathrm{EP}$ & $40 \pm 4^{c}$ & - & $4.5 \pm 0.7$ \\
\hline AES021_2 & Additional $5 \mu \mathrm{m} \mathrm{EP}$ & $57 \pm 5$ & $1.1 \pm 0.16$ & $4.5 \pm 1.8$ \\
\hline ACC002 & $20 \min N_{2}\left(\right.$ at $\left.800^{\circ} \mathrm{C}\right)+5 \mu \mathrm{m} \mathrm{EP}$ & $70 \pm 10$ & $1.6 \pm 0.2$ & $4.3 \pm 0.8$ \\
\hline CBMM & $2 / 6 \min N_{2}\left(\right.$ at $\left.800{ }^{\circ} \mathrm{C}\right)+5 \mu \mathrm{m} \mathrm{EP}$ & $90 \pm 10$ & $1.52 \pm 0.2$ & $4.9 \pm 0.7$ \\
\hline PAV009 & $20 \min N_{2}\left(\right.$ at $\left.900^{\circ} \mathrm{C}\right)+5 \mu \mathrm{m} \mathrm{EP}$ & $90 \pm 10$ & $1.6 \pm 0.18$ & $3.5 \pm 0.7$ \\
\hline AES019_3 & Additional $30 \min N_{2}\left(\right.$ at $\left.800^{\circ} \mathrm{C}\right)+5 \mu \mathrm{m} \mathrm{EP}$ & $100 \pm 2$ & $1.25 \pm 0.18$ & $4 \pm 2$ \\
\hline AES011 & $2 / 6 \min N_{2}\left(\right.$ at $\left.800^{\circ} \mathrm{C}\right)+5 \mu \mathrm{m} \mathrm{EP}$ & $122 \pm 3$ & $1.2 \pm 0.14$ & $4.3 \pm 1.9$ \\
\hline AES009 & $2 / 6 \min N_{2}\left(\right.$ at $\left.800^{\circ} \mathrm{C}\right)+5 \mu \mathrm{m} \mathrm{EP}$ & $128 \pm 5$ & $1.3 \pm 0.3$ & $3.7 \pm 0.4$ \\
\hline AES017_2 & Additional $2 / 6 \min N_{2}\left(\right.$ at $\left.800{ }^{\circ} \mathrm{C}\right)+5 \mu \mathrm{m} \mathrm{EP}$ & $128 \pm 4$ & $1.2 \pm 0.15$ & $4.9 \pm 0.4$ \\
\hline AES019 & $10 \min N_{2}\left(\right.$ at $\left.800^{\circ} \mathrm{C}\right)+5 \mu \mathrm{m} \mathrm{EP}$ & $134 \pm 7$ & $1.0 \pm 0.15$ & $4.7 \pm 0.7$ \\
\hline AES017 & $2 / 6 \min N_{2}\left(\right.$ at $\left.800^{\circ} \mathrm{C}\right)+5 \mu \mathrm{m} \mathrm{EP}$ & $182 \pm 7$ & $0.8 \pm 0.11$ & $4 \pm 1$ \\
\hline AES018 & 30 min $\mathrm{He}\left(\right.$ at $\left.800^{\circ} \mathrm{C}\right)$ & $860 \pm 90$ & $0.43 \pm 0.08$ & $12 \pm 2$ \\
\hline AES014 & $120 \mu \mathrm{m} \mathrm{EP}$ & $860 \pm 90^{d}$ & - & $12.3 \pm 0.2$ \\
\hline AES019_2 & Additional $50 \mu \mathrm{m} \mathrm{BCP}$ & $1200 \pm 200$ & $0.30 \pm 0.04$ & $11 \pm 2$ \\
\hline
\end{tabular}

a Calculated with LE- $\mu \mathrm{SR}$ on a cavity cut-out representative for the $120^{\circ} \mathrm{C}$ baking treatment.

b Calculated at $5 \mathrm{MV} / \mathrm{m}$.

c Calculated with LE- $\mu$ SR on a cavity cut-out.

d Fixed at the same value of AES018. 
after two different cooldowns: i) compensating the magnetic field outside the cavity during the SC transition, ii) cooling slowly the cavity with about $10-20 \mathrm{mG}$ of external magnetic field. After each cooldown, the cavities are tested at the vertical test facility at Fermilab.

The residual resistance $R_{\text {res }}$ is here defined as sum of two terms: the trapped flux residual resistance, $R_{\mathrm{fl}}$, and the "intrinsic" residual resistance, $R_{0}$. In this way it is possible to distinguish the effect of trapped flux from other contributions, therefore:

$$
R_{\mathrm{s}}\left(T, B_{\text {trap }}\right)=R_{\mathrm{BCS}}(T)+R_{\mathrm{fl}}\left(B_{\mathrm{trap}}\right)+R_{0}
$$

where $T$ is the temperature and $B_{\text {trap }}$ the trapped field. Since at very low temperatures $R_{\mathrm{BCS}}$ becomes negligible, the Q-factor is measured at $T=1.5 \mathrm{~K}$ and the residual resistance is calculated as $R_{\mathrm{res}}=G / Q(1.5 \mathrm{~K})$. If during the cooldown the amount of trapped flux is minimized, then: $R_{\mathrm{fl}} \simeq 0$ and $R_{\mathrm{res}} \simeq R_{0}$. In order to obtain $R_{\mathrm{f}} \simeq 0$, the magnetic field outside the cavity is compensated during the cooldown through the $\mathrm{SC}$ transition. The average value of magnetic field measured at the cavity equator is always kept lower than $1 \mathrm{mG}$. Alternatively, when possible, the measurement was done after a complete magnetic flux expulsion $\left(B_{\mathrm{SC}} / B_{\mathrm{NC}} \sim 1.74\right.$ at the equator) [59]. It was observed that these two methods gave the same results within the measurements uncertainties. On the other hand, after the cavity trapped some external field: $R_{\text {res }}\left(B_{\text {trap }}\right)=R_{\mathrm{fl}}\left(B_{\text {trap }}\right)+R_{0}$.

$R_{\mathrm{BCS}}$ and $R_{\mathrm{f}}$ are therefore estimated as follows:

$$
\begin{aligned}
& R_{\mathrm{BCS}}(2 K)=R_{\mathrm{s}}(2 K)-R_{0} \\
& R_{\mathrm{fl}}\left(B_{\text {trap }}\right)=R_{\text {res }}\left(B_{\text {trap }}\right)-R_{0} .
\end{aligned}
$$

$R_{\text {res }}\left(B_{\text {trap }}\right)$ was always calculated from the RF measurements after slow cooldowns so that the amount of trapped flux tends to the amount of external field: $B_{\text {trap }} \simeq B_{\mathrm{NC}}$.

\subsection{Mean free path calculation}


The mean free path of the majority of the cavities analyzed is estimated by means of a $\mathrm{C}++$ translated version of SRIMP [2] implemented in the OriginLab data analysis program.

The cavity resonance frequency as a function of temperature during the cavity warm up is acquired in order to obtain the variation of the penetration depth, $\Delta \lambda$, as a function of $T$ close to $T_{\mathrm{c}}[64]$.

The variation of frequency during the cavity warming up is a consequence of the variation of the penetration depth with the temperature, which, for $T / T_{c}>0.8$, can be approximated as:

$$
\frac{1}{\lambda^{2}(T)}=\frac{1}{\lambda_{0}^{2}}\left[1-\left(\frac{T}{T_{c}}\right)^{4}\right] .
$$

The penetration depth $\lambda$ increases by increasing the temperature, meaning that the volume occupied by the magnetic field inside the cavity is also increasing with the temperature. This volumetric variation leads to a decreasing of the resonance frequency, in agreement with the Slater's theorem [69].

The frequency variation $\Delta f$ is indeed proportional to the variation of the penetration depth $\Delta \lambda$ :

$$
\Delta \lambda=\frac{G \Delta f}{\mu_{0} \pi f^{2}\left(T_{0}\right)},
$$

where $\Delta f=f(T)-f\left(T_{0}\right), \Delta \lambda=\lambda(T)-\lambda\left(T_{0}\right)$ and $T_{0}$ is the temperature at which the frequency is still constant, usually $T_{0} \sim 7.5 \mathrm{~K}$.

The measurements of the cavity resonance frequency were done by using a network analyzer which fed the cavity with low power.

The SRIMP code is used to interpolate $\Delta \lambda$ versus temperature. The fixed parameters of the interpolation are: critical temperature, coherence length $\left(\xi_{0}=38\right.$ $\mathrm{nm})$ and London penetration depth $\left(\lambda_{\mathrm{L}}=39 \mathrm{~nm}\right)$. The parameters obtained from 


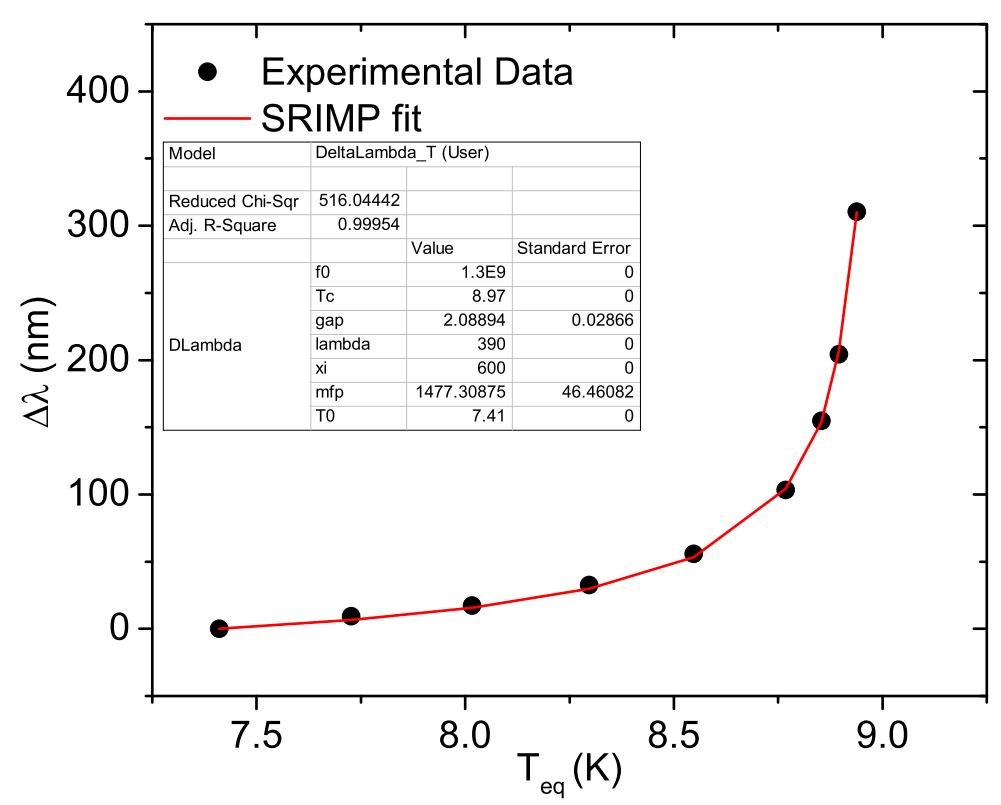

Figure 7.2. Interpolation using SRIMP of the penetration depth variation as a function of the temperature of the cavity AES011.

the interpolation are: mean free path $\ell$ and reduced energy gap $\left(\Delta / k T_{\mathrm{c}}\right)$.

An example of the fit is shown in Figure 7.2. The critical temperature is estimated from the measurement of the resonance frequency as a function of temperature. The resonance frequency drops at the normal-conducting transition, and stabilizes when the cavity is normal-conducting. The critical temperature is usually calculated as the average between the temperature of the last point of the cavity in the SC state and the first point of the cavity in the NC state.

The variation of $\Delta \lambda$ as a function of temperature differs for cavities with different mean free path values. This may be well visualize looking at the variation of $\Delta \lambda$ as a function of $y=\left[1-\left(\frac{T}{T_{\mathrm{c}}}\right)^{2}\right]^{-1 / 2}[50]$, indeed for $T / T_{\mathrm{c}}>0.8$ the trend between penetration depth and temperature may be approximated with Equation 7.3. For dirty materials, the variation of the penetration depth tends to be larger than for clean materials. This can be easily inferred just taking into account the Pippard 


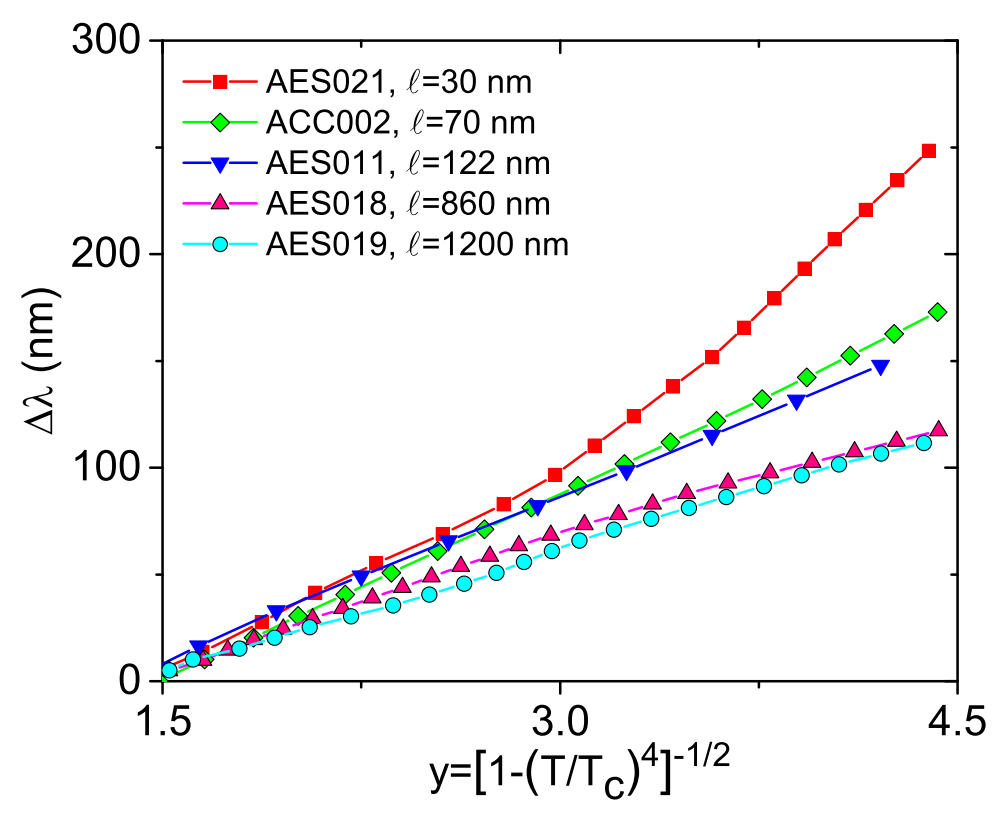

Figure 7.3. Penetration depth variation as a function of $y$ for some of the analyzed cavities, representative of different surface treatments. The connection line between the points serves just as guidance for the eyes.

definition of the penetration depth at $T=0 \mathrm{~K}$ :

$$
\lambda_{0}=\lambda_{\mathrm{L}}\left(1+\frac{\xi_{0}}{\ell}\right)^{1 / 2} .
$$

In Figure 7.3 , the curves of $\Delta \lambda$ as a function of $y$ are reported for some cavities representative of different surface treatments. The trend of mean free path that appears from this graph is in agreement with the results obtained from the interpolation with SRIMP.

Ideally $\Delta \lambda$ as a function of $y$ should be a straight line. Variation from this ideal behavior is observed for some cavities, in particular AES021 and AES018. This may be due to a variation of the mean free path in the material surface, or to the presence of different superconducting phases.

The mean free path of $120^{\circ} \mathrm{C}$ baked cavities is estimated from low energy muon spin rotation (LE- $\mu \mathrm{SR})[70]$ measurements performed on a representative cavity cutout [67], since the fit with SRIMP would have introduced larger error. Indeed, the 


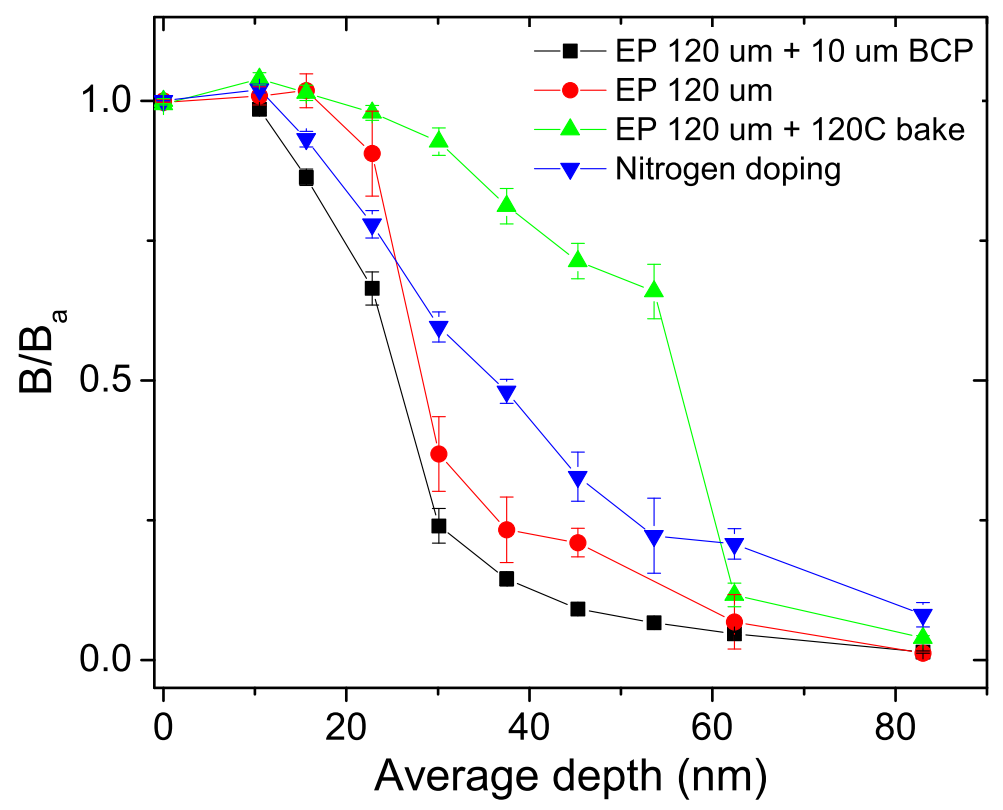

Figure 7.4. Magnetic field penetration as a function of the depth, measured with the $\mu \mathrm{SR}$ technique for different cavity cut-outs. The measured magnetic field $B$ is normalized for the applied field $B_{\mathrm{a}}[7]$.

$120{ }^{\circ} \mathrm{C}$ baking treatment modifies the mean free path only at the very surface of the cavity, for the first $60 \mathrm{~nm}$ or so. For temperatures close to $T_{\mathrm{c}}$ the penetration depth becomes larger than the modified layer, about thousands of nanometers, probing a region which is not representative of the mean free path in the $\mathrm{RF}$ layer at the temperature of interest. In [67] the mean free path of the $120^{\circ} \mathrm{C}$ baked cavity cutout was estimated as between 2 and $16 \mathrm{~nm}$ within the RF layer at $2 \mathrm{~K}$. While the mean free path of the N-doped and EP cut-outs were estimated as $40 \mathrm{~nm}$ and $>400$ $\mathrm{nm}$ respectively.

In Figure 7.4 the magnetic field penetration acquired with LE- $\mu$ SR for different cavity cut-outs, representative of different surface treatments, is shown as a function of the depth [67]. From this graph is clear that the less efficient magnetic field screening is given by the $120{ }^{\circ} \mathrm{C}$ baked cut-out, while cut-outs from EP and BCP cavities show the most efficient screening. The nitrogen-doped cut-out shows instead an intermediate screening behavior. This graph therefore suggests the following as- 
cending order of mean free paths for the studied SRF cavities surface treatments: $120{ }^{\circ} \mathrm{C}$ baking, N-doping, EP.

This mean free path trend suggested from the LE- $\mu \mathrm{SR}$ measurement is in agreement with the trend found by looking at Figure 7.3 and with the values obtained from the interpolation with SRIMP (Table 7.1).

\subsection{Trapped flux sensitivity}

The trapped flux sensitivity $S$ is defined as the trapped flux surface resistance divided by the amount of trapped field:

$$
S=R_{\mathrm{fl}} / B_{\text {trap }}
$$

and indicates the amount of losses generated in the cavity per amount of trapped flux.

In order to clarify the meaning of the trapped flux sensitivity, in Figure 7.5 the Q-factor versus accelerating field curves of two cavities are shown at $1.5 \mathrm{~K}$ after a cooldown with zero trap flux and a cooldown with about $10 \mathrm{mG}$ of trapped flux. Comparing the curves after that the cooldown with zero trap flux, the cavity AES009 show higher value of Q-factors than the cavity AES014. Looking now at the performance of the same cavities after that they trapped about $10 \mathrm{mG}$, in both cases the Q-factor degraded considerably, but now AES014 shows higher Q-factor values than AES009. Indeed, even though they both trapped the same amount of magnetic field, the trapped flux sensitivity of $\operatorname{AES} 014(S \simeq 0.5 n \Omega / m G)$ is considerably lower than the sensitivity of $\mathrm{AES} 009(S \simeq 1.5 n \Omega / m G)$.

The values of sensitivity estimated for all the cavities analyzed are listed in Table 7.1 and are shown as a function of the mean free path in Figure 7.6.

Figure 7.6 shows that the sensitivity has a bell-shaped trend as a function of 


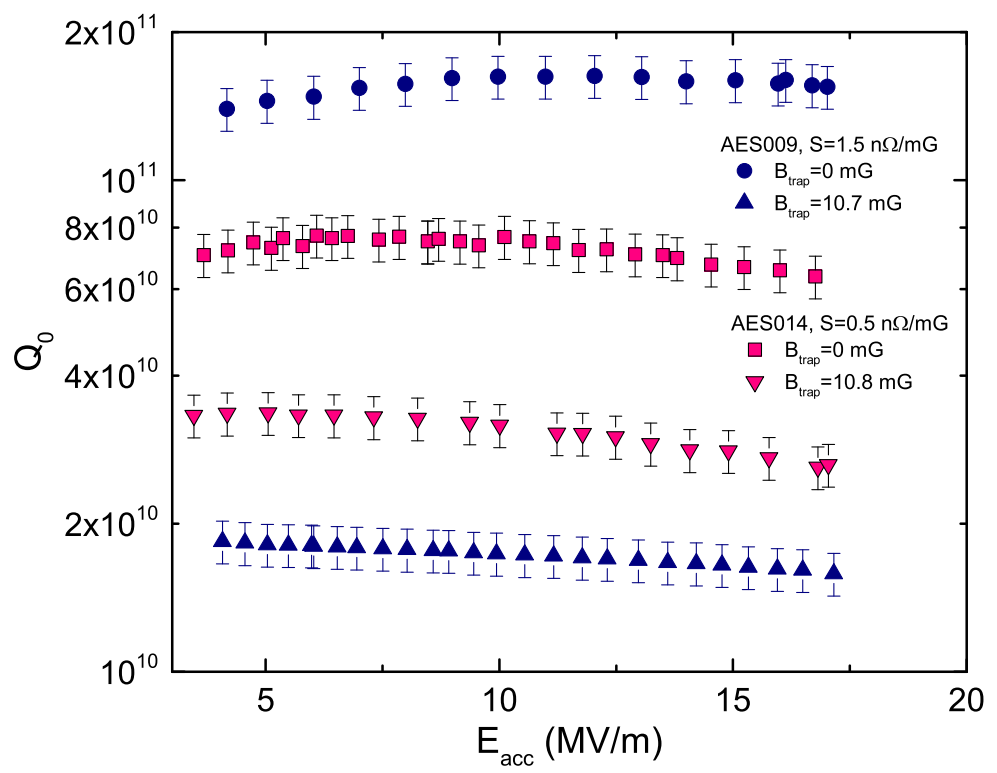

Figure 7.5. Comparison between cavities with same amount of trapped flux and different sensitivity values.

the mean free path. The sensitivity is minimized for both very small $\left(120^{\circ} \mathrm{C}\right.$ bake cavities) and very large (EP and BCP cavities) mean free paths, and it is maximized around $\ell \simeq 70 \mathrm{~nm}$. Taking into account optimal N-doped cavities, when heavilydoped they show the highest sensitivity ( $\ell$ between 70 and $100 \mathrm{~nm}$ ), while the 2/6 recipe, with $\ell$ around $120-180 \mathrm{~nm}$, gives the lowest sensitivity. Doped cavities with $\ell<70 \mathrm{~nm}$ are not optimally doped, and even though they show low sensitivity, they have large intrinsic residual resistance [19], which nullifies the beneficial effect of interstitial nitrogen, as shown in Chapter 4.

The values of sensitivity obtained for EP and BCP cavities are in agreement with previous studies [71], in which sensitivity of $0.35 \mathrm{n} \Omega / \mathrm{mG}$ was measured for a 1.5 GHz cavity made out high purity niobium sheet. Trapped flux sensitivity of $1.3 \mathrm{GHz}$ EP niobium cavities was studied also in Reference [23] in which larger values were found compared to both ours and Reference [71] values.

The experimental data in Figure 7.6 shows some scatter that may be due to 


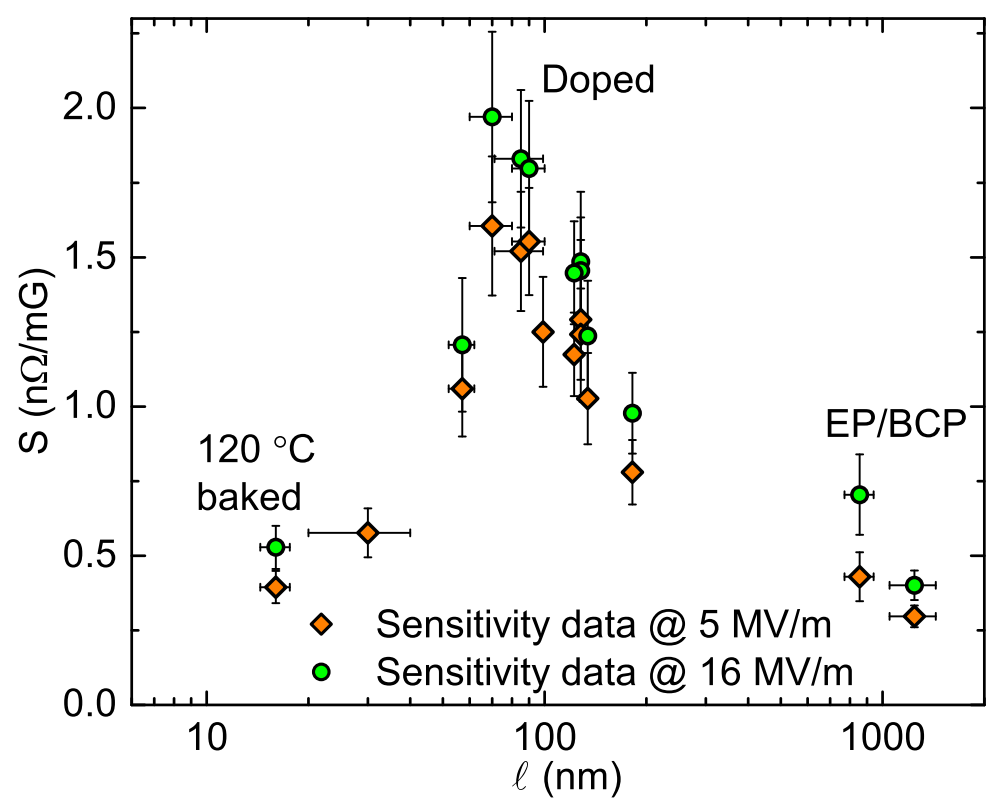

Figure 7.6. Trapped flux sensitivity calculated at $5 \mathrm{MV} / \mathrm{m}$ (orange diamonds) and $16 \mathrm{MV} / \mathrm{m}$ (green dots) as a function of the mean free path [8].

a larger uncertainty on the mean free path values than the error bars since the large number of fit parameters. Differences in terms of pinning force and dimension or position of pinning centers between the analyzed cavities may also increase the data scattering. These parameters indeed seem to play an important role on the trapped flux dissipation [10].

In Figure 7.7 it can be seen that the sensitivity, and therefore the trapped flux surface resistance, increases with the RF field. A field dependence of $R_{\mathrm{fl}}$ was also found studying large grain cavities [72] and niobium on copper thin film cavities [57].

A possible explanation to the trapped flux surface resistance field dependence might be the progressive depinning of vortices from their pinning center, driven by the increasing of the RF field, as hypothesized also in Reference [57].

\subsection{Theoretical explanations of the dissipation due to trap flux}

7.5.1 Vortex local description. In a local description, a vortex may be described 


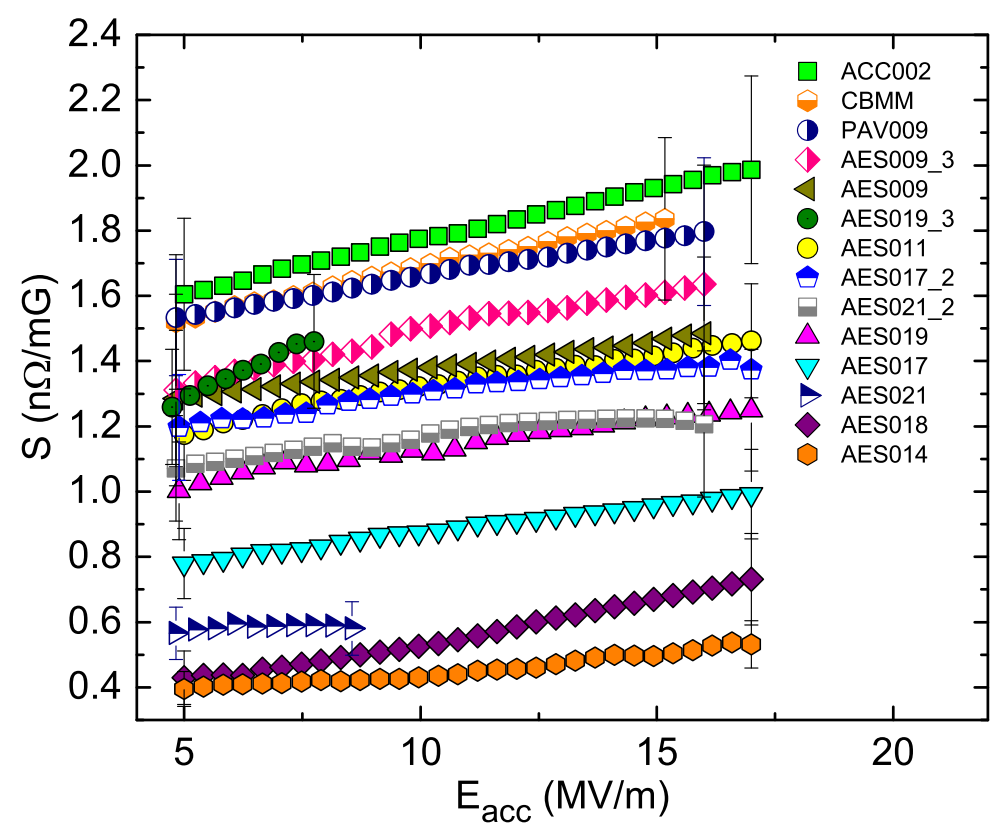

Figure 7.7. Sensitivity dependence on the accelerating field [8].

as a normal conducting core with radius of the order of the coherence length $a \simeq \xi$. The vortex contains a single flux quanta $\Phi_{0}$, and the magnetic field decays over a distance $\lambda_{\mathrm{L}}$ from the center of the core $[73,9]$. In Figure 7.8 , the variation of the energy gap $\Delta$ as a function of the distance $r$ from the center of the vortex is shown. From this graph it can be seen that in the local description $\Delta=0$ at the core of the vortex, indicating its normal-conducting nature.

Once the external field $B_{\text {ext }}$ is trapped over a surface $\Sigma$ of the cavity, it generates $N$ vortices, each containing the fluxoid $\Phi_{0}$ :

$$
\Sigma B_{\mathrm{e}}=N \Phi_{0}
$$

A simple model to calculate losses due to trap flux can be developed considering that the normal-conducting core of the vortex dissipates exactly as the material in the normal state [25]. The trapped flux surface resistance is then calculated as the normal- 


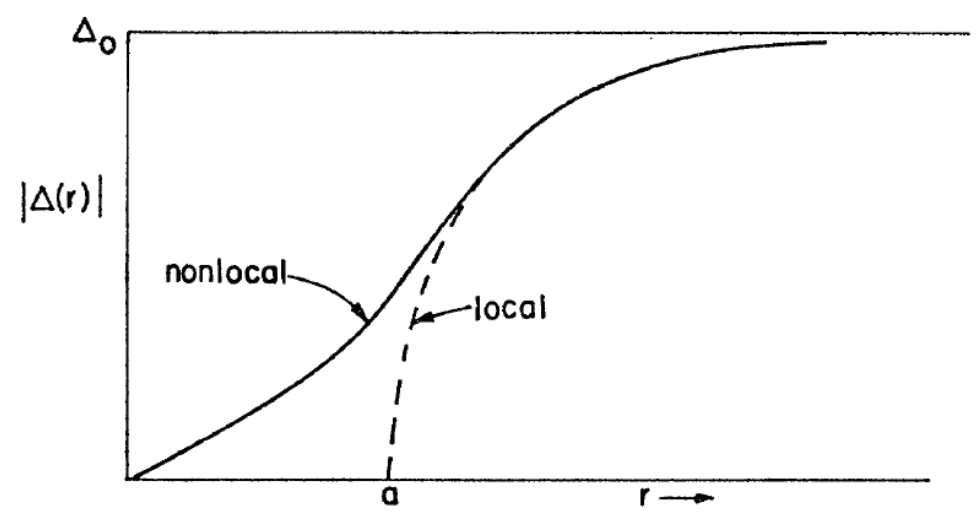

Figure 7.8. Variation of the energy gap $\Delta$ as a function of the distance $r$ from the center of the vortex. The solid line shows the non-local description while the dashed line shown the local description [9].

conducting surface resistance times the fraction of the normal-conducting area:

$$
R_{\mathrm{fl}}=N \frac{\pi a^{2}}{\Sigma} R_{\mathrm{n}}=\frac{\pi a^{2} R_{\mathrm{n}}}{\Phi_{0}} B_{\mathrm{e}}
$$

where $\pi a^{2}$ is the vortex normal-conducting area. Considering that all the external flux is trapped, the sensitivity results:

$$
S=\frac{\pi a^{2} R_{\mathrm{n}}}{\Phi_{0}}
$$

From the Bardeen and Stephen analysis [9] the radius of the normal conducting core $a=\hbar / 2 p_{\mathrm{c}}$ is defined as the distance from the center of the vortex, at which the superelectrons momentum assumes the critical value $p_{\mathrm{c}}$, and the energy gap $\Delta$ goes to zero. In the clean limit $\left(\ell>\xi_{0}\right)$, the critical superelectrons momentum and the vortex radius are:

$$
p_{\mathrm{c}}=\frac{2.178 \hbar}{2 \pi \xi_{0}} \quad, \quad a_{\text {clean }}=1.16 \xi_{0},
$$

while in the dirty limit $\left(\ell<\xi_{0}\right)$ :

$$
p_{\mathrm{c}}=\sqrt{\frac{3 \hbar^{2}}{4 \pi \ell \xi_{0}}} \quad, \quad a_{\text {dirty }}=\sqrt{\frac{\pi}{3} \ell \xi_{0}},
$$

therefore, in this description, the dimension of the vortex's normal-conducting core strictly depends on the mean free path. 
The normal-conducting resistance $R_{\mathrm{n}}$ of niobium at $2 \mathrm{~K}$ and $1.3 \mathrm{GHz}$ is defined by the normal skin effect when $\ell<500 \mathrm{~nm}$ and by the anomalous skin effect when $\ell>500[25]$. The surface resistance in these two limits is:

$$
\begin{gathered}
R_{\mathrm{n}}^{\text {normal }}=\sqrt{\frac{\mu_{0} \omega}{2 \sigma_{\mathrm{n}}}}=\sqrt{\frac{\mu_{0} \omega v_{\mathrm{F}}}{2 n e^{2} \ell}}, \\
R_{\mathrm{n}}^{\text {anomalous }}=\left[\sqrt{3} \pi\left(\frac{\mu_{0}}{4 \pi}\right)^{2}\right]^{1 / 3} \omega^{2 / 3}\left(\frac{\ell}{\sigma_{n}}\right)^{1 / 3} .
\end{gathered}
$$

For the normal skin effect regime, the sensitivity can be calculated as:

$$
\begin{gathered}
S_{\text {clean }}=\frac{\pi}{\Phi_{0}}\left(1.16 \xi_{0}\right)^{2} \sqrt{\frac{\mu_{0} \omega v_{\mathrm{F}}}{2 n e^{2} \ell}} \\
S_{\text {dirty }}=\frac{\pi^{2}}{3 \Phi_{0}} \ell \xi_{0} \sqrt{\frac{\mu_{0} \omega v_{\mathrm{F}}}{2 n e^{2} \ell}},
\end{gathered}
$$

on the other hand, in the anomalous skin regime the sensitivity does not depend on the mean free path in clean limit, while in dirty limit $S_{\text {dirty }} \propto \sqrt{\ell}$.

Since the majority of the studied cavities show $\ell<400 \mathrm{~nm}$, the case of normal skin effect seems to be the most interesting. The sensitivity calculated in the normalskin regime versus $\ell / \xi_{0}$, in the dirty and clean limits, are shown in Figure 7.9 and compared with the experimental data. Since this model is not field dependent, the experimental data of sensitivity shown in Figure 7.9 represents the value at zero field and are extrapolated with a linear interpolation of the sensitivity versus accelerating field curves 7.7 .

Considering that the boundary between clean and dirty limit is around $\ell / \xi_{0} \sim$ 1 , it is possible to notice that this simple model is capable to predict the bell-shape trend of the sensitivity as a function of the mean free path. In the clean limit the sensitivity decreases as the mean free path increases because the resistance of the normal-conducting core decreases for cleaner materials, while the area of the core remains constant. In the dirty limit instead, the sensitivity increases as the mean 


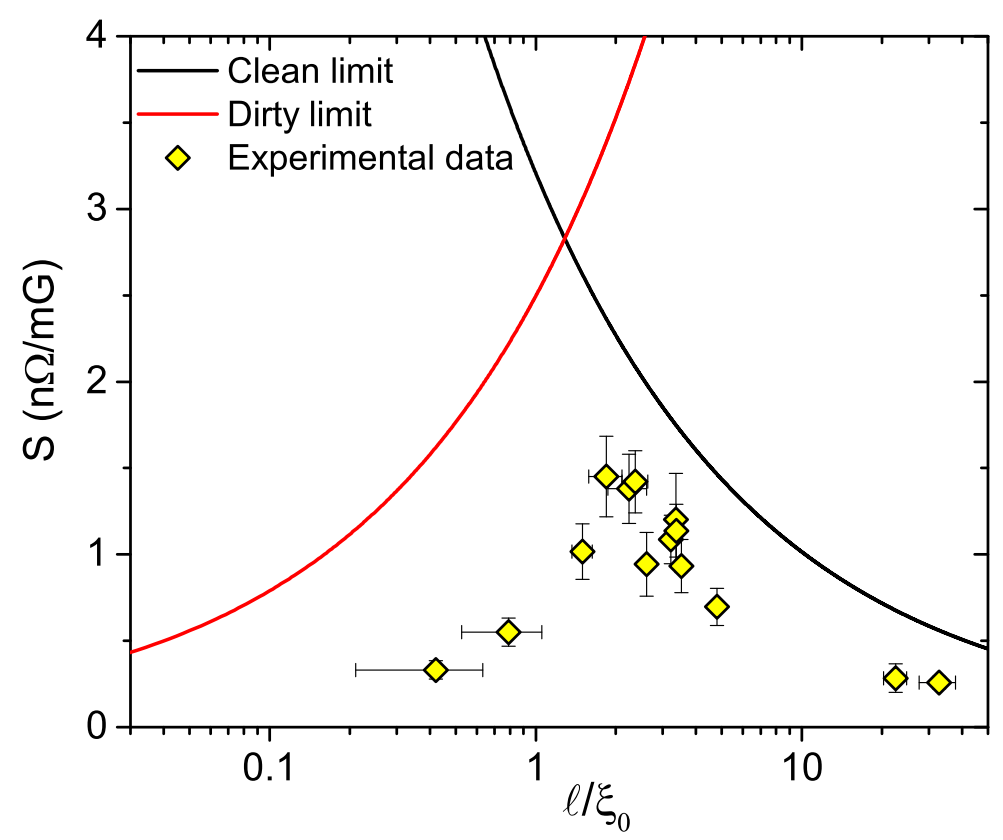

Figure 7.9. Sensitivity as a function of mean free path for the clean (black curve) and dirty (red curve) limits calculated considering dissipation coming from the normal-conducting core of the vortex. The yellow diamonds correspond to the experimental data at zero field.

free path increases because the area of the normal-conducting core increases and this effect prevails on the decreasing of $R_{\mathrm{n}}$.

However, comparing the curves with the experimental data, this model seems to overestimate the sensitivity. The local description of the vortex is probably a too crude approximation and a non-local description is needed in order to take into account that the normal-conducting core is actually a zero-singularity point of the density of Cooper pairs. Using this description the dissipation is introduces only from the vortex oscillation driven by the RF field.

7.5.1.1 Vortex non-local description. The structure of an isolated vortex was described by Abrikosov after solving the Ginzburg-Landau equations for $\Psi(r)$ and $h(r)$, where $\Psi(r)$ is the order parameter and $h=H / H_{\mathrm{c} 1}$ is the reduced magnetic field. In Figure 7.10 it is possible to see that $\Psi(r)$ is constant at $r \rightarrow \infty$ and rapidly 


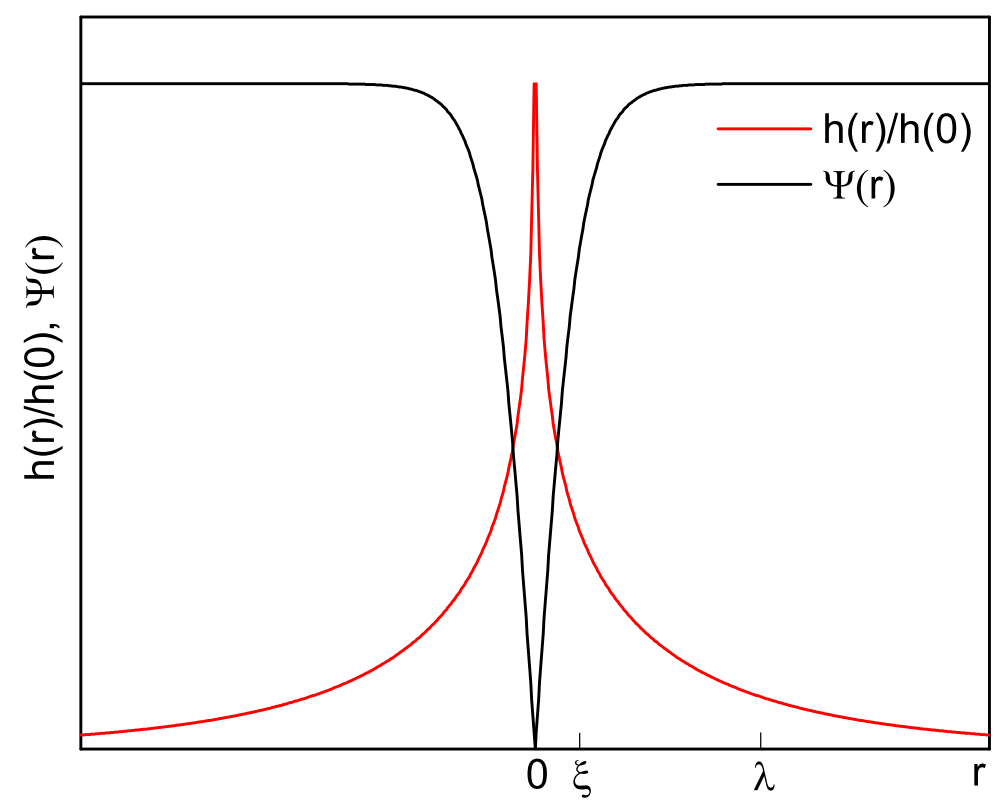

Figure 7.10. Normalized reduced magnetic filed $h(r) / h(0)$ and order parameter $\Psi(r)$ as a function of the radial distance from the center of vortex $r$ in the Abrikosov vortex description.

decay to zero when $r \approx \xi, h(r)$ instead is maximum at the center of the vortex and decays approaching to zero for $r \rightarrow \infty$. For simplicity, in Figure 7.10, $h(r)$ is calculated using the Clem model which is an approximation valid for high $\kappa_{G L}$ [36].

Therefore, in this non-local description, it is not possible to define a normalconducting core with a finite radius.

In this case the dissipation is instead related to the vortex oscillation due to the Lorentz force $\bar{J} \times \Phi_{0}$ that acts on the vortex whenever a current density $\bar{J}$ flows in the superconductor. In case of SRF cavities, the current density $\bar{J}$ flows at the surface of the cavity induced by the RF field.

The vortex movement can be described, as first proposed by Gittleman and Rosenblum [74], as a damped oscillator:

$$
M \frac{\partial^{2} x}{\partial t^{2}}=f_{\mathrm{L}}+f_{\mathrm{v}}+f_{\mathrm{p}},
$$

where $M$ is the inertial mass of the vortex [9], $f_{\mathrm{L}}$ is the Lorentz force per unit of 
length:

$$
f_{\mathrm{L}}=\left|\bar{J} \times \Phi_{0} \hat{u_{\mathrm{n}}}\right|=j_{0} \Phi_{0} \sin \theta e^{i \omega t-z / \lambda}
$$

where $\hat{u_{\mathrm{n}}}$ is the unit vector normal to the RF surface, $\theta$ is the angle between $\bar{J}$ and $\hat{u_{\mathrm{n}}}, \omega$ is the angular frequency, $z$ is the depth in the material and $\lambda$ is the penetration depth; $f_{\mathrm{v}}=-\eta \dot{x}$ is the viscous force where $\eta$ is the viscous drag coefficient [9]:

$$
\eta=\frac{\Phi_{0} B_{\mathrm{c} 2}}{\rho_{\mathrm{n}}},
$$

where $\rho_{\mathrm{n}}$ is the normal state resistivity and $B_{c 2}$ the upper critical field; the pinning force $f_{p}$ can be assumed, in first approximation, as a simple elastic restoring force:

$$
f_{\mathrm{p}}=-\frac{\partial}{\partial x} U_{\mathrm{p}}=-p x
$$

where $U_{\mathrm{p}}$ is the pinning potential and $p$ is the pinning constant.

The vortex dissipation was recently calculated by Checchin et al. [10] by solving the vortex motion equation, considering a bi-dimensional Lorentzian where more than one pinning sites are considered along the $z$-direction. By means of a parabolic approximation along the direction of oscillation $(x)$, the pinning potential is defined as parabolic along the direction of oscillation and Lorentzian along $z$. The equation of motion can in this way be solved analytically.

This model predicts that for large values of mean free path the dissipation is described by the flux flow regime while for low mean free path the dissipation is governed by the pinning in the material, in the so-called pinning regime.

In the flux flow regime the pinning force can be neglected, therefore the main force acting on the vortex is the viscous drag force. Under this assumption the resistivity is purely real and equal to:

$$
\rho \simeq \frac{\Phi_{0} \sin ^{2} \theta}{\pi \xi_{0}^{2} \eta}
$$




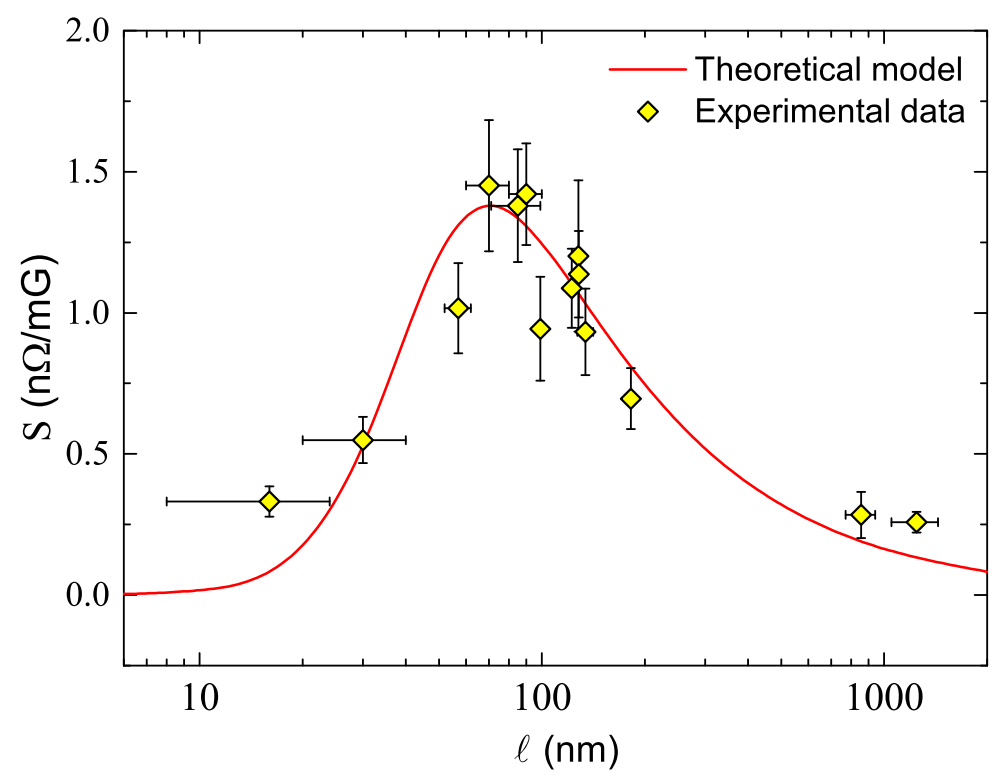

Figure 7.11. Sensitivity as a function of the mean free path calculated considering dissipation coming from the vortex oscillation due to RF field [10] (red curve) and comparison with experimental data at zero field (yellow diamonds).

which is the same found in References [75, 76]. This results highlights that the dissipation in this regime depends only on the mean free path through the viscous coefficient $\eta$. Since $\eta$ increases due to the decreasing of $\rho_{\mathrm{n}}$, the resistivity decreases as the mean free path increases .

At small value of mean free path the viscous drag force becomes negligible, while the pinning force is large. Such condition is called pinning regime and the complex resistivity is defined as:

$$
\rho=\frac{\omega \Phi_{0}^{2} \sin ^{2} \theta}{\pi \xi_{0}^{2} p^{2}}[\eta \omega+i p] .
$$

As the mean free path decreases, the real part of the resistivity decreases as well and becomes negligible for very low value of mean free path. In this limit the resistivity is purely imaginary. 
Once the resistivity is known, the surface impedance is calculated as:

$$
Z=\frac{E_{\mathrm{x}}(0)}{\int_{0}^{\infty} j_{\mathrm{x}}(z) d z}=\frac{1}{\int_{0}^{\infty} \frac{e^{-z / \lambda}}{\rho(z, \ell)} d z}
$$

The sensitivity is then defined as the real part of the surface impedance divided by the trapped field. The result obtained of the sensitivity as a function of the mean free path is shown in Figure 7.11. The graph shows that the model is adherent to the experimental data at zero field.

Another model was developed by Gurevich and Ciovati [11], taking into account the non-local description of the vortices. They used an alternative approach in which the pinning force is not included in the equation of motion:

$$
\eta \frac{\partial u}{\partial t}=\hat{\epsilon} \frac{\partial^{2} u}{\partial z^{2}}+j_{0} \Phi_{0} e^{i \omega t-z / \lambda}
$$

where $z$ is the axis perpendicular to the surface, $u(z, t)$ is the vortex displacement parallel to the surface and $\epsilon$ is the vortex line tension in an uniaxial superconductor. The pinning condition is instead introduced by applying the following boundary conditions to the equation of motion (Equation 7.23):

$$
\frac{\partial u(0)}{\partial z}=0 \quad, \quad u(d)=0
$$

where $d$ is the distance of the pinning center from the surface. These boundary conditions set that at one end the vortex has to be perpendicular at the surface, while the other end is fixed by the pinning center.

This approach allows to solve the vortex equation of motion analytically, and to calculate the dissipated power, from which it is possible to calculate the trapped flux surface resistance, as:

$$
P=-\frac{\omega J_{0} \Phi_{0}}{2} \int_{0}^{d} u(z, \omega) e^{-z / \lambda} d z=\frac{R_{f l} H^{2}}{2} .
$$




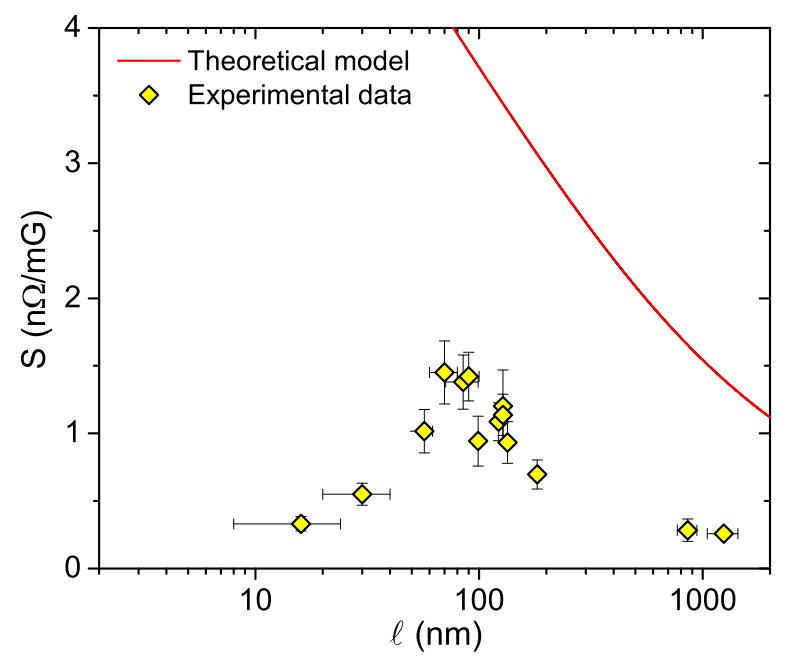

Figure 7.12. Sensitivity as a function of mean free path calculated from the Gurevich and Ciovati model [11]. The yellow diamonds correspond to the experimental data at zero field.

In the range of frequency interesting for SRF applications, $R_{f l}$ results [11]:

$$
R_{f l}=\frac{B_{e}}{B_{c}}\left(\frac{\mu_{0} \rho_{n} \omega}{1+\frac{2}{\Gamma} \ln \left(\Gamma \kappa_{G L}\right)}\right)^{1 / 2},
$$

where $B_{e}$ is the external field, $\Gamma=\lambda_{c} / \lambda$ is the anisotropic parameter and $\lambda_{c}$ the penetration depth along the z-axis.

The sensitivity can be therefore calculated from Equation 7.26. Since niobium has a body centered cubic lattice the anisotropy effects may be disregarded and it is possible to set $\Gamma=1[77]$. The normal resistance $\rho_{n}$ is calculated as before from the Drude model and $\lambda$ and $\xi$ defined in the GL constant $\kappa_{G L}$ are calculated from the Pippard's definitions:

$$
\lambda=\lambda_{L} \sqrt{1+\frac{\xi_{0}}{\ell}} \quad, \quad \frac{1}{\xi}=\frac{1}{\xi_{0}}+\frac{1}{\ell}
$$

while the critical field does not depends on the mean free path and it is set as $B_{c}=180$ $\mathrm{mT}[78]$. 
The curve of sensitivity as a function of the mean free path obtained from this model is shown in Figure 7.12, where it is also compared with the experimental data. From this graph it is possible to notice that this model is capable to explain only the decreasing of the sensitivity as a function of the mean free path that appears for large $\ell$. The values of sensitivity obtained with this model are overestimated in comparison with the experimental data.

From this section it is possible to conclude that the experimental data of sensitivity as a function of mean free path can be described by the model made by Checchin et al. [10]. In agreement with this model, the motion of the vortices is subject to viscous drag and pinning, which introduce dissipation. The viscous drag prevails for very clean superconductors, while the pinning prevails for low mean free path values. Interesting is also that the model predicts that the trapped flux surface resistance is proportional to $\omega^{2}$, in the pinning regime (Equation 7.21). This means that for low values of mean free path the sensitivity increases with the increasing of the resonance frequency. It is therefore expected that nitrogen-doped cavities with low resonance frequency, as $650 \mathrm{MHz}$ elliptical cavities, show lower values of trapped flux sensitivity. Another interesting point is that in the pinning regime the resistance is proportional to $p^{-2}$ (Equation 7.21). Therefore, for dirty materials, the sensitivity is minimized when defects define strong pinning centers.

\subsection{BCS surface resistance analysis}

The BCS surface resistance at $2 \mathrm{~K}$ is extrapolated after the cooldown with no flux trapped, as the difference between the $R_{\mathrm{s}}$ measured at $2 \mathrm{~K}$ and at $1.5 \mathrm{~K}$ (Equation 7.2).

As it can be seen from Figure 7.13, the BCS surface resistance of N-doped cavities decreases as a function of the accelerating field. On the other hand, for 


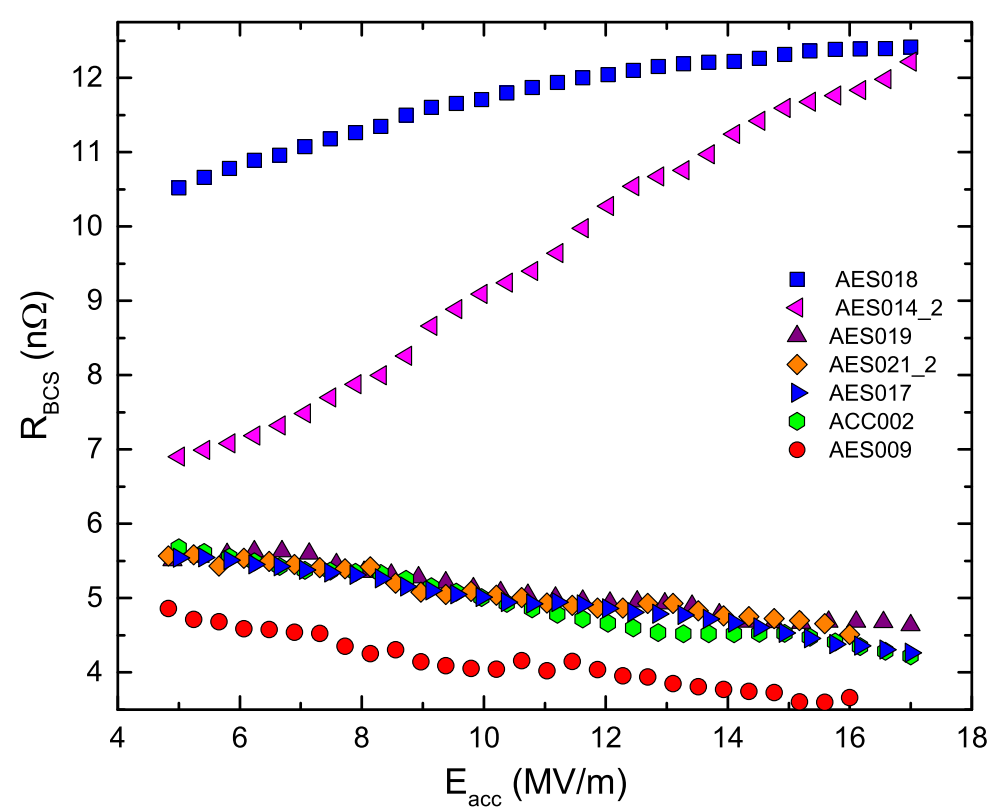

Figure 7.13. 2 K BCS surface resistance as a function of the accelerating field of some of the cavities analyzed.

standard niobium cavities, both EP and $120^{\circ} \mathrm{C}$ baked cavities, $R_{\mathrm{BCS}}$ increases as a function of the accelerating field. The peculiar decreasing of $R_{\mathrm{BCS}}$ versus $E_{\mathrm{acc}}$ is shown only in N-doped cavities, and represent the origin of the typical anti-Q-slope [19].

In order to further understand the physical origin of such small values of $R_{\mathrm{BCS}}$ at medium field, $R_{\mathrm{BCS}}$ is analyzed as a function of the mean free path.

$R_{\mathrm{BCS}}$ as a function of the mean free path is shown in Figure 7.14. The upper graph shows the results obtained at low field $(5 \mathrm{MV} / \mathrm{m})$, while the bottom graph shows the results at medium field $(16 \mathrm{MV} / \mathrm{m})$.

Regarding the EP cavities, the mean free path is calculated for one cavity (AES018) and the other one is fixed at the same value, assuming that they should show very similar values. For one N-doped cavity (AES005) the mean free path is directly measured on a cavity cut-out with LE- $\mu \mathrm{SR}[7]$. 


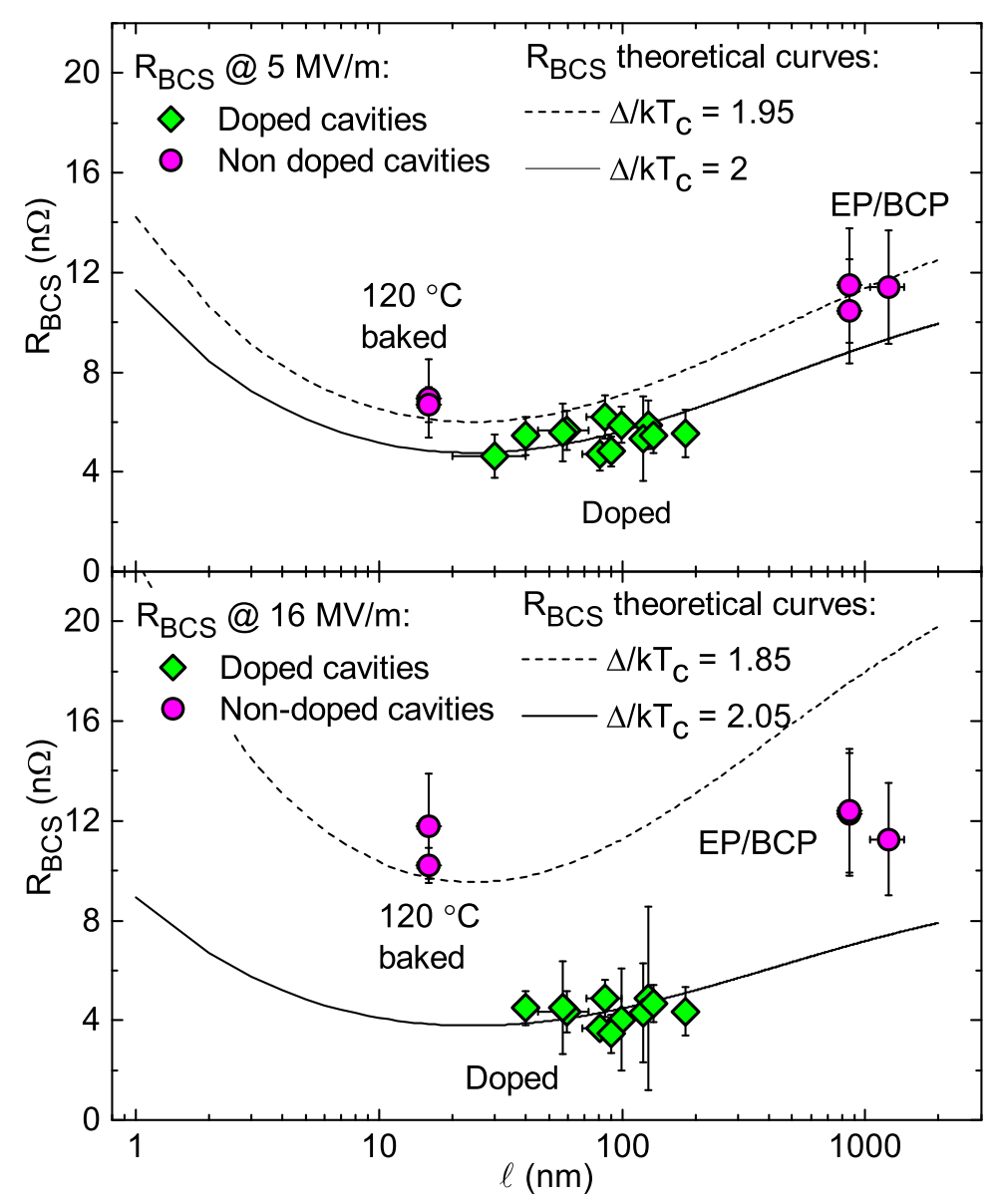

Figure 7.14. $2 \mathrm{~K}$ BCS surface resistance as a function of mean free path, at $5 \mathrm{MV} / \mathrm{m}$ (upper graph) and $16 \mathrm{MV} / \mathrm{m}$ (lower graph). The green diamonds represent doped cavities, while the pink circles represent non doped cavities [8].

The green diamonds represent doped cavities, while the pink circles are niobium cavities with different standard treatments $\left(120{ }^{\circ} \mathrm{C}\right.$ bake, BCP and EP). The black curves are theoretical curves of $R_{\mathrm{BCS}}$ versus mean free path estimated using SRIMP [2] for different reduced energy gap values.

In both field regimes, doped cavities show lower values of $R_{\mathrm{BCS}}$ than non-doped cavities, proving that $R_{\mathrm{BCS}}$ is lowered with the introduction of interstitial impurities, as predicted by the Mattis and Bardeen theory. At medium field the difference in $R_{\mathrm{BCS}}$ between doped and non-doped cavities is maximized due to the opposite trend of this surface resistance contribution as a function of the accelerating field. 
The values of $R_{\mathrm{BCS}}$ obtained for all the cavities analyzed cannot be described by one single theoretical curve, both at low and medium field, suggesting that the mean free path is not the only parameter changing with the introduction of impurities. Following this hypothesis, one of the other parameters on which the BCS surface resistance depends on $\left(\lambda_{\mathrm{L}}, \xi_{0}, \Delta, T_{\mathrm{c}}\right)$ is changing as well. In the low field case,

fixing all the other parameters and changing the reduced energy gap $\Delta / k T_{\mathrm{c}}$, the $120^{\circ} \mathrm{C}$ baked, BCP and EP cavities are interpolated with $\Delta / k T_{\mathrm{c}}=1.95$, while doped cavities are better interpolated setting $\Delta / k T_{\mathrm{c}}=2$. At medium field, the difference is even larger being $\Delta / k T_{\mathrm{c}}=1.85$ for $120^{\circ} \mathrm{C}$ baked cavities and $\Delta / k T_{\mathrm{c}}=2.05$ for doped cavities. For BCP and EP cavities, $\Delta / k T_{\mathrm{c}}$ is probably slightly larger than the value assumed for $120^{\circ} \mathrm{C}$ baked cavities.

This suggests a possible field dependence of the gap. Comparing the upper and lower graph of Figure 7.14, for doped cavities $\Delta / k T_{\mathrm{c}}$ increases passing from 5 to $16 \mathrm{MV} / \mathrm{m}$. This variation may be the reason why $R_{\mathrm{BCS}}$ decreases with the $\mathrm{RF}$ field for doped cavities. Increasing of the energy gap with the RF field has been measured in the past [79], and in that case the enhancement of superconductivity was attributed to non-equilibrium effects $[80,81]$. In the Eliashberg theory the minimum frequency at which non-equilibrium effects may be visible depends on the inelastic collision time of quasi-particles scattering with phonons $\tau_{\mathrm{E}}$ and for niobium this minimum frequency is around $15 \mathrm{GHz}$ [80]. One possibility is therefore that the introduction of interstitial nitrogen inside the niobium lattice, modifies this parameter, decreasing the cut-off frequency at values lower that $1.3 \mathrm{GHz}$.

\subsection{The best surface treatment for SRF cavities}

Adding together the measured values of $R_{\mathrm{BCS}}$ and sensitivity, it is possible to reveal which treatment is capable to give the highest Q-factors taking into account different amount of trapped flux. 
In order to visualize that, the Q-factor is calculated, for each treatments among EP, $120^{\circ} \mathrm{C}$ baking and $\mathrm{N}$-doping, as follows:

$$
Q_{0}=G /\left(R_{\mathrm{BCS}}+S \times B_{\text {trap }}+R_{0}\right),
$$

where the values of $B_{\text {trap }}$ ranges from 0 to $20 \mathrm{mG}$.

The intrinsic residual resistance $R_{0}$ depends on many parameters, some related to the surface treatments and others related to the bulk itself [45]. $120{ }^{\circ} \mathrm{C}$ baked cavities usually show value of $R_{0}$ greater than both EP and optimized N-doped cavities $[19,82]$. Because of that, the calculation was performed assuming as intrinsic residual resistance: $R_{0}=4 \mathrm{n} \Omega$ for the $120{ }^{\circ} \mathrm{C}$ baked cavity and $R_{0}=2 \mathrm{n} \Omega$ for both $\mathrm{EP}$ and N-doped cavities, which are common values found for these treatments.

In Figure 7.15 is shown the resulting Q-factors, at $16 \mathrm{MV} / \mathrm{m}$ and $2 \mathrm{~K}$, versus the mean free path. For simplicity one point is shown for the $120^{\circ} \mathrm{C}$ baking and EP treatments, and two points for the N-doping treatment. The two points are chosen as indicating the best and the worst scenario. The curves of Q-factor versus mean free path are calculated considering different values of trapped field. From the graph it is possible to notice that when there is no field trapped, the N-doping treatment gives the highest Q-factors, which are more than doubled compared with the standard niobium cavities. In addition, it is possible to notice that under this condition the highest Q-factor is actually given by heavily doped cavities.

As soon as some field is trapped in the cavity, the highest Q-factors are given by light N-doping treatment. The Q-factors of heavily-doped cavities become lower than the ones obtainable with standard treatments after that the trapped field is around $10 \mathrm{mG}$. For the best case of $\mathrm{N}$-doping treatment instead, the Q-factor is the highest as long as the trapped field in the cavity is about $20 \mathrm{mG}$.

Therefore, Figure 7.15 highlight that, even though N-doped cavities have larger 


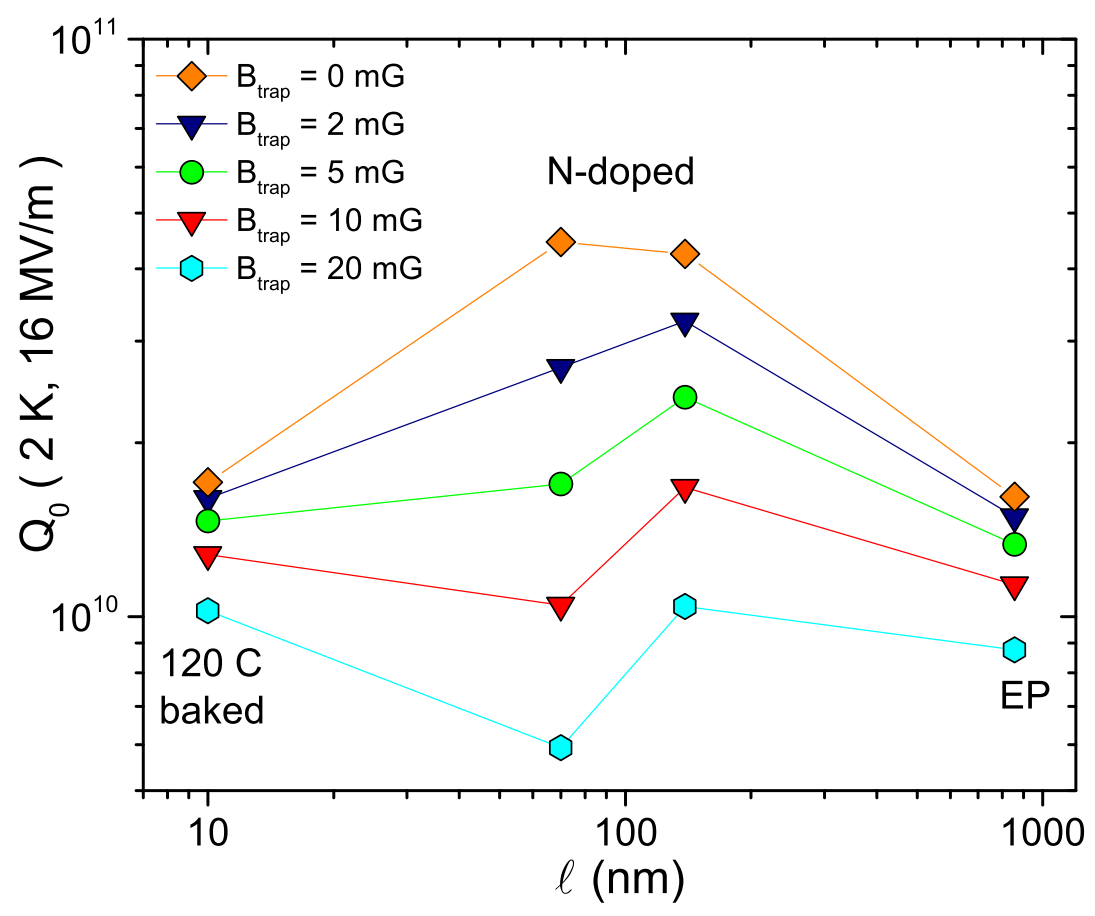

Figure 7.15. Q-factor at $2 \mathrm{~K}$ and $16 \mathrm{MV} / \mathrm{m}$ as a function of the mean free path. In order to make the graph as clear as possible, for the N-doped cavities only two points are shown. These two points represent the best and the worst case scenario among all the N-doped studied cavities [8].

sensitivity to trapped flux, optimizing the mean free path it is still possible to maintain the high Q-factors, even in presence of trapped flux.

In order to further clarify the range of trapped flux in which the N-doping is capable to give the highest Q-factor, in Figure 7.16 the Q-factor is shown as a function of $B_{\text {trap }}$. In this graph the $2 / 6 \mathrm{~N}$-doping treatment is compared with the two standard niobium treatments. Among the N-doped cavities, the $2 / 6 \mathrm{~N}$-doping is indeed the recipe of greatest interest for high-Q application, since it shows the best compromise between $R_{\mathrm{BCS}}$ and sensitivity values exploited so far. From this graph it is clear that the 2/6 N-doped cavity shows the highest values of Q-factor as long as the trapped field is lower than $10 \mathrm{mG}$, i.e. within the range of realistic values of remnant magnetic field achievable in modern cryomodules. 


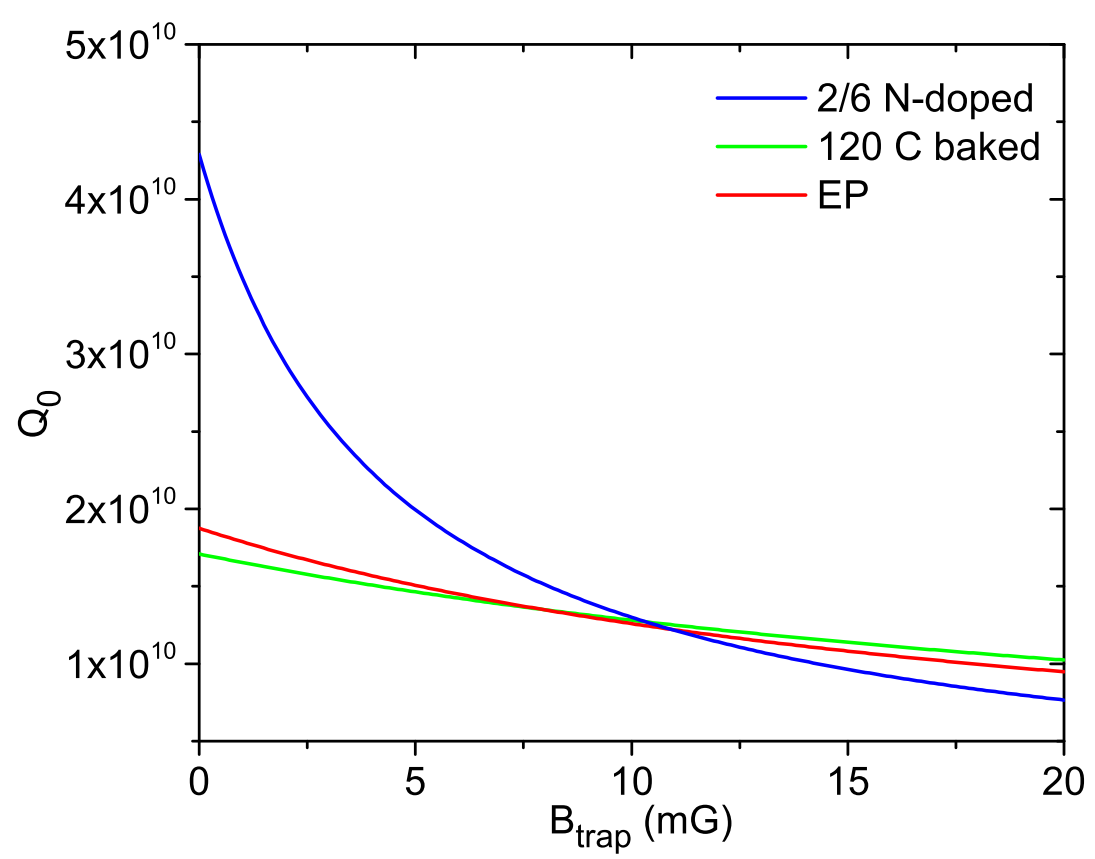

Figure 7.16. Q-factor at $2 \mathrm{~K}$ and $16 \mathrm{MV} / \mathrm{m}$ as a function of the trapped field for a $120^{\circ} \mathrm{C}$ bake, EP and 2/6 N-doped cavity.

For example, using the $2 / 6 \mathrm{~N}$-doping treatment it is possible to fully trap in the cavity 2.5 mGauss of external magnetic field and reach the LCLS-II specification $\left(Q_{0}=2.7 \cdot 10^{1} 0\right.$ at $2 \mathrm{~K}$ and $\left.16 \mathrm{MV} / \mathrm{m}\right)$. Since the situation in which the magnetic field is fully trapped in the cavity is the worst scenario that may happen during the cavity cooldown, interesting is to analyze the level of remnant magnetic field that the cryomodule may sustain as a function of the flux expulsion level. In Figure 7.17 the percentage of expelled flux needed to maintain the trapped flux at $2.5 \mathrm{mG}$ is shown as a function of the remnant magnetic field $\left(B_{\mathrm{amb}}\right)$ in the cryomodule. For example, with $50 \%$ of flux expulsion the field may be up to $5 \mathrm{mG}$, while with $75 \%$ may be up to $10 \mathrm{mG}$, and so on.

\subsection{Conclusions}

This chapter provides insight on how the global surface resistance, and hence the Q-factor, vary with the different surface treatments. Both the BCS and the 


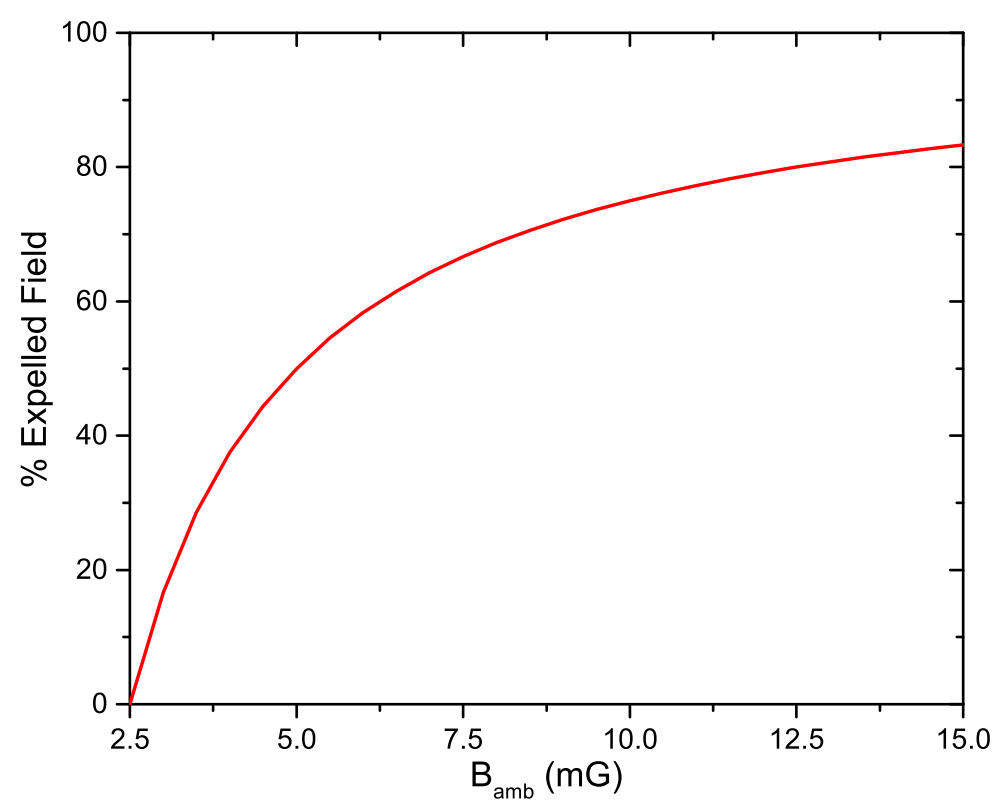

Figure 7.17. Percentage of expelled flux needed to reach LCLS-II specification with $2 / 6 \mathrm{~N}$-doped cavities as a function of the remnant magnetic field in the cryomodule.

trapped flux surface resistance contributions have been indeed analyzed as a function of the mean free path.

It was found that the trapped flux surface resistance normalized per amount of trapped flux, i.e. the sensitivity, has a bell-shaped behavior as a function of the mean free path. Consequence of this behavior is that $\mathrm{N}$-doped cavities have larger values of sensitivity and, therefore, larger losses due to trap flux. In order to minimize this kind of losses in $\mathrm{N}$-doped cavities, light treatments are needed, as for example, the 2/6 N-doping treatment.

In order to understand the physics behind the trapped flux dissipation, some model have been described. One simple model consider the dissipation as coming from the normal-core of the vortex. This model is capable to explain the bell-shaped trend of the sensitivity as a function of the mean free path. For large mean free path, the sensitivity decreases with the mean free paths because of the lowering of the normal state resistivity. On the other hand, for low value of mean free path, the 
opposite trend is given by the increasing of the area of the vortex core moving toward larger value of mean free path. However, the values of sensitivity that results from this simple model are overestimated.

It was shown that a better approach takes into account that the dissipation is due to the vortex movement under the RF field, using a non-local description of the vortex. The model developed by Checchin et al. [10], is capable to well describe the experimental data and it suggests that the bell shape trend is a consequence of the damping of the vortex movement due to: the material viscosity for large mean free path, and the pinning for low mean free path.

In addition, the physics behind the lowering of the BCS surface resistance with the accelerating field was analyzed in this chapter. Comparing experimental data with theoretical curves, it was shown that the introduction of impurities in the material causes not only the modification of the mean free path, but also the increasing of the reduced energy gap $\Delta / k T_{\mathrm{c}}$ at medium field. The anti Q-slope of $\mathrm{N}$-doped cavities may be therefore explained as the decreasing of the energy gap with the field caused by microwave-driven non-equilibrium effects.

Concluding, from a practical point of view, these results are of crucial importance in order to identify the best surface treatment that allows to reach the highest Q-factors, taking into account all the surface resistance contributions and their dependencies on mean free path, RF field and DC external magnetic field. It was verified that the 2/6 N-doping treatment is capable to give the highest Q-factor values, as long as the magnetic field fully trapped in the cavity is lower than $10 \mathrm{mG}$. This implies that: i) it is crucial to optimize magnetic flux expulsion during the cavity superconducting transition and ii) high Q-factors SRF cryomodules need sophisticate magnetic field screening in order to obtain very low values of remnant magnetic field. 


\section{CHAPTER 8 \\ CONCLUSIONS}

In this thesis work different aspects of superconducting accelerating cavities are studied in order to understand how quality factors can be maximized, from the vertical test measurement to a cryomodule-like environment.

In order to maximize quality factors in superconducting cavities, it is necessary to minimize all the surface resistance components, that are: the BCS surface resistance, the trapped flux surface resistance and the intrinsic residual resistance.

The intrinsic residual resistance does not have a particular trend as a function

of the mean free path. As a matter of fact, the intrinsic residual resistance of $120^{\circ} \mathrm{C}$ baked cavities is in general larger than EP and N-doped cavities. In Chapter 4 it was shown that also heavily doped cavities may present larger values of intrinsic residual resistance.

The BCS and the trapped flux surface resistance strongly depend on the cavity surface treatment. Both these contributions were therefore studied for several different cavities as a function of the mean free path. The mean free path is indeed the parameter that correlates with the impurities introduced in the cavities surface during each treatment.

In Chapter 7 it was shown that the BCS surface resistance of N-doped cavities shows two peculiarity:

- it is really close to the theoretical minimum of the BCS surface resistance versus mean free path curve.

- it decreases as a function of the accelerating field, while for standard niobium cavities it increases. 
Analyzing the $R_{B C S}$ versus mean free path trend for low and medium accelerating field values, and comparing the experimental points with theoretical curves, it was found that the lower values of $R_{B C S}$ of $\mathrm{N}$-doped cavities at medium field may be due to an increasing of the reduced energy gap $\Delta / k T_{c}$ with the RF field.

When looking for the best surface treatment, it is really important to take into account the possibility to have field trapped in the cavity walls. Indeed, when the cavity is inside a cryomodule, there will be always some remnant magnetic field presents during the cavity cooldown that can be trapped. In Chapter 7 it was shown that N-doped cavities show larger trapped flux sensitivity than standard niobium cavities, meaning that they have larger trapped flux surface resistance when the same amount of magnetic field is trapped in the cavity walls. It was found that the curve of sensitivity as a function of the mean free path has a maximum around $70 \mathrm{~nm}$, which is a typical mean free path value of heavily doped cavities. Both EP and $120{ }^{\circ} \mathrm{C}$ baked cavities stay instead at the tail of this curves, showing low dissipation due to trapped flux. Consequently, in order to minimize the trapped flux surface resistance contribution, light N-doping treatments, like the 2/6 N-doping, are needed.

Since the BCS and the trapped flux surface resistance have almost opposite behavior as a function of the mean free path, the surface treatment capable to maximize Q-factors is the one that show the best compromise between these two contributions. Among all the surface treatments studied, the $2 / 6 \mathrm{~N}$-doping treatment is the one that gives the best compromise. Precisely, it was shown that the 2/6 N-doping treatment gives the highest Q-factor values as long as the trapped field is lower than $10 \mathrm{mG}$.

Another method to decrease the trapped flux surface resistance is to decrease the amount of magnetic field trapped in the cavity wall. In Chapter 5 the cavity cooldown is studied in order to understand how the fraction of external magnetic field which is actually trapped in the cavity can be minimized. This study was 
performed cooling down the cavity in a horizontal configuration that resembles the geometry of cavities cooled in a cryomodule. It was found that, as for the vertical cooldown configuration, fast cooldowns generate large thermal gradients along the cavity that helps the magnetic flux expulsion during the superconducting transition. However, during the vertical cooldown large thermal gradients are easy to generate during the SC transition at the cavity equator, which is the area of the cavity in which losses are concentrated. While, during the horizontal cooldown, it is more difficult to maintain large thermal gradient during the transition of the whole the cavity equator, increasing the probability of trapping flux.

In addition, in Chapter 5 it was also found that the orientation of the external magnetic field matters in the case of horizontal cooldown. In particular, when the cavity is cooled fast in the horizontal configuration, with orthogonal magnetic field applied, this field is trapped on top of the cavity equator, increasing the dissipation and causing a local temperature rising. This phenomenon was called flux-hole, since the top of the equator acts as a hole in the superconductor in which the magnetic field is free to penetrate.

Because of the importance of the cooldown in determining the amount of magnetic field trapped in the cavity and, therefore, the trapped flux surface resistance contribution, the physics behind the magnetic flux expulsion during the SC transition was studied in Chapter 6. The magnetic flux expulsion, i.e. the flushing out of vortices from the superconducting cavities, is found to be consequence of the thermodynamic force which moves vortices from the Meissner state to the mixed state in order to minimize the Gibbs free energy. Moreover, in order to take into account that different material properties may cause different flux expulsion behavior, a statistical distribution of pinning centers was introduced as distribution of probability density of trapping vortices when a critical thermal gradient is reached. In order to achieve 
a complete flux expulsion situation, it is necessary to reach the maximum value of the critical thermal gradient of the probability density function that describes the pinning centers distribution. Comparing this model with experimental data it was found that most of the studied cavities are actually described by a double pinning centers distributions, suggesting that there are two main group of defects that act as a pinning centers.

In Chapter 6 the different dynamics of slow and fast cooldown was studied by means of a T-map system. This allowed to monitor and map the temperature around the whole cavity during both the fast and the slow cooldown. The temperature maps revealed that during the fast cooldown the SC transition starts from the bottom and propagates through the top of the cavity, really sharply, defining the large thermal gradients needed for the flux expulsion. On the other hand, the temperature maps acquired during the slow cooldown reveals how the temperature is homogeneous all around the cavity, minimizing the thermal gradients. The SC transition starts and propagates randomly around the cavity, therefore at the end of the cavity transition several normal-conducting regions are randomly distributed among the cavity, surrounded by the superconducting phase. The vortices are therefore concentrated in this region and the magnetic field can not be expelled anymore even after that these areas become superconducting.

Summarizing, during the fast cooldown, the sharp interface between the SC and NC phases promotes the generation of large thermal gradient that facilitates the vortices movement from the bottom to the top of the cavity. If the cavity is vertically cooled, the magnetic field can be successfully expelled from the cavity once it become completely superconducting. The same happens if the cavity is horizontally cooled in presence of axial magnetic field. When, instead, the cavity is horizontally cooled in presence of orthogonal magnetic field, the vortices that are moved toward 
the top of the cavity, reaching the top of the equator, can not be expelled anymore because they do not have any energetically favorable path to follow. Similarly, during the slow cooldown the poor thermal gradients move vortices toward the normalconducting area. These vortices, however, can not be expelled because surrounded by superconducting material.

Once the best surface treatment is determined, in order to really maximize Q-factors when the cavity is horizontally placed inside the cryomodule, it is necessary to take into account all these results. In particular, it is crucial to screen very well the cryomodule from the external magnetic field, and especially from the orthogonal magnetic field component. Essential is also the optimization of the cavity cool-down in order to maximize the magnetic flux expulsion. 


\section{BIBLIOGRAPHY}

[1] A. B. Pippard, An experimental and theoretical study of the relation between magnetic field and current in a superconductor, Proc. R. Soc. Lond. A 216 (1953), p. 547

[2] J. Halbritter, FORTRAN-Program for the Computation of the Surface Impedance of Superconductors, KFK-Extern 3/70-6 (1970)

[3] B. Aune, R. Bandelmann, D. Bloess, B. Bonin, A. Bosotti, M. Champion, C. Crawford, G. Deppe, B. Dwersteg, D. Edwards, H. Edwards, M. Ferrario, M. Fouaidy, P. Gall, A. Gamp, A. Gössel, J. Graber, D. Hubert, M. Hüning, M. Juillard, T. Junquera, H. Kaiser, G. Kreps, M. Kuchnir, R. Lange, M. Leenen, M. Liepe, L. Lilje, A. Matheisen, W. Möller, A. Mosnier, H. Padamsee, C. Pagani, M. Pekeler, H. Peters, O. Peters, D. Proch, K. Rehlich, D. Reschke, H. Safa, T. Schilcher, P. Schmüser, J. Sekutowicz, S. Simrock, W. Singer, M. Tigner, D. Trines, K. Twarowski, G. Weichert, J. Weisend, J. Wojtkiewicz, S. Wolff and K. Zapfe, Superconducting TESLA cavities, Phys. Rev. ST Accel. Beams 3 (2000), p. 092001

[4] J. Knobloch, The $Q$ disease in superconducting niobium RF cavities, AIP Conference Proceedings 671 (2003) (1), p. 133

[5] A. Romanenko, A. Grassellino, A. C. Crawford, D. A. Sergatskov and O. Melnychuk, Ultra-high quality factors in superconducting niobium cavities in ambient magnetic fields up to $190 \mathrm{mG}$, Appl. Phys. Lett. 105 (2014) (23)

[6] M. Martinello, M. Checchin, A. Grassellino, A. C. Crawford, O. Melnychuk, A. Romanenko and D. A. Sergatskov, Magnetic flux studies in horizontally cooled elliptical superconducting cavities, J. Appl. Phys. 118 (2015), p. 044505

[7] A. Grassellino, A. Romanenko, S. Posen, Y. Trenikhina, O. Melnychuk, D. Sergatskov, M. Merio, M. Checchin and M. Martinello, $N$ doping: progress in development and understanding, in Proc. of the 17th International Conference on RF Superconductivity (Whistler, BC, Canada, 2015), MOBA06, pp. 48-54

[8] M. Martinello, A. Grassellino, M. Checchin, A. Romanenko, O. Melnychuk, D. A. Sergatskov, S. Posen and J. F. Zasadzinski, Effect of interstitial impurities on the field dependent microwave surface resistance of niobium, Appl. Phys. Lett. 109 (2016), p. 062601

[9] J. Bardeen and M. J. Stephen, Theory on the motion of vortices in superconductors, Phys. Rev. 140 (1965), p. A1197

[10] M. Checchin, A. Grassellino, M. Martinello, A. Romanenko and J. Zasadzinski, Submitted to Supercond. Sci. Technol.

[11] A. Gurevich and G. Ciovati, Effect of vortex hotspots on the radio-frequency surface resistance of superconductors, Phys. Rev. B 87 (2013), p. 054502

[12] ATLAS collaboration, Observation of a new particle in the search for the Standard Model Higgs boson with the ATLAS detector at the LHC, Physics Letters B 716 (2012) (1), p. 1 
[13] CMS collaboration, Observation of a new boson at a mass of 125 GeV with the CMS experiment at the LHC, Physics Letters B 716 (2012) (1), p. 30

[14] A. Seryi, Unifying physics of accelerators, lasers and plasma (CRC Press, 2015)

[15] A. Todd, Emerging industrial applications of LINACS, in Proc. of the 19th International Linear Accelerator Conference (Chicago, Illinois, USA, 1998), FR104

[16] U. Amaldi and A. Degiovanni, Proton and carbon LINACS for hadron therapy, in Proc. of the 27th International Linear Accelerator Conference (Geneva, Switzerland, 2014), FRIOB02

[17] M. Yoshioka, Review of accelerator-based Boron Neutron Capture Therapy Machines, in Proc. of the 7th International Particle Accelerator Conference (Busan, Korea, 2016), THXB01, p. 3171

[18] A. Krasnykh, J. Neilson and A. Yeremian, Concept of RF Linac for Intra-pulse Multi-energy Scan, in Proc. of the 7th International Particle Accelerator Conference (Busan, Korea, 2016), THOAB02, p. 3180

[19] A. Grassellino, A. Romanenko, D. A. Sergatskov, O. Melnychuk, Y. Trenikhina, A. C. Crawford, A. Rowe, M. Wong, T. Khabiboulline and F. Barkov, Nitrogen and argon doping of niobium for superconducting radio frequency cavities: a pathway to highly efficient accelerating structures, Supercond. Sci. Tech. 26 (2013) (10), p. 102001

[20] P. Bishop, M. Checchin, H. Conklin, A. Crawford, E. Daly, K. Davis, M. Drury, R. Eichhorn, J. Fischer, F. Furuta, G. M. Ge, D. Gonnella, A. Grassellino, C. Grimm, T. Gruber, D. Hall, A. Hocker, G. Hoffstaetter, J. Kaufman, G. Kulina, M. Liepe, J. Maniscalco, M. Martinello, O. Melnychuk, T. OConnel, J. Ozelis, A. D. Palczewski, P. Quigley, C. Reece, A. Romanenko, M. Ross, A. Rowe, D. Sabol, J. Sears, D. A. Sergatskov, W. Soyars, R. Stanek, V. Veshcherevich, R. Wang and G. Wu, LCLS-II SRF cavity processing protocol development and baseline cavity performance demonstration, in Proc. of the 17th International Conference on RF Superconductivity (Whistler, BC, Canada, 2015), MOPB033, pp. 159-163

[21] M. Martinello, M. Checchin, A. Grassellino, O. Melnychuk, S. Posen, A. Romanenko, D. A. Sergatskov and J. F. Zasadzinski, Trapped flux surface resistance analysis for different surface treatments, in Proc. of the 17th International Conference on RF Superconductivity (Whistler, BC, Canada, 2015), MOPB015, pp. $115-119$

[22] M. Martinello, M. Checchin, A. Grassellino, O. Melnychuk, S. Posen, A. Romanenko, D. A. Sergatskov and J. F. Zasadzinski, Tailoring surface impurity content to maximize Q-factors of superconducting resonators, in Proc. of the 7th International Particle Accelerator Conference (Busan, South Korea, 2016), WEPMR003, pp. 2258-2261

[23] D. Gonnella, J. Kaufman and M. Liepe, Impact of nitrogen doping of niobium superconducting cavities on the sensitivity of surface resistance to trapped magnetic flux, J. Appl. Phys 119 (2016), p. 073904

[24] M. Checchin and M. Martinello, Analytic solution of the electromagnetic eigenvalues problem in a cylindrical resonator (2016) 
[25] H. Padamsee, J. Knobloch and T. Hays, RF superconductivity for accelerators (Wiley-VCH Verlag GmbH and Co., KGaA, Weinheim, 1998)

[26] G. Ciovati, AC/RF Superconductivity, in Proc. of the CAS - CERN Accelerator School: Superconductivity for Accelerators (Erice, Italy, 2013), p. 19

[27] T. P. Wangler, RF linear accelerators (Wiley-VCH Verlag GmbH and Co., KGaA, Weinheim, 2008)

[28] H. Podlech, Superconducting versus normal conducting cavities, in Proc. of the CAS - CERN Accelerator School: Course on High Power Hadron Machines (Bilbao, Spain, 2011), p. 151

[29] C. Arnaud, P. Bernard, D. Bloess, G. Cavallari, E. Chiaveri, E. Ciapala, W. Erdt, G. Geschonke, I. Gorine, D. Güsewell, E. Haebel, H. Lengeler, G. Passardi, J. Schmid, R. Stierlin, J. Tückmantel and W. Weingarten, Status report on superconducting $\mathrm{Nb}$ cavities for LEP, in Proc. of the 4th Workshop on RF Superconductivity (KEK, Tsukuba, Japan, 1989), SRF89A02

[30] W. Meissner and R. Ochsenfeld, Ein neuer effekt bei eintritt der supraleitfhigkeit, Physica Naturwissenschaften 21 (1933), p. 787

[31] F. London and H. London, The Electromagnetic equations of the supraconductors, Proc. R. Soc. Lond. A 149 (1935), p. 71

[32] C. J. Gorter and H. Casimir, On supraconductivity I, Physica 1 (1934), p. 306

[33] M. Tinkham, Introduction to superconductivity (Dover Publications, 2004)

[34] G. E. H. Reuter and E. H. Sondheimer, The theory of the anomalous skin effect in metals, Proc. R. Soc. Lond. A 195 (1948) (1042), p. 336

[35] V. L. Ginzburg and L. D. Landau, Zh. Eksp. Teor. Fiz. 20 (1950), p. 1064

[36] R. P. Huebener, Magnetic flux structures in superconductors (Springer-Verlag, Berlin, Heidelberg, GmbH, 2001)

[37] J. Bardeen, L. N. Cooper and J. R. Schrieffer, Theory of superconductivity, Phys. Rev. 108 (1957), p. 1175

[38] W. S. Corak, B. B. Goodman, C. B. Satterthwaite and A. Wexler, Exponential temperature dependence of the electronic specific heat of superconducting vanadium, Phys. Rev. 96 (1954), p. 1442

[39] J. P. Turneaure, J. Halbritter and H. A. Schwettman, The surface impedance of superconductors and normal conductors: The Mattis- Bardeen theory, J. Supercond. 4 (1991) (5), p. 341

[40] D. C. Mattis and J. Bardeen, Theory of the anomalous skin effect in normal and superconducting metals, Phys. Rev. 111 (1958), p. 412

[41] R. E. Glover and M. Tinkham, Conductivity of superconducting films for photon energies between 0.3 and 40kTc, Phys. Rev. 108 (1957), p. 234

[42] A. Gurevich, Superconducting radio-frequency fundamentals for particle accelerators, Rev. Accl. Sci. Tech 05 (2012), p. 119 
[43] P. Flécher, Ph.D. thesis, University of Karlsruhe (1970)

[44] O. Melnychuk, A. Grassellino and A. Romanenko, Error analysis for intrinsic quality factor measurement in superconductingradio frequency resonators, Rev. Sci. Instrum. 85 (2014), p. 124705

[45] H. Padamsee, RF Superconductivity: Volume II: science, technology and applications (Wiley-VCH Verlag GmbH and Co., KGaA, Weinheim, 2009)

[46] M. Checchin, M. Martinello, A. Romanenko, A. Grassellino, D. A. Sergatskov, S. Posen, O. Melnychuk and J. F. Zasadzinski, Quench-induced degradation of the quality factor in superconducting resonators, Phys. Rev. Applied 5 (2016), p. 044019

[47] C. Antoine, How to achieve the best SRF performance: (practical) limitations and possible solutions, in Proc. of the CAS - CERN Accelerator School: Superconductivity for Accelerators (Erice, Italy, 2013), p. 209

[48] A. Grassellino et al., A pathway to SRF niobium cavities processing simplification and quality factor improvement via elimination of the post-annealing chemical treatment (2013)

[49] A. Romanenko, F. Barkov, L. D. Cooley and A. Grassellino, Proximity breakdown of hydrides in superconducting niobium cavities, Superconductor Science and Technology 26 (2013) (3), p. 035003

[50] G. Ciovati, Effect of low-temperature baking on the radio-frequency properties of niobium superconducting cavities for particle accelerators, J. Appl. Phys. 96 (2004) (3), pp. 1591-1600

[51] A. Romanenko, C. J. Edwardson, P. G. Coleman and P. J. Simpson, The effect of vacancies on the microwave surface resistance of niobium revealed by positron annihilation spectroscopy, Appl. Phys. Lett. 102 (2013) (23), 232601

[52] A. Romanenko, A. Grassellino, A. Barkov and J. P. Ozelis, Effect of mild baking on superconducting niobium cavities investigated by sequential nanoremoval, Phys. Rev. ST Accel. Beams 16 (2013), p. 012001

[53] Y. Trenikhina, A. Grassellino, O. Melnychuk and A. Romanenko, Characterization of nitrogen doping recipes for the Nb SRF cavities, in Proc. of the 17th International Conference on RF Superconductivity (Whistler, BC, Canada, 2015), MOPB055, p. 159

[54] A. Romanenko, A. Grassellino, O. Melnychuk and D. A. Sergatskov, Dependence of the residual surface resistance of superconducting radio frequency cavities on the cooling dynamics around Tc, J. Appl. Phys. 115 (2014), p. 184903

[55] B. W. J. McNeil and N. R. Thompson, X-ray free-electron lasers, Nat. Photon 4 (2010), pp. 814-821

[56] M. Waldrop, The big guns, Nature 505 (2014), p. 604

[57] C. Benvenuti, S. Calatroni, I. E. Campisi, P. Darriulat, M. A. Peck, R. Russo and A.-M. Valente, Study of the surface resistance of superconducting niobium films at $1.5 \mathrm{GHz}$, Physica C 316 (1999), p. 153 
[58] T. Kubo, Flux trapping in superconducting accelerating cavities during cooling down with a spatial temperature gradient, Prog. Theor. Exp. Phys. (2016), p. $053 \mathrm{G} 01$

[59] S. Posen, M. Checchin, A. C. Crawford, A. Grassellino, M. Martinello, O. S. Melnychuk, A. Romanenko, D. A. Sergatskov and Y. Trenikhina, Efficient expulsion of magnetic flux in superconducting radiofrequency cavities for high $Q_{0}$ applications, J. Appl. Phys 119 (2016), p. 213903

[60] S. Posen, M. Checchin, A. Crawford, A. Grassellino, M. Martinello, O. Melnychuk, A. Romanenko and D. Sergatskov, Flux expulsion variation in SRF cavities, in Proc. of the 17th International Conference on RF, Superconductivity (Whistler, BC, Canada, 2015), MOPB104, pp. 404-408

[61] M. Martinello, M. Checchin, A. Grassellino, O. Melnychuk, A. Romanenko, D. Sergatskov and J. Zasadzinski, Magnetic flux expulsion in horizontally cooled cavities, in Proc. of the 17th International Conference on RF Superconductivity (Whistler, BC, Canada, 2015), MOPB014, pp. 110-114

[62] L. H. Allen and J. H. Claassen, Technique for measuring the elementary pinning force in thin films, Phys. Rev. B 39 (1989), p. 2054

[63] J. Knobloch, H. Muller and H. Padamsee, Design of a high speed, high resolution thermometry system for $1.5 \mathrm{GHz}$ superconducting radio frequency cavities, Rev. Sci. Instrum. 65 (1994), p. 3521

[64] J. Halbritter, On surface resistance of superconductors, Z. Physik 266 (1974), pp. 209-217

[65] A. Gurevich, Reduction of dissipative nonlinear conductivity of superconductors by Static and Microwave Magnetic Fields, Phys. Rev. Lett. 113 (2014), p. 087001

[66] B. P. Xiao, C. E. Reece and M. J. Kelley, Superconducting surface impedance under radiofrequency field, Physica C 490 (2013), p. 26

[67] A. Romanenko, A. Grassellino, F. Barkov, A. Suter, Z. Salman and T. Prokscha, Strong Meissner screening change in superconducting radio frequency cavities due to mild baking, Appl. Phys. Lett. 104 (2014), p. 072601

[68] D. Gonnella, R. Eichhorn, F. Furuta, M. Ge, D. Hall, V. Ho, G. Hoffstaetter, M. Liepe, T. O'Connell, S. Posen, P. Quigley, J. Sears, V. Veshcherevich, A. Grassellino, A. Romanenko and D. A. Sergatskov, Nitrogen-doped 9-cell cavity performance in a test cryomodule for LCLS-II, J. Appl. Phys. 117 (2015), p. 023908

[69] J. C. Slater, Microwave Electronics, Rev. Mod. Phys. 18 (1946), p. 441

[70] E. Morenzoni, F. Kottmann, D. Maden, B. Matthias, M. Meyberg, T. Prokscha, T. Wutzke and U. Zimmermann, Generation of very slow polarized positive muons, Phys. Rev. Lett. 72 (1994), pp. 2793-2796

[71] C. Vallet, M. Bolor, B. Bonin, J. P. Charrier, B. Daillant, J. Gratadour, F. Koechlin and H. Safa, Flux trapping in superconducting cavities, in Proc. of the 3rd European Particle Accelerator Conference (Berlin, Germany, 1992), p. 1295 
[72] G. Ciovati and A. Gurevich, Measurement of RF losses due to trapped flux in a large-grain niobium cavity, in Proc. of the 13th International Workshop on RF Superconductivity (Beijing, China, 2007), TUP13, p. 132

[73] C. Caroli, P. G. de Gennes and J. Matricon, Bound fermion states on a vortex line in a type II superconductor, Phys. Lett. 9 (1964), p. 307

[74] J. I. Gittleman and B. Rosenblum, Radio-frequency resistance in the mixed state for subcritical currents, Phys. Rev. Lett. 16 (1966), p. 734

[75] Y. B. Kim, C. F. Hempstead and A. R. Strnad, Flux-flow resistance in type-II superconductors, Phys. Rev. 139 (1965), p. A1163

[76] R. Marcon, R. Fastampa, M. Giura and E. Silva, Vortex-motion dissipation in high-Tc superconductors at microwave frequencies, Phys. Rev. B 43 (1992), p. 2940

[77] E. H. Brandt, The flux-line lattice in superconductors, Rep. Prog. Phys. 58 (1995), p. 1465

[78] S. Casalbuoni, E. A. Knabbe, J. Kötzler, L. Lilje, L. von Sawilski, P. Schmüser and B. Steffen, Surface superconductivity in niobium for superconducting RF cavities, Nucl. Instr. Meth. Phys. Res. A 538 (2005), p. 45

[79] T. Kommers and J. Clarke, Measurement of microwave-enhanced energy gap in superconducting aluminum by tunneling, Phys. Rev. Lett. 38 (1977), pp. 10911094

[80] J. E. Mooij, Nonequilibrium superconductivity, phonons, and Kapitza boundaries (Springer US, Boston, MA, 1981), chap. Enhancement of superconductivity, pp. $191-229$

[81] G. M. Eliashberg, Film superconductivity stimulated by a high-frequency field, ZhETF Pis. Red. 11 (1970), p. 186

[82] A. Romanenko and A. Grassellino, Dependence of the microwave surface resistance of superconducting niobium on the magnitude of the rf field, Appl. Phys. Lett. 102 (2013), p. 252603 